Michaël Abecassis et

Gudrun Ledegen (éds)

\title{
De la genèse de la langue à Internet
}

Variations dans les formes, les modalités et les langues en contact 


\section{Modern French Identities}

Ce recueil d'articles regroupe une sélection des communications présentées au colloque international et pluridisciplinaire tenu à Oxford en janvier 2013, que complètent quelques contributions d'éminents chercheurs sur l'évolution du français, depuis ses origines jusqu'à ses développements liés à l'influence d'Internet. Les auteurs de ce volume s'intéressent à la langue française sous toutes ses formes et dans toutes ses représentations, dans le cinéma ou dans la littérature, et l'abordent aussi bien à travers sa syntaxe, son lexique, sa phonologie, que dans ses modalités orales ou écrites. De la rencontre de ces différents éclairages émerge un portrait de la langue française du XXIe siècle, telle qu'elle est étudiée actuellement, dans les recherches, dans ses modes d'écriture contemporains, sur les terrains plurilingues de différentes villes.

Michaël Abecassis est Maître de Conférences à l'Université d'Oxford (Christ Church, University College et Wadham College). Ses champs de recherche sont la linguistique appliquée, la sociolinguistique, la grammaire et le cinéma.

Gudrun Ledegen est Maître de Conférences à l'Université de Rennes 2. Ses recherches portent sur la sociolinguistique, la syntaxe du français parlé et les langues en contact (françaiscréole réunionnais-LSF). 
De la genèse de la langue à Internet 


\title{
$\mathbf{M}$ odern $\mathbf{F}$ rench Identities
}

Edited by Peter Collier

\author{
Volume 118
}

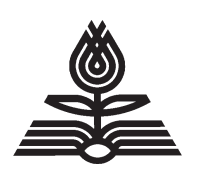

PETER LANG

Oxford • Bern • Berlin • Bruxelles $\cdot$ Frankfurt am Main $\cdot$ New York $\cdot$ Wien 


\section{Michaël Abecassis et \\ Gudrun Ledegen (éds)}

\section{De la genèse de la langue à Internet}

Variations dans les formes, les modalités et les langues en contact 
Bibliographic Information published by the Deutsche Nationalbibliothek

The Deutsche Nationalbibliothek lists this publication in the Deutsche Nationalbibliografie; detailed bibliographic data is available in the internet at http://dnb.d-nb.de.

A catalogue for this book is available from the British Library. Library of congress Control Number: 2014956438

ISSN 1422-9005 • ISBN 978-3-0343-1798-6 (Print)

E-ISBN 978-3-0353-0701-6 (E-PDF) • E-ISBN 978-3-0353-9783-3 (EPUB)

E-ISBN 978-3-0353-9782-6 (MOBI) • 10.3726/978-3-0353-0701-6

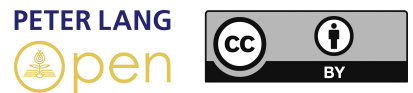

Open Access: This work is licensed under a Creative Commons Attribution

CC-BY 4.0 license. To view a copy of this license, visit https://creativecommons.org/licenses/by/4.0/

This publication has been peer reviewed.

๑) Michaël Abecassis, Gudrun Ledegen, 2015

Peter Lang AG

International Academic Publishers

www.peterlang.com 


\section{Table des matières}

MICHAËL ABECASSIS \& GUDRUN LEDEGEN

Introduction

I Confrontations d'oraux et d'écrits

CHANTAL LYCHE

Liaison et formation de mots : l'éclairage du français louisianais

JEANNE GONAC'H, GUDRUN LEDEGEN \& MARION BLONDEL

Ecrits en contexte de surdité : de la variation et de ses spécificités

ANNE-CAROLINE FIÉVET \& ALENA PODHORNÁ-POLICKÁ

L'appropriation territoriale par les jeunes à travers l'étude d'un

corpus de messages envoyés à la radio Skyrock

GUDRUN LEDEGEN \& CHANTAL LYCHE

La particule négative $n e$ dans les français d'Afrique

et de l'Océan Indien : convergences et divergences

II Le français : les mots et les structures

FRANÇOIS GAUDIN

Les (nouveaux) mystères de la variation lexicale 
vi

JEAN PRUVOST

D’une réflexion sur les dictionnaires électroniques

123

CAROLINE ROSSI

Les mots complexes en français contemporain :

pour qui n'a pas grandi avec le «Dinotrain »

BÉATRICE AKISSI BOUTIN \& NATHALIE ROSSI-GENSANE

Quelle(s) diversité(s) pour la syntaxe?

155

III Français et multilinguisme urbanisé

179

SOUHEILA HEDID

Le français dans le plurilinguisme urbain algérien :

les jeunes en parlent

ASMA CHAMLY-HALWANI

Le français autrement

$20 \mathrm{I}$

GEMMA KING

Decentring France: Multilingualism and the French language

in Philippe Lioret's Welcome (2009)

MARCELLINE BLOCK

The Message of the Text and the Text of the Message in Two

Contemporary French Films: LOL (Laughing Out Loud) and De Rouille et d'Os

Notices biographiques

Index 


\section{Introduction}

Ce recueil d'articles regroupe la plupart des recherches présentées au congrès pluridisciplinaire qui a eu lieu à Oxford en janvier 20I3, auxquelles s'ajoutent les contributions d'éminents chercheurs sur l'évolution du français de ses origines à nos jours avec le développement d'Internet. Les auteurs de ce volume s'intéressent à la langue française sous toutes ses formes et dans toutes ses représentations dans le cinéma ou dans la littérature. Ils traitent aussi bien du français à travers sa syntaxe, son lexique, sa phonologie, que dans ses modalités orales ou écrites ; c'est de la confrontation de ces différents éclairages qu'émerge une image de la langue française telle qu'elle est actuellement étudiée en recherches, travaillée en écritures, explorée dans le plurilinguisme urbain de divers terrains ...

Dans son article ouvrant le premier volet de cet ouvrage, qui pose centralement les pratiques orales et écrites, Chantal Lyche élargit la base empirique des études sur la liaison par la présentation et l'analyse de données tirées du français louisianais, une variété non écrite par les locuteurs qui n'ont qu'une pratique orale de la langue. Son analyse passe en revue la problématique et le rôle que la graphie peut jouer dans la liaison, avant d'étudier l'apport de quelques lettres écrites au $19^{\mathrm{e}}$ siècle par des locuteurs louisianais peu lettrés. Le corps de l'analyse consiste en une présentation du système louisianais contemporain, discuté à la lumière du créole louisianais et argumentant en faveur d'un traitement pluriel, non homogène de la liaison dans cette variété, combinant règle d'épenthèse, approche lexicale, analyse morphologique. Jeanne Gonac'h, Gudrun Ledegen et Marion Blondel présentent une recherche sur des écrits contrastés (copies d'examen d'université et SMS) produits par une scripteuse sourde. Cette confrontation de corpus avec les revues de la littérature révèle des traits spécifiques, dits « pi-sourds », à l'écrit produit par les personnes sourdes 
présents autant dans des écrits libres que fortement contraints. Par ailleurs, la littéracie universitaire révèlera ses effets particuliers, oscillant entre technolecte et insécurité linguistique. L'article d'Anne-Caroline Fiévet \& Alena Podhorná-Polická propose un regard sur les enjeux identitaires des jeunes lors de leur participation aux émissions de libre antenne à la radio. Un corpus de messages (SMS ou site internet de la station) envoyés par les auditeurs à la radio Skyrock est analysé, plus particulièrement sous l'angle de l'appropriation territoriale à travers les dédicaces et les pseudonymes à composante toponymique. Ce corpus datant de 2005 , elles questionnent également l'évolution de ces pratiques à l'heure de la convergence numérique et du débat autour de la réforme territoriale. L'article de Gudrun Ledegen et Chantal Lyche qui suit étudie la particule négative ne dans divers français d'Afrique et un français de l'Océan Indien, à savoir le français de La Réunion : en confrontant ces différents terrains africains et le terrain réunionnais par le biais des enquêtes du programme PFC (Phonologie du Français Contemporain) d'une part, et les pratiques innovantes des SMS d'autre part, il tente de cerner les convergences et divergences entre ces différentes variétés de français, et d'étudier les influences possibles du contact linguistique. Les analyses révèlent un maintien élevé pour le terrain africain versus un alignement sur le système du français de référence pour le terrain réunionnais : la vernacularisation, le contact de langue, la prosodie et la phonologie, les modalités de l'oral face à l'écrit, se révèlent des facteurs explicatifs qui viennent, ensemble, éclairer les variations syntaxiques.

« Le français : les mots et les structures » est l'objet du deuxième volet. La notion de variation lexicale est devenue d'un usage fréquent. François Gaudin examine sa compatibilité avec la notion d'invariant d'un point de vue linguistique, sociolinguistique et historique, en s'appuyant sur la tradition lexicographique de description du français. Il interroge également son caractère heuristique en croisant des exemples diversifiés, en posant le regard de sociolinguiste au centre de sa problématique. Partir à la recherche des mots de la langue française, comme le fait Jean Pruvost, en reconstituer l'histoire connue en examinant les dictionnaires disponibles, ceux encore irremplaçables sur papier, et ceux offert sur Internet ou sur cédérom, voilà un parcours qui ne va pas sans l'examen des possibilités offertes. Les bonnes et les mauvaises surprises y sont légions, d'où l'intérêt d'une bonne 
connaissance des différents sentiers qui parcourent la forêt des dictionnaires pour pouvoir organiser la meilleure traque possible. Longtemps négligés ou peu décrits autrement que comme « mots composés », catégorie à géométrie variable puisque pour certains linguistes au moins elle comprend aussi bien des noms modifiés par un adjectif ou par un syntagme prépositionnel, les mots complexes comme « fleur-horloge » ou « Dinotrain » traités par Caroline Rossi font l'objet d'un regain d'intérêt. Des travaux récents ont démontré leur productivité en français contemporain. Ils nous intéressent ici en tant que créations lexicales permettant de véhiculer de nouvelles significations. Elle présente et discute les résultats d'une expérience pilote en classe de $\mathrm{CE}_{2}-\mathrm{CM}_{\mathrm{r}}$, qui montre une préférence assez nette des locuteurs pour des mots complexes lorsqu'il s'agit de nommer des objets hybrides. L'article de Béatrice Akissi Boutin et Nathalie Rossi-Gensane souhaite attirer l'attention sur l'insuffisance des notions de variables et de variantes, à la faveur de certains faits souvent rangés dans la variation syntaxique, qui sont ici observés sur l'ensemble des espaces de la francophonie : les interrogatives totales (directes), la présence ou absence du subjonctif, l'alternance entre conditionnel « classique » et « conditionnel périphrastique » (aller à l'imparfait suivi de l'infinitif), certaines réalisations indéterminées des auxiliaires avoir et être et, enfin, les structures s'organisant autour de la présence $v s$ absence de que. Il est notamment souligné que le traitement des diversités syntaxiques se heurte à une double intrication : celle de la syntaxe avec les autres niveaux linguistiques (phonique, prosodique, sémantique, pragmatique, discursif) et celle des facteurs extralinguistiques entre eux, notamment diatopiques et diaphasiques.

Dans ce dernier volet consacré au français et multilinguisme urbanisé, Souheila Hedid met l'accent sur le plurilinguisme urbain tel qu' il est vécu et perçu par les jeunes. Les données exploitées ici résultent d'une enquête de terrain menée auprès des jeunes algériens urbains. L'objectif était d'adopter une nouvelle approche méthodologique pour appréhender leurs pratiques langagières et leurs représentations sociolinguistiques vis-à-vis des langues de/dans leur ville. Asma Chamly-Halwani met en relief le rapport qu'entretiennent les écrivains libanais avec la langue française. Grâce à leur capacité d'enrichissement expressif, combinant arabe et français, Maalouf, dans Les Identités meurtrières perçoit la langue française comme sa langue de cœur ; 
la figure féminine d'Eddé, dans Cerf-volant, est surtout attirée par ce dialogue permanent lequel sétablit entre ces deux langues de culture, l'arabe et le français, déterminant pour elle deux représentations du monde d'autant plus enrichissantes qu'elles s'avèrent différenciées. Quant à Stétié, le français se révèle pour lui la Parole dans laquelle s'effectue sa quête ontologique. Le cinéma offre des ressources inestimables pour l'analyse linguistique et diachronique. Ecrits pour être joués les scénarios sont à cheval entre l'écrit et l'oral. Ils véhiculent non seulement des variétés linguistiques diastratiques et diaphasiques de l'oralité, mais figent des tours écrits à travers des enseignes, des affiches qui apparaissent dans l'arrière-plan ou autres objets diégétiques, tout comme par le biais de lettres ou de SMS mis en abyme dans la tessiture du film. Dans un grand nombre de films français contemporains, à l'instar de la littérature, le multilinguisme s'affirme comme un élément narratif important, comme nous le montre Gemma King. Dans ces films, les langues jouent un rôle essentiel dans le récit et la caractérisation des personnages; en particulier, les langues représentent très souvent un outil de pouvoir. L'un des films les plus proéminents du cinéma français multilingue est Welcome (Philippe Lioret, 2009), un film qui comprend des passages en français, anglais, kurde et pashto. Par contraste avec le cinéma français traditionnel traitant de l'immigration, dans Welcome, la mise en scène de l'espace périphérique de Calais et la focalisation sur un protagoniste kurde clandestin qui cherche à émigrer à Londres sert à « décentrer »le rôle de la France, et par conséquent le rôle du français. Dans ce contexte globalisé et multilingue, où l'anglais a plus de portée que le français, la domination de la langue française est mise en question. Marcelline Block examine enfin la signification du SMS dans des films récents : la comédie romantique LOL (Laughing Out Loud) ${ }^{\circledR}$ (2009) réalisé par Lisa Azuelos et le drame De rouille et d'os (Jacques Audiard, 20I2). L'auteure considère la façon dont ces deux films traitent et représentent le SMS aux niveaux du langage et du vocabulaire du « texto » (surtout les acronymes comme « LOL » inscrit dans le titre du film $L O L$, et les néologismes comme « opé » dans De rouille et d'os), de la culture, et de la signification.

Ainsi, comme l'indique le sous-titre de notre ouvrage, la variation est un fil rouge qui traverse les différentes contributions : observée en partant des productions pour ce qui est de la phonologie (Lyche), de la 
syntaxe (Gonac'h, Ledegen \& Blondel ; Lyche \& Ledegen ; Boutin \& Rossi-Gensane), du lexique (Gaudin ; Pruvost ; Rossi), voire de la variation diachronique du lexique cernée à travers les dictionnaires (Pruvost). Enfin, la variation est plus centralement interrogée en tant que notion applicable (ou non) au domaine lexical (Gaudin) ou syntaxique (Boutin \& RossiGensane), et devant cette problématique, des traitements pluriels sont proposés (Lyche ; Gonac'h, Ledegen \& Blondel). Les modalités orales et écrites forment un autre canevas sur lequel se fixent les différents textes : pour l'oral, la problématique de la liaison (Lyche), pour la confrontation des deux modalités, celle des SMS (Gonac'h, Ledegen \& Blondel, Fiévet \& Podhorná-Polická) ou la comparaison entre enquêtes phonologiques et SMS (Ledegen \& Lyche). Par ailleurs, l' « idéologie du standard » (Milroy \& Milroy 1989), particulièrement vivante pour le français, se construit aussi à la lumière du contraste entre ces modalités, que ce soit pour les mots (Gaudin ; Rossi) ou pour les structures (Boutin \& Rossi-Gensane). L'histoire de la langue (Pruvost), tout comme la littérature (ChamlyHalwani) et le cinéma (King; Block) relèvent plus spécifiquement de la modalité écrite, tout en intégrant l'oralité dans ses attestations ou créations. Enfin, le multilinguisme est considéré à partir des discours épilinguistiques de jeunes locuteurs algériens (Hedid) ou d'écrivains libanais (ChamlyHalwani), et dans le cinéma contemporain (King; Block), mais il est aussi présent dans les contributions des autres parties, en tant que réalité sociolinguistique incontournable dans les analyses de pratiques (Gonac'h, Ledegen \& Blondel; Ledegen \& Lyche; Gaudin). 

Confrontations d'oraux et d'écrits 



\section{Liaison et formation de mots : l'éclairage du français louisianais}

La liaison en français reste l'un des phénomènes de sandhi les plus étudiés dans le cadre de la phonologie générative (et post générative) depuis la fin des années 1960 marquées par la parution en 1968 de The Sound Pattern of English (Chomsly et Halle 1968) et de l'ouvrage de Schane sur la phonologie du français (Schane 1968) ${ }^{1}$. La base empirique de la plupart des travaux sur la liaison est longtemps provenue de données prescriptives (Fouché 1959), mais elle s'est vue considérablement renouvelée dans l'ouvrage majeur d'Encrevé (1988), puis par la suite grâce aux données issues de corpus, géographiquement limités (De Jong 1994) ou non (Durand et Lyche 2008, Mallet 2008, Côté 2005, 2010, 2013). Des données acquisitionnelles (Chevrot et al. 2009, Wauquier et Braud 2005), psycholinguistiques (Spinelli et al. 2003), phonétiques, ont également contribué à l'enrichissement de cette base empirique (voir Soum et Coquillon 2014) et nous autorisent à mieux cerner le phénomène dans sa globalité. En dépit de cette riche masse de données, certaines questions fondamentales concernant la liaison n'ont pas reçu de réponse tranchée, et en particulier la littérature n'offre aucun consensus sur le statut lexical de la consonne de liaison. Nous nous proposons dans ce chapitre d'élargir quelque peu la base empirique des études sur la liaison par la présentation et l'analyse de données tirées du français louisianais, une variété non écrite par les locuteurs qui n'ont qu'une pratique orale de la langue. Après un bref rappel de la problématique et du rôle que la graphie peut jouer dans la liaison (Section I), nous étudierons l'apport de quelques lettres écrites au $19^{\mathrm{e}}$ siècle par des locuteurs louisianais peu lettrés (Section 2)

I Ce travail a bénéficié des commentaires bienveillants de Jacques Durand que je tiens à remercier. 
avant de présenter le système louisianais contemporain (Section 3). Nous discuterons les données à la lumière du créole et argumenterons en faveur d'un traitement non homogène de la liaison dans cette variété (Section 4) avant de conclure (Section 5 ).

\section{Liaison et illettrisme : la problématique}

Rappelons que la liaison est un phénomène de sandhi par lequel une consonne se voit réalisée sous certaines conditions, linguistiques et sociolinguistiques. Ce sont les premières qui constituent l'objet principal de cette étude. Ainsi, dans une suite Mot-I + Mot-2, une consonne de liaison peut être associée au Mot-2 (liaison dite enchaînée) lorsque ce dernier est à initiale vocalique : les [z] années avec liaison /z/ s'oppose à les /mois sans liaison possible. Les consonnes susceptibles de faire liaison sont réduites à un petit nombre : $/ \mathrm{n}, \mathrm{z}, \mathrm{t}, \mathrm{b}, \mathrm{p} / \mathrm{+} / \mathrm{l} /$ dans les variétés nord-américaines ${ }^{2}$. La consonne est normalement perçue comme l'attaque du Mot-2, créant parfois des suites homophones, comme dans l'exemple (I) maintes fois cité dans la littérature.

(I) [pœtitami] petit ami petite amie petit tamis

Un ensemble d'études phonétiques s'accordent néanmoins sur l'absence d'homophonie complète entre consonne de liaison, consonne enchaînée et consonne initiale fixe (par ex. Delattre 1966, Dejean de la Bâtie 1993). De plus, des tests perceptifs font état d'une détection moins rapide de la consonne de liaison (Nguyen et al. 2007) que de la consonne initiale. Ces différences acoustiques et perceptives interviennent régulièrement dans 
le débat théorique portant sur le statut lexical de la consonne de liaison sous la forme d'arguments contre la présence dans la représentation, de la consonne de liaison à l'initiale du Mot- $2^{3}$.

La question toujours non résolue du statut lexical de la consonne de liaison perdure depuis les années 70 lorsque Klausenburger (1974) rejette l'analyse par troncation en vigueur (Schane 1968, Selkirk 1972) pour proposer une analyse par épenthèse. Dans le premier cas, la consonne de liaison est associée au Mot-I dans sa représentation, dans le second, elle est insérée par règle dans certains contextes définis. Une troisième thèse, celle de la supplétion, intègre la consonne au Mot-I, entraînant une allomorphie généralisée (nous /nu ; nuz/, soit au Mot-2 sous forme de préfixe (ami /ami ; z+ami/ (Morin 2003) ou non /ami ; zami ; nami ; tami/ (Ternes 1977). Si l'analyse par troncation du type Schane (1968) a été généralement abandonnée, l'association de la consonne au Mot-I subsiste sous une nouvelle forme largement développée dans le cadre de la phonologie multilinéaire : la consonne de liaison a bien son origine dans le Mot-I, mais elle n'a pas d'ancrage dans le squelette, elle est flottante. Non réalisée (et non plus tronquée) dans certaines conditions phonologiques, elle sera associée au squelette si l'attaque suivante est vide, ce qui induira sa réalisation (Encrevé 1988, Wauquier-Gravelines et Braud 2005). Dans ce dernier scénario, les auteurs font prévaloir un phénomène de « bootstrapping » morphologique par lequel la morphologie aide les structures phonologiques à se mettre en place : l'enfant, dès l'âge de 3 ans, tire profit de ses observations sur la morphologie d'un ensemble de mots (petit, petite, petitesse) pour encoder dans les représentations une consonne flottante (petit / pati(t)/). Il s'agit ici d'une approche exclusivement phonologique et uniforme de la liaison qu'elle soit catégorique ou non, pré- ou postposée à une catégorie principale.

Il nous est impossible dans le cadre de ce travail de citer ne serait-ce qu'une infime partie des travaux sur la liaison. Nous renvoyons donc le lecteur à Encrevé (1988), Côté (20II) et aux différents chapitres de Soum et Coquillon (2014) pour une bibliographie plus complète. 
La nature des données venues étayer tel ou tel traitement contribue largement au débat théorique. Churma (1977), par exemple, rejette les arguments avancés par Klausenburger en faveur de l'épenthèse en faisant valoir que les données avancées ne sont pas conclusives. Il suggère d'interroger directement des locuteurs de préférence illettrés pour éliminer le biais orthographique ${ }^{4}$. Nous ne nous étendrons pas sur la difficulté à élaborer des tests fiables sur les intuitions des locuteurs, mais soulignerons que, comme le rappelle Encrevé (1988), langue écrite et langue parlée interagissent depuis des siècles, vérité indéniable dans le cas du français où la pression de la norme n'a jamais lâché prise. De nos jours, l'illettré francophone aura probablement appris sa langue dans un milieu lettré et il sera entouré de lettrés avec qui il interagit quotidiennement ${ }^{5}$. Si le rôle joué par la graphie dans les représentations reste à démontrer, des études récentes ont su mettre en valeur des différences importantes entre les registres dans l'usage de la liaison (inter alia Mallet 2008, Durand et Lyche 2008), et ce faisant l'impact de la graphie (Laks 2005). La comparaison des registres fournit en effet des résultats sans appel. Dans le corpus PFC (Durand, Laks et Lyche 2009), on observe 62,15\% de liaisons réalisées après est dans le texte lu alors que la conversation libre n'en fournit que $28,4 \%{ }^{6}$. Il suffit par ailleurs d'écouter la radio ou la télévision pour entendre de nombreuses liaisons probablement déclenchées, dans bien des cas, par la présence visuelle du mot écrit ${ }^{7}$. Laks (2005) oppose les liaisons

«A possible solution in this case would be simply to ask speakers what is 'stored in their heads' (i.e., ask for their intuitions about the underlying form of, say, petit). In order to guard against the possible influence of the orthography, it would presumably be necessary to use illiterates. » (Churma $1978:$ 149).

« L'illettré francophone métropolitain natif représente d'autant moins, aujourd'hui, la forme « pure » d'une langue qui s'écrit depuis plus de dix siècles, qu'il tient nécessairement cette langue d'un entourage de locuteurs au moins partiellement scolarisé ne serait-ce que cette forme d'entourage linguistique que représentent la radio et la télévision qui sont, on le sait, une des principales sources d'acquisition du français par les locuteurs immigrés. » (Encrevé 1988 : 134).

6 Consultation de la base le 6 juin 2013. <http://www.projet-pfc.net>

7 Citons par exemple sur la radio France Inter $(20.06 .2013,8 \mathrm{~h} 25)$ « les yeux grands $[\mathrm{z}] \mathrm{ou} / \mathrm{-}$ le journaliste hésite, son collègue le rassure par un 'oui' d'approbation grands $[\mathrm{z}]$ ouverts ». 
catégoriques préposées à une catégorie grammaticale aux liaisons variables, dans l'ensemble postposées à une catégorie grammaticale, qui font intervenir directement les connaissances de la graphie et qui seules constituent un lieu de variation fluctuant selon l'âge ou le milieu social. Il propose la création dans les représentations d'une ligne autosegmentale graphique (GRAPH) qui devrait être liée à la ligne autosegmentale $\mathrm{PHON}$ pour la réalisation de ces liaisons. Liaison catégoriques et liaisons variables ne seraient donc pas soumises au même traitement, un principe défendu également inter alia par Côté (2005), Durand et Lyche (2008).

Qu'en est-il de la ligne GRAPH chez des locuteurs qui n'ont aucune maîtrise de la graphie ? Doit-on envisager qu'en l'absence d'une telle ligne, ces locuteurs ne produisent pas de liaisons variables ou faut-il argumenter plutôt, comme le fait Encrevé, que tout locuteur de français subit d'une façon ou d'une autre, ne serait-ce que par son environnement, l'influence de la graphie?

Nous nous proposons de tenter de répondre à cette question en examinant de plus près le français louisianais, une variété qui perdure exclusivement oralement ${ }^{8}$, sans perdre de vue le débat théorique sur le statut lexical de la consonne de liaison.

\section{Le français louisianais : regard sur l'écrit}

La Hill Memorial Library de l'Université de l'Etat de Louisiane à Bâton Rouge (LSU) conserve de grandes collections de manuscrits écrits en français depuis la fondation de l'Etat. Parmi ces collections, ont été numérisées plus de $\mathrm{I} 40$ lettres écrites aux $\mathrm{I} 8^{\mathrm{e}}$ et $\mathrm{I}^{\mathrm{e}}$ siècles. Au-delà de leur valeur de mémoire, ces lettres nous donnent accès à l'écriture de certains locuteurs paysage sociolinguistique très diversifié. Voir Valdman (2007) pour une mise au point. 
peu scolarisés. Nous nous concentrerons ici sur quelques lettres écrites pendant la période qualifiée de «bilinguisme collectif » (Dubois 2010) qui s'étend de 1830 à I 870 et au cours de laquelle la Louisiane voit une immigration massive d'Américains et d'Européens, et en particulier d'Irlandais. Dans les petites communautés isolées, les immigrants apprennent la langue de la communauté (le plus souvent une variété de français louisianais) et les mariages accentuent le bilinguisme. Il s'agit souvent de locuteurs peu éduqués et dans ce sens, les locuteurs que nous avons sélectionnés sont typiques de cette population.

(2) Le corpus de lettres I828, lettre de Jean Bapt Pistache I845, lettre de la veuve Dicharry I863, récit de Thibodaux I860, Meullion family papers (Créoles de couleur)

Si les auteurs éprouvent quelques difficultés à respecter les accords, ils ont dans l'ensemble une excellente maîtrise des consonnes finales. On y observe néanmoins des simplifications du groupe obstruante + liquide (de si rende chez nous, « de s'y rendre chez nous » Thibodaux), simplification systématique dans la lettre de Pistache, pastiche du parler acadien de l'époque. Un $\langle\mathrm{e}\rangle$ final peut se voir insérer après un $\langle\mathrm{r}\rangle$, une erreur probablement due à la généralisation de la règle graphique selon laquelle un $\langle\mathrm{e}\rangle$ final conditionne la prononciation d'une consonne finale (le meilleure diner que nous avons eu, Thibodaux; pour que je puise partire, Meuillon).

Par rapport à la consonne de liaison, deux lettres font état d'erreurs systématiques, voulues dans la lettre de Pistache, mais probablement pas dans celle de la veuve Dicharry. Dans la lettre de Pistache, la consonne de liaison se trouve toujours graphiquement présente à l'initiale du Mot-2 pour indiquer le nombre dans la flexion verbale.

(3) La liaison comme marqueur du pluriel pour des places qui zappelons candidat; y faut qui zont la poche bin grasse ; c'est qu'zens profitons tout comme les autres ; 
et zallons toujours not train ;

vous zallez

L'agglutination graphique de la consonne de liaison au Mot-2 s'observe également dans les deux autres lettres, mais dans des cas isolés uniquement. En (3) en revanche, il s'agit d'une erreur volontaire pour souligner le pluriel verbal et non nominal puisque Pistache n'insère pas un $\langle z>$ à l'initiale de autres. Avec la veuve Dicharry, nous avons une lettre d'un style formel : cette femme écrit à un prêtre pour solliciter son aide dans une affaire privée. La lettre témoigne de la part du scripteur d'une très bonne maîtrise des consonnes finales (monsieur; boucoup) mais pas du déterminant (dé nouvelle alterne avec des nouvelles). La particularité de la lettre réside dans l'intégration systématique de la consonne de liaison dans tout Mot-2 à initiale vocalique (Lyche 2010) :

(4) Liaison dans le Mot-2 je vous zecri cette lettre ; vous zét si charitable; mé zamitié ; je vous zassure ; nous zavons ; mon nécriture

Ces données écrites, interprétées dans le cadre de la nature lexicale de la consonne de liaison, viennent valider une approche qui rattache la consonne de liaison à l'attaque du Mot-2, approche largement retenue pour décrire les premières années de l'apprentissage langagier (Chevrot, Dugua et Fayol 2009, Wauquier-Gravelines et Braud 2005).

Les erreurs des scripteurs peu lettrés évoquent l'écrit des enfants en apprentissage scolaire sans pour autant s'y conformer. En effet, les erreurs observées chez les enfants sont de nature beaucoup plus variée, comme l'illustre largement Soum (1997): une taction (une action); un grot source (un gros ours), formes totalement absentes dans notre corpus. Dans les lettres de Louisiane, tous les problèmes rencontrés sont liés uniquement au découpage de la chaîne. Nous nous tournons à présent vers le système louisianais contemporain chez des locuteurs qui ne pratiquent pas l'écrit en français. 


\section{Le français louisianais : système contemporain}

Le français louisianais constitue une source précieuse pour l'étude de la liaison du fait de l'absence de connaissances graphiques des locuteurs. L'enseignement est devenu obligatoire en Louisiane en 1916, mais il s'est toujours agi d'un enseignement en anglais à une population qui à l'époque était monolingue en français. Nos locuteurs francophones sont tous bilingues, ils ont suivi un cursus scolaire normal, mais ils n'ont aucune pratique de la lecture ou de l'écriture du français. Les données recueillies proviennent du corpus PFC et ont été rassemblées selon le protocole du programme (www.projet-pfc.net) (Durand et Lyche 2003, Durand, Laks et Lyche 2009) avec une accommodation nécessaire pour le terrain louisianais (Durand et Lyche 20I3, Klingler et Lyche 20I2) où les tâches de lecture ne pouvaient être entreprises.

$\mathrm{Si}$, a priori, rien ne semble distinguer le système de la liaison dans le français louisianais du système hexagonal (Papen et Rottet 1997), un regard plus approfondi met en exergue une quasi absence de liaisons variables et un système restreint de liaisons catégoriques (Klingler et Lyche 20I2). Les liaisons catégoriques sont confinées aux suites $\mathrm{PRO}_{\text {sujet }}+$ Verbe, DET + Substantif.

(5) Liaisons catégoriques Ils/eussses [z] arrivent Les, mes $[z]$ amis Un $[\mathrm{n}] \mathrm{ami}$

Les liaisons variables sont rares mais pas inexistantes. La liaison est très présente après la préposition en (en [n]hiver), sans pour autant être systématique; elle est moins fréquente après l'auxiliaire est (il est /arrivé vs il est [t]arrivé). La liaison ne s'étend néanmoins pas au verbe copule (il est/anglais, *il est [t]anglais). La forme progressive est après comme dans il est après acheter est lexicalisée et se réalise toujours [ztape]. Le nombre de liaisons variables se trouve encore contraint par la morphologie du français louisianais qui tend à remplacer l'auxiliaire être par avoir au passé composé (il a resté et non pas il est resté). Au-delà de ces quelques cas, la 
liaison variable est inexistante. En particulier les contextes PREP + DET (chez un malade) et ADJ + Substantif (petit homme) n'entraînent pas la liaison à moins que l'adjectif ne soit au pluriel petits $[z]$ hommes).

La liaison en /z/ dans cette variété est liée au marquage morphologique du pluriel : un gros /homme mais des gros [z]yeux. Cette particularité a été décrite par Aub-Büscher (1962) pour un dialecte parlé dans l'est de la France (Ranrupt) où la liaison entre l'adjectif et le substantif ne se réalise que dans un contexte pluriel. On notera en particulier la présence d'un /z/ pluriel avec les chiffres (cinq [z]enfants, cinq [z] heures de temps mais cinq / heures Klingler et Lyche 2012). Gougenheim (1938) voyait déjà dans la liaison en $/ \mathrm{z} /$ une marque morphologique, et le français louisianais témoigne de la force de cette marque?. La liaison pluriel ne s'étend néanmoins pas à l'adjectif postposé au nom (des puits /artésiens), mais elle peut apparaître dans une construction à sens pluriel sans être conditionnée par un Mot-I comme dans les exemples en (6).

(6) Liaison pluriel autonome

Eusses mettaient les patates dedans les, comme les, des sacs d' $[z]$ huîtres - Roland C'était des petits bateaux, euh, les bateaux à $[z]$ huîtres - Audrey

Il était en enfance pour, assez d'[z]années - Audrey

C'était des jeunes /hommes vs. Il y avait pas assez d'[z]hommes (Arnaudville, 04.20 .2013 )

Tous ces exemples vont dans le sens d'un pluriel marqué par une consonne de liaison insérée dans l'attaque vide d'un substantif au sein d'une unité prosodique étroitement liée. Notons en passant que l'on ne saurait argumenter en faveur d'une contrainte NO-HIATUS en français louisianais puisque l'enchaînement à lui seul aurait suffi à respecter cette contrainte (a-ssez-d'a-nnées). Le hiatus maintenu dans des puits lartésiens, ne constitue pas une violation de contrainte, mais bien une illustration du poids $\mathrm{du}$ mot lexical qui à lui seul constitue une unité prosodique porteuse d'accent (Lyche 2010).

9 Voir également le substantif euros régulièrement réalisé [z]euros après un chiffre (vingt [z]euros) en français hexagonal. 
Une seule variété de français louisianais fait apparemment exception à cette généralisation, le français de la paroisse Lafourche où les locuteurs alternent indifféremment entre les /affaires et les [z] affaires ${ }^{10}$ contexte habituellement catégorique à l'intérieur duquel les éléments sont fortement soudés prosodiquement et appartiennent à un mot prosodique unique. La paroisse Lafourche exhibe une alternance / $\mathrm{z}, \mathrm{h}, \varnothing /$ aussi bien à l'intérieur d'un mot qu'en liaison. Le substantif maison peut ainsi être réalisé [mezõ, mehõ, meõ], selon le locuteur. De la même façon, chez certains locuteurs,

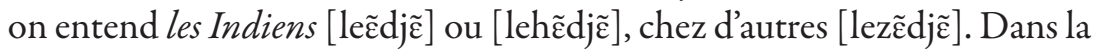
grammaire individuelle du locuteur, la variante qui prévaut dépend entièrement du substantif, certains substantifs conditionnent la liaison, d'autres pas ou alternent. Nous donnons en (7) quelques exemples pour le locuteur Roland issu du corpus Dubois.

(7) Conditionnement lexical de la liaison

Avec liaison

hommes, heures, huittres, arbres, ordres, ans, oiseaux

Sans liaison

indiens, habitations, abcès, ouvriers, orteils, acheteurs, étrangers

Alternance

affaires, animaux, enfants

Ces données ne sont pas compatibles avec une analyse selon laquelle la consonne de liaison trouve son origine dans le Mot-I et elles témoignent également d'une profonde similitude entre la consonne de liaison et toute autre fricative. Nous avons en effet évoqué dans la Section i le fait que la consonne de liaison se distinguerait acoustiquement d'une consonne initiale (De Jean de la Bâtie 1993), ce qui induirait une distinction phonologique. Ici, le comportement de la consonne à lui seul nous entraîne vers un traitement uniforme des fricatives alvéolaires ${ }^{11}$.

Les données de la paroisse Lafourche sont tirées du corpus de Sylvie Dubois (Dubois 2003).

II Il se peut qu'il s'agisse d'un changement en cours. Blainey (2013) n'observe pas ce phénomène dans un corpus de 1977. 
Nous avons vu dans la Section 2 que les scripteurs peu lettrés procèdent parfois à un découpage fautif de la chaîne écrite qui reflète probablement une incertitude quant à l'appartenance lexicale de la consonne de liaison. La même situation prévaut dans le français louisianais où une segmentation erronée donne lieu à une série de restructurations. Le dictionnaire du français louisianais (Valdman et al. 2010) inclut 29 entrées en / z/ alternant avec une entrée à initiale vocalique (agrafe/zagrafe, haricot/zaricot, oreille) zoreille, etc.). Les variantes à initiale consonantique ne sont pas limitées à une position post déterminant, comme en témoigne l'interjection zenfants (Zenfants! quoi je vas faire) qui a pris le sens de « mon Dieu ». Au-delà des entrées en $/ \mathrm{z} /$, le dictionnaire affiche deux entrées en $/ \mathrm{n} /$, toutes deux présentes dans notre corpus : oncle/noncle, anglais/nanglais (voir dans les entretiens avec Audrey : des fois Mom connaissait autant le français que le nanglais).

Le français louisianais fait état d'autres restructurations qui n'impliquent pas la consonne de liaison mais qui témoignent de l'ampleur du processus de segmentation fautive chez une population qui n'a pas accès à l'écrit. Certains substantifs affichent deux ou plusieurs entrées dans le dictionnaire de Valdman et al. (2010), certaines étant le fruit d'une segmentation incorrecte par rapport au français de référence.

(8) Segmentation et restructuration

Nombril-ombril

Numéro-luméro

Ombre-lombre-alombre

Elastique-alastique-lastique-astique

Américain-amaricain-méricain

Le dictionnaire illustre par exemple alombre à l'aide de la phrase suivante : $Y$ a pas d'alombre dessus ce bord-là de la maison. Ces cinq entrées trouvent aisément toutes leur explication dans des problèmes de segmentation : dans les deux premiers cas, la consonne nasale initiale est conçue comme une consonne de liaison, dans les trois derniers cas, c'est le déterminant défini qui entraîne différentes segmentations de la chaîne. Les deux derniers substantifs, élastique-américain, méritent une mention particulière dans la mesure où ils existent également comme adjectifs, mais sans la même 
allomorphie. En effet, comme adjectifs élastique alterne avec alastique, et américain avec amaricain, mais les formes tronquées (alastique, astique, méricain) sont exclusivement des substantifs. Nous pouvons donc conclure que seule la construction DET + SUBSTANTIF engendre des erreurs de segmentation qui mènent à l'allomorphie. L'allomorphie observée dans le français louisianais provient sans nul doute d'une segmentation fautive et généralisée de la chaîne sans que les suites avec liaison soient particulièrement ciblées. La proximité du créole louisianais qui connaît une restructuration massive pour ne maintenir uniquement qu'une structure de mots CVCV pourrait avoir influencé le développement du français louisianais. L'hypothèse nous semble a priori légitime puisque nous adoptons ici le point de vue selon lequel les créoles français sont « des systèmes linguistiques autonomes issus [...] de l'accélération et de la radicalisation de processus évolutifs qu'on peut observer, sous des formes et à des degrés différents, dans les français populaires ou marginaux $\gg(\text { Chaudenson } 1989: 84)^{12}$. Il n'en reste pas moins vrai que seul un nombre limité de restructurations a été mis en place en français louisianais et que la langue maintient un grand nombre de structures VCV ... ${ }^{13}$

\section{Liaison et restructuration lexicale}

Comme tous les autres créoles français, le créole louisianais affiche une structure de base CVCV (Klingler 2003, Neumann 1985) avec un lexique qui peut être divisé en formes agglutinées et formes non-agglutinées. Les formes agglutinées peuvent comprendre soit l'insertion d'une consonne d'attaque afin de respecter la structure CV obligatoire, soit l'insertion

I2 Voir aussi Bollée et Neumann-Holzschuh (1998).

I3 La fréquence de certaines constructions pourrait être à l'origine des restructurations. Cela semble évident pour zyeux mais beaucoup moins pour lastique. 
d'une syllabe complète. Les exemples en (9) sont tirés de Klingler (2003) et Neumann (1985).

(9) Agglutination en créole louisianais

a) Insertion d'une consonne d'attaque

Mo lasyet « mon assiette »

En zépron « un épron »

File negwi-la « filer l'aiguille »

Non/lom, le nom/le zom « homme, les hommes »

b) Insertion d'une syllabe complète

En nuvo lamezon « une nouvelle maison 》

De gro lefey « de grosses feuilles »

Gro difil « gros fil »

En defig « une figue »

Le créole louisianais se singularise néanmoins des autres créoles par le degré de variation observable. En effet, alors que les créoles en général ont des formes agglutinées fixes, le créole louisianais exhibe une variation inter- et intra-individuelle dans les cas d'insertion d'attaque, qui représentent également le type de variation présente dans le français louisianais. Neumann (1985), tout comme Klingler (2003), soulignent que cette variation est particulièrement prégnante lorsque l'élément agglutiné concerne la consonne / $\mathrm{z}$ / et ils y voient des restes de processus de liaison avec perte de l'élément agglutiné, ce que Neumann note dans la graphie par un trait d'union.

(Iо) Variation dans les substantifs

nom ; lez-òm

narb ; lez-arb

lot; lez-ot

Il nous semble que ces exemples viennent plutôt conforter une analyse qui propose que tout substantif à attaque vide est stocké dans le lexique sous plusieurs formes, où homme, par exemple, aurait deux entrées /nom/, /zom/ (Lyche 20IO) rejoignant ainsi le français louisianais. Cette solution a d'ailleurs le mérite de ne pas faire du créole louisianais une exception, la structure $\mathrm{CVCV}$ étant normalement obligatoire pour un créole à base française. 
D'autres données du français louisianais viennent encore conforter une analyse qui rejette l'association de la consonne au Mot-I. Dans son corpus rassemblé dans la paroisse Lafourche, Dajko (2009) soumet ses locuteurs à des exercices de traduction. Elle remarque l'émergence d'une construction $[\text { Verbe }+[t] \text { être }]^{14}$

(iI) Construction [Verbe $+[$ t]être $]$

a) Traduction

Your mother will be angry if you come home late

Ta mame va [t]être fâchée si tu viens à la maison trop tard

I don't think we'll be back in time for dinner

Je crois pas quion va [t]être arrivé d'assez de bonne heure pour manger

b) Parole spontanée

Il veut [t]être en lit pour neuf heures

Côté (2005: 73) fait état d'une lexicalisation identique, mais a priori plus généralisée, dans le français canadien : « La fréquence de la combinaison $[\mathrm{t}]+\hat{e}$ tre semble avoir abouti, en français canadien, à la lexicalisation de la forme /tø:tr/, utilisée en alternance avec / $/$ :tr/ dans de nombreux contextes où un $[\mathrm{t}]$ de liaison n'est nullement motivé. On entend donc fréquemment jai failli [t]être, j’voudrais pas [t]être, tu peux ben [t]être, etc. ». Dans le français louisianais, la variante $[t]$ être ne peut se produire qu'après un verbe modal, mais il s'agit bien d'un processus similaire qui fixe la consonne de liaison dans le Mot-2. Selon Côté (2005 : 73) « [...] en situation de sous-apprentissage ce blocage [est] généralisé et [...] les apprenants conservent les variantes lexicales à initiale consonantique ». Côté propose que le mitchif subit le même phénomène, ce que Papen (20I4) démontre à partir d'une étude lexicale extensive. Le mitchif (langue mixte bilingue français-cri parlée dans l'ouest canadien) atteint un degré de lexicalisation plus avancé des mots liaisonnants que le français louisianais : ours par exemple se réalise indifféremment nuur, luur, zuur ${ }^{15}$ après déterminant défini ou indéfini, singulier ou pluriel (Papen 20r4). Une telle

I4 Je tiens à remercier Nathalie Dajko de m’avoir présenté ces données tirées de son corpus. Sa grande connaissance du terrain louisianais nous a été précieuse.

is La graphie indique une voyelle normalement longue (Papen, c.p.) 
variation est également caractéristique de la langue des enfants. Toutes les études concordent sur la présence dans le lexique des enfants de 2-3 ans de plusieurs variantes qui encodent dans le Mot-2 la consonne de liaison (Chevrot et al. 2009) : éléphant, néléphant, léléphant, zéléphant. Ce n'est que plus tard que la consonne de liaison se détache du Mot-2 pour acquérir une certaine autonomie. Côté attribue la lexicalisation des variantes en mitchif à un contact réduit avec des populations francophones. Nous avons vu grâce au français louisianais que l'illettrisme des locuteurs participait de surcroît à ce phénomène.

\section{Conclusion}

L'étude de la liaison dans une population non lettrée comme celle de la Louisiane converge vers la nécessité d'un traitement pluriel. Les données dont nous disposons ne sauraient être traitées exclusivement par le biais d'une consonne flottante attachée au Mot-I ; elles sont en revanche compatibles avec une analyse par épenthèse ou par allomorphie. Il nous semble avoir montré que la solution la plus improbable plaçait la consonne dans le Mot-I. Cette possibilité écartée, un ensemble de solutions restent disponibles : nous favorisons une approche morphologique pour la liaison pluriel selon laquelle la consonne / z/ est insérée en début de mots à initiale vocalique au sein d'un groupe prosodique étroit. La présence généralisée d'un accent lexical autonomise l'unité lexicale et supprime la possibilité de liaisons postposées à une catégorie principale (Klingler et Lyche 2012). La liaison après le déterminant indéfini et après la préposition en (très fréquente mais non catégorique) peut être analysée à l'aide d'une règle d'épenthèse, tout comme la liaison variable après l'auxiliaire est dans la construction [EST + participe passé]. Nous avons également mis à jour l'existence d'une construction [verbe $+[t]$ être $]$. Le système de liaison dans une population non-lettrée est donc plus restreint que celle d'une population lettrée et en particulier, le domaine des liaisons variables se trouve fort réduit. 
La nature du terrain linguistique en Louisiane ne nous autorise pas à opposer les données de locuteurs non-lettrés à celles de locuteurs lettrés, mais le terrain africain s'y montre plus propice. Boutin et Lyche (2014) comparent une population ivoirienne de lecteurs et de non-lecteurs et observent un système identique de liaisons dans les deux groupes. On relève par exemple chez lecteurs et non-lecteurs confondus des suites comme je suis [z]arrivé, relativement rares dans le système hexagonal et inattendues chez un non-lecteur. Le taux de liaisons variables s'avère néanmoins légèrement plus faible chez les non-lecteurs que chez les lecteurs, ces derniers réalisant très peu de liaisons variables lorsque l'on compare leurs productions à celles de locuteurs francophones hexagonaux. Les contextes donnant lieu à des liaisons variables sont cependant beaucoup plus nombreux en Côte d'Ivoire qu'en Louisiane. Notons par exemple la présence d'une liaison entre chez et un déterminant indéfini (chez [z]une fermme là), ce qui ne s'entendra pas en Louisiane. La pratique du français en Côte d'Ivoire fait partie du quotidien des locuteurs, ce qui n'est pas le cas en Louisiane. Langue majoritaire et officielle en Côte d'Ivoire, le français est très minoritaire en Louisiane où les locuteurs n'ont pas accès à la forme écrite de la langue alors que celle-ci reste très présente en Côte d'Ivoire. Les locuteurs ivoiriens non-lettrés sont en contact avec des lettrés et tout naturellement ils acquièrent les habitudes linguistiques de leurs interlocuteurs. Calderone, Laks et Chelata (2014) dans leur étude sur la fréquence de la liaison mettent en évidence que la variation sociolinguistique ne touche pas au cœur des contextes les plus fréquents de la liaison, seuls sont affectés par les variables de l'âge et du niveau d'instruction les usages périphériques peu fréquents. Les données ivoiriennes viennent conforter ce point de vue.

On aurait néanmoins pu penser que l'absence totale de contact avec l'écrit aurait conduit à l'élimination de la catégorie « liaison variable » du français louisianais sur le modèle du créole avec lequel la langue est en contact ou du mitchif. Or nous avons pu observer un maintien de la liaison facultative, certes très appauvri, mais bien réel. Les données louisianaises sont dans leur ensemble compatibles avec une approche lexicale de la liaison qu'elles viennent renforcer. Le rôle maintes fois souligné de la morphologie apparaît ici clairement et une analyse morphologique de la liaison pluriel semble justifiée. Cette variété de français ne saurait être analysée d'un point 
de vue purement phonologique ou autre, elle démontre bien la complexité du phénomène et la nécessité d'une approche non-uniforme. Les études de variétés non standard ont peut-être une portée limitée certes, mais elle interviennent nécessairement dans l'affinement de nos analyses globales.

\section{Références}

Aub-Büscher Gertrud, 1962, Leparler rural de Ranrupt (Bas-Rhin), Paris, Klincksieck. Blainey Darcie, 2013, First to come, last to go : phonological change and resilience in Louisiana Regional French, Thèse de doctorat, Tulane University, New Orleans. Bollée Annegret \& Neumann-Holzschuh Ingrid, 1998, « Français marginaux et créoles », in Brasseur Patrice (Ed.), Français d'Amérique. Variation, créolisation, normalisation, CECAV, Université d'Avignon, I8I-203.

Boutin Béatrice A. \& Lyche Chantal, 20I4, « Ce que nous apprennent les locuteurs non-lecteurs francophones sur la liaison », in Coquillon Annelise et Soum Christiane (Eds) La liaison : approches contemporaines, Berne, Peter Lang, 287-316.

Calderone Basilio, Laks Bernard \& Chelata Chiara, 2014, « French liaison and lexical repository », in Chelata Chiara \& Calamai Silvia (Eds) Advances in Sociophonetics, Amsterdam, Benjamins, 3I-56.

Chaudenson Robert, 1989, Créoles et enseignement du français, Paris, L'Harmattan.

Chevrot Jean-Pierre, Dugua Céline \& Fayol Michel, 2009, « Liaison acquisition, word segmentation and construction in French : a usage-based account », in Journal of Child Language, 36, 557-596.

Chomsky Noam \& Halle Morris, 1968, The Sound Pattern of English, New York, Harper and Row.

Churma Donald G., 1977, « On choosing between linguistic analyses : a reply to Klausenburger », in Lingua, 42, I3I-I52.

Côté Marie-Hélène, 2005, « Le statut lexical des consonnes de liaison », in Langages, 158, 66-78.

Côté Marie-Hélène, 2010, « Le statut des consonnes de liaison : l’apport des données du français laurentien », in Neveu Franck et al. (Eds) $2^{\text {eme }}$ Congrès mondial de Linguistique Française, Paris, Institut de Linguistique Française, I279-I288.

Côté Marie-Hélène, 20II, « French liaison », in van Oostendorp Marc, Ewen Colin, Hume Elisabeth \& Rice Keren (Eds), Companion to Phonology, Malden, WileyBlackwell, 2685-2710. 
Côté Marie-Hélène, 20I2, « Laurentian French (Quebec). Extra vowels, missing schwas and surprising liaison consonants », in Gess Randall, Lyche Chantal \& Meisenburg Trudel (Eds) Phonological Variation in French. Illustration from three Continents, Amsterdam, John Benjamins, 235-374.

Côté Marie-Hélène, 2013, «Understanding cohesion in French liaison », in Language Sciences, 39, 156-166.

Dajko Nathalie, 2009, Ethnic and geographic variation in the Lafourche basin, Thèse de doctorat, Tulane University, New Orleans.

Dejean de la Bâtie Bernadette, 1993, Word boundary ambiguity in spoken French, Thèse de doctorat, Monash University, Victoria, Australia.

De Jong Daan, 1994, « La sociophonologie de la liaison orléanaise, in Lyche Chantal (Ed.), French Generative Phonology : Restrospective and Perspectives, AFLS/ ESRI, 95-130.

Delattre Pierre, 1966, Studies in French and Comparative Phonetics, La Haye, Mouton. Dubois Sylvie, 2003, « Pratiques orales en Louisiane », La Tribune Internationale des Langues Vivantes, 33, 89-95.

Dubois Sylvie, 2010, « Introduction », in Dubois Sylvie (Ed.), Une histoire épistolaire de la Louisiane, Sainte-Foy, Presses Universitaires de Laval, I-I4.

Durand Jacques \& Lyche Chantal, 2003, « Le projet 'Phonologie du français contemporain' et sa méthodologie », in Delais-Roussarie Elisabeth \& Durand Jacques (Eds), Corpus et variation en phonologie du français, Toulouse, Presses Universitaires du Mirail, 213-276.

Durand Jacques \& Lyche Chantal, 2008, « French liaison in the light of corpus data », in Journal of French Language Studies, 18, 33-66.

Durand Jacques \& Lyche Chantal, 2013, « PFC et les français périphériques », in Ledegen Gudrun (Ed.), La variation du français dans les espaces créolophones et francophones. Tome I, Paris, L'Harmattan, II-29.

Durand Jacques, Laks Bernard \& Lyche Chantal, 2009, « Le projet PFC (phonologie du français contemporain) : une source de données primaires structurées », in Durand Jacques, Laks Bernard \& Lyche Chantal (Eds), Phonologie, variation et accents du français, Paris, Hermès, 19-6I.

Encrevé Pierre, 1988, La liaison avec et sans enchaînement. Phonologie tridimensionnelle et usages du français, Paris, Seuil.

Fouché Pierre, 1959, Traité de prononciation française, Paris, Klincksieck.

Gougenheim Georges, 1938, Le système grammatical de la langue française, Paris, Bibliothèque du français moderne.

Klausenburger Jurgen, 1974, « Rule inversion, opacity, conspiracies : French liaison and elision », in Lingua, I4, I67-179. 
Klausenburger Jurgen, 1978, « Liaison 1977 : the case for epenthesis », in Studies in French Linguistics, I/2, I-20.

Klingler Thomas 2003, If I could turn my tongue like that. The Creole Language of Pointe Coupée Parish, Louisiana, Baton Rouge, Louisiana State University Press.

Klingler Thomas \& Lyche Chantal 2012, «'Cajun' French in a non-Acadian community. A phonological study of the French of Ville Platte, Louisiana », in Gess Randall, Lyche Chantal \& Meisenburg Trudel (Eds), Phonological Variation in French. Illustrations from three continents, Amsterdam, John Benjamins, 275-312.

Laks Bernard, 2005, « La liaison et l'illusion », in Langages, I58, IOI-I26.

Lyche Chantal, 2010, « Mot phonologique, mot graphique et liaison dans quelques lettres de Louisiane », in Dubois Sylvie (Ed.), Une histoire épistolaire de la Louisiane, Sainte-Foy, Presses Universitaires de Laval, 27-43.

Nguyen Noël, Wauquier-Gravelines Sophie, Lancia Leonardo \& Tuller Betty, 2007, « Detection of liaison consonants in speech processing in French: Experimental data and theoretical implications », in Prieto Pialr, Mascaró Joan \& Solé MariaJosep (Eds), Segmental and Prosodic Issues in Romance Phonology (Current Issues in Linguistic Theory ; 282), Amsterdam, John Benjamins, 3-23.

Mallet Géraldine, 2008, La liaison en français : descriptions et analyses dans le corpus PFC, Thèse de doctorat, Université Paris Ouest Nanterre La Défense.

Morin Yves-Charles, 2003, « Remarks on prenominal liaison consonants in French », in Stephan Ploch (Ed.), Living on the edge. 28 papers in honour of Jonathan Kaye, Berlin, Mouton de Gruyter, 385-400.

Neumann Ingrid, 2005, Le créole de Breaux Bridge, Louisiane, Hamburg, Helmut Buske Verlag.

Papen Robert, 20I4, « La liaison en mitchif: un cas d'acquisition incomplète fossilisée ? », in Coquillon Annelise \& Soum Christiane (Eds), La liaison : approches contemporaines, Berne, Peter Lang, 213-238.

Papen Robert \& Rottet Kevin 1997, «A Structural Sketch of the Cajun French Spoken in Lafourche and Terrebone Parishes », in Valdman Albert (Ed.), French and Creole in Louisiana, New York, Plenum Press, 71-108.

Schane Sandford, 1968, French Phonology and Morphology, Cambridge (MA), MIT Press.

Selkirk Elisabeth, 1972, The Phrase Phonology of English and French, Thèse de doctorat, MIT.

Soum Christiane, 1997, L'apprentissage de l'écriture, contraintes orthographiques et contraintes orales, Thèse de doctorat, Université de Toulouse-Le Mirail.

Soum Christiane \& Coquillon Annelise (Eds), 20I4, La liaison : approches contemporaines, Berne, Peter Lang. 
Spinelli Elsa, Cutler Ann \& McQueen James M., 2002, « Resolution of liaison for lexical access in French », in Revue française de phonétique appliquée, 7, 83-96.

Ternes Elmar, 1977, « Konsonantische Anlautveränderungern in den keltischen und romanischen Sprachen », in Romanistisches Jahrbuch, 28, 19-53.

Valdman Albert, 2007, « Variation et revitalisation du français en Louisiane », in Ayres-Bennett Wendy et Jones Mari C. (Eds) The French Language and Questions of Identity, Oxford, Legenda, I9I-205.

Valdman Albert, Rottet Kevin, Ancelet Barry, Guidry Richard, Klingler Thomas A., LaFleur Amanda, Lindner Tamara, Picone Michael D. \& Ryon Dominique, 2010, Dictionary of Louisiana French as Spoken in Cajun, Creole, and American Indian Communities. The University Press of Mississippi.

Wauquier-Gravelines Sophie \& Braud Virginie, 2005, « Proto-déterminant et acquisition de la liaison obligatoire en français $\gg$, in Langages, I58, 53-65. 


\section{Ecrits en contexte de surdité : de la variation et de ses spécificités}

\section{Introduction}

L'analyse de SMS rédigés par des sourds permet de mettre en relief la complexité à l'œuvre dans ces écrits, tant du point de vue des formes (abréviations, etc.) que du point de vue des contacts de langue. L'analyse des pratiques de différents scripteurs sourds de la région rouennaise et de la Réunion a permis de mettre au jour à la fois la complexité à l'œuvre dans les écrits SMS indépendamment du statut du scripteur (i.e. les marqueurs que les sourds et les entendants partagent) et quelques aspects de la complexité des SMS spécifiques au contexte de surdité, ou «pi-sourd $»^{1}$.

Ainsi, nous avons constaté que la plupart des formes observées dans ces SMS et connues dans la communication médiée par téléphone et ordinateur (cf. Panckhurst 1997) - telles que les abréviations, l'orthographe abrégée, la syntaxe agrégative - étaient communes aux sourds et aux entendants. Néanmoins, nous avons proposé qu'une minorité de phénomènes peut être mise en relation avec la surdité des scripteurs. Les spécificités « sourdes » que nous avons relevées sont liées aux contacts de langues dans le répertoire langagier des sourds sollicités, et à leur accès au français. Ces particularités font souvent l'objet de discussions (quant à leur statut spécifique ou non)

I Cette expression provient de l'emprunt en français d'un signe LSF signifiant 'typique' ou 'propre à, cf. Blondel Marion, Gonac'h Jeanne, Ledegen Gudrun \& Seeli Julia, 20II, « Existe-t-il des SMS pi-sourds ? », 20II, Conférence à la FLSH à l'Université de La Réunion : <http://www.sites.uhb.fr/webtv/appel_film.php?lienFilm=825>. 
lorsqu'elles sont soumises à l'évaluation des experts de l'écrit ou à l'évaluation des scripteurs eux-mêmes.

En effet, l'« écriture SMS » peut être évoquée pour expliquer certaines abréviations et style télégraphique choisis par les scripteurs sourds dans nos données : il en est ainsi de l'omission de petits mots-outils (déterminants, prépositions), qui s'inscrit dans la logique de la syntaxe agrégative (Koch \& Oesterreicher 1990 ; Raible 1992), ou encore une syntaxe caractéristique du français parlé (dislocation à droite ou à gauche, clivée ou pseudo-clivée : Dit moi vite quel nom le cd que j ai oublié dans la voiture $^{2}$ (SMS, sourd, Réunion). Dès lors, comment décider qu'une forme caractérise la surdité du scripteur ? Parmi les pistes de recherche qui s'offraient à nous, deux nous semblaient intéressantes : d'une part échanger avec les scripteurs au sujet des catégories repérées par les experts et ainsi distinguer celles qui coïncident entre experts et scripteurs, celles qui font débat; d'autre part étendre l'analyse à d'autres activités littéraciques (Street 2000) afin de relever les mêmes types de formes «pi-sourd » dans d'autres types d'écrits.

Après avoir échangé avec certains des informateurs des corpus SMS, il est apparu que la première option était délicate et qu'il fallait mettre en place de nouvelles stratégies pour engager une approche métalinguistique en LSF. En effet, lorsque nous avons soumis une dizaine d'exemples de SMS à l'une de nos informatrices en lui posant une question très générique sur ce qui lui apparaissait spécifique à l'écrit des sourds, une seule forme a été retenue : « les vac' » pour « les vacances ». La réduction du mot par l'apostrophe a ainsi été le seul indice de surdité relevé par l'informatrice. Dans la suite de l'entretien plus orienté, et dans la mesure où la plupart des formes qui retiennent notre attention sont des formes non normées, l'informatrice nous disait : « ah oui les sourds ont des difficultés en français $»^{3}$. La discussion s'orientait ensuite sur les difficultés en général et les

2 Les exemples de SMS et des copies sont repris dans leur orthographe originale.

3 S'agissant du non-standard, cette difficulté est d'autant plus amplifiée, cf. le « principe de formalité de Labov : « Any systematic observation of a speaker defines a formal context in which more than the minimal attention is paid to speech. » (1973: 113). 
motifs des difficultés des sourds à l'écrit. Nous n'avons donc pas retenu cette option méthodologique. L'insécurité scripturale de l'informatrice s'est ici révélée sur fond de pratiques SMS qui sont fortement méconsidérées dans la société française, parangon de l'« idéologie du standard » (Milroy \& Milroy 1985). La deuxième option a été rendue possible pour l'un de nos scripteurs. Si ce n'est à l'ensemble de ses pratiques de l'écrit, nous avons eu l'occasion d'accéder à des écrits très différents des SMS : ses copies d'examen de première année à l'université.

\section{Hypothèses}

Les copies d'examen et les SMS constituent des types d'activités littéraciques opposées du point de vue de l'autocontrôle et de la norme prescriptive. Leur analyse contrastive, devrait donc souligner plusieurs différences.

I) Les zones de variation relevées dans les SMS devraient être moins nombreuses dans les copies, mais certaines devraient résister et nous permettre de mettre au jour les formes relevant spécifiquement des scripteurs sourds. En effet, certaines des formes dans les SMS relèvent de la syntaxe agrégative, d'autres relèvent de zones instables attestées dans les pratiques de locuteurs $\mathrm{L}_{2}{ }^{4}$, d'autres sont liées au phénomène de circulation avec le créole ou la LSF. Enfin, on peut relever des combinaisons des catégories mentionnées (Gonac'h et al. 2012)5.

4 «Les difficultés des sourds à maîtriser le français sont très proches de celles que rencontrent les apprenants de langue seconde et produisent la plupart du temps les mêmes effets, c'est-à-dire les mêmes erreurs » (Dubuisson et al. 1998 : 193).

5 Quand les spécificités sont attestées régulièrement auprès de différents profils de locuteurs sourds (pratiquant ou non la LSF, ayant ou non une grande compétence en français, ayant appris tardivement ou non le français ...), ces spécificités deviennent régularités et nous les répertorions parmi les traits pi-sourds. Ces traits nous renseignent sur l'organisation spécifique que la surdité entraîne (cf. plus bas) : choix 
2) Les formes hypercorrigées, liées à l'insécurité linguistique des scripteurs sourds et à l'« autodépréciation de [leurs] propres pratiques » (Estève \& Millet 20II : 284) devraient être plus fréquentes dans les copies d'examen (Ledegen 2000) que dans les SMS. Ainsi, on peut supposer que nous observerons non pas l'omission mais l'ajout de mots-outils, indice éventuel d'hypercorrection ${ }^{6}$.

3) Les absences de certains éléments attendus dans des contextes spécifiques pourraient signaler des stratégies d'évitement, selon les contextes d'écriture.

\section{Méthodologie}

Nous avons travaillé sur II 7 SMS et Is copies d'examen d'une même scripteure (réunissant respectivement II34 mots et 4020 mots). Cette analyse nous permet de nous concentrer sur les pratiques d'une personne en particulier et de faire une étude de cas exploratoire. Notre travail sur d'autres corpus SMS (Blondel et al. 20II, Ledegen et al. 20II, Gonac'h et al. 20I2) et sur des copies d'examen (Gonac'h \& Mortamet 20II) ou des situations de tests (Ledegen 2000) nous permet de repérer et d'interroger les spécificités de ce petit corpus au regard des autres données recueillies préalablement.

des temps ; ordre des mots dans la phrase ; omission, ajout et substitution dans les mots-outils, etc. (Dubuisson et Daigle 1998 ; Tuller 2000 ; Garcia et Perini 2010).

6 En effet, si nous suivons une définition élargie de l'insécurité linguistique - « substituer plus ou moins systématiquement une forme $\mathrm{X}$ à une autre forme $\mathrm{Y}$, qu'il croit à tort ou à raison, apte à susciter chez le destinataire des jugements de valeur plus favorables $\gg$ (Berrendonner 1998:89) - nous pouvons considérer certaines occurrences à la lumière de cette attitude (qui revient souvent à privilégier « la forme la plus compliquée [qui] est la meilleure », cf. Paveau 20ı0). 


\section{Du « de » instable comme indice d'insécurité}

Qu'il soit préposition, déterminant ou subordonnant, de est extrêmement instable à la fois dans le corpus des SMS et dans le corpus des copies d'examen. Dans le Tableau i, nous avons recensé les différentes occurrences ou omissions de de : nous avons distingué les cas où il était omis (codé [...]) des cas où il était utilisé à la place d'un autre (substitution : codé de en gras) ou ajouté (ajout : codé en italiques).

Dans les deux contextes d'écriture, SMS et copies d'examen, de se révèle instable ${ }^{7}$ : tantôt absent, tantôt utilisé à la place d'un autre mot-outil, tantôt ajouté. Quand il est omis, cela peut s'expliquer par sa « légèreté » phonétique et sémantique (Abeillé \& Godard 2004), et par la logique de la syntaxe agrégative dans le contexte SMS plus particulièrement. Dans les exemples (I) et (IO), de n'est pas réalisé en tant que préposition respectivement dans le GN ou le GPrép ; dans les exemples (2) et (3), en tant que conjonction : risquer + Inf, essayer + Inf.

Quand il est utilisé à la place d'un autre subordonnant (en l'occurrence $\dot{a}$ dans les exemples 4 et 12 : apprendre à, être forte $\dot{a}$ ), on peut supposer qu'il est choisi parmi d'autres soit par un procédé analogique (ex. I2 : je suispas forte de conduire en karting et $j$ ai peur de conduire), soit parce qu' il est un mot outil passe-partout, sémantiquement peu précis. Pour l'exemple (II), on peut l'analyser comme l'utilisation d'un déterminant partitif, ou encore comme un télescopage avec l'expression d'habitude.

Enfin, quand de est ajouté, il peut aussi appartenir à différentes catégories (déterminants partitifs pour des définis dans les exemples (7) et (8), prépositions dans le groupe adjectival (9) ou nominal (13) ou insérés comme conjonctions entre deux verbes qui ne le sous-catégorisent pas en (5) et (6).

Ainsi, dans toutes les catégories d'écarts identifiées (omission / substitution / ajout), de est concerné par les mêmes catégories lexicales : déterminant, préposition, conjonction.

7 Soulignons tout de même que la majorité de ses emplois - en tant que déterminant, préposition ou conjonction - sont standard : il en est ainsi de 39 occurrences dans les copies d'examen par exemple. 


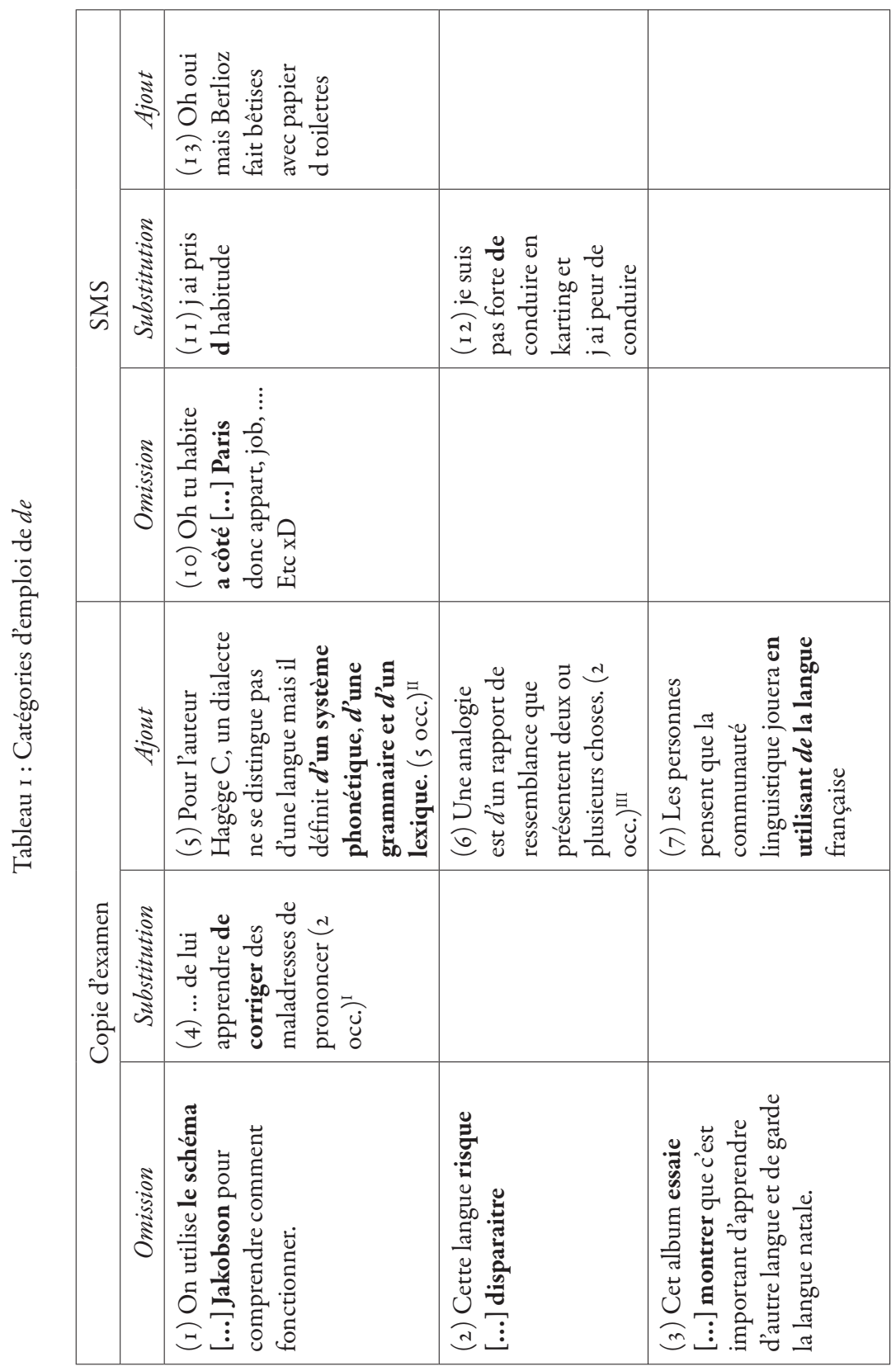



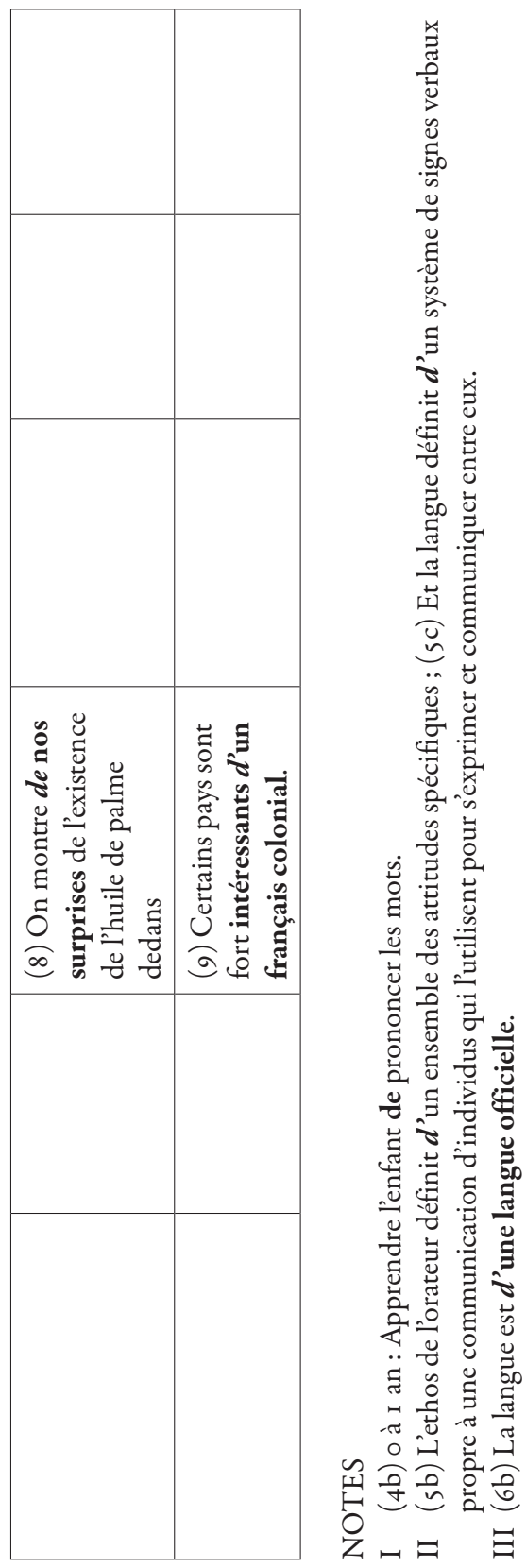
Toutefois, nous avons relevé plus d'ajouts dans les copies d'examen que dans les SMS. Même si le corpus ne peut pas être qualifié de quantitatif, il nous sert de laboratoire pour tester ces hypothèses. Nous touchons ici, nous semble-t-il au phénomène d'hypercorrection, tel que défini par Berrendonner (1998, cf. note 6). En effet, si l'on reprend ses quatre principes qui régissent les phénomènes d'hypercorrection, il apparait que cet ajout de de correspond bien à un phénomène de décoration, d'ornementation : ainsi, d'abord, « le champ de variation peut préexister en langue » (1998: 96). Dans le cas de ce petit mot de, le champ de variation est important (ex : concurrence entre à et de dans différents contextes « populaires »: le père / la mère à quelqu'un; aller au médecin). Même si elle n'est pas concurrente dans le contexte où nous la relevons ici, la variante de semble bien avoir plus de prestige que celle en à puisque cette dernière est très peu ajoutée dans les copies. Le scripteur observé semble bien opérer un choix et de est toujours privilégié donc « hiérarchisé dans les impératifs langagiers 》 (1998 : 96). Pour autant, cet ajout du de n'a donc pas l'effet souhaité par le scripteur puisqu'il ne correspond pas à la norme attendue dans la copie.

Notons par ailleurs que le cadre contraint de l'écrit universitaire pourrait être à l'origine des exemples (5) et (6) contenant un ajout de de-définir de pour se définir par et être de pour être - qui semblent réunir la caractéristique d'être un technolecte des analyses linguistiques ou sociolinguistiques ${ }^{8}$.

\section{Des « cousins $\gg$ lexicaux}

Comme nous l'avions relevé dans d'autres données (Blondel et al. 20II), le scripteur utilise ici des mots de la même famille lexicale (des « cousins ») à la place de ceux qui sont attendus. D'autres études traitant de l'écrit des

Nous avons par ailleurs relevé des créations morphologiques et morphographiques telles que éconination ou co-éconination pour énonciation. On relève également publiquer pour publier. Ainsi, comme dans le travail de Daigle et al. sur les pseudomots, notre scripteure a « accès au traitement sublexical de nature morphologique, ce qui indique une sensibilité à certaines règles de formation des mots du français » (2006: 100). 
sourds mentionnent ce type de substitution : « un pays imagination [pour] (imaginaire) $\gg($ Garcia \& Perini 2010:87; Vanrullen et al. 2012). Cette substitution entre catégories lexicales concerne les échanges nom/adjectif ou adjectif/nom, à l'exception d'une occurrence de verbe pour un nom. C'est ce qu'illustre le Tableau (2) pour les copies d'examen d'une part, et les SMS de l'autre.

Tableau 2 : Substitutions entre catégories lexicales

\begin{tabular}{|c|c|c|c|c|}
\hline \multicolumn{3}{|c|}{ Copie d'examen } & \multicolumn{2}{|c|}{ SMS } \\
\hline Adj pour $N$ & $\mathrm{~N}$ pour Adj & $\mathrm{V}$ pour $\mathrm{N}$ & Adj pour $N$ & $\mathrm{~N}$ pour Adj \\
\hline $\begin{array}{l}\text { ( I 4) Cet album } \\
\text { fait comprendre } \\
\text { que chaque } \\
\text { langue a de } \\
\text { valeur pour la } \\
\text { populaire }\end{array}$ & $\begin{array}{l}\text { (1 7) La } \\
\text { société rend } \\
\text { la possibilité } \\
\text { d'être } \\
\text { bilinguisme }\end{array}$ & $\begin{array}{l}\text { (20) On } \\
\text { préjuge } \\
\text { sur l'huile } \\
\text { de palme. }\end{array}$ & $\begin{array}{l}(2 \text { I })[\ldots] \\
\text { C'est vrai } \\
\text { l universitaire } \\
\text { c est dur ... }\end{array}$ & $\begin{array}{l}\text { (23) donc tu es } \\
\text { plus courage que } \\
\text { moi }\end{array}$ \\
\hline $\begin{array}{l}\text { ( I } 5 \text { ) En XIXè } \\
\text { siècle, le français } \\
\text { a été utilisé le } \\
\text { plus pour les } \\
\text { pays à cause des } \\
\text { coloniaux donc } \\
\text { certains pays } \\
\text { utilisent la langue } \\
\text { maternelle ou la } \\
\text { langue choisie. }\end{array}$ & $\begin{array}{l}\text { ( } 18 \text { ) Chaque } \\
\text { région de la } \\
\text { France a des } \\
\text { différences a } \\
\text { accents. }\end{array}$ & & $\begin{array}{l}\text { (22) Mdrun } \\
\text { chaton plus } \\
\text { joueux }\end{array}$ & $\begin{array}{l}\text { (24) Je confiance } \\
\text { pas }\end{array}$ \\
\hline $\begin{array}{l}\text { ( } 16 \text { ) Une } \\
\text { analogie est } \\
\text { d'un rapport de } \\
\text { ressemblance } \\
\text { que présentent } \\
\text { deux ou plusieurs } \\
\text { choses ou } \\
\text { personnes et la } \\
\text { vraisemblable } \\
\text { qu'on utilise pour } \\
\text { convaincre }\end{array}$ & $\begin{array}{l}\text { ( I 9) Il y a des } \\
\text { différences } \\
\text { idées des } \\
\text { auteurs }\end{array}$ & & & \\
\hline
\end{tabular}


Un adjectif pour un nom (Adj pour $\mathrm{N}$ )

Pour les exemples (I4)populaire (pour le peuple) et (2I) universitaire (pour l'université), on pourrait supposer que le suffixe -aire n'est pas reconnu comme une forme typique de l'adjectif et est utilisé comme marqueur de nom, à l'instar des destinataire, documentaire, bibliothécaire. On peut aussi penser qu'il s'agit d'un cas d'adjectif substantivé (la (chose) populaire; la (chose) universitaire), logique à laquelle on peut rattacher l'exemple (I6): la (chose) vraisemblable pour vraisemblance.

Dans la même logique, le néologisme joueux de l'exemple (22) vient désambiguïser le terme joueur qui peut être nom ou adjectif.

Enfin, (15) atteste coloniaux pour colonies, sans que l'énoncé soit agrammatical, l'adjectif substantivé pouvant désigner les personnes établissant les colonies dans ce cadre d'analyse socio-historique de la langue française (même si l'usage consacré sera colonies, de fait).

Un nom pour un adjectif ( $\mathrm{N}$ pour $\mathrm{Adj})$

Les exemples (17) à (19) révèlent des noms employés pour des adjectifs, au sein d'un paradigme clair pour (17), mais de façon plus complexe pour (18) et (19) car les deux interprétations sont plausibles : adjectif (différents $N$ ) ou nom (des différences de $N$ ).
(17) la possibilité d'être bilinguisme
(17') la possibilité d'être bilingue
(I8) Chaque région de la France a des différences accents
(18') Chaque région de la France a différents accents
(I8") Chaque région de la France a des différences $d$ 'accents
(19) Il y a des différences idées des auteurs
(19') Il y a différentes idées des auteurs
(19") Il y a des différences d'idées des auteurs

De la même manière, dans (23), le nom courage est réalisé dans le paradigme de l'adjectif de même famille lexicale, courageux. Pour l'exception de l'échange Nom/Verbe de l'énoncé (24), il semblerait que nous ayons ici à 
faire à un calque de la LSF (24' et Figure I), avec la réalisation d'un lexème valant autant pour un prédicat incluant un adjectif (je ne suispas confiante) que pour un prédicat incluant un nom (je n'ai pas confiance)?.

(23) donc tu es plus courage que moi

(23') donc tu es plus courageux que moi

(24) je confiance pas

(24') Pointé-vers-soi

(AVOIR-)CONFIANCE

Négation-index

«Première personne »

« avoir confiance, être confiant »

« ne pas »

\section{Un Verbe pour un Nom (V pour N)}

Comme pour l'exemple adjectival (22) «joueux », les copies révèlent une création verbale «préjuger » en (20). On peut y voir une lecture littérale, presque étymologique, dans la logique de certains usages L2 (20), tout comme un glissement catégoriel (20') :

(20) On préjuge sur l'huile de palme : au sens de « juger avant »

(20') On a des préjugés sur l'huile de palme > on préjuge sur l'huile de palme.

En résumé, les « cousins » catégoriels concernent autant les noms utilisés pour les adjectifs, que l'inverse, mais sont plus fréquents dans les copies d'examen que dans les SMS. Le contexte formel convoque un technolecte universitaire (analogie, vraisemblance, bilinguisme, colonies) plus propice à la confusion entre classes lexicales, et les néologismes semblent prendre de l'ampleur dans le même mouvement que l'hypercorrection (cf. plus haut, note 8 ).

Par ailleurs, la distinction verbo-nominale est loin de faire consensus dans les descriptions linguistiques des langues des signes. En LSF, un inventaire limité de paires nom/verbe est proposé dans Cuxac (1996) et fondé sur un contraste articulatoire (mouvement court et répété pour le signe

9 Cf. plus loin, où nous revenons sur la distinction verbo-nominale qui semble non pertinente dans le lexique de la LSF. 
à traduire par « chaise » versus plus ample et simple pour « s'asseoir ») mais ces contrastes phonologiques sont parfois neutralisés en distribution dans un énoncé. Pour Risler (2008: 94), c'est selon le marquage morphologique et la construction syntaxique qui est appliquée aux signes qu'on peut différencier les noms des verbes et la notation à l'aide de la langue vocale environnante biaise la lecture parce que l'on associe « implicitement les signes traduits par des noms à des noms en LSF, et les signes traduits par des verbes à des verbes en LSF. » (Risler 2008 : 89). Ainsi, « le signe [malade] pointer vers une personne et signer [malade] sera glosé en [lui] - [malade], ou [là] - [maladie] » (Risler 2008: 89).

\section{Des «évités»}

Il est intéressant de noter que nous ne relevons, chez notre scripteure, de pronoms compléments de troisième personne (lui; le; la), ni dans les copies ni dans les SMS. On peut penser que cette absence de pronoms est liée à une sorte d'évitement de formes qui sont considérées comme des pièges par le scripteur en insécurité. En effet, dans un article précédent, nous avions relevé dans nos corpus beaucoup de variation dans cette zone (Blondel et al. 20II), comme le montre aussi l'exemple (25) de notre scripteure.

\section{(25) C bizarre pckj'ai jamais vu comme ça déviter avec des autres chats \\ (25’) C bizarre pck je l'ai jamais vu comme ça}

Il arrive également que la répétition du nom soit préférée à l'utilisation d'un pronom. La répétition de «new lunettes » dans l'échange (26) entre A (notre scripteure observée dans cet article) et B (son interlocuteur) en témoigne. Cette même stratégie est adoptée tant dans le contexte prescriptif des examens que dans le contexte spontané de l'écriture SMS. Dans les deux situations, nous attestons ainsi des redondances et répétitions, donnant une impression de longueur, comme par exemple dans le SMS (27).

(26) A : On revient a I février a Caen pour surveiller et chercher new lunettes B : Dakkk enfin!!

A : Ha oui ça fait $d$ bien 
B : ok hihihi

A : T verras qu j serai moche avec new lunettes

(27) Mais nous sommes chez moi à [lieur]. et on ne peut pas aller à [lieu2] car [nomı ] travaille. et on viendra à [lieu2] en février car [nomı] sera en vacances pendant un mois cool gros bisous (SMS sourd, Normandie)

Il est ainsi intéressant de voir réunis, par le biais de cette même stratégie d'évitement, dans des contextes de production pourtant contrastés du point de vue du registre et de la surveillance, ces deux phénomènes : l'absence de pronoms complément de troisième personne qui caractérise habituellement un registre informel (Lambrecht \& Lemoine 1996) et la répétition des groupes nominaux qui caractérise habituellement un registre formel.

\section{La question de l'orthographe}

L'analyse de l'orthographe dans les SMS est difficile car les smart phones, comme leur nom l'indique, sont dotés de fonctions « intelligentes » qui permettent au scripteur de corriger son orthographe, voire de sélectionner un texte prérédigé. L'analyste qui n'a pas accès à toutes les stratégies du scripteur, mais simplement au SMS écrit fini, ne peut savoir si le scripteur maitrise l'orthographe de l'ensemble des mots écrits. Dans le corpus des SMS de notre scripteure, il est donc difficile de savoir dans quelle mesure elle utilise son correcteur orthographique et de connaître son orthographe manuscrite.

Mais, si le corpus de SMS est malaisé à analyser du point de vue de l'orthographe, ce n'est pas le cas des copies d'examen qui sont le lieu par excellence de la pression normative, notamment sur l'orthographe ${ }^{10}$. L'analyse des

M.-A. Paveau relève toutefois que la surveillance semble de mise dans les écrits par clavier (ordinateur et téléphone) :

« Les scripteurs outillés par clavier manifestent une surveillance langagière qui semble supérieure aux scripteurs manuscrits spontanés ; contrairement aux idées 
erreurs d'orthographe dans les copies de notre scripteur révèle que celles-ci sont peu nombreuses si on les compare à d'autres copies d'examen d'étudiants du même niveau. Nous faisons ici l'hypothèse que le mode d'accès au français - essentiellement visuographique - joue un rôle majeur sur la maitrise de l'orthographe : aucune erreur sur les lettres hors système $(y$ grec, par exemple dans étymologique) et de rares erreurs liées au choix des morphogrammes grammaticaux liés au genre (l'expression figé ; les traces ont laissées).

\section{5. « Mauvais genre »}

En ce qui concerne le genre (relevant donc de la morphologie), nous attestons (cf. Tableau 3) des variations ponctuelles, analogues aux difficultés rencontrées par des apprenants $\mathrm{L}_{2}$; en effet, le genre ne correspond plus à une marque systématique ou une notion « saisissable $»^{11}$, et il n'existe pas de distinction de genre en LSF.

Remarquons à l'exemple (3I) que la scripteure ne tranche pas mais écrit les deux genres : «le la grenouille ».

reçues, c'est plutôt l'écriture électronique que l'écriture manuscrite qui conserve la norme académique, en tout cas dans les contextes analysés ici. La rapidité et la simplicité de la frappe laisse sans doute davantage de temps aux scripteurs au clavier de se poser les questions qui mènent à l'hypercorrection [dans l'utilisation de l'accent circonflexe] $\gg($ Paveau $2010: 8 \mathrm{I})$.

II «En indo-européen, la grande opposition de genre qui organisait la langue était celle de l'« animé » et de l'« inanimé », qu'exprimait la distinction du masculinféminin d'un côté, du neutre de l'autre. Et puis cette opposition a perdu tout son sens dans les langues modernes, même celles qui ont conservé un neutre, et aujourd'hui un Allemand ne saurait dire pourquoi Bank est féminin, Boot neutre, et Berg masculin. Les catégories du féminin et du masculin se sont un temps maintenues pour distinguer certaines notions. Les arbres, par exemple, étaient du féminin, il en reste des traces en occitan : la figuièra, la platana. À son tour, cette fonction distinctive s'est perdue, et l'usage qui fixe le pied au masculin et la main au féminin ne répond à aucune notion saisissable, non plus que de dire en d'autres langues le lune et la soleil. » (Présentation Langue et Cité 24, 2013 : I). 
Tableau 3 : Confusions de genre

\begin{tabular}{|l|l|}
\hline \multicolumn{1}{|c|}{ Copie d'examen } & \multicolumn{1}{c|}{ SMS } \\
\hline (28) Et qui porte une panneau écrite & $\begin{array}{l}\text { (32) oki :) et oui je vais bien et en classe c } \\
\text { est sympa mais dur tu sais que c'le fac ... } \\
\text { Et to ? Tjs contente pour tn travail }\end{array}$ \\
\hline (29) la calque sémantique & \\
\hline (30) la féminin & \\
\hline (3 I ) la langage & \\
\hline (3 I) Ils appelèrent le la grenouille & \\
\hline
\end{tabular}

Il est à noter que les copies d'examens n'attestent que des féminins pour des masculins, tandis que l'unique exemple attesté dans les SMS concerne le cas inverse. Peut-être pourrait-on rattacher cette stratégie aux marques d'hypercorrection dans le maniement du technolecte universitaire (calque sémantique, féminin, langage) où ces termes nouvellement appris seraient majoritairement féminins sous la plume de notre scripteure.

\section{Bilan}

Nos analyses révèlent que le domaine de la morpho-syntaxe apparaît comme le domaine le plus affecté, bien davantage que celui du lexique ou de l'orthographe (cf. Tuller $2000^{12}$ ).

Par ailleurs, elles mettent avant tout en lumière un premier axe qui concerne des zones de variation, attestées dans les deux contextes d'écriture examinés - copies d'examen et SMS :

I2 Dans létude de L. Tuller, le rapport est de 91,5\% (II9 erreurs morpho-syntaxiques) versus $8,9 \%$ (I erreurs lexicales). 
I. les cas d'omission et de substitution de de;

2. les « cousins » lexicaux qui attestent de glissements entre catégories dans la même famille lexicale ;

3. les confusions de genre.

Ces trois cas de figure sont aussi relevés dans les pratiques d'apprenants L2, et nous semblent ainsi davantage relever de difficultés de la langue française que d'un trait spécifique à la surdité, tout comme le soulignent Nadeau et Machabée (1998) dans leur comparaison d'écarts produits par des apprenants sourds ou entendants. Il est intéressant de souligner néanmoins, dans la perspective de la circularité interlangue (Coste 2002), que les distinctions N/V/Adj ou Fem/Masc ne sont pas des notions transposables en LSF, du moins au niveau lexical.

Le deuxième axe attesté dans nos deux corpus, la stratégie d'évitement, nous apparaît comme un peu plus spécifique, ou reflétant un contexte d'acquisition « atypique » (Tuller 2000) : l'omission des pronoms à la troisième personne se révèle une stratégie pour faire face à une insécurité linguistique, pour ce pan particulier de la syntaxe du français. Notre analyse rejoint l'étude de Tuller (2000) sur la morphosyntaxe à l'oral d'un locuteur sourd, qui manifeste une grande maîtrise du français : elle y a révélé de fréquentes omissions « illicites » de pronoms objet (i.e. non attestées dans les études sur le français parlé, nommées « licites $\gg)^{13}$, ce qui renforce notre interprétation d'un évitement d'une zone morpho-syntaxique où notre scripteure sait avoir des difficultés.

Le troisième axe concerne le seul écart entre les deux contextes d'écriture que nous ayons attesté : les ajouts de de dans les copies d'examen nous semblent relever de l'insécurité linguistique provoquée par le cadre de l'examen universitaire, qui se manifeste par ces formes hypercorrigées. Nous pouvons y rattacher par ailleurs les créations néologiques du technolecte universitaire. majorité des erreurs morphosyntaxiques ( $52 / 119$, soit 43,7\% des erreurs morphosyntaxiques), dont 17 cas d'omissions de pronoms ( $14,3 \%)$. 
L'analyse contrastive des SMS et des copies révèle ainsi plutôt moins de différences que ce que nous avions soupçonné initialement. En effet, les points saillants de l'écrit de notre informatrice se retrouvent dans les deux contextes : la maitrise de l'orthographe sémiographique, les instabilités des zones morpho-syntaxiques, l'absence des pronoms compléments. Par ailleurs, l'analyse des copies conforte nos précédentes analyses qui mettaient en relief l'imbrication des phénomènes, et la rareté des formes exclusivement liées à la surdité du scripteur.

\section{Références}

Abeillé Anne \& Godard Danielle, 2004, « De la légèreté en syntaxe », in Bulletin de la société linguistique de Paris, tome XCIX/1, 69-106.

Baude Olivier (Ed. scient.), 2013, Langues et Cité, 24, « Féminin, masculin. La langue et le genre ».

Berrendonner Alain, 1998, « Normes d'excellence et hypercorrections », in Cabiers de linguistique française, 20, 87-10I.

Blondel Marion, Gonac'h Jeanne, Ledegen Gudrun \& Seeli Julia, 2orıa, « EcritureSMS en Métropole et à La Réunion : 'Zones instables et flottantes' du français ordinaire et spécificités du contexte de surdité », in Gilles Col et Sylvester N. Osu (dir.), Transcrire, Écrire, Formaliser - (I) Travaux linguistiques du CERLICO, 24, 5I-70.

Blondel Marion, Gonach Jeanne, Ledegen Gudrun \& Seeli Julia, 2orrb, « Existe-t-il des SMS pi-sourds ? », 20II, Conférence à la FLSH à l'Université de La Réunion : $<$ http://www.sites.uhb.fr/webtv/appel_film.php?lienFilm $=825>$.

Coste Daniel, 2002, « Compétence à communiquer et compétence plurilingue », in Notions en question, 6, II5-I23.

Cuxac Christian, 1996, « Fonctions et structures de l'iconicité des langues des signes », Thèse de Doctorat d'Etat, Université de Paris V.

Daigle Daniel, Armad Françoise, Demont Elisabeth \& Gombert Jean-Emile, 2006, « Apprentissage implicite et traitement morphologique. Le cas d'élèves sourds gestuels », in Daigle Daniel \& Parisot Anne-Marie (Eds), Surdité et société : perspectives psychosociale, didactique et linguistique, Québec City, Les Presses de l'Université du Québec, Collection « Santé et société », 99-II3. 
Dubuisson Colette \& Daigle Daniel (Eds), 1998, Lecture, écriture et surdité, Montréal, Éditions logiques.

Dubuisson Colette \& Daigle Daniel, 1998, « Que peut-on conclure des recherches portant sur l'écriture ? », in Dubuisson Colette \& Daigle Daniel (Eds), Lecture, écriture et surdité, Montréal, Éditions logiques, I3I-I52.

Dubuisson Colette, Vincent-Durroux Laurence \& Nadeau Marie, 199I, « L'enseignement de la langue maternelle aux déficients auditifs », Glossa, 27, 32-37.

Estève Isabelle \& Millet Agnès, 20II, « Transcrire et annoter les relations sémanticosyntaxiques de la multimodalité dans les productions des enfants sourds », in Travaux Linguistiques du CerLiCO, 24, 31-49.

Garcia Brigitte \& Perini Marie, 2010, « Normes en jeu et jeu des normes dans les deux langues en présence chez les sourds locuteurs de la langue des signes française », in Langage et société, $131,75-93$.

Gonac'h Jeanne \& Mortamet Clara, 20II, « Pratiques orthographiques en français d'étudiants étrangers : le cas d'étudiants hispano-américains et afghans », in Tranel (Travaux neuchâtelois de linguistique), vol. 54, I13-127.

Gonac'h Jeanne, Seeli Julia, Ledegen Gudrun \& Blondel Marion, 20I2, « Les contacts du français, du créole et de la LSF dans les écrits-SMS », in CLAIX, 24 , Kriegel Sybille \& Véronique Daniel (Dirs), « Contacts de langues, langues en contact », I7I-186.

Koch Peter \& Oesterreicher Wulfgang, 1990, Gesprochene Sprache in des Romania : Franzözisch, Italienisch, Spanisch, Tübingen, Niemeyer.

Labov William, 1973, « Some principles of linguistic methodology », in Language in Society, I, 97-I2O.

Lambrecht Knud \& Lemoine Kevin, 1996, « Grammaire des compléments zéro en français parlé », in Travaux linguistiques du CERLICO, 9, « Absence de marques et représentation de l'absence », 279-309.

Ledegen Gudrun, 2000, Le bon français. Les étudiants et la norme linguistique, Paris, L'Harmattan.

Ledegen Gudrun, Seeli Julia, Blondel Marion \& Gonac'h Jeanne, 201I, « 'Tu pense quoi mieux ?' De la Normandie à La Réunion, les interrogatives en question dans les SMS en contexte de surdité », in Liénard Fabien \& Zlitni Samir (Eds), La communication électronique : enjeux de langues, Limoges, Lambert-Lucas, 223-234.

Milroy James \& Milroy Lesley, 1985, Authority in language, London, Routledge and Kegan Paul.

Nadeau Marie \& Machabée Dominique, 1998, « Dans quelle mesure les erreurs des sourds sont-elles comparables à celles des entendants ? », in Dubuisson Colette \& Daigle Daniel (Eds), Lecture et surdité : visions actuelles et nouvelles perspectives, Montréal, Les Éditions Logiques, I69-195. 
Panckhurst Rachel, 1997, « La communication médiatisée par ordinateur ou la communication médiée par ordinateur ? », in Terminologies nouvelles, 17, 56-58.

Paveau Marie-Anne, 2010, « Les formes graphiques de l'insécurité: l'exemple de l'accent circonflexe », in Le français aujourd'hui, 170, 71-82. <http://www.cairn.info/ revue-le-francais-aujourd-hui-2010-3-page-71.htm $>$

Raible Wolfgang, 1992, Junktion. Eine Dimension der Sprache und ihre Realisierungsformen zwischen Aggregation und Integration, Heidelberg, Winter.

Risler Annie, 2008, « Les classes lexicales en LSF envisagées à partir de la fonction adjectivale », in Silexicales, 5, Actes du colloque Syntaxe, Interprétation lexique des Langues des Signes mai 2006, Université Lille 3, STL éd., IO3-I26.

Street Brian, 2000, « Literacy events an literacy practices : theory and practice in the New Literacy Studies », in Martin-Jones Marilyn \& Jones Kathryn (Eds), Multilingual literacies, Amsterdam/Philadelphia, John Benjamins, 17-29.

Tuller, Laurie, 2000, « Aspects de la morpho-syntaxe du français des sourds », in Recherches Linguistiques de Vincennes, 20, 143-156.

Vanrullen Tristan, Boutora Leila \& Dagron Jean, 2012, « Enjeux méthodologiques, linguistiques et informatiques pour le traitement du français écrit des sourds », in Actes de la conférence conjointe JEP-TALN-RECITAL 20I2, volume 2 : TALN, $559-566$. 



\section{L'appropriation territoriale par les jeunes à travers l'étude d'un corpus de messages envoyés à la radio Skyrock}

\section{Introduction}

Le phénomène d'appropriation territoriale des jeunes de banlieue en tant que remède à l'insécurité socio-culturelle et langagière est un sujet récurrent en sciences humaines et sociales. Dans les années quatre-vingtdix, ce phénomène a été étudié par l'ethnologue David Lepoutre (1997) dans la cité des 4000 à la Courneuve, dans le département de la SeineSaint-Denis. Depuis, le fameux « neuf-trois » garde un rôle de terrain privilégié des études sociologiques (citons, entre autres, les travaux de Kokoreff (2003) et de Sauvadet (2004) et des études linguistiques (p. ex. Liogier (2006), Jamin (2005), Podhorná (2002), etc.). Ce terrain condense tous les paramètres démographiques que les médias catégorisent comme « déviants » de la moyenne qui alimentent des discussions médiatiques sur l'avenir des « jeunes de banlieue » et qui, souvent, de par ce caractère dit « déviant », alimentent également des clichés et des stéréotypes que la société majoritaire se fait à propos de ces derniers (Boyer \& Lochard 1998). Tout comme ce terrain est une « vitrine médiatisée » de l'exemple-type des banlieues françaises, la « vitrine médiatique » des (jeunes des) banlieues est la radio Skyrock. Dans cette contribution, nous allons discuter la question des pratiques discursives témoignant d'une appropriation territoriale, phénomène caractéristique de la quête d'une identité, en prenant pour base un corpus de messages envoyés par les auditeurs à la radio Skyrock. 


\section{Skyrock et sa libre antenne}

Puisque ses auditeurs sont majoritairement âgés de I2 à 25 ans, Skyrock est une radio dite jeune, majoritairement écoutée dans les grandes villes ${ }^{1}$. À la fin des années 1990, la loi sur les quotas de chanson française à la radio pousse Skyrock à opter pour une programmation de chansons de rap et r'n'b (ce qui permet de diffuser des chansons françaises susceptibles de plaire aux jeunes); ceci est à l'époque un grand pari sur l'avenir en France de cette musique. Progressivement, la radio acquiert une identité perçue par les médias comme celle de la « génération black-blanc-beur », celle des « jeunes issus de l'immigration ». Depuis 1997, l'animateur Difool est la figure emblématique de la station. Pendant l'émission de libre antenne « Radio libre » qu'il anime tous les soirs de la semaine entre 2 rhoo et minuit avec ses acolytes, les jeunes téléphonent à la station dans l'espoir de passer à l'antenne et de pouvoir discuter d'un problème avec l'équipe des animateurs (relations amoureuses, amicales ou avec les parents, problèmes de santé ...). Progressivement, au fil des années, une structure triangulaire (Glevarec 2005: 16), portant le nom de Skysolidarité, s'est mise en place entre les animateurs, les appelants mais aussi les auditeurs qui, s'identifiant aux jeunes qui appellent, envoient des messages (téléphoniques ou via le site Internet de la station) pour commenter ce qui est dit, réagir au problème de l'auditeur et éventuellement le conseiller. Ainsi, chaque soir, au début de l'émission puis plusieurs fois pendant, l'animateur Difool rappelle aux auditeurs le numéro de téléphone pour passer à l'antenne puis les différents moyens d'envoyer des réactions : le numéro de téléphone pour les

I Pour une analyse des profils des auditeurs, voir Glevarec \& Pinet (2009: 216-218): le « profil type » d'un auditeur de Skyrock est un homme entre is et i9 ans, étudiant, avec un niveau d'instruction technique/professionnel, habitant dans une agglomération de plus de 200000 habitants. Sans vouloir faire un amalgame stéréotypé entre auditeurs de Skyrock et jeunes des quartiers d'habitat social, soulignons que, en comparaison avec les autres radios jeunes nationales (Fun Radio, NRJ et Virgin Radio, anciennement Europe 2), Skyrock est la seule à afficher ouvertement que ce profil de jeunes est le bienvenu sur son antenne (Fiévet 2008a : 265-268). 
sms mais également le site Internet de la radio pour les messages ${ }^{2}$. Bien que l'émission de libre antenne reste un moment privilégié d'échanges entre les jeunes et les animateurs, c'est l'identité entière de la station qui est fondée sur ce fonctionnement et c'est à tout moment que les jeunes envoient, à destination des studios, des sms ou des messages via le site de la station.

\section{Les radios jeunes : mise en scène des revendications territoriales}

À partir des années 2000, les comportements sociaux et langagiers des jeunes sur cette radio nationale ont été observés par les sociologues des médias, particulièrement par Hervé Glevarec $\left(2005^{3}\right)$. En 2004, le sociolinguiste Cyril Trimaille constate qu'on peut « observer de très nombreuses occurrences d'éléments devenus emblématiques des Parlers Jeunes Urbains sur certaines radios » (Trimaille 2004: I20) mais il faut attendre quelques années pour que voient le jour des travaux analysant ces parlers jeunes, tout en tenant compte de la spécificité de la situation de communication radiophonique (pour une approche socio-lexicologique, voir Fiévet (2008) puis Vašková

2 Ces dernières années, les moyens de communication entre la radio et les jeunes n’ont pas changé fondamentalement mais ont évolué avec les technologies : en 2003 (année de l'enregistrement du corpus de thèse d'Anne-Caroline Fiévet), le numéro pour envoyer des sms était le $4 \mathrm{I} S K Y$ et l'adresse du site était skyrock.com. Onze ans plus tard, en 20I4, suite à la disparition des lettres accompagnant les chiffres sur la plupart des téléphones, le numéro est devenu le 41759. Du fait du développement des réseaux sociaux, le site skyrock.com est devenu le site de la radio dédié aux rencontres entre jeunes, un autre site a été mis en place parallèlement pour présenter ses activités et donc envoyer des messages aux animateurs : skyrock.fm. Une application sur smartphone a également été créée.

3 Cet ouvrage constitue la synthèse de différents articles sur la question parus à partir de 2003. 
(20II) ; pour une approche discursive voir Sow $\left.(2010)^{4}\right)$. Il est certain que ces travaux souffrent du manque d'informations sur le profil sociologique des jeunes qui passent à l'antenne puisqu'il ne s'agit donc pas de travailler sur un « terrain » à proprement parler mais plutôt sur un espace virtuel où les identités concrètes peuvent être virtualisées à volonté. Cependant, il faut admettre que ce type d'émission permet aux linguistes d'avoir un accès relativement facile aux parlers jeunes mais surtout qu'il s'agit d'un objet social insolite, parce que c'est le seul lieu où des pratiques discursives identitaires propres aux jeunes issus des banlieues (et pas seulement des banlieues) sont accessibles dans l'espace public, chacun d'entre nous, journalistes et publicitaires compris, pouvant allumer sa radio et écouter ce type d'émission. C'est pourquoi, l'obstacle du profil sociologique des locuteurs ne pouvant être contourné puisqu'il est impossible d'avoir accès aux véritables identités des jeunes, nous pensons qu'il ne faut pas pour autant se priver de l'analyse de cet objet social mais qu'au contraire, qu'il faut intégrer ce paramètre afin d'apporter une attention toute particulière à ce phénomène de société, dans le cadre de la sociolinguistique urbaine (Bulot 20II ; Bastian, Bulot \& Burr 2009).

Cet extraordinaire engouement pour les sms dans les années 2000 va de pair, il s'entend bien, avec le développement de l'outil technologique qu'est le téléphone portable, les textos remplaçant peu à peu le célèbre « courrier des lecteurs » de jadis ( $c f$. les travaux de Rui (1995), Pasquier (1999), etc.). Comme l'écrivent Ledegen et Simonin, les technologies de l'information et de la communication « facilite[nt] les 'télé-échanges' » et « génèrent une expansion et une diversification des contacts sociaux, des contacts langagiers $\gg(2010: 107)$.

Le phénomène des sms étant relativement récent et dynamique pour des raisons de convergence numérique (Fairon 2010), il est particulièrement attirant pour les chercheurs. Ainsi, de nombreux travaux ont vu le jour ces

4 À notre connaissance, aucune recherche sur cette thématique n’a été menée depuis. Ceci est certainement lié à un certain désintéressement, depuis quelques années, dans les milieux scientifiques, d'un côté pour l'étude des mots identitaires pour les jeunes et de l'autre pour les études sur la radio traditionnelle, du fait de l'avènement du numérique. 
dernières années, mentionnons notamment le projet sms4science initié par le laboratoire CENTAL de l'Université Louvain-la-Neuve (Fairon, Klein \& Paumier 2006). Ils ont ciblé différents enjeux et particulièrement la forme de ces derniers (allant des travaux pionniers d'Anis (200I) à des travaux plus récents, comme p.ex., Cougnon \& Ledegen 2010 ; Panckhurst et al. 2013 ; Bernicot et al. 2014).

\section{Présentation du corpus de messages envoyés à la radio Skyrock}

Le corpus que nous allons analyser sous l'angle de l'appropriation territoriale nous a été rendu accessible grâce au sociologue Hervé Glevarec qui a luimême largement travaillé sur la radio et plus précisément sur la libre antenne (Glevarec 2005) et qui s'est vu confier ce corpus par Skyrock. En contact avec Anne-Caroline Fiévet dans le cadre du Groupe de Recherche et d'Études sur la Radio ${ }^{5}$, il nous a proposé d'en étudier le contenu linguistique. Ce corpus est constitué de 37776 messages envoyés par les jeunes à la station en mars 2005 dans la semaine du 7 au II mars 2005 (toute la programmation, pas seulement la libre antenne). Le tableur comporte plusieurs colonnes à savoir la source (sms, Internet ou i-mode $\left.{ }^{6}\right)$, le pseudo ${ }^{7}$, le département, l'âge, le sexe, le message, la date et l'heure. Concernant la source, sur les 37775 messages, 23179 ont été envoyés via Internet (6I,4\%), I 4433 par sms (38,2\%) et I64 messages via i-mode $(0,4 \%)$. Cette préférence pour Internet est certainement

5 GRER : <http://www.grer.fr/>

6 «nsemble de services et de protocoles permettant de connecter des téléphones portables à internet », utilisé par l'opérateur Bouygues Télécom entre 2002 et 2012. Source : <http://fr.wikipedia.org/wiki/I-mode>

7 Apocope de « pseudonymes », le pseudo est utilisé particulièrement pour désigner les noms dans des univers virtuels comme Internet ou les jeux vidéo. Pour cette raison, nous avons choisi de conserver ce terme (et non d'utiliser pseudonyme), bien qu'il soit familier. 
due au fait qu'en 2005, les sms sont encore souvent facturés à l'unité alors qu'aujourd'hui, les jeunes disposent de forfaits illimités.

Travailler sur un corpus qui date de presque dix ans pourrait sembler peu pertinent pour des recherches sur la dynamique des innovations lexicales, par exemple. Pour nos objectifs, le décalage ne semble pas influencer la façon dont les jeunes auditeurs-scripteurs revendiquent leur identité territoriale. On peut supposer que de la même façon que pour la toponymie, quoique souvent substandard, elle fait partie des éléments les plus stables de la langue. Ainsi, notre prémisse est de considérer que les pratiques de la revendication identitaire à travers l'usage des toponymes vont aller dans le même sens. Ce qui a probablement changé depuis 2005, en revanche, ce sont les pratiques scripturales et leur densité. Aujourd'hui, les formes subissent de moins en moins un abrègement par divers jeux de mots au « squelette consonantique » (Anis 200I), phénomène typique du «parlécrit $\gg(\text { Panckhurst 2006) })^{8}$.

À la différence des corpus recueillis dans le cadre du projet sms4science $^{9}$ par exemple, les sms reçus par la radio Skyrock ont un caractère public puisque leurs auteurs savent que leur message va être affiché sur un ordinateur qui se trouve dans le studio et qu'il est susceptible d'être lu à l'antenne par l'animateur. Avant d'envoyer leur message à la radio, les auteurs ont réfléchi en toute liberté sur la façon de s’identifier (présentation de « soi » avec son vrai prénom ou un pseudonyme) ou non (seul le numéro de téléphone permet ensuite identifier s'il s'agit du même auditeur-scripteur).

8 Note du séminaire du I5/II/20I I à Montpellier (Journées d'étude sud4science: Harmonisation/standardisation des méthodes de traitement de corpus écrits de type sms. Anonymisation, transcodage, annotation) lors de l'exposé de Cédrick Fairon qui observe un certain vieillissement des pratiques scripturales du type « écrit sms » avec le vieillissement de la génération pour laquelle cette pratique de transcodage a été un symbole de la révolte adolescente. Dans une discussion ultérieure, Claudine Moïse, de l'équipe montpelliéraine qui a une collecte en cours en novembre 20II, confirme en revanche que les sms de 20 ir contiennent relativement peu de jeux de transcodage ludique, l'abrégement n'étant plus raisonné par des contraintes pécuniaires et la rapidité de l'écriture étant garantie par les dictionnaires implantés qui proposent la suite du mot qu'on a entamé. 
La même liberté est offerte quant au choix de rester dans un « anonymat du profil sociologique $\gg$ ou de proposer aux destinataires un profil plus ou moins complet (sexe, âge, département) quoique souvent mensonger (notamment pour l'âge), ceci souvent pour une simple raison ludique (parfois ce sont des groupes de pairs qui s'amusent à envoyer les sms lors d'une soirée). En revanche, à la différence des messages envoyés volontairement par les scripteurs dans le cadre de projets scientifiques comme «sms4science », l'envoi d'un message vers le numéro/le site de Skyrock sous-entend un consentement implicite pour passer à la radio ${ }^{10}$. De plus, l'acte d'écriture est spécifique : tout d'abord les récepteurs se situent à trois niveaux, à savoir un animateur / l'équipe ou la radio / la communauté d'auditeurs (Glevarec 2005: 160). De plus, contrairement à un échange classique (échange de sms entre deux amis, par exemple), il n'est pas possible pour les animateurs compte-tenu du nombre de messages qu' ils reçoivent - de tout lire, ce qui apparente ces messages à des sortes de « bouteilles à la mer » et favorise le «flood » c'est-à-dire l'envoi répétitif de messages, dans l'espoir qu'au moins l'un d'entre eux soit lu.

\section{Âge et provenance géographique des scripteurs}

Si l'on exclut les 13622 messages où l'âge n'a été pas indiqué (soit $36,1 \%{ }^{11}$ ), plus de $95 \%$ de messages ont eu comme créateur une personne qui a indiqué avoir moins de 25 ans. 44,6\% des messages qui comportent une

Io Nous remercions Skyrock et particulièrement Pierre Bellanger, Jérôme Aguesse et Olivier Langles pour nous avoir autorisées à accéder à ce corpus. Nous remercions chaleureusement Hervé Glevarec pour sa confiance dans notre travail et pour nous avoir donné accès aux données et à des statistiques de base sur les profils sociologiques des scripteurs. Les statistiques présentées infra s'inscrivent à la suite de son travail présenté dans son ouvrage Libre Antenne (2005 : 155-160).

II Il nous semble intéressant de constater que malgré le chiffre plus élevé des cas où le sexe n’a pas été indiqué ( 14003 sms, soit 37,I\%), l’âge reste la catégorie la plus 
identification d'âge ont été écrits par un «teen-ager », soit une personne entre $13-19$ ans ${ }^{12}$; Hervé Glevarec propose de parler d'un « moment radiophonique adolescent $\gg($ Glevarec $2005: 25)$.

En ce qui concerne le sexe des scripteurs, les plus actifs ont été les garçons (44,3\% de la totalité des messages), notamment ceux âgés de I 6 ans ( $15,5 \%$ des 16747 messages masculins). Pour les filles (I8,6\% de tous les messages du corpus), le pic de l'activité textuelle est plus précoce - à l'âge de Is ans (I 4,8\% parmi les 7025 messages envoyés par les filles). En somme, presqu'une moitié des messages produits par les adolescents sont compris dans la tranche d'âge $15-16$, ce qui nous amène à paraphraser Glevarec en proposant de parler d'《 âge radiophonique scriptural ».

\section{Profil des scripteurs}

Dans le corpus, 13693 messages ont été envoyés sans spécification du département d'origine des scripteurs, soit 36,2\%. Parmi les messages identifiés au niveau territorial ( $63,8 \%$ du corpus), les jeunes de trois départements ont envoyé plus de 1000 messages, à savoir les jeunes de Seine-Saint-Denis $(6,4 \%$ des messages « identifiés »), du Nord $(5,4 \%)$ et de Paris $(4,4 \%)$. En termes de régions, c'est l'Île-de-France qui arrive largement en tête avec $32,4 \%$ des messages (les départements de la Petite Couronne dépassent

soumise à l'anonymisation ou plutôt au « brouillage » volontaire : tandis que l'âge pour les personnes qui n'ont pas voulu avouer leur sexe s'échelonne entre 12 et 36 ans, celui des personnes identifiées par leur sexe va au-delà de ces (probablement véritables) limites et les scripteurs (quasi-exclusivement les garçons) s'amusent plus souvent à mettre des âges plus bas ( $\mathrm{I}-3$ ans) et surtout plus hauts (notamment entre 77 et 99 ans - voir page 64 ).

I2 La jeunesse est une caractéristique des corpus de sms. Par exemple, pour l'étude menée en Belgique en 2004, « 76\% des participants ont moins de 25 ans $\gg$ (Fairon et al. 2006 : 16); pour l'étude menée à Montpellier en 201 « « 80\% ont moins de 30 ans 》 (Panckhurst et al. 2013:2). 
chacun 4\%, les départements de la Grande Couronne dépassent chacun $3 \%)$. Ce sont notamment des grandes agglomérations que les jeunes écrivent le plus souvent (les départements 13 et 69 (soit Marseille et Lyon) ont un score autour de $3,5 \%$, soit environ 850 messages).

\section{Dédicaces et pseudos : mise en mots de l'appropriation territoriale}

Nous allons analyser le corpus selon trois axes : les dédicaces du type en force, big-up et représente et les pseudos, en se concentrant dans les deux catégories uniquement sur ceux qui comportent une composante toponymique.

Tout d'abord, nous avons analysé le corpus du point de vue de trois types de dédicaces à savoir en force, big up et représente. Nous avons choisi ces trois dédicaces parce qu'elles sont emblématiques pour les jeunes et qu'elles sont souvent reprises par les médias ou les humoristes pour stigmatiser les jeunes des quartiers d'habitat social. De notre point de vue, il est alors intéressant de comparer comment l'emploi de ces trois types de dédicaces fonctionne véritablement ${ }^{13}$.

Comme l'écrit Melliani (2000 : I5), l'identité est territorialisée, le territoire étant « le seul espace réellement possédé et maîtrisé, celui de l'enfance et des amis, celui qui préserve de l'humiliation et de la honte ».

I3 Un quatrième type, forever ou for ever («94 FOREVER »), a été écarté du fait du nombre trop peu important d'occurrences (5). Seules les dédicaces à composante identitaire ont été choisies pour l'analyse donc les dédicaces de type standard du type «bisous à, vive le ou même dédicace à» (avec de nombreuses formes orthographiques possibles dont un très fréquent dédicasse mais aussi le squelette consonantique en majuscules $D D K S$ ) ont également été écartées. Notons également que nous n’avons relevé que les dédicaces relevant de l'appropriation territoriale. Les dédicaces peuvent également concerner des personnes (amis ou animateurs de la radio) et même plus généralement la radio ( «SKYROCK EN FORCE »). 
Ces dédicaces en relation avec leurs composantes toponymiques (par exemple « 93 EN FORCE ») sont à la fois des symboles pour les jeunes mais, à la fois également, d'un point de vue extérieur, des stigmates. Les dédicaces avec une revendication symbolique qui font référence au territoire vécu se font le plus souvent par l'intermédiaire d'une simplification pragmatique du système vicésimal complexe (par exemple neuf-quatre, sept-sept, etc.). Ce constat est confirmé dans notre corpus et montre bien le poids du territoire dans les revendications. Nous avons donc classé chaque message en fonction du type de territoire concerné par la dédicace : établissement scolaire (classe, collège, lycée ...), quartier, ville, club sportif ${ }^{14}$, département, région et pays. Le Tableau i montre la proportion de messages incluant un des trois types de dédicaces, en fonction du type de lieu concerné. Notons que c'est la dédicace en force qui est la plus fréquente ( 162 messages alors que big up concerne 63 messages et représente concerne 44 messages). Les lieux les plus souvent cités sont la ville $(27,1 \%)$ et le département $(23,4 \%)$; viennent ensuite les clubs sportifs $(19,7 \%)$ et les établissements scolaires (14,5\%) puis le quartier $(7,4 \%)$ et le pays $(7,1 \%)$. La région est quasi-inexistante ( 2 messages sur tout le corpus $\left.{ }^{15}\right)$.

I4 Sous l'appellation « club sportif », nous regroupons quasi-exclusivement des clubs de football. Cependant, nous avons pu relever une attestation référant à un club de cyclisme, d'où cette appellation générique.

I5 Les deux messages concernent la région Bourgogne. Le premier parait tout à fait sérieux («LES BOURGUIGNON EN FORCE!! » - garçon, I9 ans, du département 2I (donc de Bourgogne). Nous avons comptabilisé le deuxième bien qu'il puisse être interprété de façon ironique : «BOURGOGNE EN FORCE LOL C KOI CE DELIRE DE MEUF EL ET TARE », garçon, I6 ans, du département 76. Ici, n'ayant pas la ponctuation, nous ne pouvons pas savoir si le « LOL » se rapporte ou pas à 《Bourgogne en force ». La non-appartenance du scripteur à la région citée pourrait faire pencher vers l'hypothèse de l'interprétation ironique. 
Tableau I : Proportion de messages incluant en force, big up et représente en fonction des lieux concernés par la dédicace

\begin{tabular}{|c|c|c|c|c|c|}
\hline & en force & bigup & représente & Somme & $\%$ \\
\hline $\begin{array}{c}\text { Établissement } \\
\text { scolaire }^{16}\end{array}$ & I 3 & 22 & 4 & 39 & $14,5 \%$ \\
\hline Quartier & 8 & 6 & 6 & 20 & $7,4 \%$ \\
\hline Ville & 43 & 15 & 15 & 73 & $27, \mathrm{I} \%$ \\
\hline Club sportif $^{17}$ & 50 & I & 2 & 53 & $19,7 \%$ \\
\hline Département & 36 & I I & 16 & 63 & $23,4 \%$ \\
\hline Région & 2 & ० & o & 2 & $0,7 \%$ \\
\hline Pays & IO & 8 & I & I9 & $7,1 \%$ \\
\hline Total & 162 & 63 & 44 & 269 & $100,0 \%$ \\
\hline
\end{tabular}

Les trois types de dédicaces ont été analysés du point de vue du profil des scripteurs. Le Tableau 2 montre la répartition par sexe pour les trois types de messages. Les chiffres concernant le corpus total ont également été rappelés. Les analyses montrent que les dédicaces à composante identitaire territoriale sont écrites par une proportion de jeunes anonymes beaucoup plus faible que pour le corpus total : les profils inconnus représentent $36,1 \%$ du corpus total alors qu'ils représentent seulement $18,5 \%$ de ceux qui ont envoyé une dédicace avec en force, II,I\% de ceux qui ont envoyé une dédicace avec big up et I $8,2 \%$ de ceux qui ont envoyé une dédicace avec représente. Il y a donc une corrélation, somme toute logique, entre le fait

Soit l'établissement est cité comme par exemple «BIG UP AU LYCéE JULES VERNE DE MONDEVILLE » (garçon, I6 ans, du département I4) soit la dédicace est plus précise et mentionne une classe : «BIG UP AU COLLEGE JEAN MERMOZ DE METZ ET SUR TT A LA ${ }_{4} \mathrm{EMME}_{2} \mathrm{KI}$ DECHIRE TT » (fille, I 4 ans, du département 57).

I7 Quand il est difficile de dissocier le club sportif de la ville, c'est le sens global du message qui a été pris en compte. Par exemple, dans le message « MARSEILLE EN FORCE ET NIKE TA MERE PARIS MARSEILLE SON 3 EME ET PARIS I2 EME » (garçon, I7 ans, département 77), nous avons pris le parti méthodologique de comptabiliser « Marseille » dans « club sportif ». 
de s'identifier et le fait de revendiquer une identité territoriale dans son message. Remarquons également que l'écart entre la proportion de filles et de garçons est beaucoup plus faible quand il s'agit des dédicaces. Si l'on observe les chiffres pour le nombre total de messages envoyés, les garçons $(44,3 \%)$ sont 2,38 fois plus nombreux que les filles (I8,6\%). En revanche, si l'on compare les pourcentages pour l'envoi des trois types de dédicaces, l'écart s'amoindrit: pour en force, les garçons ( $51,2 \%)$ sont seulement 1,69 fois plus nombreux que les filles (30,2\%); pour big up, les garçons ( $54 \%$ ) sont seulement 1,54 fois plus nombreux que les filles $(34,9 \%)$; pour représente, les garçons $(45,5 \%)$ sont seulement 1,25 fois plus nombreux que les filles $(36,3 \%)$. Ainsi, les filles, moins scripteures en général, sont plus présentes proportionnellement ; elles s'investissent plus dans la revendication territoriale à travers les dédicaces.

Tableau 2 : Répartition par sexe pour les messages incluant en force, big up et représente

\begin{tabular}{|c|c|c|c|c|}
\hline & enforce & bigup & représente & Totalité du corpus $^{18}$ \\
\hline Garçons & $5 \mathrm{I}, 2 \%(83)$ & $54 \%(34)$ & $45,5 \%(20)$ & $44,3 \%(16747)$ \\
\hline Filles & $30,2 \%(49)$ & $34,9 \%(22)$ & $36,3 \%(\mathrm{I} 6)$ & $18,6 \%(7015)$ \\
\hline Inconnu & $\mathrm{I} 8,5 \%(30)$ & $\mathrm{I} \mathrm{I}, \mathrm{I} \%(7)$ & $\mathrm{I} 8,2 \%(8)$ & $36,1 \%(13622)$ \\
\hline Total & $100 \%(162)$ & $100 \%(63)$ & $100 \%(44)$ & $100 \%(37776)$ \\
\hline
\end{tabular}

Le Tableau 3 présente la répartition par type de lieu concerné pour les trois types de dédicace. Une tendance se dégage des résultats à savoir : la proportion la plus importante de messages comprenant en force concerne un club sportif, celle comprenant big up concerne un établissement scolaire et celle comprenant représente concerne une ville ou un département. Une explication sémantique de ces résultats peut être envisagée : on soutient un club sportif (《en force »), on passe le bonjour aux amis du même établissement ( «big-up ») et on « représente » sa ville ou son département. ${ }^{19}$

I8 Rappel des chiffres donnés plus haut.

I9 La moyenne d'âge pour les scripteurs, en fonction du type de dédicace, a également été calculée. Elle est de 16,5 ans pour en force, 17,3 ans pour big-up et de 17,8 ans pour 
Tableau 3 : Répartition par type de lieu concerné pour les messages incluant en force, big up et représente

\begin{tabular}{|l|l|l|l|}
\hline & \multicolumn{1}{|c|}{ en force } & \multicolumn{1}{|c|}{ bigup } & \multicolumn{1}{c|}{ représente } \\
\hline $\begin{array}{l}\text { Établissement } \\
\text { scolaire }\end{array}$ & $8,0 \%(\mathrm{I} 3)$ & $34,9 \%(22)$ & $9, \mathrm{I} \%(4)$ \\
\hline Quartier & $4,9 \%(8)$ & $9,5 \%(6)$ & $\mathrm{I} 3,6 \%(6)$ \\
\hline Ville & $26,6 \%(43)$ & $23,8 \%(\mathrm{I} 5)$ & $34, \mathrm{I} \%(\mathrm{I} 5)$ \\
\hline Club sportif & $30,9 \%(50)$ & $\mathrm{I}, 6 \%(\mathrm{I})$ & $4,5 \%(2)$ \\
\hline Département & $22,2 \%(36)$ & $\mathrm{I} 7,5 \%(\mathrm{II})$ & $36,4 \%(\mathrm{I} 6)$ \\
\hline Région & $\mathrm{I}, 2 \%(2)$ & $0 \%(0)$ & $0 \%(0)$ \\
\hline Pays & $6,2 \%(\mathrm{I})$ & $\mathrm{I} 2,7 \%(8)$ & $2,3 \%(\mathrm{I})$ \\
\hline Total & $100 \%(\mathrm{I} 62)$ & $100 \%\left(6_{3}\right)$ & $100 \%(44)$ \\
\hline
\end{tabular}

Le Tableau 4 présente les résultats pour la provenance départementale des scripteurs en fonction des trois types de messages. Ils ont été classés par ordre décroissant du total des messages, les résultats présentés se limitent à 3 messages minimum. ${ }^{20}$ Les résultats obtenus corroborent les résultats présentés ci-dessous pour le corpus total, avec une prédominance pour les départements de l'Île-de-France et pour les grandes villes. Le département de la Seine-SaintDenis (93) est largement en tête, puis viennent des départements de la région Île-de-France (91, 94 et 77) puis des départements de grandes villes comme Marseille, Grenoble ou Lyon. N'ayant pas accès à la véritable identité des

représente. Cette faible différence n'est pas représentative mais, en analysant plusieurs corpus de ce type en diachronie, on pourrait certainement voir ici des germes d'une dynamique, d'un changement de mode dans les parlers jeunes.

20 Les départements desquels deux messages ont été envoyés sont : 03, I4, 56, 7I, 74, 8I, 83,84 et 89 . Les départements desquels I seul message a été envoyé sont : 06, 22, 25, $29,35,45,47,54,60,62,63,66,67,72,73,79,80,87$. Pour les autres départements, il n'y a pas d'occurrence. De par sa forte proportion de messages envoyés, le département I a été exclu de l'analyse car nous avons émis l'hypothèse que de nombreux jeunes qui avaient écrit I n'étaient pas originaires de l'Ain mais avaient noté le premier chiffre pour remplir la case, dans un but d'anonymat ou bien écrivaient de l'étranger, comme nous le verrons également lors de l'analyse des pseudos. 
scripteurs, il nous est impossible de vérifier cette hypothèse mais remarquons toutefois que ces départements correspondent à des territoires qui comportent de nombreux et célèbres (médiatiquement parlant) quartiers d'habitat social. ${ }^{21}$ Si l'on s'intéresse aux résultats en fonction du type de dédicace, remarquons que big up n'est pas fortement ancré territorialement mais que en force et représente le sont $(93,91$ et 94 pour en force; 93 et 77 pour représente).

Tableau 4 : Provenance départementale des scripteurs en fonction des messages incluant en force, big up et représente

\begin{tabular}{|c|c|c|c|c|c|}
\hline $\begin{array}{c}\mathrm{n}^{\circ} \mathrm{du} \\
\text { département }\end{array}$ & chef-lieu & en force & big up & représente & $\begin{array}{l}\text { Total des } \\
\text { messages }\end{array}$ \\
\hline 93 & Bobigny & $\mathrm{I} 2,3 \%(20)$ & $3,2 \%(2)$ & $13,6 \%(6)$ & 28 \\
\hline $9 \mathrm{I}$ & Évry & $9,3 \%(15)$ & $4,8 \%(3)$ & $2,3 \%(\mathrm{I})$ & Ig \\
\hline 94 & Créteil & $6,2 \%(10)$ & $4,8 \%(3)$ & $2,3 \%(\mathrm{I})$ & 14 \\
\hline 77 & Melun & $3,7 \%(6)$ & $6,3 \%(4)$ & $9, \mathrm{I} \%(4)$ & 14 \\
\hline I 3 & Marseille & $4,9 \%(8)$ & $4,8 \%(3)$ & $2,3 \%(\mathrm{I})$ & $I 2$ \\
\hline 38 & Grenoble & $4,3 \%(7)$ & $3,2 \%(2)$ & ०\% (०) & 9 \\
\hline 69 & Lyon & $3,7 \%(6)$ & $3,2 \%(2)$ & ०\% (०) & 8 \\
\hline 76 & Rouen & $3, \mathrm{I} \%(5)$ & $\mathrm{I}, 6 \%(\mathrm{I})$ & $4,5 \%(2)$ & 8 \\
\hline 95 & Pontoise & $3, \mathrm{I} \%(5)$ & $3,2 \%(2)$ & $2,3 \%(\mathrm{I})$ & 8 \\
\hline 42 & Saint-Etienne & $1,9 \%(3)$ & $6,3 \%(4)$ & $2,3 \%(\mathrm{I})$ & 8 \\
\hline 78 & Versailles & $3,1 \%(5)$ & $\mathrm{I}, 6 \%(\mathrm{I})$ & $2,3 \%(\mathrm{I})$ & 7 \\
\hline 34 & Montpellier & I,9\% (3) & ०\% (०) & $4,5 \%(2)$ & 5 \\
\hline $5 \mathrm{I}$ & $\begin{array}{l}\text { Châlons-en- } \\
\text { Champagne }\end{array}$ & $\mathrm{I}, 2 \%(2)$ & $4,8 \%(3)$ & ०\%(०) & 5 \\
\hline 59 & Lille & $\mathrm{I}, 9 \%(3)$ & $\mathrm{I}, 6 \%(\mathrm{I})$ & $2,3 \%(\mathrm{I})$ & 5 \\
\hline 44 & Nantes & $2,5 \%(4)$ & ०\% (०) & $2,3 \%(\mathrm{I})$ & 5 \\
\hline 75 & Paris & $2,5 \%(4)$ & ०\% (०) & $\circ \%(0)$ & 4 \\
\hline $3 \mathrm{I}$ & Toulouse & $0,6 \%(\mathrm{I})$ & $4,8 \%(3)$ & ०\%(०) & 4 \\
\hline
\end{tabular}

2I Par exemple : les 4000 à la Courneuve (93), les Tarterêts à Corbeil-Essonnes (9I), le Bois l'Abbé à Champigny-sur-Marne (94), les quartiers Nord de Marseille, Échirolles (banlieue de Grenoble), Villeurbanne (banlieue de Lyon), etc. 


\begin{tabular}{|l|l|l|l|l|l|}
\hline 92 & Nanterre & $0,6 \%(\mathrm{I})$ & $4,8 \%(3)$ & $\circ \%(\mathrm{O})$ & 4 \\
\hline $2 \mathrm{I}$ & Dijon & $\mathrm{I}, 2 \%(2)$ & $\mathrm{I}, 6 \%(\mathrm{I})$ & $\circ \%(\mathrm{O})$ & 3 \\
\hline 28 & Chartres & $0,6 \%(\mathrm{I})$ & $3,2 \%(2)$ & $\circ \%(\mathrm{O})$ & 3 \\
\hline 57 & Metz & $0 \%(\mathrm{O})$ & $3,2 \%(2)$ & $2,3 \%(\mathrm{I})$ & 3 \\
\hline 86 & Poitiers & $0,6 \%(\mathrm{I})$ & $\mathrm{I}, 6 \%(\mathrm{I})$ & $2,3 \%(\mathrm{I})$ & 3 \\
\hline
\end{tabular}

Le corpus datant de 2005 et comportant une semaine de messages, il est bien sûr impossible d'en tirer des conclusions générales, surtout sur une utilisation plus générale de ces trois types de dédicaces car, rappelons-le, nous sommes ici dans la situation spécifique de messages envoyés à la radio Skyrock. En revanche, nous pouvons toutefois constater que ce type de corpus nous permet de développer des pistes sur les profils de leurs utilisateurs et sur leur sémantisme. Nous avons souhaité comparer nos résultats avec le corpus des 68572 sms récoltés à Montpellier en 20 I par l'équipe de Rachel Panckhurst dans le cadre de sms 4 science $e^{22}$ et n'avons trouvé aucune occurrence de big up ni de représente (employé dans le sens d'une dédicace). En revanche, nous avons trouvé une occurrence de en force à savoir « Nutella en force », ce qui laisse déceler une certaine ironie, voire une reprise de la stigmatisation véhiculée en grande partie par les médias. Ce résultat ne fait que confirmer notre hypothèse d'un ancrage spécifique d'une partie des jeunes qui écrivent à Skyrock au niveau diatopique et diastratique. ${ }^{23}$

\section{Analyse du corpus sous l'angle des pseudos}

Le corpus de pseudos a été formé en enlevant 3625 messages où l'identifiant de l'auditeur était le numéro de téléphone et où aucune identification au niveau du profil sociologique n'a été demandée de la part de Skyrock.

22 < http://cental.fltr.ucl.ac.be/>. Comme l'initiative avait un ancrage universitaire, ce sont surtout des étudiants qui ont participé.

23 Pour une présentation de différents profils sociologiques de jeunes en fonction de leurs pratiques culturelles, voir Octobre (2014 : 137-I48). 
Les 34 I5I messages restants, soit 90,4\% du corpus initial, ont été envoyés plus de deux fois plus souvent par les garçons (20,6\%, resp. 49,0\%), tout en prenant compte du fait que le sexe de $30,4 \%$ des scripteurs n'a pas été indiqué (quasiment tous ont envoyé un sms).

Une situation similaire s'est produite pour l'indication du département d'où venaient les scripteurs : 92,1\% des jeunes envoyant un sms n'ont indiqué ni leur provenance ni leur âge, à la différence de moins de 0,05\% des scripteurs via la plateforme Internet. En revanche, cétait surtout dans cette catégorie que des données suspectes ont pu être trouvées, comme cela a été vu supra: le chiffre I a servi à la fois à indiquer la provenance de l'Ain (le logiciel de transfert de données a effacé les o devant les chiffres - c'est-à-dire que le département des Alpes-Maritimes est affiché 6 et non o6) et pour dire qu'on vient de l'étranger - ceci étant le cas également dans l'indication des chiffres des départements inexistants (p.ex. 96, 98 ou, le plus souvent, 99). La plateforme ne permettant pas d'ajouter un troisième chiffre, les auditeurs des DOM-TOMs n'ont pu donner que les deux premiers chiffres p.ex. le code de la Réunion étant le 974, l’auditeur n’a pu saisir que le 97 et écrire la dédicace dans le corps du message (SALUT C'EST BRUNO DE LA RéUNION, J'AIMERAIS DéDICACER MARIO - LET ME LOVE YOU à TOUS MES POTES QUI SONT EN MéTROPOLE à BORDEAUX ET à PARIS, LES CHINOIS, PHILO ... SIGNé LE MALBAR BLOND).

Les tricheries (ou plaisanteries) dans la catégorie de l'âge ont concerné l'indication I, 2 ou 3 ans ou, à l'extrémité, les âges de 75, 78, 99 ou même I27 ans (suite à la lecture du contenu des messages, nous allons catégoriser sous faux-profils au niveau de l'âge tout âge dépassant 55 ans (la catégorie 40-55 ans étant caractérisée par des messages écrits avec une orthographe moins réductrice au niveau du nombre des signes et par des demandes plus pragmatiques). Un âge « bizarre » n’a été mentionné que très rarement (0,0014\%). Notons encore que parfois, les colonnes âge et département ont été renversés (fille de is ans du 75 a écrit l'inverse : âge 75, département I5).

Le corpus trié est formé par I2 86I pseudonymes uniques (soit 37,7\% de la totalité des pseudos). Parmi les jeunes identifiés avec des pseudos, quatre ont envoyé plus de roo messages, la gratuité privilégiant le 
« flood ${ }^{24} \gg$. Ceci est en contraste avec les sms payants : parmi les jeunes identifiés, il y a seulement une petite dizaine de ceux qui ont envoyé plus de I5 sms (l'extrême étant une fille qui a envoyé 54 messages et une autre 33 messages, le reste ne dépassant pas $25 \mathrm{sms}$ ).

Si l'on divise les messages selon la façon dont ils sont parvenus à la station, les 160 messages envoyés via i-mode cachent 96 scripteurs dont 32 utilisent dans leur pseudonyme un chiffre qui n'a pas de motivation toponymique. Les i 8 autres en ont une (chiffre du département, le plus souvent le 59 - région lilloise et le $\mathrm{I} 3$ - marseillaise - $3 \mathrm{x}$ ) et un toponyme est inscrit en entier (Gwada). Le reste de notre corpus est constitué de messages envoyés via $S$ (sms) et via I (internet). Leur quantité est telle qu'elle ne nous permet pas d'analyser la totalité des pseudos. Nous laissons donc de côté les messages via I (23 179 messages de 8 I54 scripteurs) mais aussi les 9955 messages envoyés via $S$ qui sont inanalysables d'un point de vue du rapport du chiffre et du numéro de département car les scripteurs n'ont pas rempli la colonne du département pour dire d'où ils venaient. Les 852 messages restants ont été écrits par 348 scripteurs. Même si 220 d'entre eux ont intégré un chiffre dans leur pseudo, soit $63,2 \%$, seulement 48 ont un composant toponymique identifiable comme chiffre du département. Ce dernier est postposé au nom propre, souvent surnom (p.ex. OMAR69, MANU25, $\mathrm{KJ}_{2 \mathrm{I}}$ ), parfois avec la préposition DU / 2 (chiffre mis à la place de DE) interposée (p.ex. LOLODU77, MIKEDU92). Dans quelques cas, les jeunes utilisent le nom de la ville (p.ex. MANU 2 COLMAR, DAVID 2 SETE) ${ }^{25}$ ou le nom du pays d'origine (p.ex. MISSALGERIE), etc ${ }^{26}$.

24 Nous considérons comme flood la répétition de messages comportant exactement le même contenu ou un contenu légèrement différent. Le flood touche le plus souvent les messages envoyés via l'Internet qui sont envoyés gratuitement.

25 Plus souvent, on voit l'adjectif nominalisé comme, par exemple LE NANTAIS ou L'AUXERROISE.

26 Ajoutons que les jeunes, notamment les garçons, se donnent comme pseudos des variantes du type 3I ENFORCE, 77 REPRESENTE, 80 STYLE (ces relevés proviennent du corpus des messages provenant d'Internet, où les pseudos restent inexploités statistiquement pour le moment). 
Nous remarquons, non sans surprise, que parmi les pseudonymes avec un chiffre qui n'est pas celui du département ${ }^{27}$, une catégorie de chiffre à trois numéros peut être décelée. Dans de nombreux cas, il s'agit d'une stratégie de la part des jeunes scripteurs pour spécifier la commune du département : par exemple, on peut supposer que $\mathrm{COCO}_{44 \mathrm{I}}$ qui vient du 78 habite à Nainville les Roches (7844I).

\section{Le poids des villes et des départements}

L'analyse du corpus a mis au jour le poids des villes et des départements dans une forme de revendication territoriale telle qu'elle est manifestée par les jeunes scripteurs de la radio Skyrock. La question des villes mériterait d'être analysée plus amplement du point de vue de la présence d'argotoponymes (Podhorná 2002) comme VLB (Villiers-le-Bel ; sigle), Auber (Aubervilliers ; apocope), Panam / Paname (Paris ; vieil argot) ou même Boboch / Boboche ${ }^{28}$ (Bobigny; apocope et resuffixation argotique). La question identitaire du département n'est pas nouvelle. Les codes à deux chiffres (voire trois pour les DOMs) dans les pseudos font référence, de façon univoque, au département en question dans le système administratif français, à la différence du tchèque (David 2009 : 48) où les deux chiffres indiquent toujours l'âge du scripteur ${ }^{29}$.

27 Il s'agit soit d'un chiffre qui permet de dissocier les scripteurs du même nom ou surnom (AMELIE2), soit d'un numéro qui ressemble au département mais qui ne coïncide pas avec celui indiqué dans la case « domicile ». Il se peut aussi que les jeunes, notamment ceux âgés de plus de I 8 ans y aient indiqué le département dans lequel ils étudient actuellement et que leur pseudo cache l'information de leur provenance. Il a été relevé $\mathrm{I} 4$ fois dans le corpus comme par exemple ici : «BIZOU A TOU CEU KI GALERE DAN LE 93 A BOBOCH » (fille, I4 ans, 93)

29 Ce qui n'est qu'un fait rarissime dans notre corpus (dans ce cas, on voit quelquefois

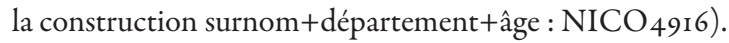


Si l'on retrace le phénomène de revendication symbolique à travers les chansons de rap (Podhorná-Polická 20I2), il a d'abord été observé pour le département de la Seine-Saint-Denis (le célèbre « neuf-trois », 9.3) puis, par attraction, il s'est répandu aux autres départements commençant par quatre-vingt-dix (91, 92, 94 et 95) puis aux départements de l'île-de-France $(77,78,75)$, et enfin aux départements et territoires français en général (par exemple, le « neuf-sept-quatre » pour la Réunion). Sur Skyrock, les animateurs demandent très souvent (voire toujours) aux auditeurs d'où ils appellent, ce qui a certainement contribué largement à la diffusion de cette forme. Comme l'une d'entre nous a pu le constater pour les rappeurs: « la forte convergence des [scripteurs] vers la ( $\mathrm{p}$ )référence macro-toponymique s'explique, à destination de l'intérieur, par son universalité, laquelle permet d'encadrer même les [scripteurs] habitant les villes ou quartiers moins « réputés » ou bien les collectifs issus de différentes villes et qui instaure en même temps un repère identitaire pour un éventail plus large d'auditeurs. Elle s'explique, à destination de l'extérieur, par deux logiques, à la fois concomitantes et antagonistes : a) la contiguïté solidaire avec les autres départements et b) la fierté compétitive vis-à-vis des rappeurs d'autres départements que l'on peut qualifier, à l'instar de Vicherat, d' « orgueil territorial » (Vicherat 200I : 42). » (Podhorná-Polická 2012 : 89). À l'époque de la discussion sur la réforme territoriale ${ }^{30}$, cette étude montre - du moins pour l'analyse de ce corpus - une quasi-inexistence de la région au niveau de l'appropriation territoriale.

Sur les réactions face au projet de réforme territoriale, voir deux articles publiés dans le quotidien régional Le Parisien : « La Seine-Saint-Denis va-t-elle disparaître » (<http://www.leparisien.fr/espace-premium/seine-saint-denis-93/la-seine-saintdenis-va-t-elle-disparaitre-I7-OI-20I4-350I56I.php>) et «Une part d'identité qui s'en va $\gg$ (<http://www.leparisien.fr/espace-premium/seine-saint-denis-93/ une-part-d-identite-qui-s-en-va-I7-OI-20I4-350I563.php >), tous deux publiés en ligne le 17 janvier 2014 . 


\section{Pour aller plus loin}

Le corpus de sms envoyés à la radio Skyrock s'avère être une riche mine pour les analyses sur l'appropriation territoriale dont nous avons essayé de proposer un témoignage parcellaire, mais pas seulement. En effet, il semble propice pour l'observation des innovations lexicales, des analyses conversationnelles auditeur-scripteur $v$ s animateur-lecteur, etc. Étant donné que nous avons pu constater quelques universaux dans la formation des pseudos en français et en tchèque (voir le corpus de David 2009), nous pouvons également espérer une analyse contrastive. La perspective pourrait également s'élargir considérablement, notamment au niveau d'une analyse diachronique, si les circonstances nous le permettent.

\section{Références}

Anis Jacques, 200I, Parlez-vous texto ?, guide des nouveaux langages du réseau, Paris, Le cherche midi.

Bastian Sabine, Bulot Thierry \& Burr Élisabeth (Eds), 2009, Sociolinguistique urbaine et développement durable urbain (Enjeux et pratiques dans les sociétés francophones et non francophones), Munich, Martin Meidenbauer Verlag.

Bernicot Josie, Goumi Antonine, Bert-Erboul. Alain \& Volckaert-Legrier Olga, 2014, « How do skilled and less-skilled spellers write text messages ? A longitudinal study of sixth and seventh graders », in Journal of Computer Assisted Learning, $<$ doi: I0.IIII/jcal.I2064>.

Boyer Henri \& Lochard Guy, 1998, Scènes de télévision en banlieues, 1950-1984, Paris, L'Harmattan.

Bulot Thierry, 20II, « Sociolinguistique urbaine, Linguistic Landscape Studies et scripturalité : entre convergence(s) et divergence(s) », in Cabiers de Linguistique, 37/1, 5-15.

Cougnon Amélie \& Ledegen Gudrun, 2010, « C'est écrire comme je parle. Une étude comparatiste de variétés de français dans l' 'écrit sms' », in Abecassis Michael \& Ledegen Gudrun (Éds), Les voix des Français, Oxford, Peter Lang (Modern French Identities, vol. 2), 39-57. 
David Jaroslav, 2009, « Nicky v komunikaci na www chatu », in Jandová Eva et al.: Čeśtina na www chatu, Ostrava, Ostravská univerzita, 43-84.

Fairon Cédrick, 2010, « 'Langage sms' et convergence numérique », in Langue et cité, 20, 4-5.

Fairon Cédrick, Klein Jean-René \& Paumier Sébastien, 2006, Le langage sms, étude d'un corpus informatisé à partir de l'enquête « Faites don de vos sms à la science», Louvain-la-Neuve, Presses universitaires de Louvain.

Fiévet Anne-Caroline, 2007, « Le français contemporain des cités dans les émissions des radios jeunes $\gg$, in Adolescence, 59, I25-131.

Fiévet Anne-Caroline, 2008a, Peut-on parler d'un argot des jeunes ?: analyse du lexique argotique employélors d'émissions de libre antenne sur Skyrock, Fun Radio et NRJ, Thèse de doctorat, Université Paris Descartes.

Fiévet Anne-Caroline, 2008b, «Libre antenne : l'argot des jeunes », in Médiamorphoses, 23, 7I-74.

Glevarec Hervé, 2005, Libre antenne, La réception de la radio par les adolescents, Paris, Armand Colin.

Glevarec Hervé \& Pinet Michel, 2009, La radio et ses publics, Paris, éditions Irma.

Jamin Michaël, 2005, Sociolinguistic variation in the Paris suburbs, Thèse de Doctorat, Université of Kent at Canterbury.

Kokoreff Michel, 2003, La force des quartiers : de la délinquance à l'engagement politique, Paris, Payot.

Ledegen Gudrun \& Simonin Jacky, 2010, « Médias et pratiques langagières à La Réunion : accélérateur sociolinguistique et diglossie en sourdine », in Glottopol, I4, IO4-II6.

Lepoutre David, 1997, Cour de banlieue- codes, rites et langage, Paris, Odile Jacob.

Liogier Estelle, 2006, Langue « du quartier » et français «standard 》 dansle répertoire verbal d'adolescents de cité, Thèse de Doctorat, Université Paris Descartes.

Melliani Fabienne, 2000, La langue du quartier, appropriation de l'espace et identités urbaines chez des jeunes issus de limmigration maghrébine en banlieue rouennaise, Paris, l'Harmattan.

Octobre Sylvie, 2014, Deux pouces et des neurones, Les cultures juvéniles de l'ère médiatique à l'ère numérique, Paris, Ministère de la Culture et de la Communication, Département des études, de la prospective et des statistiques (DEPS).

Panckhurst Rachel, 1997, « La communication 'médiatisée' par ordinateur ou la communication 'médiée' par ordinateur ? », in Terminologies nouvelles, 17, 56-58.

Panckhurst Rachel, Détrie Catherine, Lopez Cédric, Moïse Claudine, Roche Mathieu \& Verine Bertrand, 20I3, « Sud4science, de l'acquisition d'un grand corpus de sms en français à l'analyse de l'écriture sms », in Épistémè-revue internationale de sciences, 9 , $107-138$. 
Pasquier Dominique, 1999, La Culture des sentiments, l'expérience télévisuelle des adolescents, Paris, Éditions de la Maison des sciences de l'homme.

Podhorná Alena, 2002, Toponymie et argots : les argotoponymes en français contemporain des cités (L'exemple de la Cité des 4000 à La Courneuve, Seine-Saint-Denis), Mémoire de D.E.A., Paris, Université René Descartes.

Podhorná-Polická Alena, 2012, « La circulation des innovations lexicales dans un espace territorialement circonscrit, le cas des jeunes dits ' des quartiers' et du rap dans le Val-de-Marne », in Bulot Thierry et Feussi Valentin, Normes, Urbanités et émergences plurilingues, Paris, L'Harmattan, 85-104.

Rui Sandrine, 1995, « La foule sentimentale. Récit amoureux, média et réflexivité », in Réseaux, 70, 105-119.

Sauvadet Thomas, 2004, "'Jeunes de la cité' et contrôle du territoire : le cas d'une cité de la banlieue parisienne », in Hérodote, I3, 2/2004, II3-I33.

Sow Papa Alioune, 2010, « Normes et discursivités, le parler jeune dans les émissions radiophoniques », in Glottopol, $14,37-48$.

Trimaille Cyril, 2004, « Étude de parlers de jeunes en France : éléments pour un état des lieux », in Cabiers de sociolinguistique, 9, 99-132.

Vašková Petra, 20II, Le lexique argotique sur Skyrock : analyse des néologismes en synchronie dynamique (2003 et 2008), Mémoire de master, Masarykova univerzita v Brně.

Vicherat Mathias, 200I, Pour une analyse textuelle du rap français, Paris, L'Harmattan. 


\section{La particule négative ne dans les français d'A frique et de l'Océan Indien : convergences et divergences}

La faible réalisation du ne de négation est souvent présentée comme une variable classique dans les études de sociolinguistique, comme exemple d'une variation généralisée, non localisée :

Dans Si jaurais su, je serais pas venu, on peut relever deux écarts grammaticaux : le conditionnel après si et la négation réduite à pas. Cela s’observe partout : ces deux écarts ne sont pas des régionalismes. (Tuaillon $1983: 227$ )

caractérisant tous les locuteurs :

ne deletion/retention [characterises] all speakers. It is not just 'working-class' or 'uneducated' speakers who drop ne in sentences such as the above but, given an appropriate context (e.g. an informal family situation), even the most 'educated' speakers will use variants without ne. (Durand $1993: 262$ )

van Compernolle \& Williams (2009) indiquent ainsi que de faibles fréquences de maintien de ne - ils mentionnent le seuil de « moins de 20\% » (2009: 419) - s'attestent ainsi pour le français informel en France (Armstrong 2002 ; Ashby 200I ; Coveney 1998 ; Hansen et Malderez 2004), et que l'omission devient presque catégorique au Canada (Poplack et St-Amand 2007; Sankoff et Vincent 1977) et en Suisse (seulement $2,5 \%$ de maintien de ne dans les études de Fonseca-Greber 2000, 2007). À l'inverse, Queffélec fait mention, pour le terrain africain, d'un maintien plus élevé :

le maintien très fréquent (statistiquement largement supérieur à ce qu’on observe en français oral européen) de l'adverbe ne comme signe de la négation verbale : 
même en situation informelle, cet indice négatif clitique reste présent dans le discours parlé africain, là où il a largement disparu en français hexagonal. (Queffélec 2008: 73)

Notre étude de la particule négative ne dans les français d'Afrique et de l'Océan Indien, à travers le cas de La Réunion, tentera de cerner les influences possibles du contact linguistique : différents terrains africains et le terrain réunionnais au sein du programme PFC sont confrontés aux pratiques innovantes des SMS afin de cerner les convergences et divergences. La comparaison de ces terrains entre eux, avec le terrain métropolitain et celui de la francophonie en général à travers la base PFC révèlera la nécessité d'un traitement pluriel de la particule ne. Cette comparaison s'inscrit pleinement dans la définition globale et non-hiérarchique de la francophonie dessinée par Carol Sanders, dans le but « de voir comment une langue - le français - évolue dans des conditions et des situations de contact différentes » (Sanders 2004: 8). La comparaison des données de plusieurs variétés du français, pour y explorer des différences ou en souligner les ressemblances, est menée ici en dehors de toute idée de « hiérarchie dominée par le 'bon' français de France » (Sanders 2004: 8), comme l'auteure l'identifiait encore dans l'étude de Valdman, Le français hors de France (1979) ou les deux tomes de Robillard et Beniamino, Le français dans l'espace francophone (1993-1996).

Notre article s'organise comme suit : après avoir brièvement rappelé les résultats de quelques études antérieures (Section I), nous envisagerons deux terrains où le français se trouve en contact avec une voire plusieurs langues. Nous étudierons tout d'abord des données de parole spontanée obtenues dans 6 pays de la zone sub-saharienne (Section 2), et les données recueillies dans l'Île de la Réunion à partir d'un corpus de parole spontanée et d'un corpus de SMS (Section 3). Nous conclurons (Section 4) en mettant en exergue le rôle crucial joué par la prosodie et la syntaxe. 


\section{Absence de la particule : données européennes}

S'il semble qu'au $17^{\mathrm{e}}$ siècle, la distinction entre présence et absence de la particule ne était surtout d'ordre stylistique ${ }^{1}$ (Kawaguchi 2009), tel n'est plus le cas au $20^{\mathrm{e}}$ siècle où l'usage tend de plus en plus vers des phrases négatives dotées du seul adverbe pas. Depuis les années i950, le développement des corpus a permis de mettre en évidence le faible taux de réalisation de la particule dans l'usage quotidien.

Tableau I : Maintien de ne dans différents corpus

\begin{tabular}{|c|c|c|c|}
\hline Auteur & Année & Lieu & Maintien de $n e$ \\
\hline Pohl I968 & Début des années so & Belgique/ France & $61,9 \%$ \\
\hline Sankoff et Vincent i 980 & 1971 & Montréal & $0,5 \%$ \\
\hline Ashby 1976 & $1967-68$ & Paris & $55,8 \%$ \\
\hline Diller I 983 & I975 & $\begin{array}{l}\text { Béarn } \\
\text { Classe supérieure } \\
\text { Classe populaire }\end{array}$ & $\begin{array}{l}58 \% \\
78 \%\end{array}$ \\
\hline Ashby I 98 I & 1976 & Tours & $58 \%$ \\
\hline Coveney I 996 & 1980 & Somme & I $8,8 \%$ \\
\hline Moreau I 986 & $1982 / 83$ & Belgique & $50,2 \%$ \\
\hline Pooley I 996 & 1983 & Roubaix & $7 \%$ \\
\hline Armstrong 2002 & 1990 & Lorraine & $\mathrm{I}, 8 \%$ \\
\hline Pooley I 996 & 1995 & $\begin{array}{l}\text { Rouge-Barres } \\
\text { (Nord) }\end{array}$ & $\mathrm{I} \%$ \\
\hline Ashby 200 I & I995 & Tours & I $5,7 \%$ \\
\hline $\begin{array}{l}\text { Hansen et Malderez } \\
2004\end{array}$ & I $992-93$ & Paris & $7 \%$ \\
\hline
\end{tabular}

I Le grammairien Ménage s’oppose à Vaugelas en ces termes : « Monsieur de Vaugelas veut qu' il soit mieux de dire, Ont-ilspas fait? sans négative, que N'ont-ilspas fait? avec que la négative. Je ne suis pas de son avis. N'ont-ils pas fait me semble plus élégant » (cité par Kawaguchi 2009: 206). 
Les écarts de pourcentages dont fait état le Tableau I ${ }^{2}$ proviennent partiellement d'une certaine disparité géographique (Montréal vs Belgique) mais également de la disparité des corpus étudiés : corpus radiophonique dans Moreau (1986), conversations informelles dans Pooley (1996). Seules les données de Ashby (198I) et (200I) construites comme une étude en temps réel autorisent une véritable comparaison : les deux corpus ont été construits dans la même ville, selon le même protocole et reprennent en partie les mêmes témoins. Ashby (200I) conclut ainsi à un changement en temps réel : la particule ne serait en perte de vitesse aussi bien chez les hommes que chez les femmes, dans toutes les tranches sociales toutes classes d'âge confondues.

Tableau 2 : Évolution, facteurs de variation dans le maintien de ne (Ashby 200I)

\begin{tabular}{|l|c|c|c|}
\hline \multicolumn{1}{|c|}{ Locuteurs } & I976 & I995 & Différence \\
\hline I 4-22 ans & I9 & I 4 & -5 \\
\hline 5 I-64 ans & 52 & 25 & -27 \\
\hline Femmes & 30 & I7 & -13 \\
\hline Hommes & 42 & 20 & -22 \\
\hline Classe supérieure & 45 & 26 & -19 \\
\hline Classe moyenne & 47 & I7 & -30 \\
\hline Classe populaire & I 5 & 9 & -6 \\
\hline Total & 37 & I 8 & -19 \\
\hline
\end{tabular}

L'ensemble des études sur le comportement de la variable ne s'accorde sur sa faible présence dans les corpus étudiés soumise à une variation due au registre, à la classe socio-économique et à l'âge. Les facteurs purement linguistiques qui favorisent la chute constituent également un autre point de convergence. Parmi les plus cités, on trouve la nature de l'adverbe négatif, la nature du sujet et la fréquence de l'expression. Ainsi pas est de loin l'adverbe le plus susceptible d'induire l'absence, ce dont témoigne par

2 Tableau repris en grande partie de Armstrong et Smith (2002) 
exemple le corpus de Coveney (1996) avec la distribution suivante : pas $(83,6 \%)>$ rien $>$ jamais $>$ personne $(66,7 \%)$. Le poids prosodique et sémantique du sujet contribue également au maintien ou non de la particule : un sujet lexical protège la particule qui disparait plus facilement dans une suite de clitiques. Ce comportement s'accentue encore lorsqu'il s'agit d'expressions fréquentes qui sont les plus prônes à perdre la particule $n e$. Moreau (1986) et Ashby (198I) par exemple s’accordent à présenter les groupes suivants comme des séquences préformées : c'est pas, je sais pas, il faut pas, il y a pas. Les données que nous pouvons extraire de la base PFC ${ }^{3}$ (Phonologie du Français contemporain) (Durand, Laks et Lyche 2009) se conforment largement aux études antérieures, ce que nous illustrons à l'aide des résultats recueillis auprès des I 4 locuteurs de l'enquête menée dans le milieu de la haute bourgeoisie parisienne. Selon Ashby (200I), les locuteurs appartenant à la classe supérieure exhibent un taux relativement élevé de maintien de la particule (voir Tableau 2). Le niveau social de nos locuteurs nous inciterait à envisager l'hypothèse d'un système conservateur où la particule est relativement bien ancrée, hypothèse infirmée par les données du Tableau 3.

Tableau 3 : Présence de ne dans l'enquête PFC de la haute bourgeoisie parisienne

\begin{tabular}{|c|c|c|}
\hline \multirow{2}{*}{ Paris } & \multicolumn{2}{|c|}{ Maintien de la particule dans ne ... pas } \\
\hline & Entretien guidé & Entretien libre \\
\hline $20-39$ & $6.6 \%(8 /$ I 21$)$ & $9.6 \%(\mathrm{I} 4 / \mathrm{I} 46)$ \\
\hline $40-59$ & $13.2 \%(15 / 114)$ & $\mathrm{I} .5 \%(\mathrm{I} / 68)$ \\
\hline $60+$ & $34.3 \%(12 / 35)$ & $17.9 \%(12 / 67)$ \\
\hline Total & $13 \%(35 / 270)$ & $9.6 \%(27 / 28 I)$ \\
\hline
\end{tabular}

Rappelons que cette base de données comprend 400 locuteurs du monde francophone tous enregistrés à partir d'un protocole unique (Durand et Lyche 2013). Tous les locuteurs lisent la même liste de mots et le même texte, tous les locuteurs participent à un entretien guidé et une conversation libre. 
Le Tableau 3 met en évidence massive de la particule tout en soulignant l'importance du registre et de l'âge. Les locuteurs âgés utilisent la particule 5 fois plus souvent que la plus jeune génération lors de l'entretien guidé et deux fois plus souvent dans l'entretien guidé que dans la conversation libre. L'ensemble des études de corpus mentionnées convergent ainsi vers un très faible taux de réalisation de la particule et les résultats semblent suffisamment robustes pour leur opposer les résultats obtenus à partir des enquêtes menées sur le terrain africain et sur le terrain réunionnais, dans des zones où le français côtoie quotidiennement des langues typologiquement plus ou moins éloignées.

\section{L’Afrique}

Le français est majoritairement « langue seconde » sur le terrain africain. Ce terme, largement utilisé pour caractériser la situation africaine, couvre plus qu'une réalité linguistique (le français y est rarement acquis comme langue maternelle), il couvre également une réalité psycholinguistique : le français dans ces régions se distingue des autres langues étrangères en ce que « [il] joue dans leur [les membres de la communauté] développement psychologique, cognitif et informatif, conjointement avec une ou plusieurs langues, un rôle privilégié » (Cuq 199I : 139). Le français est traditionnellement appris lors de la scolarisation, c'est la langue officielle des pays concernés, c'est la langue de tout l'enseignement et de l'administration. Dans des sociétés toujours plurilingues, le français est parfois troisième, voire quatrième langue, mais c'est toujours la langue à laquelle l'enfant est confronté lorsqu'il est scolarisé.

Nos données africaines pour cette étude proviennent de 6 enquêtes PFC menées selon le protocole PFC strictement appliqué (Durand et Lyche 2013) dans 6 capitales de pays de Afrique de l'ouest tous officiellement francophones. 
Tableau 4 : Enquêtes PFC en Afrique sub-saharienne

\begin{tabular}{|l|l|c|}
\hline \multicolumn{1}{|c|}{ Pays } & \multicolumn{1}{c|}{ Enquête } & Nombre de locuteurs \\
\hline Côte d'Ivoire & Abidjan & I 4 \\
\hline Burkina Faso & Ouagadougou & I 2 \\
\hline Mali & Bamako & I 3 \\
\hline Cameroun & Yaoundé & 6 \\
\hline Centrafrique & Bangui & I 3 \\
\hline Sénégal & Dakar & I 2 \\
\hline
\end{tabular}

Dans les 6 pays, le degré de pratique et d'appropriation du français fait état de divergences profondes. La Côte d'Ivoire par exemple s'affirme sans nul doute comme le pays le plus francophone avec ses 4 grandes variétés de français qu'il y est coutume de relever (Boutin et Turcsan 2009) : le français académique, le français ivoirien, le français populaire ivoirien et le nouchi. Le français y est largement parlé par la population, il peut même être, en milieu urbain, la langue première des locuteurs. De l'autre côté du prisme se trouve le français malien ou centrafricain qui bien que langue officielle et langue de l'enseignement voit sa place dans la sphère linguistique fragilisée par l'existence de langues nationales à fonction véhiculaire (le bambara pour le Mali, le sango pour la Centrafrique). Dans ces deux pays, le français est très peu parlé par la population et n'intervient que rarement dans les interactions quotidiennes. Comme dans le reste de l'Afrique, tous ces terrains d'enquête se caractérisent par un plurilinguisme étendu où de nombreuses langues typologiquement très variées coexistent et sont régulièrement pratiquées par les locuteurs. Ce plurilinguisme étendu colore le français de traits issus de la langue locale de telle sorte que chaque variété subit une fragmentation et on parlera par exemple de français malien-bambara, français malien-songhay ${ }^{4}$. Au-delà de cet éclatement du nombre de variétés dû à l'influence d'une Li, les français africains ont en partage un ensemble de traits qui les distinguent des variétés hexagonales ou européennes. En 
effet, bien que chaque terrain exhibe sa panoplie de particularités phonologiques, l'analyse phonologique de chaque point d'enquête (par ex. Boutin et Turcsan 2009, Lyche et Skattum 2012, Bordal 2012, Boutin et al. 2012) permet de dégager quelques traits panafricains comme l'absence de véritable schwa, la faiblesse de la rhotique, un système de liaison très appauvri. Dans ce contexte, il semble légitime de s'interroger comme le fait Queffélec sur le rôle de la particule : Est-ce que l'absence de la particule négative constitue également un élément fédérateur dans les français d'Afrique? Afin de répondre à cette question, nous opposons les deux terrains que nous avons présentés comme le plus et le moins francophones soit respectivement Abidjan et Bamako.

Tableau 5 : Présence de ne dans la suite ne ... pas

\begin{tabular}{|c|l|l|l|l|l|}
\hline Abidjan & \multicolumn{1}{|c|}{ guidé } & \multicolumn{1}{c|}{ libre } & \multicolumn{1}{c|}{ Bamako } & \multicolumn{1}{c|}{ guidé } & \multicolumn{1}{c|}{ libre } \\
\hline $20-39$ & $\begin{array}{l}36 . \mathrm{I} \% \\
(26 / 7 \mathrm{I})\end{array}$ & $\begin{array}{l}36.6 \% \\
(26 / 7 \mathrm{I})\end{array}$ & $20-39$ & $\begin{array}{l}67 \% \\
(6 \mathrm{I} / 9 \mathrm{I})\end{array}$ & $\begin{array}{l}40.3 \% \\
(29 / 72)\end{array}$ \\
\hline $40-59$ & $\begin{array}{l}42.6 \% \\
(26 / 6 \mathrm{I})\end{array}$ & $\begin{array}{l}24,4 \% \\
(66 / 270)\end{array}$ & $40-59$ & $\begin{array}{l}53.8 \% \\
(42 / 78)\end{array}$ & $\begin{array}{l}44.3 \% \\
(3 \mathrm{I} / 70)\end{array}$ \\
\hline & & $60+$ & $\begin{array}{l}75.5 \% \\
(40 / 53)\end{array}$ & $\begin{array}{l}83.3 \% \\
(40 / 48)\end{array}$ \\
\hline Total & $39.3 \%$ & $\begin{array}{l}26.9 \% \\
(92 / 34 \mathrm{I})\end{array}$ & & $\begin{array}{l}64.4 \% \\
(\mathrm{I} 43 / 222)\end{array}$ & $\begin{array}{l}52.6 \% \\
(\mathrm{I} 00 / \mathrm{I} 90)\end{array}$ \\
\hline
\end{tabular}

Si dans les deux points d'enquête, la particule semble solidement établie, nous observons néanmoins un écart relativement important entre les deux terrains avec une plus forte tendance au maintien à Bamako. Cette tendance opère particulièrement dans la tranche d'âge $60+$ pour laquelle nous ne disposons malheureusement pas de locuteur à Abidjan. Il pourrait s'agir d'un effet dû à l'âge, ou refléter un niveau d'instruction plus élevé et une pratique plus importante de l'écrit ${ }^{5}$. Nous ferons l'hypothèse que 
la vernacularistion du français favorise la chute de la particule négative à Abidjan sans qu'elle atteigne pour autant des taux proches des données hexagonales. En effet, la moyenne des taux de maintien à Abidjan et Bamako s'avère largement supérieure aux taux de maintien à Paris et la comparaison des résultats fournit une différence statistiquement significative.

Tableau 6 : Maintien de ne (Paris vs Abidjan et Bamako)

\begin{tabular}{|c|c|c|}
\hline $\begin{array}{c}\text { Comparaison Paris/ } \\
\text { Abidjan+Bamako }\end{array}$ & guidé & libre \\
\hline valeurp & $6,426_{13} E-3 I$ & $7,38299 E-24$ \\
\hline
\end{tabular}

Sur la base du Tableau 6, nous pouvons conclure dans un premier temps que la présence remarquable de la particule constitue une exception africaine. Ces résultats viennent confirmer l'observation de Queffélec, mais il convient maintenant d'analyser de plus près les données afin de porter un éclairage sur les facteurs qui sont les plus susceptibles d'entraîner la chute de ne. Parmi les facteurs purement linguistiques, toutes les études antérieures effectuées en Europe relèvent l'importance des séquences dites préformées (Moreau 1986) ainsi que celle de la nature du sujet. Nous comparons donc dans les tableaux ci-dessous les séquences préformées les plus communément dépourvues de particule négative, soit (il) fautpas, c'estpas, (il) y a pas. Nous comparons les résultats des 6 enquêtes africaines à ceux de toute la base PFC.

Tableau 7 : (il) (ne) faut pas en Afrique et la base PFC

\begin{tabular}{|l|l|c|l|l|}
\hline & Afrique & \multicolumn{1}{|c|}{$\%$} & \multicolumn{1}{|c|}{ Reste base } & $\%$ \\
\hline faut pas & $\mathrm{I} 3 / 4 \mathrm{I}$ & $3 \mathrm{I}, 7$ & $68 / \mathrm{I} 75$ & 38,8 \\
\hline il faut pas & $25 / 4 \mathrm{I}$ & 60,9 & $99 / \mathrm{I} 75$ & 56,5 \\
\hline ilne faut pas & $3 / 4 \mathrm{I}$ & 7,3 & $8 / 175$ & 4,5 \\
\hline & & & Valeurp & 0,58 \\
\hline
\end{tabular}


Avec uniquement 7,3\% de réalisations de la particule, il semble justifié de postuler l'émergence d'une séquence préformée faut pas/il faut pas en Afrique également, même si le phénomène reste en-deçà de ce que l'on observe dans le reste de la base. L'écart s'élargit encore avec la séquence c'est pas.

Tableau $8: c e\left(n^{\prime}\right)$ est pas en Afrique et la base PFC

\begin{tabular}{|l|l|l|l|l|}
\hline & Afrique & \multicolumn{1}{|c|}{$\%$} & \multicolumn{1}{|c|}{ Reste base } & \multicolumn{1}{|c|}{$\%$} \\
\hline c'est pas & $320 / 355$ & 90,2 & 1 $805 / 1842$ & 98 \\
\hline cen'estpas & $35 / 355$ & 9,8 & $37 / 1842$ & 2 \\
\hline & & & Valeurp & $2,80134 E-14$ \\
\hline
\end{tabular}

L’absence de particule dans la séquence c'est pas fédère de façon quasi catégorique toute la base PFC. Le taux de rétention de la particule quoiqu' inférieur à Io\% en Afrique, reste néanmoins significativement élevé et témoigne d'une tendance robuste au maintien. Cette tendance se renforce encore dans il n'y a pas.

Tableau 9 : (il) (n')y a pas en Afrique et la base PFC

\begin{tabular}{|l|l|c|l|l|}
\hline & Afrique & $\%$ & \multicolumn{1}{|c|}{ Reste base } & \multicolumn{1}{|c|}{$\%$} \\
\hline y a pas & $20 / 157$ & $\mathrm{I} 2,7$ & $45 / 507$ & 8,8 \\
\hline ilyapas & $\mathrm{I} 19 / \mathrm{I} 57$ & 75,8 & $43 \mathrm{I} / 507$ & 85 \\
\hline iln'y a pas & $\mathrm{I} 8 / \mathrm{I} 57$ & $\mathrm{II}, 4$ & $3 \mathrm{I} / 507$ & $6, \mathrm{I}$ \\
\hline & & & Valeurp & 0,021 \\
\hline
\end{tabular}

La séquence il n’y a pas avec ses II, $4 \%$ de réalisations se caractérise comme la plus robuste des trois séquences étudiées et s'oppose largement au reste de la base PFC. Dans les trois tableaux cependant, il convient de noter un taux relativement faible de présence de la particule qui ne dépasse pas les $12 \%$. Nous pouvons alors conclure à une tendance en Afrique à la mise en place de séquences préformées d'où la particule négative se voit éliminée. 
Parmi les séquences préformées envisagées par Moreau (1986), nous avons choisi je sais pas et je pense pas pour leur fréquence toute relative dans le corpus ${ }^{6}$.

Tableau io : je (ne) sais pas en Afrique et la base PFC

\begin{tabular}{|l|l|c|l|l|}
\hline & Afrique & $\%$ & \multicolumn{1}{|c|}{ Reste base } & \multicolumn{1}{|c|}{$\%$} \\
\hline je saispas & $\mathrm{I} 49 / \mathrm{I} 95$ & 76,4 & $\mathrm{I} 520 / \mathrm{I} 644$ & 92,4 \\
\hline jene saispas & $46 / \mathrm{I} 95$ & 23,5 & $\mathrm{I} 24 / \mathrm{I} 644$ & 7,5 \\
\hline & & & Valeurp & 2,5769 IE-I3 \\
\hline
\end{tabular}

Tableau ir : je (ne) pense pas en Afrique et la base PFC

\begin{tabular}{|l|l|c|l|l|}
\hline & \multicolumn{1}{|c|}{ Afrique } & $\%$ & \multicolumn{1}{|c|}{ Reste base } & \multicolumn{1}{|c|}{$\%$} \\
\hline jepense pas & $6 / \mathrm{I} 2$ & 50 & $98 / 104$ & 94,2 \\
\hline jenepensepas & $6 / \mathrm{I} 2$ & 50 & $6 / 104$ & 5,8 \\
\hline & & & Valeurp & I,8998IE-o6 \\
\hline
\end{tabular}

L'écart entre l'Afrique et le reste de la base s'accentue profondément dans les Tableaux io et II avec une stabilité remarquable de la particule dans le corpus africain alors que les chiffres dans le reste de la base évoluent peu. Si les locutions impersonnelles des Tableaux 7,8 et 9 se figent relativement aisément sans la particule, tel n'est pas le cas en ro ou ir où l'introduction d'un sujet personnel inverse la tendance. Ce rôle protecteur du pronom sujet personnel animé trouve un certain écho dans une observation de Ploog (2013) qui établit un parallèle entre l'absence de pronominalisation des référents inanimés dans la majorité des langues niger-congo de la région et l'utilisation attestée dans le français abidjanais, des pronoms LE/LUI pour les seuls référents animés :

6 Les autres séquences citées par Moreau n’ont pas été retenues à cause de leur faible présence, elles indiquent néanmoins des tendances similaires : je ne dis pas (4 occurrences en Afrique, 4 pour le reste de la base) vs. je dis pas (I occurrence en Afrique, is dans le reste de la base). 
« Le paradigme clitique objet semble très largement restructuré en ce qui concerne les constituants de troisième personne : les arguments avec un rôle de bénéficiaire et + [animé $]$ sont repris par les clitiques issus du paradigme direct $[\ldots]$, alors que les référents inanimés n'entrent pas dans les positions clitiques, i.e. seront marqués $\varnothing$ » (2013:19).

Dans les locutions (il) fautpas, (il) y a pas, c’est pas le pronom sujet, élément postiche, est sémantiquement vide ce qui l'autorise à former une unité prosodique avec le verbe. Rappelons que les variétés africaines de français ont en partage une prosodie lexicale et non pas post-lexicale comme le français européen. Sous l'influence des langues locales dotées soit d'un accent lexical contrastif ou d'un système tonal (Bordal et Lyche 2012), les français d'Afrique maintiennent un accent lexical ou même un système tonal comme le français centrafricain où les mots lexicaux sont le plus souvent caractérisés par un ton haut sur la dernière syllabe (Bordal 20I2). Il s'ensuit qu'une suite comme je ne pense pas sera susceptible de porter 3 accents, un sur chaque mot, alors que dans ilfaut pas, l'élément postiche il ne constituera pas à lui seul une unité accentuelle. De ce fait dans il ne faut pas, la particule négative ne se trouvera toujours à l'intérieur d'une unité prosodique et qu'elle ne sera pas protégée par sa position. En effet, selon l'hypothèse de marquage (Lacheret, Lyche et Tchobanov 20I2), la position initiale de syntagme constitue une position forte susceptible par exemple d'empêcher la chute d'un schwa. Il suffit d'opposer en effet il est nél(e) 20 juin à il est né le zo juin (et non pas le 22) où la présence du schwa indique le début d'un nouveau syntagme prosodique et entraîne la réalisation de la voyelle. Dans je ne pense pas, un accent sur le pronom personnel placera la particule négative en début d'un autre domaine accentuel, ce qui la protégera contre l'élision ${ }^{7}$. Cet effet est particulièrement sensible avec le pronom nous, qui en français centrafricain, par exemple, est doté d'un ton haut comme toutes les unités lexicales et qui est catégoriquement suivi de

Il n'en reste pas moins vrai que dans une suite de 2 clitiques, le deuxième est affaibli, ce qui se manifeste par un nombre remarquable de chute de schwas dans ce contexte particulier alors que la voyelle $<$ e $>$ est généralement stable dans les français africains (Lyche et Skattum 2012, Bordal 2012). 
la particule ne (Bordal 20I2). Une fois établie la stabilité remarquable de la particule dans les données orales, tournons-nous vers les sms, souvent considérés comme des productions graphiques de l'oral (Ledegen $2007 \mathrm{~b}$ ).

Nous disposons pour Dakar (Sénégal) de quelques données qui concordent entièrement avec ce que nous livre la parole spontanée. Nous relevons dans le corpus de sms de Lexander (20II) les exemples suivants :

(I) Je ne pe pa 2 mand à mé parent

(2) Tu ne pense meme plus a m'appeler

(3) Salut gosse, tu ne viens pa récupéré ta formulaire

(4) Mitemp naniou instant $\mathrm{bi}^{8}$ mais boy il ne joue tiotoudayam ${ }^{9}$ rien $\mathrm{d}$ bon

(s) Dommage k'on n s voi pa

La particule se maintient quasi catégoriquement même dans un registre très familier. Le seul cas où elle est omise concerne la locution faut pas :

(6) boy fautpas oublié les cd des clip.

Nous pouvons donc conclure pour les variétés africaines de français à une présence remarquable d'une négation discontinue là où les autres variétés de français se contentent de l'adverbe $\operatorname{pas}^{10}$. L'absence de la particule reste néanmoins une option dans la grammaire des locuteurs lorsque l'on a affaire à des impersonnels. Il semble que la particule se voit protégée par des facteurs linguistiques et sociolinguistiques : d'un côté, la prosodie lexicale des variétés concernées et le poids du pronom sujet animé protègent la particule en la plaçant en tête d'unité prosodique ; d'un autre côté, le mode souvent purement scolaire d'apprentissage du français met en valeur l'écrit, canal qui maintient la particule. La comparaison des résultats obtenus à Abidjan et Bamako (Tableau 5) souligne une différence attribuée à la vernacularisation du français. Le taux de maintien reste élevé à Abidjan où le français

8 «pour l'instant, nous sommes à la mi-temps ».

9 《 putain de merde ».

Io Rappelons que le territoire nord-américain voit l'aboutissement du changement vers un seul adverbe négatif: Sankoff et Vincent (1977) offrent l'image d'une variété canadienne d'où la particule ne a presque disparu. La Louisiane quant à elle n'a pas de négation disjonctive (Lyche et al. 2010). 
est très présent dans la vie quotidienne locale, mais il l'est beaucoup moins qu'à Bamako où le bambara s'impose dans les interactions quotidiennes. La vernacularisation du français entraîne dans son sillage la perte de la particule.

\section{La Réunion}

La situation sociolinguistique met en contact le français et le créole. Actuellement, le français est presque toujours Li (en partage avec le créole), la langue haute de l'administration et de l'éducation, mais aussi de plus en plus acquis dans son registre familier et ordinaire (Ledegen 2007a: 323-326) ; la situation attestée encore en 1993 a largement évolué :

One of the characteristic features of the local French is its lack of some of the familiar registers found in the metropolitan language. There is little need for them : in situations where the Parisian or Bordelais would change to français familier, the average Martinican or Mauritian can turn to Creole. (Aub-Buscher 1993 : 205)

Notons par ailleurs que dans la variété de français à La Réunion s’attestent des transferts avérés de la phonologie du créole : il en est ainsi par exemple pour la réalisation du r postvocalique ou des groupes consonantiques pour nommer deux traits clairement identifiés (Bordal et Ledegen 2009).

Les données réunionnaises se révèlent, dans le cadre de cette étude du $n e$, globalement proches des données hexagonales, tout en s'en séparant légèrement, comme nous le verrons plus bas. Il ne s'atteste donc que peu d'influence du créole. Nous avons d'ailleurs trouvé un exemple inverse, où un passage créole ${ }^{11}$ est attesté avec le $n e$, alors même que le créole réunionnais en est dépourvu (Cellier 1985):

II L'intonation nous a fait pencher vers l'interprétation du passage en créole; nous aurions pu aussi y voir un passage « flottant » (Ledegen 2013), possiblement reçu comme français ou comme créole, transcrit comme suit, pour montrer la double interprétation possible:

\{avèk [nom] ne dor pa la nwi lé imposib

\{avec [nom] ne dors pas la nuit 
avèk [nom] ne dor pa la nwi lé imposib

(« avec [nom], ne dors pas la nuit, c'est impossible »)

\section{I. Étude du corpus réunionnais PFC}

Les taux d'utilisation de ne se révèlent totalement comparables entre les corpus parisien et réunionnais, oscillant autour de ro\%, à l'exception de l'entretien guidé dans les données parisiennes, où la surveillance semble un peu plus grande :

Tableau I2 : Taux d'utilisation de ne dans les données PFC pour Paris et La Réunion

\begin{tabular}{|l|l|l|}
\hline & \multicolumn{1}{|c|}{ Guidé } & \multicolumn{1}{c|}{ Libre } \\
\hline Paris & $\begin{array}{l}\mathrm{I} 3 \% \\
(35 / 270)\end{array}$ & $\begin{array}{l}9,6 \% \\
(27 / 28 \mathrm{I})\end{array}$ \\
\hline Réunion & $\begin{array}{l}\mathrm{I0}, \mathrm{I} \% \\
(3 \mathrm{I} / 307)\end{array}$ & $\begin{array}{l}\mathrm{I0} \% \\
(9 / 90)\end{array}$ \\
\hline Valeurp & 0,280591506 & 0,913053544 \\
\hline
\end{tabular}

Les séquences préformées s’alignent de la même façon sur les tendances identifiées pour le reste de la base PFC pour les structures impersonnelles il faut, c'est et il y a-comme le montrent les trois tableaux suivants :

Tableau I3 : (il) (ne) faut pas dans les données PFC pour La Réunion et le reste de la base

\begin{tabular}{|l|l|c|l|l|}
\hline & La Réunion & \multicolumn{1}{|c|}{$\%$} & \multicolumn{1}{|c|}{ Reste base } & \multicolumn{1}{c|}{$\%$} \\
\hline fautpas & $10 / 28$ & $35,7 \%$ & $68 / 175$ & $38,8 \%$ \\
\hline il fautpas & $15 / 28$ & $53,6 \%$ & $99 / 175$ & $56,5 \%$ \\
\hline il ne faut pas & $3 / 28$ & $10,7 \%$ & $8 / 175$ & $4,5 \%$ \\
\hline & & & Valeurp & $0,4103182 I$ \\
\hline
\end{tabular}


Tableau I4 : (c') (n') est pas dans les données PFC pour La Réunion et le reste de la base

\begin{tabular}{|l|l|l|l|l|}
\hline & Réunion & \multicolumn{1}{|c|}{$\%$} & \multicolumn{1}{|c|}{ Reste base } & \multicolumn{1}{|c|}{$\%$} \\
\hline c'estpas & I 43/I 44 & $99,3 \%$ & I 805/ I 842 & $98 \%$ \\
\hline cen'est pas & I/ I 44 & $0,7 \%$ & $37 /$ I 842 & $2 \%$ \\
\hline & & & Valeurp & 0,26756995 \\
\hline
\end{tabular}

Tableau I5: (il) (n’) y a pas dans les données PFC pour La Réunion et le reste de la base

\begin{tabular}{|l|l|l|l|l|}
\hline & Réunion & \multicolumn{1}{|c|}{$\%$} & \multicolumn{1}{|c|}{ Reste base } & \multicolumn{1}{|c|}{$\%$} \\
\hline y a pas & $4 / 37$ & I0,8\% & $45 / 507$ & $8,8 \%$ \\
\hline ilyapas & $33 / 37$ & $89,2 \%$ & 43 I/507 & $85 \%$ \\
\hline iln'y apas & $0 / 37$ & $0 \%$ & 3 I/507 & $6,1 \%$ \\
\hline & & & Valeurp & 0,28992053 \\
\hline
\end{tabular}

Mais il en va différemment pour les sujets personnels : à l'instar des données africaines, mais de façon beaucoup moins massive, les ne sont davantage préservés dans le cas de pronoms sujets personnels comme dans les séquences je ne sais pas ( $25 \%$ de maintien) et je ne pense pas (où les chiffres sont très faibles), et ce de façon significative dans les deux cas, même si la faible fréquence du second cas affaiblit le calcul :

Tableau I6 : (je) (ne) sais pas dans les données PFC pour La Réunion et le reste de la base

\begin{tabular}{|l|l|c|l|l|}
\hline & Réunion & $\%$ & \multicolumn{1}{|c|}{ Reste base } & \multicolumn{1}{c|}{$\%$} \\
\hline je saispas & $2 \mathrm{I} / 28$ & $75 \%$ & $\mathrm{I} 520 / \mathrm{I} 644$ & $92,4 \%$ \\
\hline jene saispas & $7 / 28$ & $25 \%$ & $\mathrm{I} 24 / \mathrm{I} 644$ & $7,5 \%$ \\
\hline & & & Valeurp & 0,00065269 \\
\hline
\end{tabular}


Tableau 17 : (je) (ne) pense pas dans les données PFC pour La Réunion et le reste de la base

\begin{tabular}{|l|l|c|l|l|}
\hline & Réunion & \multicolumn{1}{|c|}{$\%$} & \multicolumn{1}{|c|}{ Reste base } & \multicolumn{1}{c|}{$\%$} \\
\hline jepensepas & $2 / 3$ & $66,7 \%$ & $98 / 104$ & $94,2 \%$ \\
\hline jenepensepas & $\mathrm{I} / 3$ & $33,3 \%$ & $6 / 104$ & $5,8 \%$ \\
\hline & & & Valeurp & 0,05696897 \\
\hline
\end{tabular}

Ainsi, le corpus réunionnais s’aligne sur le français ordinaire en général, tout en manifestant quelque sensibilité pour le statut personnel du sujet dans le maniement du ne.

\section{Corpus réunionnais de sms}

Notre corpus contient $15.000 \mathrm{sms}^{12}$ récoltés d'avril à juin 2008 à la Réunion lors de l'enquête Faites don de vos sms à la science; elle présente le premier terrain de l'étude internationale sms 4 science ${ }^{13}$ mise en place par C. Fairon et l'équipe CENTAL de l'université de Louvain-la-Neuve, suite à l'enquête menée en Belgique francophone en 2004 (Fairon, Klein et Paumier 2006). L'analyse est ici effectuée sur 6964 sms, représentant le mois de mai, soit environ la moitié de la récolte. Les données seront comparées avec

I2 Une fois collecté, le corpus est traité afin qu'il réponde aux exigences juridiques et scientifiques : nettoyage du corpus (élimination de messages adressés à l'équipe, de chaînes, d'histoires drôles ...) (faisant passer le corpus brut de près de 25.000 sms à I5.000 sms à analyser), anonymisation de toutes les données personnelles et transcription du corpus en français standard et en Lékritir 77 pour le créole, pour permettre l'exploration logicielle. Enfin, des annotations sur divers phénomènes phonétiques, lexicaux, syntaxiques et graphiques, ainsi qu'un balisage des segments suivant qu'ils sont créoles, français ou « flottants », i.e. potentiellement interprétables comme créoles et comme français (Ledegen 2013), complètent la préparation du corpus.

i3 Cf. <http://www.sms4science.org>. 
l'entretien libre des données PFC étant donné le caractère peu surveillé de ces production $s^{14}$.

Le tableau suivant révèle des pourcentages d'attestation du ne semblables pour les trois corpus : les entretiens libres PFC de Paris et de la Réunion et le corpus de sms contiennent environ Io\% de ne réalisés.

Tableau I8 : Taux d'utilisation de ne dans les données PFC pour Paris et La Réunion, et le corpus Réunion SMS

\begin{tabular}{|l|c|}
\hline & Présence de ne \\
\hline Paris (entretien libre) & $\begin{array}{c}9,6 \% \\
(27 / 28 \mathrm{I})\end{array}$ \\
\hline Réunion PFC (entretien libre) & $\begin{array}{c}\mathrm{I} 0 \% \\
(9 / 90)^{15}\end{array}$ \\
\hline Réunion SMS & $\begin{array}{c}9,9 \% \\
(\mathrm{I} 89 / \mathrm{I} 897)\end{array}$ \\
\hline Valeurp & 0,85277536 \\
\hline
\end{tabular}

L'examen des séquences préformées révèle ici deux séries de résultats contrastées : d'une part, dans les deux séquences, il faut et il ya, le pronom personnel $i l$ manquant constitue l'emploi le plus fréquent dans le corpus sms; cet usage s'oppose au reste de la base PFC (ainsi que les données PFC pour la Réunion), où la structure avec maintien du il mais absence du ne prévaut, et ce de façon significative :

I4 Les locuteurs, majoritairement jeunes (I3 à 27 ans), nous ont envoyé des messages envoyés lors de véritables échanges communicationnels sur un numéro gratuit, le recopiage automatique permettant ainsi d'éviter les biais obtenus lors du recopiage manuel pratiqué habituellement pour ce type d'enquête (corrections, conscientes comme inconscientes).

I5 Dans ce tableau, ainsi que dans les suivants, les données PFC pour la Réunion ne sont indiquées que pour rappel, les calculs confrontant le corpus de SMS avec les données parisiennes, puis avec le reste de la base PFC. 
Tableau I9 : (il) (ne) faut pas dans le corpus Réunion SMS et la base PFC

\begin{tabular}{|l|l|l|l|l|l|l|}
\hline & $\begin{array}{c}\text { Réunion } \\
\text { SMS }\end{array}$ & \multicolumn{1}{|c|}{$\%$} & $\begin{array}{c}\text { Réunion } \\
\text { PFC }\end{array}$ & $\%$ & Base PFC & $\%$ \\
\hline fautpas & $7 / \mathrm{I} 2$ & $58,3 \%$ & $10 / 28$ & $35,7 \%$ & $68 / 175$ & 38,8 \\
\hline il fautpas & $2 / \mathrm{I} 2$ & $16,7 \%$ & $15 / 28$ & $53,6 \%$ & $99 / 175$ & 56,5 \\
\hline ilnefautpas & $3 / \mathrm{I} 2$ & $25 \%$ & $3 / 38$ & $7,9 \%$ & $8 / 175$ & 4,5 \\
\hline & & & & & Valeurp & 0,00209107 \\
\hline
\end{tabular}

Tableau 20 : (il) (n') y a pas dans le corpus Réunion SMS et la base PFC

\begin{tabular}{|l|l|l|l|l|l|l|}
\hline & $\begin{array}{c}\text { Réunion } \\
\text { SMS }\end{array}$ & \multicolumn{1}{|c|}{$\%$} & $\begin{array}{c}\text { Réunion } \\
P F C\end{array}$ & $\%$ & Base PFC & $\%$ \\
\hline y apas $^{16}$ & $\mathrm{I} 6 / 2 \mathrm{I}$ & $76,2 \%$ & $0 / 4 I$ & $0 \%$ & $45 / 507$ & $8,8 \%$ \\
\hline ilyapas & $2 / 2 \mathrm{I}$ & $9,5 \%$ & $4 I / 4 I$ & $100 \%$ & $43 \mathrm{I} / 507$ & $85 \%$ \\
\hline iln'y a pas & $3 / 2 \mathrm{I}$ & $\mathrm{I} 4,3 \%$ & $0 / 4 I$ & $0 \%$ & $3 \mathrm{I} / 507$ & $6, \mathrm{I} \%$ \\
\hline & & & & & Valeurp & $2,1354 E-2 I$ \\
\hline
\end{tabular}

De l'autre côté, nous trouvons des séquences préformées qui s'alignent sur les usages oraux attestées dans le reste de la base PFC et dans les données réunionnaises de la base : les usages sont catégoriques pour c'est pas et je pense pas, et plus variables pour je (ne) sais pas, le maintien du ne se combinant très fréquemment avec des compléments prépositionnels ou phrastiques : je ne sais pas pourquoi je ris / pource week-end / si elle maime,...

I6 L'examen des autres forclusifs avec il y a montre la même prépondérance de la structure sans il impersonnel ni ne:

Tableau 20': il y a avec d'autres forclusifs que pas

\begin{tabular}{|l|l|l|l|l|l|}
\hline & personne & \multicolumn{1}{|c|}{ plus } & \multicolumn{1}{c|}{ que } & rien & \multicolumn{1}{c|}{$\%$} \\
\hline y $a X$ & 7 & 3 & 4 & 5 & $86,4 \%(\mathrm{I} 9 / 22)$ \\
\hline il y $a X$ & 0 & $\mathrm{I}$ & $\mathrm{I}$ & 0 & $9, \mathrm{I} \%(2 / 22)$ \\
\hline il n'y $a X$ & $\mathrm{I}$ & 0 & 0 & 0 & $4,5 \%(\mathrm{I} / 22)$ \\
\hline
\end{tabular}


Tableau 2I : (c') (n') est pas dans le corpus Réunion SMS et la base PFC

\begin{tabular}{|l|l|l|l|l|l|l|}
\hline & $\begin{array}{c}\text { Réunion } \\
\text { SMS }\end{array}$ & \multicolumn{1}{|c|}{$\%$} & $\begin{array}{c}\text { Réunion } \\
\text { PFC }\end{array}$ & $\%$ & Base PFC & $\%$ \\
\hline c'estpas & $\mathrm{I} 55 / \mathrm{I} 55$ & $\mathrm{I} 00 \%$ & $143 / 144$ & $99,7 \%$ & $\mathrm{I} 805 / \mathrm{I} 842$ & $98 \%$ \\
\hline cen'estpas & $0 / 155$ & $0 \%$ & $1 / 144$ & $0,3 \%$ & $37 / 1842$ & $2 \%$ \\
\hline & & & & & Valeurp & 0,07489974 \\
\hline
\end{tabular}

Tableau 22: (je) (ne) pense pas dans le corpus Réunion SMS et la base PFC

\begin{tabular}{|l|l|l|l|l|l|l|}
\hline & $\begin{array}{c}\text { Réunion } \\
\text { SMS }\end{array}$ & $\%$ & $\begin{array}{c}\text { Réunion } \\
\text { PFC }\end{array}$ & $\%$ & Base PFC & \multicolumn{1}{|c|}{$\%$} \\
\hline jepensepas & $\mathrm{I} 5 / \mathrm{I} 5$ & $\mathrm{1} 00 \%$ & $2 / 3$ & $66,7 \%$ & $98 / 104$ & $94,2 \%$ \\
\hline jenepense pas & $0 / 15$ & $0 \%$ & $1 / 3$ & $33,3 \%$ & $6 / 104$ & $5,8 \%$ \\
\hline & & & & & Valeur $p$ & 0,33976065 \\
\hline
\end{tabular}

Tableau 23 : (je) (ne) sais pas dans le corpus Réunion SMS et la base PFC

\begin{tabular}{|l|l|l|l|l|l|l|}
\hline & $\begin{array}{c}\text { Réunion } \\
\text { SMS }\end{array}$ & \multicolumn{1}{|c|}{$\%$} & $\begin{array}{c}\text { Réunion } \\
\text { PFC }\end{array}$ & $\%$ & Base PFC & $\%$ \\
\hline je sais pas & $\mathrm{I} 44 / \mathrm{I} 62$ & $88,9 \%$ & $21 / 28$ & $75 \%$ & $\mathrm{I} 520 / \mathrm{I} 644$ & $92,4 \%$ \\
\hline je ne sais pas & $\mathrm{I} 8 / \mathrm{I} 62$ & $\mathrm{I} \mathrm{I}, \mathrm{I} \%$ & $7 / 28$ & $25 \%$ & $\mathrm{I} 24 / \mathrm{I} 644$ & $7,5 \%$ \\
\hline & & & & & Valeurp & 0,10738962 \\
\hline
\end{tabular}

L'écrit-sms révèle ainsi ses caractéristiques proches de l'oralité, voire une pratique du « relâchement » (Laks 2000) encore plus poussée en figeant faut pas et y a pas, dans la logique de l'économie et de la condensation (écrire rapidement en omettant l'omettable, à l'instar des télégrammes).

Il est un dernier élément qui donne à voir le caractère hybride - oralité + scripturalité - de l'écrit-sms : le sujet nominal, sans reprise pronominale, déclenche habituellement la présence du ne dans le français oral, à cause de son caractère soigné, son appartenance à la « langue du dimanche » (Blanche-Benveniste 2002 : 2014). Dans le corpus sms pourtant, il est très largement attesté sans ne: 
Tableau 24 : Taux de (non-)réalisation de ne après un sujet nominal

\begin{tabular}{|c|c|c|}
\hline & présence & absence \\
\hline sujet nominal & $\mathrm{I} 4,6 \%(7 / 48)$ & $85,4 \%(4 \mathrm{I} / 48)$ \\
\hline
\end{tabular}

Quelques exemples illustrent cette structure très fréquente :

(I) ton cadeau qui ma foi mia rien coûté

(2) quand la fille t'aime pas

(3) la plupart des filles se gênent pas

(4) mon petit frère arrête pas

(5) mon GSM arrête pas de vibrer

(6) ma mère voulait pas

(7) Mademoiselle écoute pas

S'inscrivant dans la logique de l'économie propre à l'écrit-sms, le corpus contient en effet très peu de dislocations à gauche, organisation de l'information qui relève de l'oral (Lambrecht 1994; Blanche-Benveniste 1990 : 55):

(8) ma mère veut pas (sms) versus ma mère elle veut pas (oral, exemple fabriqué)

(9) et ma mère elle me parlait et français et créole quoi (PFC, 974nb)

(Iо) le français, c'est pas de nous ça (PFC, 974ma)

Les structures à sujet nominal ne sont nullement guidées par des raisons pragmatiques particulières comme l'emphase ou l'emploi ludique ${ }^{17}$. Notre corpus de sms combine ainsi l'omission du ne - trait d'informalité - avec le sujet nominal - trait de formalité.

I7 R. van Compernolle (2007) explique ainsi la présence remarquable ( $14,83 \%$ de ne), dans son corpus de tchats, de ne comme particule pragmatique, « the absence of ne [appearing] to be the norm » (2007:262). 


\section{Données réunionnaises : conclusion}

Les séquences préformées se révèlent ainsi très fréquentes dans les données réunionnaises orales, à l'instar des données parisiennes, et le français ordinaire plus généralement. Rappelons toujours la légère sensibilité pour le maintien du ne dans le cas des sujets personnels.

Le corpus des sms a révélé des particularités : d'une part, pour certaines séquences préformées, il pousse, et ce de façon significative, le « relâchement », ou plutôt la condensation, plus loin que les données orales : il en est ainsi pour faut pas et y a pas. De l'autre, il combine des traits d'oralité (absence de ne) et de scripturalité (sujet nominal), dans une logique d'économie, certes, mais aussi d'hybridation : l'écrit-sms se réalise nettement à l'image de l'oral mais bel et bien dans la modalité de l'écrit, où l'organisation de l'information nécessite peut-être moins le « double marquage » de la dislocation à gauche (Blasco-Dulbecco 1999). Ces deux phénomènes révèlent ainsi la tendance « allégeante » de ces nouveaux écrits : absence de ne, combiné avec absence de pronom impersonnel (fautpas, y a pas) et personnel (sujet nominal). Rappelons toutefois que cet allègement n'est pas appliqué pour le corpus sms africain, montrant la nécessité du traitement pluriel.

\section{Conclusion}

Notre étude a montré que dans une situation de français en contact, la Li du locuteur n'exerce aucune influence sur la présence ou l'absence de la particule négative. Le mode d'appropriation du français en Afrique, essentiellement écrit, impose la présence massive du ne. Ce n'est qu'au moment où le français se vernacularise, comme en Côte d'Ivoire, que commencent à germer les tendances lourdes observées dans le français de référence. À la Réunion, une pratique quotidienne du français associée à un contact plus fréquent avec les variétés hexagonales garantit la pérennité du système de référence. 
Parmi les facteurs linguistiques qui interviennent dans la réalisation ou non de la particule, nous avons souligné l'importance de la prosodie en Afrique où une prosodie lexicale supplante régulièrement la prosodie typiquement postlexicale des autres variétés de français. La prosodie protège la particule en Afrique et il se pourrait qu'elle intervienne également dans le maintien observé à la Réunion dans les suites préformées de type je $n e V$. Le français réunionnais se caractérise en effet, tout comme les variétés africaines, par un maintien élevé de schwas dans les monosyllabes (Bordal et Ledegen 2009), facteur qui pourrait venir renforcer le précédent ${ }^{18}$.

Les corpus de sms confortent dans leur ensemble les tendances régionales dégagées. Le souci d'économie aussi bien linguistique que gestuelle n'entraîne pas une raréfaction de la particule dans les sms récoltés au Sénégal, tout à fait conformes aux données orales. A la Réunion, la logique d'économie influe sur l'omission quasi généralisée du sujet vide dans les séquences préformées et sur l'absence de particule après des sujets nominaux, ce que nous avons attribué à la suppression du pronom personnel dans les dislocations.

Ainsi, la confrontation des conditions et des situations de contact différentes du français, sur les terrains de l'Afrique et de La Réunion, selon les modalités de l'oral et de l'écrit d'un registre majoritairement informel, vient souligner une fois de plus l'indispensable appui de l'analyse syntaxique sur la phonologie et la prosodie. S'annoncent ainsi de multiples chantiers systématiquement comparatifs sur la base PFC et les bases de sms qui se multiplient.

\section{Références}

Armstrong Nigel, 2002, « Variable deletion of French ne. A cross-stylistic perspective », in Language Sciences, 24, 153-173.

Armstrong Nigel \& Smith Alan, 2002, « The influence of linguistic and social factors on the recent decline of French ne », in Journal of French language studies, I2, 23-4I. cette piste. 
Ashby William, 1976, « The loss of the negative morpheme ne in Parisian French », in Lingua, 39, 119-137.

Ashby William, 1981, « The loss of particle ne in French : a syntactic change in progress », in Language, 57, 674-687.

Ashby William, 200I, «Un nouveau regard sur la chute de ne en français parlé tourangeau : S'agit-il d'un changement en cours ? », in Journal of French Language Studies, II, I-22.

Aub-Buscher Gertrud, 1993, «French and French-based Creoles : The case of the French Caribbean », in Sanders Carol (Ed.), French today. Language in its social context, Cambridge, Cambridge University Press, 199-2I4.

Blanche-Benveniste Claire, 1990, Le français parlé. Études grammaticales, Paris, Éditions du CNRS.

Blanche-Benveniste Claire, Rouget Christine \& Sabio Frédéric, 2002, Choix de textes de français parlé, Paris, Champion.

Blasco-Dulbecco Mylène, 1999, Les dislocations en français contemporain, Paris, Champion.

Bordal Guri, 2012, Prosodie et contact de langues : le cas du système tonal du français centrafricain, Thèse de doctorat, Université d'Oslo.

Bordal Guri, \& Ledegen Gudrun, 2009, « La prononciation du français à l'Île de la Réunion : évolution des variations et de la norme », in Durand Jacques, Laks Bernard \& Lyche Chantal (Dirs), Phonologie, variation et accents du français, Paris, Hermès Science/Lavoisier, 177-205.

Bordal Guri \& Lyche Chantal, 20I2, « Regard sur la prosodie de français d'Afrique à la lumière de la Li de locuteurs », in Simon Anne Catherine (Ed.), La variation prosodique régionale en français, Bruxelles, De Boeck-Duculot, 179-198.

Boutin Béatrice A. \& Turcsan Gabor, 2009, « La prononciation du français en Afrique : la Côte d'Ivoire », in Durand Jacques, Laks Bernard \& Lyche Chantal (Eds), Phonologie, variation et accents du français, Paris, Hermès Science/ Lavoisier, I3I-I52.

Boutin Béatrice A., Gess Randall \& Guèye Gabriel M., 2012, «French in Senegal after three centuries : A phonological study of Wolof speakers' French », in Gess Randall, Lyche Chantal \& Meisenburg Trudel (Eds), Phonological Variation in French : Illustrations from three continents, Amsterdam, Benjamins, 45-7I.

Cellier Pierre, 1985, Comparaison syntaxique du créole réunionnais et du français : réflexions pré-pédagogiques, St Denis de La Réunion, Université de La Réunion. van Compernolle Rémi A., 2007, «The variable use of ne in public French-language chat », in Gerbault, Jeannine (Ed.), La langue du cyberespace : de la diversité aux normes, Paris, L'Harmattan, 25I-264.

van Compernolle Rémi A. \& Williams Lawrence, 2009, « Variable omission of $n e$ in real-time French chat. A corpus-driven comparison of educational and 
non-educational contexts », in Canadian Modern Language Review, 65/3, 413440 <http://www.personal.psu.edu/ ravi37/preprints/CMLR.negation.pdf> Coveney Aidan, 1996, Variability in Spoken French. A Sociolingistic Study of Interrogation and Negation, Exeter, Elm Bank.

Coveney Aidan, 1998, « Awareness of linguistic constraints on variable ne omission », in Journal of French Language Studies, 8, I59-188.

Cuq Jean-Pierre, 1991, Le français langue seconde : origine d'une notion et implications didactiques, Paris, Hachette.

Diakité Drissa, 2000, « La crise scolaire au Mali », in Nordic Journal of African Studies, 9, 6-28.

Diller Anne-Marie, 1983, « Subject NP structure and variable constraints ; the case of ne deletion », in Fasold Ralph W. (Ed.), Variation in the Form and Use of Language, Washington DC, Georgetown University Press, 167-175.

Durand Jacques, 1993, « Sociolinguistic variation and the linguist », in Sanders Carol (Ed.), French today. Language in its social context, Cambridge, Cambridge University Press, 257-285.

Durand Jacques \& Lyche Chantal, 2013, « PFC et les français périphériques », in Ledegen Gudrun (Ed.), La variation du français dans les aires créolophones et francophones, Tome I, Paris, L'Harmattan, II-29.

Durand Jacques, Laks Bernard \& Lyche Chantal, 2009, « Le projet PFC (phonologie du français contemporain : une source de données primaires structurées », in Durand, Jacques, Laks, Bernard \& Lyche, Chantal (Eds), Phonologie, variation et accents du français, Paris, Hermès Science/Lavoisier, 19-62.

Fairon Cédrick, Klein Jean \& Paumier Sébastien, 2006, Le langage SMS. Étude d'un corpus informatisé à partir de l'enquête « Faites don de vos SMS à la science », Presses universitaires de Louvain, Louvain-la-Neuve, Cahiers du Cental, 3.I.

Fonseca-Greber Bonnie, 2000, The change from pronoun to clitic to prefix and the rise of null subjects in spoken Swiss French, Thèse de doctorat, University of Arizona.

Fonseca-Greber Bonnie, 2007, « The emergence of emphatic ne in conversational Swiss French », in Journal of French Language Studies, 17-3, 249-275.

Hansen Anita, \& Malderez Isabelle, 2004, « Le ne de négation en région parisienne. Une étude en temps réel », in Langage \& Société, 107, 5-30.

Kawaguchi Yuji, 2009, « Particules négatives du français : ne, pas, point et mie. Un aperçu historique », in Baronian Luc \& Martineau France, Le français d'un continent à l'autre, Sainte-Foy, Les Presses de l'Université Laval, 193-210.

Lacheret Anne, Lyche Chantal \& Tchobanov Atanas, 20I2, « Schwa et position initiale revisitée : l'éclairage de la prosodie en phonologie du français contemporain », in Langue française, $169,137-158$.

Laks Bernard, 2000, « De la variation et des variantes : à propos du relâchement 》, in Linx, 42, <http://linx.revues.org/747> 
Lambrecht Knud, 1994, Information Structure and Sentence Form, Cambridge, Cambridge University Press.

Ledegen Gudrun, 2007a, «Inventaire des particularités morpho-syntaxiques du français régional de la Réunion : interférences, 'régionalismes grammaticaux' ou français 'ordinaire' tout court ? », in Le français en Afrique, 22, 319-330.

Ledegen Gudrun, $2007 \mathrm{~b}$, « Résonance SMS. 'Jc c koi mé javé pa rèalizé sur le coup!' », in $\operatorname{Lin} x, 57,<$ http://linx.revues.org/70>

Ledegen Gudrun, 2013, « Prédicats 'flottants' entre le créole acrolectal et le français à La Réunion : exploration d'une zone ambigue », in Chamoreau Claudine \& Goury Laurence (Coord.), Systèmes prédicatifs des langues en contact, Paris, CNRS Editions.

Lexander Kristin V., 2011, Pratiques plurilingues de l'écrit électronique : alternances codiques et choix de langue dans les SMS, les courriels et les conversations de la messagerie instantanée des étudiants de Dakar, Sénégal, Thèse de doctorat, Université d'Oslo.

Lyche Chantal \& Skattum Ingse, 2OI2, « The phonological characteristics of French in Bamako, Mali : A sociolinguistic approach », in Gess Randall, Lyche Chantal \& Meisenburg Trudel (Eds), Phonological Variation in French : Illustrations from three continents, Amsterdam, Benjamins, 73-IOI.

Lyche Chantal, Klingler Tom \& LaFleur Amanda, 2010, « Conversation à la Ville Platte (Louisiane, Etats-Unis) : langue et musique en Louisiane », in Detey Sylvain, Durand Jacques, Laks Bernard \& Lyche Chantal (Eds), Les variétés $d u$ français parlé dans l'espace francophone, Paris, Ophrys, 351-364.

Moreau Marie-Louise, 1986, « Les séquences préformées : entre les combinaisons libres et les idiomatismes. Le cas de la négation avec ou sans ne », in Le français moderne, 54, 137-160.

Ploog Katia, 2013, « Mécanismes discursifs et exigences pragmatiques dans les dynamiques langagières », in $C L A I X, \mathrm{n}^{\circ}{ }_{24}$, Kriegel Sybil \& Véronique Daniel (Dirs), « Contacts de langues, langues en contact », $13-34$.

Pohl Jacques, 1968, « Ne dans le français parlé contemporain : les modalités de son abandon », in Actes du Xe congrès international de linguistique et de philologie romanes, Vol. 2, 1343-59.

Pooley Tim, 1996, Chtimi : the Urban Vernaculars of Northern France, Clevedon, Multilingual Matters.

Poplack Shana, \& St-Amand Anne, 2007, « A real-time window on 19th century vernacular French. The Récits du français québécois d'autrefois », in Language in Society, 36-5, 707-734.

Queffelec Ambroise, 2008, « L'évolution du français en Afrique noire, pistes de recherches », in Holter Karin \& Skattum Ingse (Eds), La francophonie aujourd'hui. Réflexions critiques, L'Harmattan, Paris, 63-76. 
Robillard Didier de \& Beniamino Michel (Eds), 1993-1996, Le français dans l'espace francophone, Tomes i et 2, Paris, Champion.

Sanders Carol, 2004, «Introduction », in Coveney Aidan, Hintze Marie-Anne \& Sanders Carol (Eds), Variation et francophonie. En hommage à Gertrud AubBuscher, Paris, L'Harmattan, 7-10.

Sankoff Gillian, \& Vincent Diane, 1977, « L'emploi productif de ne dans le français parlé à Montréal », in Le Français Moderne, 45, 243-256.

Sankoff Gillian \& Vincent Diane, 1980, « The productive use in ne in spoken Montreal French », in Sankoff Gillian (Ed.), The Social Life of Language, Philadelphia, University of Pennsylvania Press, 295-310.

Tuaillon Gaston, 1983, « Régionalismes grammaticaux », in Recherches sur le français parlé, 5, 227-239.

Valdman Albert (Ed.), 1979, Le français hors de France, Paris, Champion. 

Le français : les mots et les structures 

FRANÇOIS GAUDIN

\title{
Les (nouveaux) mystères de la variation lexicale
}

\begin{abstract}
Chaque fois que deux ou plusieurs interlocuteurs entament une communication, il se met en place une forme de négociation linguistique informelle et plus ou moins tacite, propre à assurer l'intercompréhension. Chacun des interlocuteurs tente de s'ajuster au discours de l'autre : choix de la langue de l'échange, choix du niveau de langue, choix du vocabulaire commun, sens des mots utilisés, etc. L'efficacité de la communication ne peut être effective que si les connaissances linguistiques actives et passives des interlocuteurs se recoupent. Il revient aux terminologues et aux lexicographes de veiller à ce que les ressources linguistiques soient disponibles et accessibles et bien décrites.
\end{abstract}

-ROUSSEAU, 2007:4

Ces ressources sont devenues indispensables à toute communauté linguistique et elles facilitent la négociation tout en permettant de mieux connaître l'étendue des formes à notre disposition pour dire le monde. Leur consultation et leur conception moderne renforcent l'idée qu'une grande variation de formes est offerte aux sujets parlants. Mais le rôle de cette variation nous a paru, à la réflexion, énigmatique. C'est pourquoi, sur un sujet ancien, nous proposons au lecteur d'examiner ces nouveaux mystères.

\section{Au commencement était la variation}

L'approche de la notion de variation, que nous envisagerons dans le cadre de l'étude des vocabulaires et des dictionnaires, doit être replacée dans le cadre scientifique de la théorie linguistique inaugurée par Saussure. Les 
approches apparues après lui, notamment celles de la sociolinguistique, seront contrastées et comprises à partir de cette pensée inaugurale, qui fonde la linguistique moderne européenne. Nous éclairerons les problèmes posés par des exemples tirés de la pratique des lexicographes et examinerons les mystères auxquels cette notion conduit.

\section{Le locuteur, ce drôle d'oiseau}

Comme l'écrivait, en 1956, Marcel Cohen,

Tout homme acquiert son langage ou ses langages dans un entourage social déterminé, désigné ici de la manière la plus générale comme groupement ou groupe. Le langage, avec son fonctionnement général fondamentalement un, est indéfiniment varié suivant les divisions sociales; chaque groupe social a son individualité linguistique. Les degrés de différenciation sont extrêmement variables, leurs effets vont du simple sentiment d'un ou plusieurs traits particuliers jusquà la non-compréhension. (1956:78)

Il continuait en insistant sur le fait que les locuteurs ne se caractérisent pas par une seule appartenance, et que, de ce point de vue, chacun dispose d'une palette d'usages lui permettant de s'adapter aux situations de communication dans lesquelles il est pris. Les interactions verbales sont diverses et chacun actualise de façon singulière les virtualités d'un système labile et hétérogène. Les interlocuteurs diffèrent mais aussi les visées, les intentions, les degrés de connivence, les conventions sociales, etc.

L'homme fait partie suivant les moments de sa vie, suivant les moments d'une journée, de différents sous-groupes. D’une part : couple, enfants et parents, contemporains d'âge, groupes de nations. Chaque intégration à un groupe, éventuellement à plus d'un simultanément, provoque l'emploi de différentes possibilités du langage maternel, quelquefois (cérémonies religieuses, rites d'initiation) l'emploi d'un autre langage. (Cohen $1956: 78$ )

Certains travaux pourraient éclairer l'importance de cette intégration dans la variation, notamment ceux menés par Hélène Thieltges. Ses travaux sur les oiseaux confortent l'hypothèse de l'adaptation sociale dans l'évolution de ces langages animaux et l'absence de relation entre l'habitat et les dialectes. En effet, dans les groupes qu'elle étudie, « le chant portant 
les variations dialectales est un chant intra-sexuel de communication entre mâles » (Thieltges 2013 : 120), comme si la fonction identitaire primait sur les besoins fonctionnels. La communication évolue et, si les « aires dialectales sont conservées d'une année à l'autre », les « dialectes locaux changent en une année voire moins. » Cette stabilité géographique à travers l'évolution des signaux indique la prééminence du groupe constitué et de ses relations avec les autres groupes. L'aspect déterminant de ces changements dans les échanges tient chez ces espèces « hautement sociales, vivant constamment en promiscuité » (ibidem), à leur forte socialisation. De telles observations pourraient éclairer l'étude des communications humaines.

Chez les locuteurs, la pluralité des types d'échanges n'est pas la seule en cause. Il existe dans les répertoires intégrés par les locuteurs des variables qui relèvent de la langue, et liées à des habitudes linguistiques différentes.

Les langues changent tous les jours, elles évoluent, mais à ce changement diachronique s'en ajoute un autre, synchronique : on peut sans cesse repérer dans une langue la coexistence de formes différentes pour un même signifié. Ces variables peuvent être géographiques : la même langue peut être prononcée différemment ou avoir un lexique différent en différents points du territoire. Ainsi un objet aussi simple que la serpillière, pièce de chiffon pour nettoyer le sol, peut aussi s'appeler la panosse (en Savoie et en Suisse), la wassingue (dans le Nord), le torchon (dans l'Est), la since (dans le Sud-Ouest). Un atlas linguistique comme celui de Gilliéron et Edmont nous donne des milliers d'exemples de cette variation régionale. Mais ces variables peuvent aussi avoir un sens social, lorsqu'en un même point du territoire une différence linguistique est plus ou moins isomorphe d'une différence sociale. (Calvet $1993: 65$ )

La formule de Calvet, qui parle de « coexistence de formes différentes pour un même signifié $\gg$, laisse songeur du point de vue de la mise en évidence des invariants, donc des unités linguistiques. Panosse, serpillière et les autres noms cités peuvent-ils être réduits à des variantes ? Quoi de commun entre eux, sinon leur renvoi référentiel - qui n'est pas de mince importance, certes ${ }^{1}$. Ce sont des synonymes, sans doute, mais leurs signifiés sont-ils réductibles les uns aux autres ? On peut en douter.

I C'est la position labovienne, mais on sait les dangers d'une attitude aussi idéaliste. La référenciation constitue une action interactive complexe et la valeur de vérité, avancée aussi par Labov, joue un rôle faible dans les échanges quotidiens. 
Les facteurs qui contribuent à ce que nous disposions d'un nombre, d'ailleurs très inégal selon les secteurs de l'expérience, de vocables pour exprimer ce que nous souhaitons dire, et notamment pour désigner des objets ou des idées, sont variés et interrogent la possibilité même d'invariants dont la recherche est au cœur de l'enquête scientifique. Opposer ainsi variation et invariants ne rend pas justice au fonctionnement lexical. Le fonctionnement de cive et ciboule n'est pas analogue à celui de réacteur à turbine et turboréacteur.

\section{Variation et synonymie}

Concernant la variation, l'un des problèmes que rencontre toute approche du lexique dans une perspective saussurienne est celle du fonctionnement synonymique. Car une question reste pendante : qu'est-ce qui constitue la distinction entre deux synonymes ? On pourra regrouper cette différence en parlant, avec Irène Tamba-Mecz de «modes d'évaluation ou de présentation distincts ». Certains parlent de connotations différentes, mais le terme de connotation, un peu fourre-tout, ne permet pas d'expliquer grand-chose. Les différences entre les synonymes tiennent tout d'abord à trois grands types de variations dûment étudiées : la variation diachronique, liée aux différents états historiques du vocabulaire français, la variation diatopique, liée aux différentes régions de la francophonie, la variation diastratique, liée aux usages des différentes couches sociales. Ex. :

TRAVERSIER : (Canada) Bac, ferry-boat.

T.S.F.: vieilli. Radiodiffusion.

PAF : Pop. Ivre.

BOUFFER : Pop. Manger.

On peut également distinguer selon les styles, les types de discours, les genres, les classes d'âges, les orientations sexuelles, que sais-je ? Cohen dit l'essentiel. Mais, quoi qu'il en soit, ces variations n'épuisent pas l'ensemble des faits de synonymie, loin s'en faut. Il existe également des synonymes dont la coexistence tient à des différences socio-cognitives, entendons par là que les dénominations concurrentes circulent dans des groupes de locuteurs qui appartiennent avec des univers professionnels, ou plus largement à des univers de connaissances différents. 
Par exemple, il existe des oppositions entre « noms vulgaires » et « noms scientifiques ». Il peut s’agir de catégorisations différentes : sont synonymes tigre et felix tigris, chlorure de sodium et sel ou microbe et microorganisme. L'adéquation des signes concernés tient à des univers de discours et des genres de textes. L'énoncé J'ai vu un felix tigris au zoo est déviant. De même, aucune recette de cuisine n'utilisera le nom chlorure de sodium. Il ne faudrait pas réduire ces différences à des seules différences en matière de savoir. Il n'y a pas les mots des ignorants et les mots des savants. Ce sont bien des univers de référence et de discours qui se rencontrent alors qu'on y parle différemment. L'administration est un bon exemple d'univers particulier qui, bien que concernant tout un chacun, a développé un lexique spécifique. Il en résulte, là encore, des relations de synonymie dont les lexicographes rendent compte :

PRÉPOSÉ : Admin. Facteur, factrice des postes.

OMNIPRATICIEN : Admin. Médecin généraliste.

La synonymie tient donc au fait que les descriptions du lexique d'une langue rassemblent des formes linguistiques qui appartiennent à des univers de discours différents, parfois propres à des groupes particuliers. Le lexicographe est là pour décrire des relations d'équivalence ou d'échangeabilité qui existent entre des signes : les synonymies. Le pluriel permet ici de se souvenir que la relation d'équivalence inégale tient au fait que chaque mot est marqué d'une spécificité :
I. Je ne vais pas voir mon omnipraticien, mais mon généraliste.
2. Tu n'attends pas le préposé, mais le facteur.
3. Il n'est pas paf, mais ivre.
4. Nous ne prenons pas le bac, mais le traversier.
5. Vous n'avez ont pas essuyé une drache, mais une averse.
6. Ils n'écoutent pas la T.S.F., mais la radio.

Pour chacun de ces énoncés, l'opposition entre les deux synonymes produit un effet de sens. Il en résulte l'affirmation de la spécificité de chaque désignation : préférence accordée au langage ordinaire sur la langue de l'administration pour les deux premiers exemples, préférence accordée au choix du standard par rapport à la forme populaire pour le troisième, affirmation 
d'une spécificité géographique pour les quatrième et cinquième exemples et, enfin, affirmation d'une spécificité diachronique pour le dernier. Mais un problème théorique demeure : cette relation établie avec soin se situe entre des signes distincts, donc entre des invariants. Quid de la relation entre variation et invariants ? C'est là un des mystères que l'on rencontre en réfléchissant à la question de la variation lexicale.

\section{La variation entre la linguistique et la sociolinguistique}

La notion de variation est très usuelle en sociolinguistique. Elle permet et a permis de mettre en évidence le caractère hétérogène des modes d'existence des langues et de lutter contre l'idée préconçue de la prééminence d'une norme : il y aurait une façon de « bien » parler le français, le corse, le wolof ou le kabyle et les autres façons seraient plus ou moins défectueuses. Pour lutter contre ces préjugés répandus, et réintroduire la légitimité de toutes les façons de parler, la variation est utile. Elle ouvre nos représentations, nos doxas, à la tolérance - par exemple dans l'enseignement des langues.

\section{Déconstruire la langue et la variation}

Toutefois, il convient, dans une démarche critique, de s'interroger sur les limites de ce concept, ou, plus modestement, de cette notion. En effet, son usage fait courir des risques car elle emporte avec elle l'idée qu'il existe une centralité et que la variation tourne autour de ce centre. Et, de ce fait, on tend à considérer ce centre, cette norme comme pré-existant. On procède à une réification. En effet, dans leur existence concrète, les langues ne sont pas toujours déjà données, préexistantes à leur mise en usage. Elles naissent ou renaissent continûment. Et elles existent avec, toujours, leurs réalisations temporelles, spatiales, sociales. Le fait que la normalisation - effectuée par le biais, notamment, de l'enseignement - les minimise et les réduise ne doit 
pas conduire à les oublier. De plus, les langues sont des objets aux limites floues, pas toujours aisées à dénombrer ou à distinguer.

Lorsque l'on parle de variation, il y a toujours, sous-jacente, une norme, abstraite, qui sert de référence et donne sens au mot même de variation. La langue normée, celle que l'on enseigne et que l'on essaie d'utiliser au mieux, est une construction qui arrive après les usages variables que les locuteurs font de la langue. Comme le dit Saussure, la parole est première par rapport à la langue. La variation est donc, elle aussi, première et ne doit pas être appréhendée comme marginale par rapport à la norme qui sert à l'évaluer. Mais, à vrai dire, c'est presque du mot variation qu'il faudrait se passer pour penser correctement. Surtout si l'on conserve présente à l'esprit la quête des invariants.

Le problème réside sans doute en partie dans le fait que ce qui constitue l'unité d'une langue ne réside pas au premier chef dans une structure abstraite mais dans un sentiment linguistique de cohésion. Se dire locuteur d'une langue, c'est partager un lien, un moyen d'échange. « La communauté de langage n'a pas seulement une utilité immédiate dans les rapports continus entre gens vivant ensemble : elle s'accompagne d'un sentiment spécial de coappartenance », comme l'écrivait Marcel Cohen (1956 : 108). Et si la langue était la première structure de sociabilité ? Alors, pour l'approcher, la seule dimension linguistique ne saurait suffire. En effet, poursuit Cohen,

on ne peut juger de la valeur du lien par le langage que si on le met en rapport avec d'autres traits communs et différentiels d'une société ou d'une fraction de société. Au total, c'est un fait qu'à tout groupement d'hommes avec vie commune (ou en partie commune) correspond un langage soit nettement distingué, soit différencié par quelques traits seulement. Inversement, chaque langage dont on a constaté l'existence correspond à un groupement, celui-ci pouvant d'ailleurs avoir été fractionné, ou être de contours flous, ses membres s'étant intégrés ou étant en train de s'intégrer à d'autres divisions d'une société. (Cohen 1956: 108)

Or une politique de la norme s'est imposée durablement pour le français qui relève d'une forme d'oligarchie linguistique. On crée un espace de référence sociolinguistique qui, légitimé par Vaugelas et ses successeurs ainsi que par l'Académie française, se met en place en imposant une norme 
nouvelle. Celle-ci est fondée sur des règles qui sont, dans une large mesure, arbitraires et sont justifiées

a) par l'étymologie - que ne connaît pas le locuteur moyen -,

b) par une esthétique - mais qu'est-ce que le beau en matière de langue sinon une caution accordée à un usage particulier ?-,

c) par la filiation avec d'autres langues, notamment le latin - que ne connaît pas plus le locuteur moyen -, et finalement

d) par la légitimité de certains locuteurs auxquels est accordé un statut de prescripteurs, principalement les « bons auteurs ».

Donc au sentiment de co-appartenance, on vient substituer la soumission à une oligarchie linguistique. L'objectif, qui résulte d'un effet de système explicable par l'histoire, est au fond de proposer à tout un chacun de calquer son usage sur un modèle donné en exemple, qui apporte une plus-value dans le monde social. Finalement, c'est la recherche de la «distinction » que l'on peut entendre dans son sens usuel, comme dans celui du concept illustré par le sociologue Pierre Bourdieu. Pour le dire vite, la promulgation et la diffusion institutionnelle de cette norme créent les conditions de l'instauration de rapports de domination linguistique, dans lesquels une partie des formes lexicales relevant de la «variation » va souffrir de minoration. Car les sanctions du «marché linguistique » vont conduire les locuteurs à adhérer à un ensemble de valeurs qui leur sont insensiblement inculquées, au travers d'un long et lent processus d'acquisition, au fil des générations. Peut-être que nos variantes, nos synonymes, ne tiennent quà des différences de places sur le marché du sens...

\section{Attention, norme utile!}

Toutefois, il ne faudrait pas simplifier à outrance et jeter la norme avec l'eau du bain linguistique, si j'ose dire ... En effet, bien que la place du français fût, au XVI ${ }^{\mathrm{e}}$ siècle, encore limitée, il a résulté de cette entreprise menée par le pouvoir parisien cette unification linguistique qui a créé un 
espace d'intercompréhension très homogène, alors qu'au sein des espaces linguistiques correspondant aux états allemands et italiens les variétés co-existaient. Ensuite, la Révolution française considérera la langue française comme le ciment de l'unité nationale. Elle marquera le début d'une volonté d'éradiquer les patois. Ce qui n'est pas bel et bon, certes; mais qui a contribué puissamment au destin international du français et au travail que les générations successives ont effectué sur cette langue. Car les langues sont aussi des objets collectifs extérieurs aux locuteurs qui incorporent un travail historique.

En effet, l'idée selon laquelle la langue française est claire, pure, etc. toute cette idéologie aisée à dénoncer - a possédé une efficacité réelle sur la langue elle-même, car ceux qui y ont adhéré ont façonné en ce sens la langue elle-même. Certains ont vraiment cru au « génie » de la langue française et, du coup, on a ciselé un outil. Par exemple, pour le lexique, la langue française a bénéficié, à partir des travaux de l’abbé Gabriel Girard (1677-1748), d'une riche tradition de synonymistes qui ont contribué à ce que des unités lexicales possiblement concurrentes trouvent chacune une place et servent à produire des « programmes de sens » précis et subtilement distingués. Ce travail métalinguistique peut être vu comme un processus d'affinement des contrastes entre invariants; et, si l'on s'accorde avec l'idée que la richesse de mots permet la richesse de la pensée, on mesure l'importance de l'équipement lexical et des nuances qu'il autorise. Cette idée a été suivie si loin que Lafaye, en 1853 , pouvait écrire du français que « c'est peut-être la seule langue qui ne connaisse point de synonymes » (1853: XXIX). Le propos est idéologique, bien sûr. Mais Lafaye connaissait tout de même bien la question.

C'est donc une norme pourvue d'une histoire bien spécifique que l'école républicaine va diffuser et inculquer au cours du XIX ${ }^{e}$ siècle, éliminant les parlers régionaux tout en accordant une place importante à l'enseignement de la langue française. La diffusion de la norme sera également assurée par de nombreux ouvrages, grammaires, dictionnaires, dans des rubriques linguistiques dans les journaux, dans des traités de correction grammaticale, etc. Et tout cet échafaudage reposera sur une hiérarchie des usages très claire et très trompeuse. En effet, le modèle « suprême » est constitué par les écrivains, mais les écrivains édités, en ce sens où les 
manuscrits d'une bonne part d'entre eux sont corrigés par des professionnels. Et ces correcteurs usent de manuels qui sont eux-mêmes fondés sur ... les usages des écrivains. Notons que le ministre Guizot, synonymiste occasionnel, contribua fortement à rigidifier les exigences orthographiques pour les recrutements de l'État. Ceci pour dire qu'il faut à la fois être conscient de l'importance de la norme, de son efficacité sociale et de son rôle dans la communication, tout en ayant à l'esprit qu'il s'agit d'une construction sociale et historique.

La notion de norme, je l'ai évoqué, est très utilisée en sociolinguistique, discipline qui postule que cette variation n'est pas aléatoire mais s'explique par l'appartenance sociale des locuteurs, dans le cadre d'une communauté linguistique. Et c'est l'un des points qui pose problème, car comment délimiter la nature ou l'extension de cette communauté de locuteurs. Qui parle « la langue », le français, par exemple ? Existe-t-il un français international de la francophonie, de la France ? Que se passe-t-il lorsque des États négocient autour d'une norme commune, comme c'est le cas dans la lusophonie ? La communauté linguistique concernée est-elle nationale ? métropolitaine ? régionale ? L'idiolecte est-il le niveau pertinent, comme le disait Meillet?

Parmi les facteurs sociaux pouvant expliquer la variation linguistique, la sociolinguistique interroge les différences d'âge, de profession, de sexe on parle de langages des jeunes, de langages des femmes, de langages de métiers, etc. Mais procédant ainsi, on court le risque de se polariser sur un seul des facteurs qui caractérisent un individu. Comment faire autrement ? Et qu'explique-t-on ainsi ?

\section{Des formes et des représentations}

Depuis les premiers travaux de William Labov, de nombreuses enquêtes sociolinguistiques menées sur différents terrains ont démontré que la classe sociale, le style, l'âge, le sexe, etc. agissaient comme structurant la variabilité des emplois de certaines variantes linguistiques. Je n'y reviens pas. Mais ne retrouverait-on pas là des oppositions séculaires entre les sexes, les classes, etc. dont les aspects linguistiques ne seraient guère spécifiques ? On s'en 
doutait un peu auparavant, mais sans les prestiges réunis de la science et de la langue anglaise. Pour enrichir cette perspective, on peut considérer, avec les sociolinguistes contemporains, que les langues sont faites de formes et de représentations. Cela signifie que, lorsque nous parlons d'une langue, ce qui existe dans la réalité ce sont, d'une part, des usages linguistiques de locuteurs qui se comprennent, ou qui disent se comprendre, et d'autre part, des opinions sur ce qu'est la langue qu'ils parlent, comment il la faut parler, qui la parle bien, etc. Or que devient la variation lorsque l'on raisonne ainsi ?

Quand je dis que je parle français, c'est évident pour vous. Mais deux francophones, l'un vietnamien, l'autre québécois, qui se croisent dans un aéroport et possèdent une compétence très limitée, auront-ils le sentiment de parler français, ou le même français, alors qu'ils parlent de la grève en recourant occasionnellement à des mots d'anglais et d'espagnol ? Dans des situations plus simples, quand corrige-t-on quelqu'un qui ne parle pas comme vous ? On corrige sans sourciller un enfant qui apprend le français. Mais la même erreur commise par un adulte avancé pourra être acceptée pour ne pas le froisser ? Jusqu'où un enseignant accepte-t-il les variétés extrahexagonales du français ? Dire essencerie, est-ce une erreur à corriger en disant station-service, ou un africanisme qui enrichit le francophone de France ? Autre exemple, la télévision nous montre parfois des locuteurs francophones dont les propos sont sous-titrés alors qu'ils sont parfois aisément compréhensibles, ce qui pose la question de la représentation linguistique que les producteurs se font des téléspectateurs, de leur tolérance à la variation dans la représentation du français.

Ces deux niveaux, de formes et de représentations, interfèrent car la façon dont on utilise sa langue, ou ses langues, n'est pas indifférente aux façons dont on se représente cette langue, ce qu'on pense d'elle et des façons de l'utiliser. Si l'on demande à des étudiants plurilingues d'énumérer les langues qu'ils parlent, certains vont oublier d'indiquer qu'ils pratiquent l'arabe ou le berbère, ou le wolof, parce qu'ils ont intégré la minoration dont leur langue est l'objet. Vous interrogez des scientifiques sur le vocabulaire qu'ils utilisent entre eux quand ils parlent français, et non anglais comme dans les congrès, ils se détournent en disant : « non, ce n'est rien, c'est notre jargon », alors que ce sont peut-être des mots utiles 
qu'il faudrait diffuser auprès des étudiants qui peinent parfois à traduire les termes anglais pour écrire leurs mémoires ou leurs thèses. Un exemple amusant : monsieur Southern pose des problèmes aux francophones. Il a donné son nom à une technique (Southern blot) que l'on voit traduite par transfert selon Southern, ce qui est raisonnable, ou désignée par buvardage (qui ne rend compte que de to blot), mais on peut aussi rencontrer, plus curieusement, les formes transfert sudiste, voire transfert sudiste d'ADN! Une telle confusion n'est qu'un des effets d'une francisation trop résolue et dispersée, qui conduit à rencontrer des concurrences fâcheuses. La technique dite PCR (Polymerase Chain Reaction) peut être siglée en français $R C P$ (réaction en chaîne parpolypérase) - les mêmes lettres inversées, ce qui est fréquent - mais aussi $A C P$ (Amplification en Chaîne par Polymérase), si l'on suit les recommandations officielles. Ces exemples montrent qu'existe une créativité, dispersée, de mots français qui pourraient rendre service s'ils circulaient mieux et sont dévalorisés en raison du prestige dénominatif de l'anglais (cf. Pruvost 1994).

Cette prise en compte des deux niveaux que nous venons de distinguer était déjà présente dans les travaux pionniers de Labov :

Il serait faux de concevoir la communauté linguistique comme un ensemble de locuteurs employant les mêmes formes. On la décrit mieux comme étant un groupe qui partage les mêmes normes quant à la langue. (1976:228).

Mais parler des « normes quant à la langue », est-ce parler des langues ou des représentations ? Et les deux vont-ils de pair ? Son propos reposait sur une enquête et une analyse de traits phonétiques, mais il vaut pour toutes les dimensions du langage, et bien sûr pour le lexique. En effet, comme l'écrit Alain Rey,

le lexique est un bon terrain pour cet exercice. La variation des sons, des « accents », est difficile à décrire et se heurte à d'intimes préjugés; celle des règles de la grammaire est subtile, et son acceptation est limitée par la nécessaire unification pédagogique. Celle des mots, en revanche, mieux acceptée, a l'immense avantage de donner sur les réalités humaines, telle une fenêtre grande ouverte sur la variété des paysages culturels. (Rey $1988: 4$ ) 
Car étudier la variation, ce n'est pas seulement mettre la loupe sur un phénomène social et linguistique, c'est comprendre comment apparaissent et se gèrent les différences et les phénomènes de pouvoir qui leur sont associés. Si l'on s'arrête sur le cas du français, on peut insister sur le fait que l'étude de la variation permet de comprendre et de mettre en valeur la richesse que lui confèrent ses usages provinciaux et extra-hexagonaux et contribuent notablement au patrimoine international qu' il constitue.

De franchir ses cours d'eau originels, puis les provinces, les frontières, enfin les mers, le français s'est enrichi et assoupli. Si, dans le même temps historique, il a pu se dégrader, se compromettre, c'est plutôt de l'intérieur, car les modulations lointaines ont fourni à notre langue les moyens de réagir contre les appauvrissements et les facilités. (Rey $1988: 5$ )

Cette diversité est une chance car nous parlons pris dans des doxas ; l'esprit critique nous aide à les tenir à distance mais la langue elle-même est une doxa dont il est malaisé d'estomper l'empreinte et la diversité de ses réalisations nous aide, différemment à la diversité des langues, à relativiser notre place dans le bruissement incessant du marché des langues.

Les ressources linguistiques que conçoivent les lexicographes et les terminographes pour, notamment, faciliter nos négociations enregistrent une multitude de formes, invariants et/ou variantes, pris dans des phénomènes que nous faisons relever de la variation. Ces recueils nous permettent de découvrir d'autres voies pour nous dire, d'autres chemins de pensée.

Mais les formes qui y sont présentées comme étant en concurrence sont prises aussi dans la loi de répartition mise en évidence par Bréal, selon laquelle les synonymes tendent à être résorbés et soit sont distingués, soit disparaissent.

Pour Bréal, cette autorégulation procède de l'esprit humain ou, plus précisément, de l'intelligence populaire, véritable instinct du langage, force obscure, décisive en matière d'évolution linguistique, et qui se résume en définitive au souci de comprendre et d'être compris (Nyckees $2006: 63$ ).

Mais peut-on pour autant renvoyer la variation à des stades préalables, où la loi de répartition n’aurait pas « encore » joué son rôle? 


\section{La variation diachronique et la variation diatopique}

On pourrait se demander : pourquoi les langues changent-elles ? Si le système linguistique est efficace, si l'on parvient à se comprendre, pourquoi se modifie-t-il ? En fait, ce serait considérer le changement comme une anomalie à expliquer. Or l'usage du langage évolue en permanence, change tout le temps : les circonstances, les interlocuteurs, les situations, les buts, les actions visées, etc. Face à cette diversité pourquoi y aurait-il de l'uniforme, de l'unique ? Et c'est pourquoi la recherche d'invariants est si stimulante.

La diversité que nous constatons connaît plusieurs axes. Les deux principaux sont diachroniques et diatopiques. Le temps et l'espace qui nous déterminent, ici et maintenant.

\section{La variation diachronique}

Lire un texte du XVIII e conduit à rencontrer des formes inconnues des usages contemporains, formes disparues ou sens tombés en désuétude. De même, un texte technique contemporain met le lecteur non spécialiste en position de découvrir des signes nouveaux et des significations inconnues de lui, par exemple lorsqu'on lui parle de « la microstructure de matériaux céramiques relaxeurs et l'analyse par diffraction à haute énergie de transitions de phases. »

L'évolution d'une langue se déroule de façon continue et se trouve même au principe du fonctionnement des langues. La plupart des changements se passent de façon inaperçue, sans rupture. Dans l'évolution des mots, il existe perpétuellement une coexistence entre des emplois plus anciens et des emplois plus actuels ou nouveaux. Les choses changent et peuvent conserver les mêmes noms, elles peuvent demeurer identiques et se trouver désignées autrement. Derrière le théâtre mouvant où se jouent les relations entre les signes et le monde, un niveau persiste. Il doit être constitué, pour fonctionner, d'invariants, relativement pérennes malgré les aléas qui marquent l'histoire de leurs utilisations. 


\section{Phénomène intéressant: le diachronisme}

Examinons cette citation d'Alfred Delvau :

Des engagements sérieux eurent lieu sur plusieurs points de Paris et dans le rayon des Batignolles. Les barricades élevées avec des haquets de porteurs d'eau, des camions, des fiacres, des voitures de transports, avec tous les engins de barricades enfin ... (Delvau, Histoire de la révolution de février, I850: I6I)

Le nom haquet désigne un type de véhicule hippomobile, long et étroit, servant à transporter des tonneaux, qui est devenu désuet. Le nom fiacre désigne un autre type de véhicule hippomobile, fermé à quatre places, quatre roues et suspension plus connu aujourd'hui car il a été mieux conservé par la mémoire littéraire, laquelle s'intéresse peu au transport des tonneaux. Le nom camion est plus intéressant car sa désignation de véhicule servant à transporter des marchandises - à l'origine une charrette dont les roues avaient très peu de hauteur et qui servait au transport des colis, des pierres, des barriques - a été conservée malgré le changement technique qui a vu apparaître des camions qui n'étaient plus « hippomobiles » mais « automobiles ».

Ce type de différences est banal. Il se rencontre à la lecture de textes un peu anciens dans lesquels coexistent des sens différents de ceux utilisés en synchronie. Il se produit également en synchronie, quand des communautés utilisent des états de langue différents. Le cas du français canadien comme conservatoire de formes disparues en français de France est un exemple. Cela a-t-il déjà un nom ? J'appelle cela par commodité des diachronismes. Cela relève-t-il de la variation ? Pour chaque position de locuteur, il s'agit d'un invariant; pour le Canadien qui me glose ce qu'il entend par breuvage, ou pour Delvau, rien n'est variable.

Ces phénomènes de changement peuvent être plus ou moins volontaires et correspondre à l'exercice d'un pouvoir sur le vocabulaire. Les raisons peuvent en être très diverses. Les mutations du monde économique, administratif et politique s'accompagnent de décisions lexicales qui ne sont pas sans influencer les façons de catégoriser le monde social, ni sans bouleverser les enjeux qui passent par la langue ; ce faisant, on change les réalités ou au moins les façons de les présenter, donc de les percevoir. 
Des mots nouveaux se substituent aux anciens: on ne parle plus de postes ou de tâches, mais de missions, de rôles ou de fonctions; plus de qualifications mais de compétences et de capacités ; plus d'ouvriers mais de conducteurs. (Tessier 1997 : 297)

L'extension du modèle de la privatisation a gagné de nombreux domaines de l'économie et du social. Une des conséquences a été un recul des termes d'usager, pour les services publics, et de patient, pour la médecine, au profit du seul terme de client qui tend à unifier toutes attitudes dans le seul moule de l'acte marchand. Et dans Les démarches qualité dans l'enseignement supérieur en Europe, Anne Heldenbergh intitule une section de son livre « L'étudiant : un 'client' particulier » (2007: 130).

L'analyse des mutations lexicales exercées sous l'influence des pouvoirs a suscité ces dernières années un certain nombre d'ouvrages (Dewitte 2007; Hazan 2006 ; Tevanian \& Tissot 2010 ; Rey 20II). Ecoutons ce témoignage de Victor Klemperer, auteur d'un témoignage exceptionnel sur l'influence du nazisme sur la langue allemande :

A l'avenir, le mot migrant ne dégagera pas forcément l'odeur de charogne du Troisième Reich. Il en va tout autrement de camp de concentration. J'ai entendu ce mot quand je n'étais encore qu'un jeune garçon et, à l'époque, il avait une résonance pour moi tout à fait exotique et coloniale, pas du tout allemande (compounds où les Boers étaient surveillés par les Anglais). A présent soudain resurgi, il désigne une institution allemande, un dispositif durable ... Je crois quà l'avenir, où que l'on prononce le mot camp de concentration, on songera à l'Allemagne hitlérienne, et seulement à l'Allemagne hitlérienne. (Klemperer $1996: 65$ )

Cette cruelle prescience démontre que les mots peuvent ne changer ni de signification ni de visée référentielle, mais changer de valeur car l'histoire humaine en détermine les conditions de production de sens et de circulation sociale.

Concernant l'histoire des idées, suivre le parcours d'un mot peut éclairer tout un ensemble de changements culturels. Ainsi, en français, le mot révolution était très courant au XVIII ${ }^{e}$ siècle. Utilisé par l'astronomie, il était déjà employé au sens politique au XVII siècle. De nombreux livres s'intitulaient La révolution de tel ou tel pays, désignant ainsi les changements politiques brusques: Histoire de la dernière révolution des Etats du Grand Mogol, par François Bernier, 1670 ; Histoire de la révolution de 
Siam arrivée en l'année I688, par Vollant des Verquains, 1691; Histoire de la dernière révolution des Indes orientales, par Jean-Baptiste Le Mascrier, 1757, etc. Mais il n'empêche que, un certain jour de 1789 , le mot français révolution, sans changer de sens, sans changer de forme, sans changer de prononciation, est devenu tout autre chose. Il est devenu tout d'un coup un symbole, pour certains un symbole d'espoir et pour d'autres un symbole de crainte et de « terreur », le mot terrorisme étant d'ailleurs une création révolutionnaire. Tout ceci a été magnifiquement étudié par Alain Rey dans Révolution. Histoire d'un mot (1989).

Marie-France Piguet a mené une étude ${ }^{2}$, de moindre ampleur mais analogue, sur le mot classe - qui a connu un tel engouement dans le vocabulaire sociologique et politique. Elle y montre comment on passe d'une nébuleuse de sens instable et floue au syntagme figé de « lutte de(s) classes ». Pour ce faire, elle passe en revue les définitions du mot classe dans les dictionnaires et la diversité des emplois de ce terme dans un ensemble de textes provenant, pour l'essentiel, d'économistes et d'historiens français des XVIII et XIX ${ }^{\mathrm{e}}$ siècles.

Ici, ce ne sont plus les diverses façons de désigner qui sont en cause, mais la variabilité des emplois qui conduit le linguiste à articuler les acquis des nombreuses études sur la polysémie lexicale à l'histoire des idées. Les exemples choisis relèvent des champs intellectuel, social, politique, ce qui les singularise sans doute; mais ils montrent que les inflexions des sens produits avec des formes résultent de jeux de pouvoir. Peut-on penser ces phénomènes en parlant encore de variation ? Et quelle communauté de parole utiliserait quel(s) invariant $(s)$ dans ces usages mêlés ? Le mystère demeure.

\section{La variation diatopique}

A ce changement diachronique s'en ajoute un autre, synchronique : on peut sans cesse repérer dans une langue la coexistence de formes différentes pour un même usage désignatif, ou pour produire une signification analogue.

2 Marie-France Piguet, 1996, Classe. Histoire du mot et genèse du concept, des physiocrates aux historiens de la Restauration, Presses Universitaires de Lyon, 196 p. 
Ces variables peuvent être géographiques, on parle alors de variation diatopique. Elle prend en compte les traits relevant de la différenciation d'une langue selon les régions; on parle alors de régiolectes, de topolectes ou de géolectes. On peut englober l'ensemble de ces formes sous le nom de régionalismes. Ils ont été pris en compte dès le début de la lexicographie française - Furetière (I690) en enregistre. Il est vrai qu'au fil des siècles, ils ont été parfois perçus, mais pas toujours, comme des « fautes » dont il fallait se défaire, partageant l'opprobre dont souffraient les langues régionales, surtout à partir de la fin du XVIII ${ }^{\mathrm{e}}$ siècle. Renvoyons, par exemple, au Dictionnaire du bas-langage ou des manières de parler usitées par le peuple, de Charles-Louis d'Hautel ( $18 \circ 8$ ).

L'étude de ces variations linguistiques, appelées aussi diatopismes, selon leur répartition dans l'espace a donné naissance à une tradition ancienne, celle de la dialectologie. Un de ses buts était de dessiner une géographie des aires linguistiques, en établissant des liens entre la distribution spatiale des formes linguistiques et des groupes linguistiques. Les différences étaient abordées, avant la lettre, dans des processus de type sociolinguistique. Cette ancienneté explique que la variété diatopique soit présente à la conscience des locuteurs et bien décrite. Elle est présente dès les origines de la lexicographie française. Et elle n’a jamais quitté ses préoccupations. D’ailleurs, les recueils de régionalismes constituent depuis longtemps un secteur éditorial florissant.

La dialectologie et les recherches qui l'ont continuée en changeant d'étiquette relèvent-elles de la variation ? On découvre, en parcourant ces atlas qu'ils subsument des communautés de parole circonscrites et variées dont les modalités de désignation diffèrent. Mais la signification de ces unités, leurs valeurs, peuvent-être être réduites à leur désignation ? Les noms traversier et bac sont-ils des variantes?

La difficulté est redoublée par l'expansion internationale du français. En effet, son extension géographique a été continue jusqu'au $\mathrm{XX}^{\mathrm{e}}$ siècle et la description des variétés de la France a été enrichie des variétés hors de l'Hexagone au fur et à mesure de la colonisation et de l'extension de la diffusion du français. Dès lors, la notion de variation diatopique perd jusquà sa consistance tant on sent qu'elle engloberait dans une langue des usages hétérogènes et qu'il s’agirait alors d’un français chimérique ne correspondant à aucune communauté de parole effective. 


\section{La variation sociolectale et professionnelle}

L'homo sapiens est aussi un homo faber. Et l'on parle également de variation pour les vocabulaires professionnels. Objet d'étude de la sociolinguistique depuis ses débuts en France, les technolectes sont considérés aujourd'hui comme un type de discours utilisé dans les situations de communication fonctionnelle, et particulièrement les situations professionnelles. Il s'agit d'un sous-système linguistique concernant un champ d'expériences particulier, caractérisé par des usages linguistiques spécifiques car liés à des domaines disciplinaires et sociaux déterminés.

Mais cette notion doit être replacée dans un cadre linguistique pensé et non pas être réduite au seul vocabulaire. Car tout technolecte correspond à un discours spécialisé, et consiste dans l'usage d'une « langue spécialisée », comme le dit Lerat, mais utilisée dans un ensemble de situations de communication que partagent les membres d'une communautétechnique et scientifique ${ }^{3}$.

Dans le cas du français, la variation tient à l'extension internationale de la langue et au fait qu'en dépit d'un désir de standardisation, les usages technolectaux sont partie prenante de situation de contacts de langues ${ }^{4}$. Cette variation internationale, concernant les domaines scientifiques et techniques, n’a été guère étudiée. Il s'agit d'ailleurs moins, selon nous, de variation que de coexistence de formes n'ayant pas l'occasion d'être mises en concurrence. Un des phénomènes explicatifs réside dans la mise en circulation, dans ces discours, de traductions non concertées de termes étrangers, et surtout anglais. Ce problème, évoqué précédemment, se situe au croisement de la politique linguistique et de la terminologie et appellerait une étude socioterminologique.

Plus largement, la diversité des formes dans les terminologies se laisse moins aisément réduire à de la variation, car la dimension cognitive oblige à prendre en compte la dimension conceptuelle. On met donc assez spontanément l'accent sur l'usage des ressources lexicales et non sur le lexique lui-même. communauté, cf. Gaudin (2003). 


\section{Conclusion}

Pour terminer ce parcours, il n'est guère possible de conclure. La variation lexicale demeure un phénomène rétif à une analyse attentive. Les mystères qu'elle recèle tiennent en partie au fait qu'il s'agit d'une notion fondée sur le sens commun, et pertinente dans cette mesure, mais difficile à cerner. Elle opère mieux sur les signifiants, exclus des illusions de la relation dénominative, ou les grammèmes. Tout un chacun répugne un peu à dire que car et parce que relèvent de la variation, même s'ils servent souvent à la même chose. Pour le vocabulaire, dire que l'usage de signes, donc d'invariants, relève de la variation lorsqu'ils sont utilisés dans des circonstances de discours différentes constitue un abus de langage.

La variation relèverait donc plutôt des usages que du lexique. Les ressources dont nous parlions en ouverture, lexicales et terminologiques, jouent un rôle de trésor, recueillant des invariants et des variantes, et subsument les pratiques les plus hétérogènes. La variation serait mieux dénommée comme sociolexicale, car elle réside, dans son principe, chez les locuteurs eux-mêmes et non dans une hypothétique langue. Connaître et utiliser des formes nouvelles, ce n'est pas tant ajouter au lexique qu'augmenter sa façon de comprendre le monde et d'enrichir les partages quotidiens avec nos semblables. Car si distinguer la vanité, la fierté et l'orgueil repose sur un travail de conceptualisation fin et culturalisé, boire un coup et s'en jeter un, ce n'est pas tout à fait se poser de la même façon comme buveur devant son interlocuteur.

Les mystères de la variation tiennent donc en bonne part à la confusion entre le niveau linguistique et celui de l'énonciation. Une mise en équivalence rapide oublie parfois que les signes sont des invariants entre lesquels des réseaux linguistiques et des connivences sociales permettent de créer une relation d'échangeabilité. Et cette échangeabilité ne peut être réduite à une relation d'équivalence, les valeurs étant distinctes sur les marchés linguistiques où ils circulent.

Quant à assimiler ces concurrences de vocables à des différences de réalisation de phonèmes, il y a sans doute un pas. 


\section{Références}

Bavoux Claudine \& Gaudin François (Eds), 200I, Francophonie et polynomie, MontSaint-Aignan, Publications de l'Université de Rouen.

Calvet Louis-Jean, 1993, La sociolinguistique, Paris, PUF (Coll. « Que sais-je ?», $\mathrm{n}^{\circ}$ 273I).

Cohen Marcel, 1956, Matériaux pour une sociologie du langage, Paris, Albin Michel.

Delvau Alfred, I850, Histoire de la révolution de février, Paris, Blosse et Garnier frères. Depecker Loïc, 1990, Les mots de la francophonie, Paris, Belin.

Depecker Loïc, 1995, Dictionnaire du français des métiers. Adorables jargons, Paris, Le Seuil (Coll. « Point virgule »).

Dewitte Jacques, 2007, Le Pouvoir de la langue et la liberté de l'esprit, essai sur la résistance au langage totalitaire, Paris, Michalon.

Gaudin François, 1994, « L'insécurité linguistique des scientifiques. A propos d'une enquête socioterminologique », in Cahiers de lexicologie, 65, 47-58.

Gaudin François, 2003, Socioterminologie. Une approche sociolinguistique de la terminologie, Louvain-la-Neuve, Duculot (Coll. « Champs linguistiques »).

Gaudin François \& Guespin Louis, 2000, Initiation à la lexicologie française. De la néologie aux dictionnaires, Louvain-la-Neuve, Duculot (Coll. « Manuels »).

Girard Gabriel (abbé), 17 I8, La Justesse de la langue françoise, ou les différentes significations des mots qui passent pour synonymes, Paris, Vve d'Houry.

Hazan Eric, 2006, LQR : la propagande du quotidien, Paris, Raisons d'agir.

Heldenbergh Anne (Dir.), 1997, Les démarches qualité dans l'enseignement supérieur en Europe, Paris, L'Harmattan.

Klemperer Victor, 1996, LTI, la langue du IIIe Reich, Paris, Albin Michel (Coll. « Agora »).

Labov William, 1976, Sociolinguistique, Paris, éd. de Minuit.

Lafaye Bernard, I853, Dictionnaire de synonymes de la langue française, Paris, Hachette.

Ledegen Gudrun \& Léglise Isabelle, 2013, « Variations et changements linguistiques », in Simonin Jacky \& Wharton Sylvie (Eds), Sociolinguistique du contact. Dictionnaire des termes et concepts, Lyon, ENS éditions, 315-329.

Messaoudi Leïla (Coord.), 20I2, Sur les technolectes, Rabat, Publications du laboratoire Langage et société.

Neveu Franck, 2004, Dictionnaire des sciences du langage, Paris, Armand Colin.

Nyckees Vincent, 2006, « Rien n'est sans raison : les bases d'une théorie continuiste de l'évolution sémantique », dans Candel Danielle \& Gaudin François (Dirs), Aspects diachroniques du vocabulaire, Mont-Saint-Aignan, Presses Universitaires de Rouen et du Havre, I5-88. 
Piguet Marie-France, 1996, Classe. Histoire du mot et genèse du concept, des physiocrates aux historiens de la Restauration, Lyon, Presses Universitaires de Lyon.

Pruvost Jean, 2002, Les dictionnaires de langue française, Paris, PUF (Coll. « Que sais-je ? », $\mathrm{n}^{\circ} 3622$ ).

Pruvost Jean, 2006, Les dictionnaires français : outils d'une langue et d'une culture, Paris, Ophrys (Coll. « Les essentiels »).

Rey Alain, 1977, Le lexique : images et modèles, Paris, Armand Colin.

Rey Alain, 1989, Révolution. Histoire d'un mot, Paris, Gallimard.

Rey Alain, 2011a, Dictionnaire amoureux des dictionnaires, Paris, Plon.

Rey Alain, 2orrb, La langue sous le joug, Mont-Saint-Aignan, Publications de l'Université de Rouen et du Havre, 52 p.

Rey Alain et al., 2007, Mille ans de langue française. Histoire d'une passion, Paris, Perrin. Robillard, Didier de \& Beniamino, Michel (Eds), 1993-1996, Le français dans l'espace francophone: Description linguistique et sociolinguistique de la francophonie, Paris, Champion.

Louis-Jean Rousseau, 2007, « La médiation linguistique : vers l'adaptation des principes méthodologiques et des pratiques terminographiques », réunion réseau Realiter, Bertinoro, Italie, 2007. http://www.realiter.net/wp-content/uploads/2013/07/ Louis-Jean_Rousseau.pdf.

Saussure (de) Ferdinand, 1985 , Cours de linguistique générale, édition critique de Tullio de Mauro, Paris, Payot.

Tamba-Mecz Irène, I991, La sémantique, Paris, PUF (Coll. « Que sais-je ? », n 655).

Teissier Claude, 2007, La poste : logique commerciale, logique de service public : la greffe culturelle, Paris, L'Harmattan.

Tevanian Pierre \& Tissot Sylvie, 2010, Les Mots sont importants, Paris, Libertali.

Thieltges Hélène, 2013, Distribution spatiale, stabilité et perception des dialectes chez deux espéces d'oiseaux guyanais (Cacicus cela et Cacicus haemorrhous), Thèse en biologie, Université de Rennes I. 


\section{D’une réflexion sur les dictionnaires électroniques}

\section{Introduction}

Se retrouver à Oxford, haut lieu historique et international de la culture, temple incontesté de la recherche universitaire, marqué par une architecture prestigieuse, et fonder son discours sur ce qui est « virtuel » a quelque chose d'étonnant. C'est pourtant bien la réalité : d'un côté des murs séculaires qui nous abritent et de l'autre un clavier et un écran, l'ordinateur, le règne du virtuel pour aller plus loin dans nos recherches. Sur la table de chêne au cœur de la bibliothèque, scintillent à foison les ordinateurs portables, signes palpables de l'agitation utile des neurones humains plongeant dans le cyberspace via les électrons. S'agissant de dictionnaires et de leur évolution, un mot vient immédiatement à l'esprit, la « métamorphose », et nous aimerions rapidement gloser et le mot et le concept pour mieux appréhender la révolution à laquelle nous assistons.

Métamorphose(s) ...

Métamorphose, c'est effectivement le mot qui vient spontanément, dans ce halo de sens qui se situe toujours « entre » les définitions comme l'affirmait Henri Meschonnic (2007), tant il est vrai que l'on ne s'exprime que bien rarement - en vérité, jamais - avec pour chaque mot un article de dictionnaire en tête, à la manière d'une machine qui irait figer le curseur sur le sens 3 ou le sens 5 ou encore 7 sagement répertoriés et cloisonnés 
par nécessité descriptive dans l'article de dictionnaire dévolu au mot prononcé. On commencera donc par analyser ce mot émergé de l'inconscient, métamorphose, pour évoquer l'avenir des dictionnaires de langue française.

Abordons donc ce mot et ce concept prometteur ou inquiétant : la métamorphose ... Et avant de l'appliquer aux dictionnaires dans leur évolution permanente, il n'est peut-être pas inutile de rappeler ce qu'en disent justement les dictionnaires. À commencer par le Dictionnaire françois de Richelet en I680, notre premier dictionnaire monolingue, qui de la « métamorphose » offre la définition suivante : «C’est le changement qui se fait par un Dieu, ou par une Déesse d'une personne en quelque autre forme ». Cette définition fait en réalité écho à une formule qui se disait encore au Grand Siècle pour désigner le paganisme, appelé « le temps de la métamorphose $\gg$.

Richelet offre du mot métamorphose quelques exemples éclairants : «Poëme qui contient quelque métamorphose. La métamorphose de Daphné en laurier. Ovide a fait quinze livres de métamorphoses. » Il est pertinent de citer Ovide, car le mot métamorphose est en effet né du titre francisé du poème d'Ovide, Les Métamorphoses, déjà évoqué en 1365 par Orseme, avant de devenir un nom commun attesté à la fin du XVe siècle. Quant au verbe métamorphoser, il s'agit, signale Richelet, d'une part de « changer une personne en une forme toute autre que celle que cette personne avoit » et d'autre part, au sens figuré et plus large, tout simplement de « changer ». Il faudra en fait attendre le début du XVIII siècle, notamment dans un mémoire de Réaumur destiné à « servir à l'histoire des insectes » publié en 1736 pour que la métamorphose relève de la vie animale, notamment du passage de la chenille au papillon.

Enfin, aujourd'hui la métamorphose reste tout d'abord le fait de changer de forme, de nature, au point de n'être plus reconnaissable, sont ainsi données en guise d'exemple dans le Grand Robert les métamorphoses de Vishnou, puis les métamorphoses des grenouilles ou du papillon, et enfin, de manière courante, le simple changement d'aspect d'un être ou d'un objet, tout en restant le même, par exemple les métamorphoses d'un acteur.

Autant de sens que nous retenons, mêlés, s'agissant des dictionnaires. Ainsi, la signification pour ainsi dire religieuse, mais aussi matérielle du dictionnaire, «le » dictionnaire qu'on consulte comme un oracle, par exemple 
l'in-folio de Robert Estienne, Thrésor de la langue françoise, consultable par les rares lecteurs érudits, a bel et bien subi une singulière métamorphose lorsque, aujourd'hui, dans le RER, à la faveur d'un thème recherché, on tapote sur une plaque de plastique de la taille d'un paquet de cigarette, pour se retrouver au cœur d'un dictionnaire tout entier, avec des images animées, information appelée d'un « ailleurs » cybernétique impalpable, consultable par tous les « connectés » de la planète, en vérité une masse incalculable.

Au-delà de ce fait brutal, quelles métamorphoses pourrions-nous signaler ? Il semble qu'elles pourraient être classées en diverses catégories, relevant d'abord de la conception même du dictionnaire et de sa fabrication, puis de sa réception et enfin de sa consultation très nouvelle. Et de là on pourra aussi imaginer des métamorphoses à venir.

Commençons par les métamorphoses perceptibles des dictionnaires dans leur conception, métamorphoses liées de très près à la fabrication. Ainsi, pas de véritable dictionnaire tant qu'on se trouve sur un rouleau de papyrus d'une quinzaine de mètres, lisible de manière linéaire mais pour ainsi dire ni recopiable ni consultable. On attend donc ce que techniquement on appelle le codex, pour que naisse un dictionnaire au sens réel du terme. Ce codex héritier des tablettes de bois, enduites de cire et attachées entre elles, en s'assimilant à un livre composé de feuilles offrant recto verso deux supports d'écriture, ouvre une ère moderne, en somme celle du livre pouvant être ouvert sur une table, recopié facilement, consultable à une page référencée par une table des matières ou par un ordre alphabétique : voilà qui donnera longue vie à la bible et au dictionnaire sur papier, sur parchemin, puis imprimé en petit nombre pour arriver jusquau livre de poche. Encore d'aujourd'hui pour ce dernier. Ou du gros dictionnaire en dix volumes. Qui ne se fabrique plus d'aujourd'hui. Le dernier est pour ainsi dire celui d'Alain Rey en quatre gros volumes, publié en $200 \mathrm{I}$.

De l'argile durcie à la plaque de plastique et ses scintillements en passant par le papyrus, le papier et son industrie, le support même des ouvrages de consultation a d'évidence considérablement évolué, passant paradoxalement du plus dur, la roche et l'argile, au plus fragile, le papier, mais protégé dans nos bibliothèque pour aboutir en définitive, au dématérialisé, transmis d'un bout à l'autre de la planète en quelques microsecondes, mais totalement dépendant de l'énergie électrique sans lesquels les supports informatiques 
sont lettre morte. Je peux lire encore à la bougie le Thrésor de Nicot de I606 que j’ai la chance de posséder, je pourrai lire longtemps encore le Dictionnaire culturel, je ne peux plus lire le Dictionnaire Zizomis de chez Hachette sur disquette souple, et je ne lirai rien de tous les grands dictionnaires sur cédérom ou sur Internet si je n'ai plus d'électricité. Un pouvoir immense certes mais aussi une fragilité immense.

Il reste à mesurer sur l'échelle chronologique la révolution électronique à laquelle on a assisté, au cours des dernières décennies. La première version sur cédérom du Grand Robert paraissait en 1989 et la deuxième en 2005, versions tirées du Grand Robert imprimé, mais offrant de nouvelles possibilités de consultation. En 1994, était publié le seizième et dernier volume du TLF sur papier; en 2000 , ce même dictionnaire, assorti d'un moteur de recherche, était consultable intégralement sur Internet ; en 2004, naissait le cédérom y correspondant. On bénéficiait alors pour un même texte de trois approches différentes selon le support choisi, papier, cyberespace ou cédérom. Dans le même temps, il était décidé que le Dictionnaire du Moyen français, conçu dans le sillage du TLF ne serait diffusé que sur support électronique. En 1996, était publié le Larousse multimédia encyclopédique, rejoignant la cohorte des encyclopédies multimédia, usant donc du texte allié à l'image et au son, dont la première, Encarta, avait été conçue par Microsoft quelques années plus tôt. En 1997, les Éditions Redon publiaient sur cédérom le Littré, et en 1999, les Éditions Champion emboîtaient le pas avec la première édition du Dictionnaire de l'Académie française. En 2005 , le Dictionnaire critique de Féraud était accessible sur Internet.

Un constat s'impose, au début du XXIe siècle : la panoplie des dictionnaires appréhendés à travers leurs supports distincts était pour le moins multiforme.

Deux remarques générales ne peuvent être évitées. La première concerne le fait que la fin du $\mathrm{XX}^{\mathrm{e}}$ siècle et le début du XXI' ${ }^{\mathrm{e}}$ siècle restent en très grande partie marquées par le seul transfert du contenu sur un nouveau support, électronique en l'occurrence. Le dictionnaire imprimé constitue en effet la matière même du dictionnaire installé sur cédérom, les deux produits continuant ensuite le plus souvent de coexister. D’une certaine manière, peu de dictionnaires sont actuellement conçus en dehors 
d'un référent imprimé. La voie est cependant ouverte. On rappellera ici les propos de L. Catach, directeur de la recherche informatique pour les dictionnaires Le Robert, propos tenus lors d'un colloque à l'Institut (2005): «À travers les éditions électroniques, nous avons la possibilité, au reste largement inexploitée, de commencer à nous affranchir de l'alphabet. » La dimension analogique est en effet désormais accessible et même vertigineuse dans ses possibilités : elle reste cependant pour ainsi dire inexploitée au début du XXIe siècle.

Une seconde remarque, presque banale, concerne l'aspect indéniablement révolutionnaire des supports électroniques qui, bien que liés à l'ordinateur et donc à une technologie en constante évolution, permettent dans la période de fin de siècle et de début de siècle de se déplacer avec le contenu des 16 volumes du TLF, des 6 volumes du Grand Robert, des différents grands dictionnaires du XVII ${ }^{\mathrm{e}}$ siècle, de l'Encyclopédie, etc., le tout accessible sur des cédéroms, dévédéroms ou sur le disque dur de l'ordinateur. Le truisme mérite d'être relevé : en moins de dix ans, grâce à l'espace électronique sans commune mesure avec l'espace limité du papier, une œuvre immense et jusque-là très difficile à rassembler, en permanence et en un même lieu, est désormais à portée de clavier. C'est encore plus vrai avec l'accessibilité presque partout à l'Internet.

Au-delà de ces évidences, s'impose le fait que le support électronique, en entraînant l'installation de balises informatiques dans le texte du dictionnaire, pour permettre toutes sortes de requêtes complexes, offre au chercheur des possibilités inespérées jusque dans les années 1990. On reprendra l'exemple offert par J.-M. Pierrel lors des démonstrations proposées pour le TLF informatisé, le TLFI. Ainsi, en quelques secondes, pouvoir procéder au recensement de tous les verbes ayant un sens particulier en marine et touchant au maniement des voiles, ou bien retrouver une citation de V. Hugo à partir seulement de quelques mots retenus, « $\mathrm{Ce}$ siècle avait ... », suffit à faire comprendre les possibilités nouvellement offertes qui font du dictionnaire un véritable corpus d'informations rapidement accessibles. En 2005, 200000 pages du TLFI étaient consultées par jour : le chiffre est suffisamment élevé pour faire comprendre que la consultation d'un dictionnaire sur support électronique devient une pratique usuelle. 


\section{Wikipédia ...}

Une autre aventure a commencé le Is janvier 200I. Il s'agit de Wikipédia, lancé pour soutenir Nupedia, projet de même nature mais uniquement écrit par des experts. Le succès fut fulgurant : en 20ro, ce sont des millions de pages et d'articles qui sont en effet rédigés par des centaines de milliers de bénévoles dans plus de 200 langues. Soulignons que Nupédia disposait de procédures de révision très solide, un comité scientifique, et des contributeurs hautement qualifiés.

Wiki désigne un logiciel, d'où wikipedia, sur le modèle d'Encyclope$d i a$, avec donc pour objectif une encyclopédie en source ouverte, collaborative, ouverte aux contributions des gens ordinaires. Il comptait déjà I000 articles le I2 février 200 r et 10000 autour de septembre, avec un taux de progression d'environ 1500 articles par mois. Le 30 août 2002, ils en sont déjà à 40000 articles. La première référence à un wikipédia en langue française date du 23 mars 2001 . En décembre 2002, fut créé Wiktionary avec pour objectif de produire un dictionnaire et un thésaurus des mots dans toutes les langues, en utilisant le même logiciel que Wikipedia.

En janvier 2004, on compte 200000 articles en langue anglaise, pour atteindre le 7 juin 2004300000 articles. En 2007, le calcul est astronomique: Wikipédia contient 7,5 millions d'articles dans environ 250 langues, et en 2008 se fête le ro millionième article sur Wikipedia. Quelques mois plus tard, il dépasse les 2,5 millions d'articles en anglais et en 2009 on atteint 3 millions d'articles.

La France a exercé une certaine résistance, avec ce qu'on a appelé un « militantisme anti-Wikipédia », mettant en doute la solidité des sources. Ainsi, un livre est écrit par cinq étudiants de Sciences Po sous la direction de Pierre Assouline (Gourdain et al 2007), pour critiquer les conséquences des principes éditoriaux de l'Encyclopédie libre. Une information fausse présentant P. Assouline comme champion de jeu de paume reste ainsi quelques mois avant d'être contestée.

Des actions en justice ont par ailleurs été intentées contre la Wikimédia Foundation à la suite d'insertion d'informations sur la vie privée et 
d'insertions fausses, mais les plaignants ont été déboutés par la justice le 29 octobre 2007, qui a considéré Wikipédia comme un hébergeur.

Voilà pour les faits. Un raz de marée d'informations. Gratuites. Et maintenant, une réflexion de fond qui s'impose et une sorte d'autocritique. Qui n'a pas en effet consulté Wikipédia ? Statistiquement personne. Plusieurs cas de figure se présentent de fait.

Le moins inquiétant correspond à la recherche rapide d'un renseignement factuel sur une date de naissance, la date d'un œuvre. Il y a certes des erreurs, mais bien des biographies sont néanmoins pratiques, qu'il s'agisse de personnages illustres ou tout simplement de notices biographiques. On est ici dans le cadre de la vérification rapide.

Inquiétant : une lecture sur un sujet que l'on ne connaît pas, une citation que l'on recherche, et qui bien souvent est tout à fait inexacte, non respectueuse de la forme et presque toujours sans référence.

Désopilant : si, dit-on, les articles de sciences dites dures, physique, mathématiques, informatique, etc., sont en général très fiables, parce qu'ils ne peuvent être écrits par des ignorants du sujet par définition très pointu, dans le domaine des Sciences humaines, on trouve plus souvent des interventions d'internautes qui s'imaginent avoir une vérité, à partir de toutes petites connaissances. Ils sont peu nombreux ceux qui inventeront une formule mathématique mais plus facile à trouver ceux qui imaginent avoir quelque chose à dire sur la littérature.

Révoltant : sitôt que l'on connaît un sujet, dans le domaine des sciences humaines, on constate les ravages du plagiat, des reprises de nos travaux sans être cités et l'invulnérabilité de Wikipedia : comment en effet s'attaquer à ce phénomène. On ne se bat pas contre un serveur ! Et on n'a pas le temps de corriger, en passant par des procédures chronophages.

Sidérant et éclairant : la satisfaction très largement marquée pour ce que propose Wikipédia repose sur un principe oublié : la prépondérance d'une réponse, sur la pertinence de la réponse que seul le spécialiste, celui-là même qui ne consultera pas l'article, peut évaluer. En somme qu'attend le lecteur sur un sujet qu'il interroge ? Une réponse immédiate, des éléments d'informations nombreux, des illustrations, des liens. Et cela, Wikipédia lui offre à profusion. Peu importe la véracité que de toute façon il ne peut vérifier. En définitive, la validité des informations est un problème exclu. 
Enfin Wikipédia asphyxie progressivement les grands dictionnaires patrimoniaux. Que le Petit Robert soit passé au-dessous des I00 000 exemplaires et que le Petit Larousse ne cavale plus à un million d'exemplaires comme en 200 I en sont les résultantes directes. Angoissantes pour qui sait la valeur et la validité testimoniale de ces deux ouvrages.

\section{Du meilleur outil, à ses limites : de nouveaux paramètres}

Quelques limites restent en effet à rappeler. Tout d'abord, il y a peu de chance que les dix mille dictionnaires édités depuis le XVI ${ }^{e}$ siècle soient un jour tous disponibles à portée de clavier. Ainsi, consulter cent ans du Petit Larousse, quarante ans du Petit Robert restera pendant longtemps encore le privilège de ceux qui ont pu physiquement les rassembler. Ensuite, la consultation comparée de plus de deux volumes distincts, par exemple la recherche de la définition d'un même mot à travers les six grands dictionnaires Larousse du XIX ${ }^{\mathrm{e}}$ au XXI ${ }^{\mathrm{e}}$ siècle - en supposant qu' ils soient un jour tous sur support électronique -, ou des différentes éditions du Grand Robert, restera quoi qu'il en soit difficile, faute d'écrans suffisants. Elle ne remplace pas pour l'heure la consultation de la série des volumes ouverts sur une large table.

Enfin, un problème n'est pas complètement réglé, celui de la conservation à très long terme de tout ce qui est offert sur support informatique. Le Trésor de Nicot de 1606 n'a guère vieilli sur papier : il n'est pas sûr que le produit informatique d'aujourd'hui sera conservable et conservé cinq siècles durant. Il devient presque impossible de lire aujourd'hui les premiers dictionnaires offerts sur disque souple parus en 1989. Le fait même qu'il y ait une claire prise de conscience de ces différents problèmes laisse néanmoins penser que les solutions viendront. Si demain Wikipédia ferme : quid des millions d'articles?

Rappelons que la progressive métamorphose électronique du dictionnaire s'inscrit dans la logique naturelle de l'évolution. Les dictionnaires sont en effet par nature des outils et, en tant que tels, les lexicographes ont 
depuis toujours su choisir le réceptacle le plus adapté. Une nouvelle révolution a indéniablement été apportée par l'informatique et par l'information accessible sur écran au bout du clavier. On ne peut douter du fait que, de la même manière que le passage du volumen au codex avait profondément modifié les esprits, il n'y ait déjà de nouveaux processus en cours dans la structuration des savoirs et de la pensée, ce qui ne peut que se traduire par de nouvelles mutations des outils de la langue et des savoirs que sont les dictionnaires.

Avec l'Internet, l'ère de la requête multidimensionnelle et analogique est ouverte, et les effets à rebours sur la dictionnairique, c'est-à-dire la mise en forme éditoriale en somme, et la lexicographie, la recherche sur les mots, est décisive, car somme toute, les produits dictionnairiques à élaborer sont toujours dépendants du type de demande. Et, de fait, le nouvel utilisateur de dictionnaires est devenu très exigeant : le virtuel lui a donné en effet le goût d'une requête infinie.

Quels sont ces nouveaux paramètres?

Une première conséquence est l'élargissement du code définitoire. Le langage écrit et oral de type linéaire reste certes le code initial et le plus souvent directeur, mais il ne suffit plus. Ainsi, le public petit à petit métamorphosé en poseur de requêtes, plus ou moins explosives, en nouveau conquérant de l'informatique, ne supporterait plus qu'un support électronique, dont on sait qu'il constitue un forme d'inscription unique et homogène pour l'écrit, l'image et le son, n'offre qu'une définition textuelle pour des mots comme « guitare », « cigale », « menuet », « trille », etc. Définir une « éclipse » ou une « écluse » sans animation visuelle parâ̂trait également tout aussi insuffisant. L'absence d'illustrations sonores et visuelles est dorénavant considérée comme une lacune sensible de l'outil proposé, une lacune que s'empressera évidemment de combler la concurrence, cette dernière demeurant un puissant stimulant de la dictionnairique. Le multimédia fait désormais partie des modes d'expression de la définition.

Une seconde conséquence est celle qui substitue au paramètre de l'espace typographique le paramètre du temps. La nouvelle dimension n'est plus en effet celle de l'espace d'inscription très limité dans le dictionnaire papier, mais celle de l'espace illimité offert à l'information. Et par conséquent la nouvelle mesure prépondérante devient celle de l'accès à l'information 
pour le diffuseur, et celle du temps possible de consultation pour l'utilisateur. Calibrer la demande en fonction non pas du nombre de pages, mais du temps que l'on a de disponible, devient nécessaire. Certains sujets sont si riches d'informations sur Internet que le caractère pléthorique des documents peut rendre tout à fait impossible la consultation de l'ensemble dans un délai raisonnable. Elisabeth Badinter interrogée dans un article du Monde du I3 juin 1998, souligne que le temps de recherche est considérablement allongé : quand l'ensemble des documents sera rassemblé sur l'ordinateur « on sera submergé par la masse de l'information. Plus qu'une tentation, il y aura presque une obligation de tout lire. Cela rendra la recherche plus longue, voire impossible, par démangeaison de l'exhaustivité » (De Roux, Emmanuel, Van Renterghem Marion : 1998).

L'information offerte presque en temps réel devient par ailleurs la règle exigée par l'utilisateur. Du côté dictionnairique, l'encyclopédie constituée d'articles figés, enfermés dans un objet, est déjà dépassée : sans mise à jour régulière des articles par une équipe rédactionnelle toujours disponible, sans liens Internet qui le permettent, l'encyclopédie n'a aucune chance de survivre. Quant au dictionnaire de langue, on supportera mal de ne pas bénéficier directement des dernières analyses conduites dans le domaine de la recherche. Les dictionnairistes ont donc, entre autres, pour mission, d'imaginer les structures performantes qui répondent aux requêtes des utilisateurs, et les lexicographes seront amenés à davantage encore observer en continu l'évolution de la langue. Leur point de vue sera de plus en plus sollicité par le grand public et il est clair qu'au bout de l'hypertexte, il souhaite se trouver en prise directe avec la recherche. D'une certaine manière, l'alcôve scientifique et le laboratoire en arrière du magasin, jusque-là inaccessibles, deviennent territoires porte ouverte.

Une troisième conséquence est la fragmentation réticulée de l'information sans la garantie d'une validation. En effet, les navigateurs d'Internet ont déjà fait de la toile, par le biais des moteurs de recherche, une encyclopédie fragmentée et aléatoire. Il est de fait qu'il n'est guère de sujet lancé dans le cyberespace qui ne trouve de réponses sur tel ou tel site, et souvent de manière surabondante. Mais cette encyclopédie polymorphe que représente la toile fait courir le danger propre à toute liberté sans validation ; sans communauté d'experts pour cautionner le savoir, celui-ci reste aléatoire, 
révolutionnaire, pertinent ou fallacieux, et en tout cas sans garantie. Il est effectivement possible à quiconque actuellement de s'improviser lexicographe et de rédiger sur la toile un dictionnaire exécrable qu'il offrira à un internaute ainsi subverti à son insu. Contrairement à cette diffusion incontrôlée, la maison d'édition patentée reste, par essence, responsable de ses publications, et pour maintenir les secteurs qu'elle garantit en termes de savoir, il lui faut donc s' installer dans le paysage pour occuper un espace du réseau que la consultation désordonnée et hasardeuse des « surfeurs » ne pourra éviter. Sous peine d'être en partie marginalisée par la consultation « sauvage », les maisons d'édition spécialisées dans l'élaboration des dictionnaires doivent investir la toile, et trouver les formules qui ne laissent pas les navigateurs égarés loin des îlots de sécurité que représentent les maison d'édition ou en tout cas loin de toute formule contrôlée par un groupe. Le grand décloisonnement dans un « village global » de la lexicographie est en marche.

\section{Les dictionnaires de spécialité}

Il se trouve qu'en acceptant la direction éditoriale de la maison d'édition Honoré Champion, j’ai souhaité ouvrir au passage deux collections portant sur les dictionnaires, collection destinée au grand public cultivé et non au public de spécialistes.

La première collection, Champion les mots, est en quelque sorte métalexicographique, elle porte sur un thème - le vin, le loup, le jardin, le citoyen, l'élection, le chat, le cirque, etc. - étudié à travers notre patrimoine lexicographique, en gros de Robert Estienne aux derniers Petit Robert et Petit Larousse illustré, en passant par les dictionnaires des XVII ${ }^{e}, \mathrm{XVIII}^{\mathrm{e}}$ et $\mathrm{XIX}^{\mathrm{e}}$ siècles, pour aboutir aux dictionnaires relativement récents, par exemple le $T L F$ et Dictionnaire culturel d'Alain Rey. Je suis en l'occurrence très surpris de deux choses à propos de cette collection : en ayant l'occasion d'être invité à diverses émissions radiophoniques ou autres, pour ce type d'ouvrages qui certes offre des définitions à travers les siècles, des 
informations issues des dictionnaires, mais qui reste cependant un texte chronologiquement rédigé, deux fois sur trois on évoque un dictionnaire. Il s'agit de mots, c'est donc un « dictionnaire » aux yeux des journalistes. J'y vois un statut en évolution : l'ordre alphabétique n'est plus une condition sine qua non. La seconde surprise, heureuse, est le plaisir qu'y prennent les lecteurs érudits comme les lecteurs que seul le thème intéresse : la connaissance des dictionnaires d'hier intéresse tout le monde.

La seconde collection s'appelle explicitement Dictionnaire, donc là pas d'erreur d'interprétation. Et de fait, toute spécialité y a sa place dès lors qu'elle est traitée par un spécialiste et que les informations y sont offertes dans l'ordre alphabétique et de manière rigoureuse. Dictionnaire du rugby, Dictionnaire des écrivains francophones classiques, du Nord, du Sud, Dictionnaire du désir de lire, Dictionnaire de la bonne chère au XVIII e siècle, etc. Et là aussi un constat s'impose : il est croissant le nombre de spécialistes qui souhaitent s'inscrire dans ce mode de diffusion du savoir à la fois fragmenté et cohérent, cloisonné tout en étant décloisonné grâce aux renvois. Or, indéniablement le public est au rendez-vous malgré les faibles moyens de communication d'une maison comme Honoré Champion, ne fonctionnant que sur catalogue et peu représentée jusque-là dans les librairies.

Au moment où l'édition est en crise, de fait, on le constate, les dictionnaires spécialisés restent très courus et se développent. D’où cette idée d'un envol ...

\section{Conclusions}

Pour conclure, il faut admettre que le dictionnaire est tributaire du temps qui passe et que c'est en très peu de temps que se vivent les évolutions décisives à la fois riches et lourdes de conséquences. À l'échelle d'une vie ordinaire comme la mienne, la seconde moitié du $\mathrm{XX}^{\mathrm{e}}$ siècle, et cette première décennie du XXIe, j'ai d'abord recopié des vieux dictionnaires qu'on me prêtait, faute de photocopieuses, puis très rapidement, pour ce diplôme petit à petit oublié que représentait une maîtrise sous la direction 
de Bernard Quemada, j’ai travaillé sur un dictionnaire onomasiologique avec des cartes perforées, Io o०० à l'époque, et une impression sur grandes feuilles en accordéon. Vint la thèse, frappée sur une machine à écrire avec un double sur papier pelure, un matériau pour ainsi dire disparu, puis ce fut l'avènement de l'ordinateur, Alain Rey se souvient d'un ordinateur qui m'avait été prêté chez Robert, un engin impressionnant, avec disquettes souples, 5 pouces .... les dictionnaires qui ont été vendus ainsi (Zizomis) ne sont plus lisibles, les lecteurs ont d'ailleurs disparu. Ce fut ensuite l'ère des disquettes, elles-mêmes pour ainsi dire disparues, et nous en sommes aux clés USB. Viendra sans doute très vite un autre processus. Pour l'heure, règne le « virtuel » du cyberspace via Internet.

On ne peut alors s'empêcher de penser à Victor Hugo, s'exclamant dans Les Rayons et les Ombres, « Que peu de temps suffit pour changer toutes choses! Nature au front serein, comme vous oubliez! Et comme vous brisez dans vos métamorphoses Les fils mystérieux où nos cœurs sont liés. » Et dans le même élan, mais tourné vers l'avenir, on pourrait aussi citer Romain Rolland s'interrogeant ainsi dans les Musiciens d'autrefois (1903): « L'art meurt-il jamais ? Il se métamorphose, il s'adapte aux circonstances. »

Eh bien, il en va de même des dictionnaires. Ils s'adapteront ! Et Oxford restera un haut lieu de savoirs, des manuscrits aux imprimés en passant par les électrons. En attendant peut-être d'autres révolutions encore.

\section{Références}

De Roux Emmanuel \& Van Renterghem Marion, 1998, « Chercheurs et écrivains face au virtuel ; Lébauche d'une vaste bibliothèque virtuelle de dimension planétaire, évoquée lors d'un colloque organisé par la Bibliothèque nationale de France, risque de modifier les conditions de la recherche comme celles de l'écriture. Et de brouiller les frontières entre les métiers du livre », in Le Monde 13 juin.

Dictionnaire de l'Académie française, 1999, Paris, Champion.

Dictionnaire du Moyen Français (1330-ISOo) disponible sur : <http://www.atilfffr/ $\mathrm{dmf} />$

Estienne Robert, 1549, Thrésor de la langue françoise, Paris, impr. de R. Estienne. 
Féraud Jean-François, Le Dictionnaire critique de la langue française informatisé et son supplément manuscrit en mode image 1787-1807, <http://www.cnrtl.fr/dictionnaires/anciens/feraud/index.php>

Gourdain Pierre, O'Kelly-Florence, Roman-Amat-Béatrice et Soulas-Delphine \& Von Droste zu Hülshoff Tassilo, 2007, La Révolution Wikipédia : Les encyclopédies vont-elles mourir?, Paris, Mille et une Nuits.

Hugo Victor, I840, CEuvres complètes de Victor Hugo, Poésie VII, Les Rayons et les Ombres, Paris, Delloye, Libraire.

Larousse multimédia encyclopédique, 1996, Paris, Larousse.

Le Grand Robert, 1989, Paris, Le Robert.

Le Littré, Dictionnaire, CD Rom E, 1999, Paris, Éditions Redon.

Meschonnic Henri, 2007, Ethique et politique du traduire, Lagrasse, Editions Verdier.

Nicot Jean, 1606, Thresor de la Langue Françoyse, tant Ancienne que Moderne, Paris, David Douceur.

Pierrel Jean-Marie, La préface du TLFi, <http://www.atilf.fr/IMG/pdf/La_preface_du_TLFi_par_Jean.pdf>

Réaumur René Antoine de, 1734-1742, Mémoires pour servir à l'histoire des insectes, Paris, de l'Imprimerie Royale.

Rey Alain, 200I, Dictionnaire culturel en langue française, Paris, Le Robert.

Richelet César-Pierre, Dictionnaire françois, contenant les mots et les choses, Genève, Jean Herman Widerhold.

Rolland Romain, 1903, Musiciens d'autrefois, Paris, Hachette.

Wolowski, Louis François Michel Raymond (Eds), 1864, Oresme Nicolas, 1365, Traité de l'origine, de la nature, du droit et des altérations de la monnaie, Paris, Librairie de Guillaumin et Cie.

Zizomis dictionnaire électronique, 1996, CD ROM PC, Paris, Hachette. 


\section{Les mots complexes en français contemporain : pour qui n'a pas grandi avec le « Dinotrain »}

Les mots complexes comme « fleur horloge » ou « Dinotrain » sont à l'articulation du lexique et de la syntaxe (Arnaud et Renner 2014). Longtemps négligés ou peu décrits autrement que comme « mots composés », catégorie à géométrie variable puisque pour certains linguistes au moins elle comprend aussi des noms modifiés par un adjectif ou par un syntagme prépositionnel (Riegel et al. 2009: 912), les mots complexes font l'objet d'un regain d'intérêt, en tant que créations lexicales permettant de véhiculer de nouvelles significations. Les travaux récents analysent les aspects morphologiques, mais aussi sémantiques de ces créations, dans une perspective synchronique ou diachronique, et souvent translinguistique (Lieber \& Stekauer 2009, Scalise $\&$ Vogel 20I0). La psycholinguistique développementale s’intéresse également à ces unités étonnamment proches des premières combinaisons à deux termes que produisent tous les enfants du monde (voir par exemple François et al. 1977 ; Tomasello \& Brooks I999), et qui peuvent être assimilées à une ébauche de syntaxe. Prenant acte de travaux portant sur l'acquisition d'une langue maternelle ou de langues étrangères, mais aussi sur les troubles du langage comme l'aphasie, Jackendoff (1999) voit dans la composition un principe primitif (cité in Malmkjaer 2010 : 318). Après Bickerton (1990), pour qui les langues modernes constituaient des évolutions sur la base d'un substrat, ou protolangage, Jackendoff(1999:276) analyse en effet les composés de la langue anglaise comme d'éventuels « fossiles » de ce protolangage.

I Les données présentées dans cet article sont issues d'un travail en collaboration avec Susanne Borgwaldt (Université de Siegen, Allemagne) et Vincent Renner (Université Lumière Lyon 2 et CRTT). Je remercie Vincent Renner pour son aide précieuse. Je remercie aussi l'école élémentaire Bizanet de Grenoble, ainsi que Florence Durand qui a bien voulu m’accueillir dans sa classe. 
La plupart des travaux portent sur les langues germaniques (et en grande majorité sur l'anglais) mais la présence de tels composés en français, si elle est reconnue depuis la fin du $19^{\text {ème }}$ siècle $^{2}$, reste assez peu étudiée, à part dans quelques travaux récents qui abordent la question dans une perspective lexicologique et terminologique (voir par exemple Arnaud 2003 ; Arnaud et Renner 2014). En psycholinguistique, cette question a principalement été abordée comparativement, pour voir si des différences de traitement des mots composés pouvaient être observées chez les enfants francophones (voir par exemple Nicoladis \& Krott 2007). Les hypothèses développementales reposent sur le repérage de différences structurelles entre les langues germaniques et les langues romanes : la fréquence d'utilisation des procédés de composition à l'œuvre dans les mots composés est considérablement moins importante en français, où l'on utilise plus couramment des syntagmes prépositionnels. Pour reprendre un exemple de Nicoladis (2003: 320), là où l'anglais utilise coffee cup, le français utilise tasse à café.

Nous présentons ici les résultats d'une expérience pilote en classe de CE2-CMr, qui permettent de décrire et d'analyser la préférence des locuteurs pour la composition lorsqu'il s'agit de nommer des objets hybrides.

\section{Les mots complexes en français}

Nous distinguons les unités lexicales simples (mots simples, ou monomorphémiques) des unités lexicales complexes (mots complexes). Ces derniers peuvent être construits ou non-construits, au sens où leur structure interne n'est pas toujours lisible d'un point de vue morphologique et sémantique : «Un mot construit est un mot dont le sens prédictible est entièrement compositionnel par rapport à la structure interne »(Corbin I987:6). L'inclusion des mots non-construits dans les unités complexes permet

2 Voir par exemple le Traité de la formation des mots composés de la langue française comparée aux autres langues et au latin d'Arsène Darmesteter. 
d'intégrer des « unités hybrides », dans lesquelles au moins « un segment ne correspond pas à une unité morphologiquement identifiable » (Petit 2009 : 36). Nous préférons parler de mots complexes plutôt que de mots composés, en raison de la variété des définitions de ce dernier terme, mais la complexité des unités que nous allons décrire résulte bien de la composition.

Par composition, nous entendons l'enchaînement ou l'enchâssement d'au moins deux items lexicaux en vue de former un nouvel item lexical, dont le sens est généralement dérivé de celui des items qui le composent (Borgwaldt \& Benczes 20II : 222) sans être nécessairement toujours compositionnel. Nous ne faisons pas d'hypothèses sur le fait que la composition mette en jeu ou non une compétence grammaticale.

Certains auteurs distinguent les noms composés des suites de deux noms (ou « suites NN », Arnaud 2003:19 ${ }^{3}$ ) : qu'elles constituent ou non une classe à part, ces suites intéressent notre étude à plus d'un titre. Il faut en effet remarquer, avec Arnaud (ibid.), le recours fréquent au style télégraphique, qui implique la suppression des mots de classe fermée (prépositions et, le cas échéant, déterminants), sur des pancartes, catalogues et autres supports de communication publicitaire. Du fait de leur usage sur de tels supports, ces suites « se rencontrent non pas insérées dans des énoncés à prédicat verbal mais hors syntaxe, isolées sur des panneaux, des emballages » (Arnaud 2003:20). Dans le dispositif expérimental que nous décrivons ci-dessous, nous demandons aux enfants de nommer des objets hybrides et le résultat est également une dénomination produite hors syntaxe. Arnaud remarque également qu’en diachronie, ces composés « timbreposte » étaient rares jusqu’à la fin du I $9^{\text {ème }}$ siècle (Arnaud 2003: II9-I3I). Distincts des composés dits « attributifs », comme ville champignon ou chapeau melon, ces suites de deux noms se caractérisent en effet par « une relation de détermination non-analogique du premier nom par le second » (ibid.), que le français exprime également (et parfois uniquement) à l'aide d'un syntagme prépositionnel. Que l'on dise tasse à café (et non *tasse café) ou moule à gaufres (et non *moule gaufres), alors que l'on parle désormais

3 Arnaud cite notamment, outre timbre-poste - sortie piétons (sortie pour piétons), accès gare (accès à la gare) ou numéro client (numéro de client). 
plus facilement de menu enfant que de menu pour enfant témoigne donc probablement de l'existence de contraintes qui rendent possible, ou non, l'effacement de la préposition : la relation contenant-contenu semble rendre l'usage de la préposition obligatoire.

L'usage de mots-valises comme flaquastrophe ou prioratét constitue également une tendance du discours publicitaire : ils sont au cœur des « jeux de langue auxquels se livrent publicitaires, journalistes et politiciens soucieux de retenir l'attention de leur public » (Muller 1986:203). Procédés littéraires remontant à Rabelais et même Aristophane (ibid.), les mots-valises ont aujourd'hui un usage beaucoup plus large, de la publicité au discours spécialisé : dans un monde où la nouveauté est le maître mot, ils sont devenus un moyen privilégié pour nommer des référents nouveaux. Mais si les mots-valises «participe[nt] aux procédés de création néologique du français contemporain » (Reboul 1996), ils n'en restent pas moins des « productions extra-grammaticales [résultant] de manipulations sophistiquées et tout à fait conscientes » (Fradin et al. 2009): il n'y a pas à proprement parler de patron de formation des mots-valises, si bien que les relations pouvant exister entre la forme des mots-valises et leur interprétation sont difficiles à cerner.

\section{Une expérience pilote en classe de $\mathrm{CE}_{2}-\mathrm{CM}_{\mathrm{I}}$}

L'expérience dont nous présentons ici la mise en place utilise un paradigme expérimental original, créé par Borgwaldt \& Benczes (20II) pour des travaux sur le hongrois et l'allemand (ibid.) puis sur l'ukrainien (Borgwaldt

4 Nous reprenons deux des mots-valises cités par Bonhomme et Horak (2009 : 54): ce sont des slogans utilisés par la société suisse Zurich Assurances, en lien avec des images qui représentent les risques couverts par la compagnie. Flaquastrophe est associé à une paire de bottes à moitié recouverte d'eau, et prioraté à un triangle de danger qui apparaît entouré de bris de verre sur une route. 
et al. 20I2). Il s'agit d'une tâche de dénomination non contrainte, qui permet non pas de tester la compréhension et la production de nouveaux composés, comme l'ont fait de nombreuses études psycholinguistiques depuis les années 1980 (et les travaux portaient en majorité sur des langues germaniques et sur l'hébreu), mais d'analyser les stratégies préférées par les locuteurs interrogés lorsqu'on leur demande de former de nouveaux mots pour nommer des objets hybrides.

Il existe plusieurs tournures possibles pour nommer des objets hybrides comme ceux que nous avons montrés aux enfants ${ }^{5}$ : nous avons utilisé des montages photographiques représentant par exemple une tente faite en peau de pomme, ou encore un avion dont le fuselage a été remplacé par une banane.
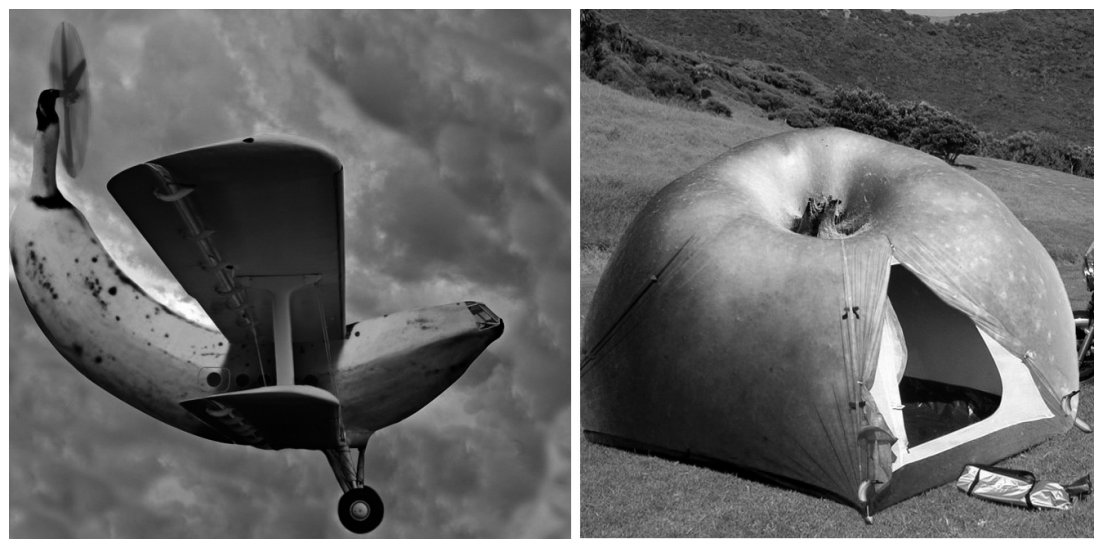

Image I : deux exemples d'objets hybrides (source : Freakingnews.com)

Nous distinguons ${ }^{6}$ la composition de mots complexes (qui correspond à la dénomination de l'objet hybride, voir exemples i et 2 ci-dessous) de la description par une expression plus longue qui correspondra souvent en français à un nom tête complété par un syntagme prépositionnel ou par une

5 Toutes les images sont celles qui ont été sélectionnées et utilisées par Borgwaldt et al., elles proviennent du site FreakingNews.com.

6 Voir notamment Bücking (2010). 
relative (comme dans l'exemple 3). L'utilisation d'un syntagme prépositionnel associant seulement une préposition et un nom nu (comme dans l'exemple 4) est plus compacte et probablement plus proche des mots complexes ci-dessus. Les syntagmes nominaux associant un nom et un adjectif sont également possibles, comme dans l'exemple 5 . Mais dans l'un et l'autre cas, ces usages ne constituent pas des dénominations au sens où l'entend Riegel (I99I), ils produisent plutôt une description (voir aussi Renner 2006 : 2I)

(i) Une fleur-horloge, un avion-banane

(2) Une florloge, une banavion

(3) Une horloge dans une fleur, une banane qui a des ailes et une hélice

(4) Une horloge en fleur, un avion en banane

(s) Une horloge fleurie, une banane ailée

Les enfants de maternelle que nous avons interrogés lors d'un prétest n'ont produit que très peu de mots complexes, et leurs productions correspondaient en majorité à des descriptions. Certains travaux (notamment Clark I998) suggèrent que parce que les noms composés sont peu fréquents et assez peu productifs en français, les francophones choisiraient plus rarement les composés dans des tâches de création lexicale, et ce jusqu’à l'âge de 7 ans au moins. Il ne s'agissait pas pour nous de tester l'hypothèse d'un développement plus tardifde ces éléments chez les enfants francophones. Pour évaluer la place relative de chacune des stratégies de dénomination possibles chez des enfants d'âge scolaire, nous avons choisi d'effectuer l'expérience pilote en classe de $\mathrm{CE}_{2}$-CMI, c'est-à-dire avec des enfants dont lâge est généralement compris entre 8 et 9 ans. L'école comporte une section internationale espagnole, mais la plupart des enfants interrogés étaient soit des francophones monolingues, soit des enfants présentant un bilinguisme récessif, car le français reste pour tous la langue de scolarisation et de socialisation (l'enseignement en langue espagnole ne se faisant quà raison d'une heure par jour pour les enfants de la section espagnole). De plus, il n'existe pas à notre connaissance de différences typologiques entre le français et l'espagnol qui pourraient motiver l'emploi de patrons empruntés à l'espagnol. Nous avons donc uniquement exclu les réponses d'un enfant arrivé d'Espagne depuis quelques mois seulement, qui a produit une grande majorité de descriptions du type « le serpent se transforme en banane » ou « dans le ciel il y a un kangourou ». 
Après deux images permettant de familiariser les enfants avec la tâche, nous leur avons proposé 40 images d'objets hybrides, qui représentent des mélanges de deux animaux (19 images) ou d'autres objets de la vie quotidienne. Nous avons choisi des référents simples et évocateurs pour éviter les problèmes de lecture des images chez des enfants encore jeunes. Les images qui ont le plus souvent posé problème ${ }^{7}$ sont d'ailleurs celles qui représentaient des relations plus abstraites, en particulier celles qui combinaient la forme d'un objet ou animal avec la matière d'un autre, comme par exemple des objets ayant la matière d'une pomme et la forme d'un cœur, la matière d'un nuage et la forme d'un kangourou (voir la partie droite de l'image 2 ci-dessous), ou encore la matière d'une orange et la forme d'un cube. D'autre part, les images présentées aux enfants ne contenaient pas de relation contenant-contenu, à l'exception peut-être d'une image composée d'un cornet de glace surmonté d'un cactus (image 2, partie gauche), qui pourrait représenter un cornet de cactus. Cependant, aucun des enfants n'a choisi de le nommer ainsi, et leurs choix suggèrent que l'image évoque avant tout une glace : or il n'y a plus dans glace au cactus de relation contenantcontenu (pas plus que dans glace-cactus ou glactus).
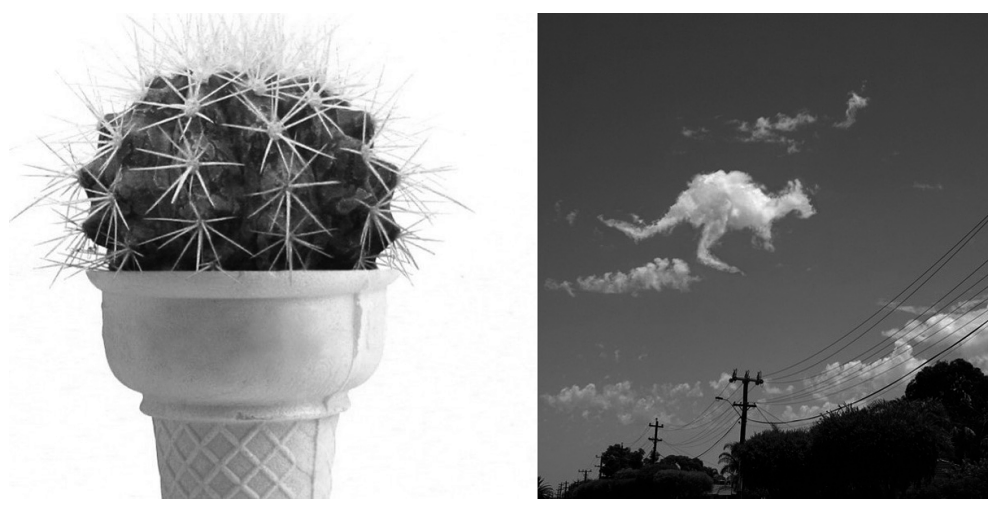

Image 2 : exemple d'hybrides représentant des relations contenant-contenu, et forme-matière

7 Etonnement et questions du type « c'est quoi ? », entrainnant des temps de production plus longs ou une absence de réponse. 
Enfin, nous avons délibérément choisi des mélanges équilibrés (sans différence de saillance) de chacun des deux éléments représentés sur l'image, car nous n'avions pas d'hypothèses sur le choix de l'un ou l'autre élément comme nom tête, et préférions favoriser la plus grande variété de réponses possible.

\section{Premiers éléments d'analyse : résultats quantitatifs}

Les résultats ci-dessous concernent les réponses de 23 enfants, qui étaient en majorité en classe de $\mathrm{CE}_{2}$ (6 $\mathrm{CM}_{1}$ et $17 \mathrm{CE}_{2}$ ). Les données pour chaque enfant portent sur 40 images différentes : il y a donc 40 propositions de dénomination par sujet, soit au total 920 éléments analysés.

Tableau I : structure morphologique des réponses des 23 enfants

\begin{tabular}{|l|c|c|}
\hline \multicolumn{1}{|c}{ Patron } & Nombre de productions concernées & Pourcentage \\
\hline NN & 4 I 7 & $45 \%$ \\
\hline Mots-valises & 260 & $28 \%$ \\
\hline N + PP & I 36 & I $5 \%$ \\
\hline N + Adj & 4 I & $4 \%$ \\
\hline Autres & 5I & $6 \%$ \\
\hline Incomplet & I 5 & $2 \%$ \\
\hline
\end{tabular}

Dans l'ensemble, les enfants ont donc préféré le patron NN, puis les mots-valises. Le recours à des syntagmes prépositionnels représente moins d'un sixième des données, ce qui semble aller contre l'idée (fréquente dans les travaux qui portent sur l'acquisition du français dans une perspective typologique, cf. supra) qu'ils constituent une stratégie plus répandue et productive que la composition dans la langue française. Il en va de même, et à plus forte raison encore, pour les combinaisons d'un nom et d'un adjectif, qui ne représentent que $4 \%$ de l'ensemble. Cependant, ces préférences correspondent 
aussi au type de tâche concernée ${ }^{8}$ : la présentation d'images fabriquées dont on demande une dénomination libre, produite hors contexte et hors syntaxe favorise la création de néologismes. De plus, le recours à un syntagme prépositionnel ou à un adjectif produit des effets de sens différents : dans les deux cas, l'objet est décrit plus qu'il n'est nommé.

Si les résultats quantitatifs des seuls élèves de CMr ne constituent pas un ensemble suffisant pour que nous puissions les comparer à ceux des $\mathrm{CE}_{2}$, il apparaît cependant que les trois quarts des productions des CMr sont des mots-valises, alors que chez les $\mathrm{CE}_{2}$ ils ne correspondent quà I $2 \%$ de l'ensemble. Ces différences éclairent le tableau général, au sein duquel le pourcentage de mots-valises semble particulièrement élevé. En effet, s'ils sont mis en avant dans certains discours spécialisés, les mots-valises restent assez peu fréquents dans la langue.

Le fait que les $\mathrm{CE}_{2}$ soient presque trois fois plus nombreux que les CMr doit évidemment être pris en considération, surtout s'il s'avère que les stratégies varient plus fortement d'un individu à l'autre qu'au sein d'une même passation (cf. infra, résultats qualitatifs).

Tableau 2 : Structure morphologique des réponses des 6 enfants de CMI

\begin{tabular}{|c|c|c|}
\hline Patron & Nombre de productions concernées & Pourcentage \\
\hline NN & I 8 & $8 \%$ \\
\hline Mots-valises & $\mathrm{I} 76$ & $74 \%$ \\
\hline N + PP & 35 & $14 \%$ \\
\hline N + Adj & 3 & I $\%$ \\
\hline Autres & 2 & I $\%$ \\
\hline Incomplet & 5 & $2 \%$ \\
\hline
\end{tabular}

Cependant, il semble bien que la production de mots-valises soit plus complexe sur le plan cognitif, notamment en raison de la nécessité de choisir entre plusieurs combinaisons possibles (Borgwaldt et al. 2012:82). Nous

8 cf. Borgwaldt et al. (2012) qui font remarquer l'originalité du paradigme expérimental. 
n'avons pas mesuré les temps de production pour chaque item, mais nous avons associé à chaque passation individuelle sa durée d'enregistrement, c'est-à-dire la durée globale de production des 42 dénominations (prétest inclus). La comparaison des durées moyennes représentée dans le Tableau 3, ci-dessous, montre une augmentation assez netteme avec le nombre de mots-valises produits.

Tableau 3 : Durées de passation

\begin{tabular}{|c|c|c|}
\hline Sujets & $\begin{array}{c}\text { Durée moyenne en } \\
\text { secondes }\end{array}$ & $\begin{array}{c}\text { Durée moyenne } \\
\text { approximative }\end{array}$ \\
\hline TOTAL & 459 & 8 minutes \\
\hline CE2 & 435 & 7 minutes \\
\hline CMI & 518 & 8 minutes 30 \\
\hline $\begin{array}{c}\text { N'ayant pas produit de } \\
\text { mots-valises }\end{array}$ & 394 & 6 minutes 30 \\
\hline $\begin{array}{c}\text { Ayant produit plus de 20 } \\
\text { mots-valises }\end{array}$ & 572 & 9 minutes 30 \\
\hline
\end{tabular}

A titre d'exemple, l'image 2 ci-dessus a donné lieu aux mots-valises suivants : avionba, bananevion ou bananavion, mais aussi banavion, avebane, avibanane et bananair. Les patrons NN, quant à eux, varient beaucoup moins, puisque les seules propositions sur ce même item ont été : une banane avion et un avion banane. L'hypothèse d'une progression développementale des composés aux mots-valises mériterait donc probablement d’être examinée.

\section{Résultats qualitatifs}

L'analyse individuelle des résultats suggère que les enfants n'ont pas élaboré ces noms d'objets et d'animaux au hasard. Le choix d'une stratégie de dénomination se dessine assez nettement chez presque tous les enfants, et ces choix sont, au moins chez les $\mathrm{CE}_{2}$, basés sur « les schémas de formation lexicale 
de la langue ${ }^{9} \gg$ comme le suggère Clark (198I : 299) à partir des productions d'enfants plus jeunes. En effet, I2 des 17 enfants de CE2 ont utilisé la combinaison ou la juxtaposition de deux noms, et le recours aux syntagmes prépositionnels constitue également une stratégie majoritaire chez 5 enfants.

Tableau 4 : Analyse des stratégies préférées par chacun des 23 sujets

\begin{tabular}{|c|c|c|c|}
\hline Sujets & $\begin{array}{c}\text { Nombre de } \\
\text { stratégies } \\
\text { différentes utilisés }\end{array}$ & $\begin{array}{l}\text { Nombre d'éléments } \\
\text { correspondant à la } \\
\text { stratégie majoritaire }\end{array}$ & $\begin{array}{l}\text { Stratégie } \\
\text { majoritaire }\end{array}$ \\
\hline CMI enfant I & 5 & $2 I$ & $N+P P$ \\
\hline CMI enfant 2 & 3 & 37 & Mots-valises \\
\hline CMI enfant 3 & 3 & 38 & Mots-valises \\
\hline CMI enfant 4 & 4 & 26 & Mots-valises \\
\hline CMI enfant 5 & 2 & 39 & Mots-valises \\
\hline CMI enfant 6 & 2 & 37 & Mots-valises \\
\hline$C_{2}$ enfant I & 2 & 28 & NN \\
\hline CE2 enfant 2 & 2 & 37 & NN \\
\hline $\mathrm{CE}_{2}$ enfant 3 & 4 & 26 & $N+P P$ \\
\hline $\mathrm{CE}_{2}$ enfant 4 & 3 & 30 & NN \\
\hline $\mathrm{CE}_{2}$ enfant 5 & 5 & 20 & $\mathrm{~N}+\mathrm{PP}$ \\
\hline $\mathrm{CE}_{2}$ enfant 6 & 4 & 37 & NN \\
\hline $\mathrm{CE}_{2}$ enfant 7 & 4 & 33 & NN \\
\hline $\mathrm{CE}_{2}$ enfant 8 & 3 & 33 & NN \\
\hline $\mathrm{CE}_{2}$ enfant 9 & 3 & $3 \mathrm{I}$ & $N+P P$ \\
\hline $\mathrm{CE}_{2}$ enfant $\mathrm{I}^{\circ}$ & 2 & 37 & NN \\
\hline CE2 enfant I I & 6 & 25 & $N+P P$ \\
\hline CE2 enfant I 2 & 3 & 28 & NN \\
\hline $\mathrm{CE}_{2}$ enfant $\mathrm{I}_{3}$ & 3 & 36 & NN \\
\hline $\mathrm{CE}_{2}$ enfant I 4 & 3 & 38 & Mots-valises \\
\hline
\end{tabular}

9 «word-formation paradigms of the language » (nous traduisons). 


\begin{tabular}{|c|c|c|c|}
\hline \multicolumn{1}{|c}{ Sujets } & $\begin{array}{c}\text { Nombre de } \\
\text { stratégies } \\
\text { différentes utilisés }\end{array}$ & \multicolumn{1}{c|}{$\begin{array}{c}\text { Nombre d'éléments } \\
\text { correspondant à la } \\
\text { stratégie majoritaire }\end{array}$} & $\begin{array}{c}\text { Stratégie } \\
\text { majoritaire }\end{array}$ \\
\hline CE2 enfant I 5 & 4 & 2 I & NN \\
\hline CE2 enfant I 6 & 4 & 24 & NN \\
\hline CE2 enfant I7 & 6 & 22 & NN \\
\hline
\end{tabular}

Cette analyse plus qualitative fait également ressortir une variabilité interindividuelle assez importante, et révèle par exemple la faible représentativité du pourcentage de mots-valises relevés chez les $\mathrm{CE}_{2}$, puisqu'un seul des 17 enfants de $\mathrm{CE}_{2}$ interrogés a utilisé une majorité de mots-valises. Les différences observées entre les deux classes n'en sont que plus fortes, mais la variabilité observée et le trop petit échantillon d'enfants de CMr, en même temps que l'absence d'éléments d'évaluation du développement du langage oral de chaque enfant ${ }^{10}$ ne permettent pas de lier ces résultats à un développement langagier plus avancé.

Deux autres éléments ressortent de la séance de questions et d'explications qui a marqué notre dernier passage dans la classe. Tout d'abord, certains au moins avaient fait un usage délibéré du principe d'économie, principe qui a guidé leur choix de suites NN ou de mots-valises. D'autre part, certains enfants se sont montrés sensibles à l'opacité référentielle des mots-valises et ont expliqué ainsi leur choix d'autres stratégies.

\section{Quelques pistes de réflexion issues de la littérature de jeunesse}

Le principe d'économie que certains enfants se sont montrés soucieux de respecter s'illustre également dans de nombreux ouvrages de littérature de jeunesse, où il semble motivé par la recherche de dénominations précéder chaque passation d'un test de langage qui aurait probablement modifié le dispositif expérimental. 
accrocheuses. Ainsi par exemple, dans l'album 365 bisous (de Kimiko, publié en 20 I par l'Ecole des Loisirs), on trouve de nombreuses dénominations comme bisou éléphant, bisou ours blanc, bisou caribou, bisou à ski, bisou en avion, présentées hors syntaxe (il s'agit d'un album à destination de jeunes enfants) et contextualisées uniquement par des illustrations originales.

Une autre hypothèse à examiner est celle d'un éventuel impact de la littérature et des dessins animés traduits sur les productions des enfants. Nous avons demandé aux enfants, après chaque passation, de citer au moins deux des derniers livres qu'ils avaient lus et plus d'un sur deux était un ouvrage traduit. La présence de noms propres comme pokemon (mot-valise en langue anglaise, constitué à partir de pocket et de monster, mais que les enfants francophones ne comprennent certainement pas de cette manière) dans l'environnement linguistique des enfants pourrait par exemple encourager leur créativité et les rendre plus enclins à dépasser les schémas de formation lexicale de la langue, en particulier sur une tâche de dénomination comme celle que nous leur avons proposée. Or c'est bien ce qu’ont fait les enfants que nous avons interrogés, en utilisant en majorité des suites NN et des mots-valises.

En dernière instance, peut-être pouvons-nous faire l'hypothèse de l'existence d'une frontière floue entre suites NN et mots-valises. Taylor (2014) propose que certains termes comme infographique occupent une position intermédiaire, entre composés et mots-valises. Arnaud et Renner (2014) font remarquer qu'il existe des NN français où l'ordre des deux noms ne suit pas l'ordre canonique, puisque le nom qui constitue la tête sémantique se situe régulièrement à droite : ils citent notamment auto pont, radio-crochet, auto radio, bidonville. Ce fonctionnement pourrait s'expliquer par le fait que les noms comme auto sont des troncats ou « bases liées » (Renner 2008 : 150) dont l'usage est relativement fréquent en français. La traduction française du composé anglais Dinosaur-Train par Dinotrain (titre d'une série de dessins animés américains créée par Craig Bartlett) pourrait alors représenter une tendance du français à utiliser dino comme base liée. Le troncat croco présente un fonctionnement similaire (qui s'illustre notamment dans Crocolion, titre d'un ouvrage d'A. Louchard, publié en 2013 aux éditions Thierry Magnier), et dans les deux images où figurait un crocodile, plus de la moitié des enfants ont utilisé croco en combinaison 
avec le second élément de l'image, produisant alors des mots-valises dont le fonctionnement est en réalité extrêmement proche de celui des suites NN. Si l'on accepte que croco fonctionne comme base liée, les mots complexes que sont crocopingouin ou crocomanchot sont en effet construits par juxtaposition plutôt que par « interpénétration » de deux constituants (Bonhomme 2009 : IOI).

\section{Conclusion}

Klein \& Perdue (1997) proposent qu'il existe une «variété de base » des langues, qui correspondrait à une étape d'acquisition au moins pour les locuteurs d'une langue seconde, et au sein de laquelle (nous traduisons): « la formation de nouveaux mots est réduite à la composition nom-nom ${ }^{11}$. Les résultats de notre étude suggèrent que, même dans une langue romane comme le français, les composés ou suites NN (selon l'analyse que l'on en fait) constituent le procédé de création de nouveaux mots le plus souvent choisi par les enfants d'âge scolaire, probablement en partie parce qu'il s'agit du plus facile à produire. Cependant, le nombre non négligeable d'autres stratégies adoptées, et notamment le choix de mots-valises (bien plus présents dans les productions des enfants que nous ne l'aurions imaginé, et qui ont demandé un effort beaucoup plus important aux enfants qui les ont produits) montre que la facilité n'était pas la seule contrainte à l'œuvre.

Dans l'ensemble, les enfants ont tous fait preuve d'une très bonne connaissance des régularités de la langue française, et ce même lorsqu'ils se montraient capables d'aller au-delà : « C'est dans la mesure où nous avons intériorisé les régularités de la langue que nous sommes susceptibles de les transgresser tout en les réaffirmant dans et par la transgression, et c'est

II « Formation of new words is limited to noun-noun compounds », (Klein \& Perdue 1997 : 332). 
aussi dans cette mesure que le sujet récepteur est capable de les identifier. » (Muller $1986: 207$ ).

Enfin, il faut souligner le plaisir qu'ont pris les enfants à participer à cette expérience linguistique, et la curiosité dont ils ont fait preuve, au moment de la séance de questions en classe entière. Il nous a semblé alors que le dispositif expérimental entrait en résonnance avec l'environnement linguistique dans lequel ces enfants évoluent et grandissent : un environnement au sein duquel les néologismes foisonnent, transmis par de nombreux canaux, et où l'image est l'un des principaux vecteurs de nouveauté.

\section{Références}

Arnaud Pierre, 2003, Les composés Timbre-poste, Lyon, Presses Universitaires de Lyon. Arnaud Pierre \& Renner Vincent, 20I4, « English and French [NN]N lexical units : A categorial, morphological and semantic comparison », in Word Structure, $7 / \mathrm{I}, \mathrm{I}-28$.

Bonhomme Marc, 2009, « Mots-valises et remodelage des frontières lexicales », in Cabiers de Praxématique, 53, 99-120.

Bonhomme Marc \& Horak André, 2009, « Stratégies rhétorico-pragmatiques de l'euphémisme dans le discours publicitaire », in Synergies Italie numéro spécial, $5 \mathrm{I}-59$.

Borgwaldt, Susanne R. \& Réka Benczes, 2oII, « Word-formation patterns in a cross-linguistic perspective », in Schönefeld, Doris (Ed.), Converging Evidence: Methodological and theoretical issues for linguistic research, Amsterdam, Benjamins, 22I-246.

Borgwaldt Susanne R., Kulish Tetyana \& Bose Arpita, 2012, « Ukrainian blends : elicitation paradigm and structural analysis », in Renner Vincent, Maniez François \& Arnaud Pierre JL (Eds), Cross-disciplinary perspectives on lexical blending, Berlin/Boston, De Gruyter Mouton, 75-92.

Bücking Sebastian, 2010, « German Nominal Compounds as Underspecified Names for Kinds », in Olsen Susan (Ed.), New Impulses in Word-Formation, Hamburg, Buske, 253-28I.

Clark Eve Vivienne, 198I, « Lexical innovations : how children learn to create new words », in Deutsch Werner (Ed.), The Child's Construction of Language, London, Academic Press, 299-328. 
Clark Eve Vivienne, 1998, « Lexical creativity in French-speaking children », in Cabiers de Psychologie Cognitive, 17, 513-530.

Corbin Danielle, 1987, Morphologie dérivationnelle et structuration du lexique, Lille, Presses universitaires de Lille.

Fradin Bernard, Montermini Fabio \& Plénat Marc, 2009, « Morphologie grammaticale et extragrammaticale », in Fradin Bernard, Kerleroux Françoise \& Plénat Marc (Ed.) Aperçus de morphologie du français, Saint-Denis, Presses Universitaires de Vincennes, 2I-45.

François Frédéric, Francois Denise, Sabeau-Joannet Emile \& Sourdot Marc, 1977, La syntaxe de l'enfant avant 5 ans, Paris, Larousse.

Jackendoff Ray, 2009, « Compounding in the Parallel Architecture and Conceptual Semantics », in Lieber Rochelle \& Štekauer Pavol (Eds), The Oxford Handbook of Compounding, Oxford, Oxford University Press, 436-452.

Kemmer Suzanne, 2003, « Schemas and lexical blends », in Cuyckens Hubert, Berg Thomas, Dirven René \& Panther Klaus-Uwe (Eds), Motivation in Language, Amsterdam, Benjamins, 69-97.

Klein Wolfgang \& Perdue Clive, 1997, « The Basic Variety (or : Couldn't natural languages be much simpler ?) », in Second Language Research, I3, 30I-347.

Lieber Rochelle \& Stekauer Pavol, 2009, The Oxford handbook of compounding, New York, Oxford University Press.

Malmkjaer Kirsten, 2010, The Routledge Linguistics Encyclopedia, third edition, Oxford, Routledge.

Muller Pierre, 1986, « Almuth Grésillon, La règle et le monstre : le mot-valise. Interrogations sur la langue, à partir d'un corpus de Heinrich Heine », in Mots, $13 / \mathrm{I}$, 203-207.

Nicoladis Elena, 2003, « Compounding is not contingent on level-ordering in acquisition », in Cognitive Development, 18, 319-338.

Nicoladis Elena \& Krott Andrea, 2007, « Family size and French-speaking children's segmentation of existing compounds », in Language Learning, 57/2, 20I-228.

Petit Gérard, 2009, « Le mot : morphologie et figement », in Le français moderne, 77/I, 33-45.

Reboul Sandrine, 1996, «L'essor contemporain de l'élément linguistique -tique », in $\operatorname{Lin} x, 8,<$ http://linx.revues.org/II 43>

Renner Vincent, 2006, Les composés coordinatifs en anglais contemporain, Thèse, Université Lumière Lyon 2, <http://tel.archives-ouvertes.fr/docs/oo/56/50/46/ PDF/Renner2006.pdf>

Renner Vincent, 2008, « Quelques remarques sur les quasi-lexèmes de type e- et -holic », in Maniez François \& Dury Pascaline (dirs), Lexicographie et terminologie : histoire de mots, Lyon, Publications du CRTT, I47-156. 
Riegel Martin, 1991, « Ces noms dits composés : arguments et critères », in Studia Romanica Posnaniensia, 16, I48-16I.

Scalise Sergio \& Vogel Irene, 2010, Cross disciplinary issues in Compounding, Amsterdam, Benjamins.

Taylor John R., 20I4, « Prototype effects in grammar », in Dąbrowska Ewa \& Divjak Dagmar (Eds), Handbook of Cognitive Linguistics, Berlin, De Gruyter Mouton. Tomasello Michael \& Brooks Patricia, 1999, « Early syntactic development : A Construction Grammar approach », in Barrett Martyn (Ed.), The development of language. Studies in developmental psychology, New York, Psychology Press, I6I-190. 



\section{Quelle(s) diversité(s) pour la syntaxe?}

\section{Introduction/généralités ${ }^{1}$}

Comme il a été souvent souligné (par exemple, parmi de nombreux auteurs, par Romaine (1984), ou encore, pour le français, par Gadet (1997)), la notion de variation, si on l'entend comme changement de forme sans changement de sens, est délicate à manier en syntaxe, contrairement à ce qui est le cas en phonologie. Ainsi, des « constructions concurrentes, avec un matériau langagier organisé différemment » (Gadet $1997:$ 13), peuvent-elles être sémantiquement équivalentes ? Cette synonymie est admise, au sein de la linguistique variationniste, par Weiner \& Labov (1977: 6), qui, comme le rappelle Romaine (1984:412), définissent les variantes syntaxiques en tant que des « façons alternatives de dire la même chose du point de vue de la vérité ». Néanmoins, la conception majoritaire qui semble avoir émergé est celle d'un assouplissement de la condition d'équivalence sémantique. Telle est notamment la position de Gadet (I997 : II) : « Il faudrait sans doute être moins ambitieux sur les questions de sens, reconnaître les dimensions du flou et du vague, et parler, plutôt que de 'dire la même chose', de 'dire des choses proches à propos d'un même référent' ». Le caractère opératoire de cette notion de proximité sémantique à laquelle on aboutit peut cependant être questionné, d'autant que celle-ci s'accompagne d'une intrication entre sémantique et pragmatique si l'on prend notamment en compte l'intention

I Nous remercions Marie-Hélène Côté pour ses remarques après une communication sur le même thème aux Journées PFC de décembre 2013, Regards croisés sur les corpus oraux - session syntaxe. 
communicative. Sur cette base peuvent être également rapprochés, si l'on choisit deux exemples de Romaine (1984:426), « il fait froid ici » et « pourriez-vous fermer la fenêtre ? ».

Si la diversité, l'hétérogénéité, de la langue a été ramenée à des inventaires de variantes, c'est bien dans le but d'établir des corrélations entre variantes linguistiques et facteurs extralinguistiques, qui passent par les exigences d'une quantification. Typiquement, la variation phonologique est corrélée à des facteurs sociodémographiques qui la conditionnent (tels ceux, habituels à Labov, de sexe, âge, classe sociale, etc.). En revanche, dans le domaine de la syntaxe, ce seraient avant tout des facteurs linguistiques ou situationnels qui joueraient, en raison de la « moindre fréquence [des phénomènes qui] les rend moins saillants pour un investissement social » (Gadet 1997 : 17). Le passage du niveau phonologique au niveau syntaxique prendrait donc également la forme d'un glissement essentiellement du diastratique au diaphasique. Berrendonner et al. (1983) discutent la pertinence de la notion de variante en syntaxe, et le caractère systématique des corrélations entre variables formelles et variables sociales. Gadet (1992) continue la réflexion sur la variation en syntaxe autour du travail de Labov, posant les bases d'une distinction entre l'approche variationniste, dans la suite de la théorie sociolinguistique fondée par Labov, et l'approche variationnelle, qui a plutôt en vue une réflexion théorique sur la diversité langagière inspirée de Coseriu (Gadet 2007).

Par ailleurs, la notion de variation syntaxique est liée de manière privilégiée à une certaine conception, transformationnelle, de la syntaxe, en particulier à la théorie standard de la grammaire générative transformationnelle (Chomsky 1965). En revanche, dans la théorie des principes et des paramètres (Chomsky 198I), les transformations ne sont plus que des déplacements, ce qui ne peut que réduire la nature et le nombre des candidats à la variation syntaxique. Par exemple, si, dans les années soixante, d'une part, « le chat mange la souris » et, d'autre part, « la souris est mangée par le chat », analysé comme étant dérivé de la première structure, sont considérés comme deux variantes, il n'en est plus de même dans les années quatre-vingt et quatre-vingt-dix, où « la souris est mangée par le chat » est dérivé de « est mangée la souris par le chat ». Hors conception transformationnelle, pour Gadet (2010 : I2I), il s’agit de « s’appuyer sur des proximités 
de structures pour explorer les potentialités diversifiées, en tenant compte des fréquences, des contraintes, des affinités et des différences $\gg$.

La proximité sémantique évoquée supra s'accompagne donc d'une proximité de structures. Cet assouplissement à la fois sur un plan sémantique et sur un plan syntaxique pourrait sembler rendre malaisée l'identification de variantes syntaxiques mais, dans les faits, ce sont souvent les mêmes phénomènes qui sont désignés comme relevant de la variation syntaxique:

[Sont] favorables à la variation [syntaxique] les contextes emphatiques, interrogatifs ou les affirmations fortes [...], ou encore la mise en valeur à travers l'ordre des mots [...]. La variation syntaxique serait ainsi sensible à des contextes intrinsèquement interactifs, spécifiques du face-à-face de l'oral. (Gadet $2007: 78$ )

Le besoin de forte saillance de la variable, du point de vue de sa présence quantifiable dans les corpus comme de celui de sa visibilité par rapport au standard, conditionne en partie la recherche en syntaxe. Les recherches récentes en variation syntaxique posent de plus en plus la question du repérage et de la quantification autrement quà partir du français standard (Cheshire 2005).

Les recherches automatiques sont encore embryonnaires pour le français du fait de la carence de grands corpus cumulables et comparables (Cappeau \& Gadet 2007), ce à quoi le projet $\mathrm{PFC,} \mathrm{«} \mathrm{Phonologie} \mathrm{du} \mathrm{français}$ contemporain : usages, variétés, structure $\gg^{2}$, tente de remédier. Proposant actuellement des données orales de trente-six points d'enquêtes en Europe, en Afrique et au Canada, le moteur de recherches en ligne permet quelques requêtes simples sur des mots ou des propriétés. Nous aurons ici l'occasion de discuter la capacité heuristique de requêtes en partie prévues par le paramétrage des outils, outils en outre difficiles à mettre au point pour la syntaxe. Ce ne sera que pour l'illustration de phénomènes connus par ailleurs que nous ferons référence à ce type de requêtes.

Sans procéder à un inventaire des « diversités », nous allons nous pencher plus particulièrement sur certains faits souvent rangés dans la variation syntaxique, les interrogatives totales (directes), la présence ou absence du

2 Corpus PFC, « Phonologie du français contemporain : usages, variétés et structure », <http://www.projet-pfc.net>. 
subjonctif, l'alternance entre conditionnel « classique » et « conditionnel périphrastique » (aller à l'imparfait suivi de l'infinitif), certaines réalisations indéterminées des auxiliaires avoir et être et, enfin, les structures s'organisant autour de la présence $v$ s absence de que, « marque polyvalente de subordination » (Gadet 1989: 163), tout en évoquant quelques questions en arrière-fond.

\section{Le cas de l'interrogation totale (directe)}

L'interrogation totale directe (pour laquelle la réponse attendue est de type oui ou non) est très souvent présentée comme un cas de variation syntaxique, comportant nommément des variantes (le terme est utilisé, si l'on cite quelques travaux récents, par exemple par Coveney (2002) ou encore par Elsig \& Poplack $\left.(2006)^{3}\right)$ bien identifiées, en nombre limité généralement à trois ou quatre structures, caractérisées soit par un ordre particulier (on parle traditionnellement d'inversion), soit par la présence de est-ce que, soit (uniquement) par une intonation montante, soit, enfin, éventuellement, par la présence de $t u$. Ces structures respectives sont représentées ci-après à partir d'exemples de la base PFC :

(i) As-tu une photo ? (Montréal, Côté à paraître)

(2) Est-ce que moi je, est-ce que jai besoin de dire que je suis patron? (Ouagadougou, Prignitz 2007)

(3) Mais elle a pas autre chose à faire que de regarder ça à son âge ? (Darnétal, Hall \& Lyche 2010)

(4) Il y a-tu des places spécifiques? (Peace-River, Walker 2010) ${ }^{4}$

3 L'article d'Elsig \& Poplack (2006) préfigure l'ouvrage d'Elsig (2009): Elsig, Martin, Grammatical Variation Across Space and Time: The French Interrogative System, Amsterdam/Philadelphia : John Benjamins.

4 Néanmoins, notons, sans pouvoir insister davantage, que d'autres possibilités existent, comme le rappelle Gadet (1989 : 137) : Est-ce Pierre qui vient ?; Est-ce que c'est Pierre qui vient ?; C'est Pierre qui vient ?. 
Le traitement de prédilection des interrogatives en termes de variantes ${ }^{5}$ est notamment lié à leur relative fréquence à l'oral (même si elle reste sans commune mesure avec celle de variables phonologiques), nécessaire à des analyses quantitatives, ainsi quà leur répartition marquée d'un point de vue diatopique (pour les interrogatives en $t u$ ) et, surtout, d'un point de vue diaphasique. Comme il est reflété par exemple dans la plupart des manuels de Français Langue Etrangère en France (voir, parmi d'autres, Calas \& RossiGensane (2010)), les formes interrogatives totales sont différenciées quasi exclusivement sur le plan des registres de langue, les structures à inversion étant présentées comme des tournures de la langue soutenue ou soignée, par opposition aux structures introduites par est-ce que ou reposant sur la seule intonation, que l'on fait généralement relever de la langue courante. En cela, l'interrogation totale épouse parfaitement l'une des hypothèses de la sociolinguistique qui est « d'opposer les formes par niveaux de langue [...]. D'où la prédominance d'études mettant en jeu des alternances, surtout entre forme de prestige et forme non standard. » (Gadet 1997 : 8). En outre, dans la théorie des principes et des paramètres, la structure dite traditionnellement à inversion est analysée comme résultant de la structure marquée par la seule intonation, suite à des mouvements (orientés vers la gauche) du verbe. Cette dernière structure et celle introduite par est-ce que se distinguent uniquement par le fait que la position du complémenteur est respectivement vide ou occupée (par est-ce que). On a donc strictement affaire, sur un plan syntaxique, à des variantes délimitées par la grammaire générative (en des termes plus actuels que, par exemple, pour le passif lequel, comme on l'a vu, ne peut plus être mis en relation avec son correspondant à l'actif). Autrement dit, il s'agit de ces cas, appelés de ses vœux par Cheshire (2005), où « generativists [...] help variationists determine on a more systematic basis those syntactic structures that should be considered variants of a single form $\gg$. Néanmoins, si l'on accepte de considérer les différentes structures de l'interrogation totale comme exemplaires de la variation syntaxique, alors il faut convenir que la variation syntaxique ne

$5 \quad$ Elsig \& Poplack (2006) : « Variability in question formation is a well-documented feature of French syntax $\gg$. 
garantit pas une équivalence sémantique, dans la mesure où, comme on va le rappeler, elle ne garantit déjà pas une équivalence pragmatique.

Gadet (1997 : 10) met en garde contre « la manie grammairienne d'assigner un sens à toute variation de forme », que Poplack (2009: 136) interprète encore comme une tentative de la tradition normative d' « éliminer la variabilité » en niant l'interchangeabilité : « c'est-à-dire qu'au lieu d'admettre que les formes expriment toutes la même chose, [les grammaires] prétendent que chacune a une fonction différente ». Les structures interrogatives parfois dotées de nuances sémantiques peu stables fournissent ainsi des exemples de ce que Berrendonner (1988:56), cité par Gadet (1997 : 10), nomme « sémiotisation arbitraire » : «Si ta maman entre dans la cuisine quand tu es assis devant la table et feuillettes un livre, elle te demandera, paisiblement, Que fais-tu ? En revanche, si, juché sur un tabouret, tu es en train de farfouiller dans les pots de confiture au haut de l'armoire, elle t'apostrophera par un brutal Qu'est-ce que tu fais ? ». De même, Flament-Boistrancourt \& Cornette (I999 : I2I) remarquent : « Pour Terry (1970: 99-108), ce que traduit par exemple l'emploi de la structure en est-ce que dans les interrogations totales, c'est l'impatience de connaître la réponse, point de vue que ne partage nullement Coveney (1996:2II) : 'In Yes/No interrogatives, a speaker wishing to elicit the answer from an interlocutor tends to use [SV] [sujet + verbe], whereas a speaker not wishing to elicit an answer tends to use [ESV] [est-ce que + sujet + verbe]' ! ».

Face à cet « éparpillement » sémantique, un traitement pragmatique peut constituer un recours. S'y prêtent particulièrement bien les structures interrogatives, situées sur un versant sémasiologique, qui correspondent de manière privilégiée, sur le versant onomasiologique, à des actes de question exprimant des demandes d'information, ou, selon la formulation de KerbratOrecchioni (I99I : I5), des « demandes d'un dire ». Or, ces structures interrogatives peuvent revêtir d'autres valeurs pragmatiques. Ainsi, est souvent isolée, tout au moins pour le français de France, la structure interrogative signalée par la seule intonation, susceptible de fonctionner comme une demande d'information, mais aussi comme une demande de confirmation (se rapprochant d'une assertion), ou encore comme une requête indirecte (relevant de l'injonction), sans doute du fait que cette structure est « le 
plus faiblement marquée sur le plan formel » (Borillo (1978 : 549), citée par Kerbrat-Orecchioni (1991 : IIO)).

Dans les exemples (I à 4) supra tirés de PFC, la structure interrogative reposant uniquement sur l'intonation est en effet une question rhétorique (ce dont la polarité négative est un indice) équivalant à une assertion, approximativement du type : «A son âge, elle pourrait trouver autre chose à faire ». Est néanmoins également une question rhétorique la structure introduite par est-ce que, qui signifie ici « Je n'ai pas besoin de dire que je suis patron ».

Il semble difficilement défendable de considérer comme variantes des structures interrogatives ne comportant pas une valeur pragmatique identique et c'est bien ce dont prennent acte par exemple Elsig \& Poplack (2006) en excluant d'emblée de leur étude notamment les requêtes indirectes, les questions rhétoriques et les questions échos, sortes de demandes de confirmation. La variation à un niveau strictement syntaxique, même nettement délimitée, apparaît donc comme devant être complétée, en l'occurrence à un niveau pragmatique. Autrement dit, si elle est ici une condition nécessaire, elle n'est en aucun cas suffisante. Par ailleurs, le traitement pragmatique se heurte au phénomène du cumul de significations, où plusieurs sens se superposent et qui peut amener à questionner l'exclusion de certains éléments. Ainsi, par exemple, la structure interrogative « Mais elle a pas autre chose à faire que de regarder ça à son âge ? » (PFC, Darnétal) n’a pas seulement valeur d'assertion, en tant que question rhétorique, mais est aussi interprétable comme une question pour laquelle une réponse - certes, de préférence, d'acquiescement - est attendue.

Il est intéressant de noter, au-delà d'une diversité diatopique, des constatations quantitatives divergentes quant à la représentation des différentes structures interrogatives. Comme il est bien connu, la diversité diatopique se manifeste pour les structures interrogatives par une forme spécifique, celle dotée de $t u$, attestée seulement en français canadien (et plus récente que celle comportant $t i$, rare en France et cantonnée à quelques régions comme la Bretagne ou la Normandie). La structure en $t u$ représente $36 \%$ des interrogatives totales du corpus de français québécois du $\mathrm{XX}^{\mathrm{e}}$ siècle d'Elsig \& Poplack (2006), en revanche seulement 4,5\% du corpus de français québécois de Bigot (2008). La nature de ce dernier corpus, 
constitué d'entrevues journalistiques télévisées, rend sans doute compte de cette différence, dans la mesure où les interrogatives formées avec tu appartiennent au français québécois familier.

Les structures en $t u / t i$ illustrent un cas où variation diatopique et variation diachronique sont liées. Leur évolution différenciée respectivement en français de France et en français du Québec serait notamment due à leur stigmatisation dans la première variété. Poplack (2009: 133) rapporte à ce propos que la « langue idéale ne contiendrait pas la particule interrogative -ti/tu (c'est-tu vrai ?), parce que, tout au moins selon Damourette et Pichon, elle est 'vulgaire' ».

La diversité diatopique se manifeste également, de manière moins évidente, par une certaine répartition des autres structures interrogatives. Cependant, cet ordre de diversité ne saurait masquer les divergences quantitatives entre études.

Pour Blanche-Benveniste (1997:39), « l'interrogation par est-ce que est largement utilisée $\gg$, les exemples qui suivent montrant que cette remarque s'applique tout autant aux interrogatives totales qu'aux interrogatives partielles, mais, pour Flament-Boistrancourt \& Cornette (1999 : I2I), « les études entreprises par les linguistes ont permis de dénoncer nombre d'idées reçues [dont] par exemple l'affirmation selon laquelle est-ce que serait une caractéristique générale de la langue parlée $\gg^{6}$.

De fait, la plupart des études quantitatives sur le français de France s'accordent pour donner une place minoritaire à la structure interrogative totale introduite par est-ce que. Toutefois, la proportion varie de manière non négligeable d'un auteur à l'autre (par exemple $2 \%$ chez De Cat (2007 : I4) et $20 \%$ chez Coveney (2002:246)). En français du Québec, si la part des interrogatives totales de cette sorte est peu importante dans le corpus d'Elsig \& Poplack (2006), où elle est de 6\%, elle est prépondérante dans le corpus de Bigot (2008), où elle représente 78,5\%. Cette énorme disparité

6 Affirmation probablement favorisée par les préconisations des grammaires, telles que celle de Radouant (1922 : 232-33), cité par Poplack (2009 : 133) : «De plus en plus fréquemment, dans la langue parlée, quand la question porte sur le verbe et surtout s'il est à la ì̀re personne du singulier, on emploie la formule invariable est-ce que ». 
peut, sans doute, encore être imputée à la nature formelle de ces dernières données, car les interrogatives totales introduites par est-ce que sont considérées comme relevant d'une langue soutenue en français québécois ${ }^{7}$.

Les interrogatives totales reposant sur la seule intonation sont extrêmement majoritaires en français de France, même si les pourcentages varient selon les études ( $80 \%$ pour Coveney (2002 : 108), $98 \%$ pour De Cat (2007 : I4)). En français québécois, où il peut être prêté à ces structures un certain caractère formel (toutefois moindre que pour celles en est-ce que), leurs proportions sont plus faibles tout en différant parfois sensiblement : $61 \%$ pour De Cat (2007 : 14), 35\% pour Elsig \& Poplack (2006) et 7\% pour Bigot (2008:232), malgré ce que pouvait laisser attendre la nature du corpus.

Pour le français de France, Coveney (2002: 246) note une complète absence d'interrogatives totales à inversion, de même que De Cat (2007: I4), conformément à ce que remarque Flament-Boistrancourt (200I) : « Toutes les études sont unanimes à ce sujet : la structure à inversion est rare à l'oral ». La constatation est, bien sûr, à relativiser sur l'axe diatopique. Les interrogatives totales à inversion représentent 26\% dans le corpus de français québécois d'Elsig \& Poplack (2006), 21\% dans celui de De Cat (2007 : I4) et 9,5\% dans celui de Bigot (2008 : 232). Pour Elsig \& Poplack (2006), l'inversion n'affecte que la deuxième personne alors que, pour De Cat (2007: 13), elle concerne également la troisième personne.

Enfin, la «prédilection du francophone pour l'interrogation totale » (Flament-Boistrancourt 200I) est souvent soulignée, et ce de manière convergente, pour le français de France. Ainsi, Flament-Boistrancourt \& Cornette (1999 : 137-139) notent 60,50\% d'interrogatives totales contre $39,50 \%$ d'interrogatives partielles. Ils citent également le ratio de 69,09\% de totales contre $30,90 \%$ de partielles pour Terry (1970: 83 ) et de 66,92\% de totales contre $24,61 \%$ de partielles pour Gadet (1989: I 43). Coveney (2002 : I18) observe de son côté une fois et demie plus d'interrogatives

7 Elsig \& Poplack (2006) expliquent ce trait diaphasique par la rareté de la structure en est-ce que dans le français de France du XVII ${ }^{e}$ siècle, dont le système interrogatif du français québécois paraît directement provenir. Il semblerait en effet, selon Rouquier (2002), que est-ce que ne soit apparu que tardivement, au XVI ${ }^{\mathrm{e}}$ siècle, dans les interrogatives totales. 
totales que d'interrogatives partielles. En revanche, cette tendance demande à être vérifiée pour le français québécois, du fait de résultats à première vue contradictoires : si Barbarie (1982), cité par Bigot (2008 : 229), relève, sur 776 structures interrogatives directes, 436 totales et 340 partielles et si Bigot (2008 : 230), sur 240 structures, dégage 130 totales et i Io partielles, Flament-Boistrancourt (2004) rapporte l'étude de Karcher (1999), qui montre une prépondérance des partielles ( $50 \%$ contre $40 \%$ de totales) dans son corpus de Montréal ${ }^{8}$.

Pour démêler ces divergences portant sur un phénomène autant culturel que linguistique, il est sans nul doute nécessaire de disposer de davantage de corpus, mieux échantillonnés, plus comparables, avec des situations diversifiées de manière plus systématique, qui donneraient les moyens d'une approche privilégiant, comme on l'a vu, le diaphasique.

\section{Modes et temps : les cas du subjonctif, du « conditionnel périphrastique » et des auxiliaires avoir et être}

Les études de la variation des modes et des temps verbaux, tout comme celles de la variation entre les auxiliaires avoir et être, sont classiques en syntaxe. La facilité de comparer chaque forme avec la variante standard, étant donné l'investissement normatif de la zone verbale, pourrait en être la raison, tout comme la possibilité de réduire la variation à un cadre binaire qui permet une quantification aisée. De fait, les temps verbaux et leurs environnements sont relativement faciles à « traquer » par les outils automatiques, bien que ceux-ci restent dépendants des concepteurs et, surtout, des transcripteurs

8 Cette étude examinait la formulation des demandes d'information à la banque de prêt d'une bibliothèque universitaire parisienne et d'une bibliothèque universitaire de Montréal. La proportion d'interrogatives totales (70\% contre $20 \%$ de partielles) restait plus élevée pour le français de France. 
des corpus, et que les études soient limitées (pour le subjonctif $v$ s indicatif, par exemple) aux formes contrastées.

Une « tendance » à l'amenuisement du subjonctif est observée par de nombreux auteurs dans plusieurs variétés de français. L'alternance du subjonctif et de l'indicatif dans des subordonnées est en réalité une donnée du français attestée depuis le XVIe siècle, toujours accompagnée des injonctions normatives visant à stabiliser la distribution des usages (Fournier 1998: 331-373). Le subjonctif en subordonnée exclut l'assertion du locuteur; il focalise l'attention sur le procès exprimé sans qu'il soit tenu compte de sa réalité. La pertinence de cette valeur modale, cependant, ne va pas toujours de soi puisque le subjonctif en tant que forme marquée n'existe pas pour de nombreux verbes. Par ailleurs, dans les usages, des études ont montré (surtout pour le français acadien (Chauveau (1998), Neumann-Holzschuh (2004), parmi d'autres), selon les contextes, soit une fluctuation imprévisible entre subjonctif et indicatif (après falloir, vouloir, pour que, avant que ...), soit une absence du subjonctif (après jusquả ce que, il arrive que ...). Chauveau (1998) rapproche ces faits de la tendance identique attestée de longue date en Bretagne, tendance correspondant elle-même au fonctionnement du breton où l'indicatif présent et futur et le conditionnel (utilisé aussi pour les constructions hypothétiques) sont employés là où le français (standard) recourt au subjonctif. NeumannHolzschuh (2004), dans son étude formelle et fonctionnelle du subjonctif en français acadien, et plus généralement en français d'Amérique du Nord, montre aussi un étiolement du subjonctif, et fait remonter le processus aux XVI et XVII e siècles, donc aux débuts du français sur le continent. Elle met en évidence une apparition concurrente des formes de l'indicatif présent, futur et imparfait, du conditionnel et du subjonctif présent, avec la perte de la valeur modale spécifique du subjonctif, surtout en Louisiane et à Terre-Neuve et, dans une moindre mesure, au Nouveau-Brunswick et en Nouvelle-Ecosse. En France, au Québec et en Afrique, les alternances du subjonctif et de l'indicatif et la diminution du subjonctif sont attestées aussi. Des données récentes de français parlé en entretiens et conversations comportent quelques absences de subjonctif après $\dot{a}$ moins que, falloir que, avoir peur que, jusqu’à ce que, malgré que ..., mais ces faits restent marginaux : 
(5) À moins que ... quelqu'un il ... il me voyait là, but je pense pas (Peace-River, Walker 2010)

(6) Faudra que tu te fais ton idée (Ville Platte, Lyche, Klingler \& LaFleur 20ıо)

(7) Il avait peur que j'aurais perdu connaissance (Ilet à Cordes, Bordal \& Ledegen 2010)

(8) Mais mi di : il faut que vous me dites (Ilet à Cordes, Bordal \& Ledegen 2010)

(9) Ainsi de suite jusqu'à ce qu'on va l'employer officiellement (Ouagadougou, Prignitz 2007)

(Io) Les étudiants malgré qu'ils ont des diplômes, mais ils ont des difficultés pour acquérir ces bourses (Dakar, Guèye 2008)

Une comparaison après falloir que dans trente-six points d'enquêtes PFC en Europe, Afrique et Canada ${ }^{9}$ montre seulement trois indicatifs, tous à Ilet à Cordes (Réunion), sur 230 occurrences. Les résultats de diverses études dépendent souvent des corpus (Gandon 1994, Boutin 2007).

Parallèlement à de telles «tendances » répandues dans toute la diatopie du français, des phénomènes concernant des zones géographiques plus ou moins étendues ont aussi été repérés. Ainsi, la spécialisation de l'auxiliaire aller à l'imparfait suivi du verbe à l'infinitif (qui est utilisé en construction libre dans tous les français) dans l'apodose d'une construction hypothétique fictionnelle semble spécifique à la Côte d'Ivoire, au Mali et au Burkina Faso (Boutin \& Kouamé 20II, Skattum 20II) :

(II) Si on avait pu déplacer tous les Gurunsi là, ça allait faire de la place (Ouagadougou, Prignitz 2007)

(I2) Si il était allé à l'école, il allait la lire lui-même (Bamako, Skattum 20II)

(13) Bon, ils nous avaient dit au départ que, bon, si on avait la chance, que ils allaient nous garder (Abidjan, Boutin 2006)

Une comparaison à l'aide du logiciel $\mathrm{TXM}^{10}$ entre le conditionnel classique ou synthétique et ce conditionnel périphrastique montre une équivalence du nombre d'emplois dans les trois enquêtes : après les 17 protases en $s i$

9 En l'état actuel de la base PFC (<http://www.projet-pfc.net/carte-interactive.html>), ces données quantifiées ne comprennent pas la Louisiane ni les Antilles, mais seulement l'Alberta (Peace River) et Québec-ville pour l'Amérique. 
suivies de l'imparfait, 8 apodoses avec un conditionnel classique apparaissent, pour 9 apodoses avec un conditionnel périphrastique.

Un autre niveau linguistique est étroitement uni à la syntaxe : le niveau phonique, dans lequel, par ailleurs, on trouve la plus grande amplitude de variation diatopique. Des faits d'ordre phonologique peuvent être responsables d'une extension de la variation, ou susceptibles de favoriser un changement morphosyntaxique. En Côte d'Ivoire et au Burkina Faso, les auxiliaires ai, as, a, es, est, aie, aies, ait, et parfois êtes, avez, sont réalisés par les voyelles $[\varepsilon / a]$, sans qu'une valeur phonétique précise soit combinée de façon stable à un même verbe. Le symbole $Æ$ indique dans les exemples suivants des voyelles mi-ouvertes à ouvertes, proches de $[\varepsilon / \mathrm{a}]$, qui semblent s'opposer à la voyelle attendue :

(I4) Donc c'est par rapport à ça qu'on vous $Æ$ on vous $Æ$ vus dans les problèmes (Ouagadougou, Prignitz 2007)

(is) On peut pas toujours rester toujours comme on le dit avec les colonisateurs c'est tel pays qui nous $Æ$ colonisés il faut rester avec lui non (Abidjan, Boutin 2006)

(I6) Je l' $Æ$ pas vu il $Æ$ fui avec mon argent (Abidjan, Boutin 20ıb)

Est-on en présence d'un changement grammatical en cours, un morphème Æ devenant simple marqueur de l'aspect accompli ? Ces faits sont-ils suffisants pour qu'on en induise qu'il y a changement dans le système ?

Pour ces diverses valeurs phonétiques proches de $[\varepsilon / \mathrm{a}]$, on peut émettre l'hypothèse d'une forme syncrétique au niveau phonologique, mais on ne peut supposer pour autant qu'il s'agisse d'un changement morphosyntaxique. Cette forme syncrétique est le résultat d'une perte d'opposition phonologique [a $\sim \varepsilon]$ pour certains morphèmes ( $a i, a$, as, es, est ...) et d'une érosion consonantique pour d'autres (êtes, avez). Néanmoins, pour que l'on puisse poser l'existence d'un seul morphème $[a / \varepsilon]$ ou de plusieurs (plutôt $[a]$ et plutôt $[\varepsilon]$ ), une grande régularité doit être observée. Or, ce phénomène est très variable; la fréquence de $Æ$ diffère selon les situations de parole, estimée à moins de dix fois en cinq heures de parole dans les entretiens de PFC (0,002\% des occurrences d'être et avoir au présent) et vingt-cinq fois en dix minutes d'enregistrement écologique dans une gare routière d'Abidjan (23\% des occurrences d'être et avoir au présent) (Boutin 2orrb). Il semble que cette imbrication de faits 
d'ordres phonique et syntaxique soit elle-même dépendante d'autres facteurs (acquisition, situation de communication, vitesse de débit).

\section{Le cas de que}

Le cas de que permet d'aborder d'autres conditions d'existence de la diversité syntaxique, notamment la dispersion concomitante d'emplois plus ou moins grammaticalisés d'une même forme, autrement dit l'interdépendance entre la syntaxe et le discours. Le cas de que permet aussi de (re)poser de manière particulièrement aiguë le problème de l'identification et de la délimitation de formes porteuses de constructions concurrentes (est-ce à dire : de variantes ?). La diversité des emplois de que en français est en effet telle qu'elle va des subordonnées où que est subordonnant et première marque d'une réelle dépendance syntaxique, aux énoncés paratactiques où que a un rôle de particule énonciative, en passant par que subordonnant universel ou lien discursif vague :

(17) on avait juste eu l'électricité que moi j'avais I6 ans (Louisiane, Boutin \& Gadet 2012)

Le sous-corpus PFC-Afrique de l'Ouest (Sénégal, Côte d'Ivoire, Mali et Burkina Faso) présente cent trente relatives en que, dont trois avec un que pouvant encore être considéré relatif, mais un relatif non marqué en fonction, éventuellement suivi d'un pronom résomptif. Ces exemples, que l'on fait respectivement relever desdites relatives défectives et desdites relatives décumulées, sont semblables à ce que l'on rencontre dans tous les espaces francophones :

(I8) Ensemble, nous devons lutter aussi contre plein de choses. Il y a plein de choses que nous devons lutter, la corruption, euh (Abidjan, Boutin 2006)

(19) Les vrais, les vrais mots que on a besoin, ils disent pas ça en bambara (Bamako, Skattum 2008)

(20) Vraiment, il y avait des choses que on se plaint pas s /-s /-sur ça (Bamako, Skattum 2008) 
Ces deux sortes de relatives sont stigmatisées comme « populaires ». En très petit nombre dans le corpus PFC-Afrique de l'Ouest, elles sont pourtant fréquemment mises en évidence dans la plupart des études sur le français en Afrique, ce qui manifeste une intrication de facteurs diastratiques et diatopiques, non seulement au niveau des phénomènes observés, mais aussi au niveau de la démarche des chercheurs. S’il existe une préférence, dans les études sur le français en Afrique, pour les «français populaires » ou les formes dites « populaires », il se peut que les méthodes soient encore tributaires de l'idéologie du standard corrélé à une position sociale haute.

Par ailleurs, que est absent dans ce qui est traditionnellement analysé comme des complétives :

(2I) Et, quand je lui ai expliqué c'est le lavement, elle croit pas (Abidjan, Boutin 2007)

(22) Je savais, quand elle sévissait, c'était vraiment qu'elle avait ses raisons (Meurtheet-Moselle, Pagliano \& Le Gac 20ıо)

(23) Parce que je trouve où il y a des fermes il n'y a pas autant de changement de personnes (Ontario, Poiré 2010)

Autour de cette présence $v$ absence de que, quelles formes comparer et constituer en paradigme(s) ? Et qu'est-ce qui alterne ? Est-ce des énoncés, des structures, des éléments, tel ce que présent ou absent ? Selon Gadet (1997: II-I2), « on peut rapprocher relatives et détachements, car en français ordinaire que est souvent supprimé, même ailleurs qu'en relative $[\ldots]$. [...] une relative à laquelle on retire que est un détachement, et un détachement auquel on ajoute que donne une relative. Les locuteurs sont donc familiers de l'interchangeabilité entre structures avec et sans que ». En ce qui concerne les apparentes complétives, l'absence de que est à relier au fait que expliquer, savoir et trouver (2 I à 23) appartiennent aux introducteurs de discours rapporté (voir par exemple Rosier 2008 : 56-57). En particulier, un verbe tel que trouver, très fréquemment attesté, à l'oral, à la première personne (comme l'illustre l'exemple (23)), a majoritairement un emploi dit de « rection faible, à valeur épistémique » (Blanche-Benveniste $\&$ Willems 2007 : 217). Pour les verbes de cette sorte, Blanche-Benveniste \& Willems (2007:226) notent « en France [...] un affaiblissement du que, qui tendrait à s'agglutiner au verbe faible, je crois-que, je pense-que », tout en 
précisant que « l'absence du que est signalée en français québécois (Sankoff 1974) et parfois en français parisien (Andersen 1997) ».

En relation à l'absence de que, l'exemple (17) on avait juste eu l'électricité que moi javais 16 ans peut être mis en correspondance avec un cas (patent) de parataxe, on avait juste eu lélectricité, moi javais 16 ans, décrit par Choi-Jonin \& Delais-Roussarie (2006) comme une « association de propositions sans marque segmentale $\gg$. Une telle suite se paraphraserait en écrit standard à l'aide d'une subordonnée, notamment introduite par quand. Choi-Jonin \& Delais-Roussarie (2006:94) notent cependant, pour ladite association de propositions sans marque segmentale, des contraintes syntactico-sémantiques ${ }^{11}$ « qui ne s'observent pas dans les propositions reliées par [un] subordonnant » (voir aussi Blanche-Benveniste et al. 1990 : 139-140). Autrement dit, les différentes formes, avec que, sans que, avec un subordonnant tel que quand, ne sont pas strictement équivalentes sur un plan syntaxique et sur un plan sémantique. En outre, que, « marque polyvalente de subordination » (Gadet 1989: 163), est notamment doté d'un plus grand potentiel syntaxique et sémantique (qui se manifeste par exemple dans le fait que certaines relatives décumulées peuvent également être interprétées comme des circonstancielles, ou encore dans le fait que les paraphrases peuvent s'appuyer sur plusieurs subordonnants : on avait juste eu l'électricité alors que moi j'avais ib ans).

A la faveur de l'exemple on avait juste eu l'électricité que moi javais ib ans, nous souhaiterions également évoquer l'apport possible des cadres théoriques de micro et macrosyntaxe dans le domaine de la diversité syntaxique. La macrosyntaxe, définie au début des années quatre-vingt-dix, d'une part à Aix par Blanche-Benveniste et al. (1990), d'autre part à Fribourg par Berrendonner (1990), comme une description de l'au-delà de la rection, par opposition à la (micro)syntaxe, syntaxe de rection, prend en effet tout particulièrement en compte des faits de parataxe. Nous nous cantonnerons

II La première proposition « ne peut pas être négative ». Du point de vue des temps verbaux, « seuls les ordres accompli-inaccompli et inaccompli-inaccompli sont acceptés ». Si la deuxième proposition «n'exprime pas une indication temporelle, la première doit comporter un prédicat du type ponctuel ». (Choi-Jonin \& DelaisRoussarie 2006:94) 
ici à une brève discussion de cet exemple au sein du cadre fribourgeois. Rappelons que ce cadre théorique est doté d'unités maximales de microsyntaxe d'une seule sorte, les clauses, décrites comme des « îlots de connexité rectionnelle », où « chaque élément (morphème, syntagme) [...] est en relation de dépendance ou d'interdépendance avec au moins un autre élément, si bien que 'le tout se tient' » (Berrendonner 2004). Ce cadre possède également des unités maximales de macrosyntaxe, les périodes, que l'on définira d'une manière simplifiée comme des suites de clauses (mais qui consistent parfois en une seule clause) évoquant un programme pragmatique ou praxéologique complet et se terminant par une prosodie conclusive. Ainsi, pour Béguelin (2000), si l'exemple on avait juste eu l'électricité que moi javais 16 ans est certainement constitué d'une clause coextensive avec une période, son correspondant sans que, on avait juste eu l'électricité, moi javais 16 ans, comporte deux clauses appartenant à une même période. Toutefois, par la suite, Berrendonner (2004) affiche une position différente : notant que, dans le dernier cas, les deux segments ne sont pas « 'juxtaposés' n'importe comment, mais liés par des marques prosodiques spécifiques (pas de pause entre eux + intonation dominante sur le second + pause après) », il pose alors un subordonnant « de nature prosodique », qu'il assimile à un subordonnant « segmental ». Les deux segments sont ramenés à une seule clause, « dont la connexité rectionnelle est [...] signalée par un marqueur de subordination de nature prosodique $\gg$. Selon Berrendonner (2004), ce qui oppose l'exemple supra et son correspondant sans que, ce n'est plus le nombre de clauses (respectivement une $v s$ deux), mais la différence de nature du subordonnant (respectivement segmentale $v$ s prosodique). Quoi qu'il en soit, et au-delà de ses évolutions internes, la macrosyntaxe, à côté par exemple de la grammaire générative, fournit des bases intéressantes sur lesquelles opposer des formes porteuses de constructions concurrentes.

D’autres débats proches impliquant que, autour du discours rapporté, mêlent aussi des questions de syntaxe et des questions de discours, et concernent également tout l'empan des espaces francophones.

Le discours rapporté permet particulièrement de montrer l'imbrication entre la syntaxe et le discours lors d'une pratique sociale (recourir au déjà dit) partagée par toutes les cultures. Boutin (20r ra) montre, sur le sous-corpus PFC-Afrique de l'Ouest, un large éventail de constructions qu'on ne peut 
ramener aux catégories de discours rapportés directs et indirects. Hormis les cas (majoritaires) où que introduit un discours rapporté indirect prototypique comme une première marque de dépendance syntaxique $e^{12}$, que peut être très loin du verbe dans un discours direct ou indirect, ou, comme on l'a remarqué supra (2I), omis pour un discours rapporté indirect. On peut noter, pour les verba dicendi (du type dire, répondre, etc. (Rosier 2008 : 56)), ce qui peut être analysé comme un affaiblissement du rôle syntaxique de que au profit d'un rôle discursif. Que a alors un rôle d'organisation du discours, proche de celui de particules énonciatives telles que non, bon, ben, comme on le voit dans les énoncés suivants (24 à 29). Le balisage assuré par non, ben, etc. (《ligateurs énonciatifs » (Morel \& Danon-Boileau 1998)) semble également pris en charge par que, puisque les débuts de paroles rapportées sont marqués par une ou deux particules énonciatives, ou par que éventuellement précédé ou suivi d'une particule énonciative (24 et 25 ). Des phénomènes de ce type laissent penser que la contiguiité de ces éléments lors du discours pourrait être une motivation à la réanalyse de que comme balise introductrice et décrocheur énonciatif (Rosier 2008).

(24) vers la fin il me dit que non mon papier il n'a pas pu prendre que c'est trop compliqué que lui il pensait que c'était un autre travail et moi je lui ai d-donné un travail que son ami ne peut pas faire que c'est trop pour son ami. (Abidjan, Boutin 201ra)

(25) le gars il dit non que non que le que l'onc- que Monsieur euh Kaboré qu' il est sorti. (Ouagadougou, Prignitz 2007)

(26) je demandais souvent aux enfants si c'était bon c'est quelle école qui était habillée comme ça. (Ouagadougou, Prignitz 2007)

(27) il dit bon euh, l'embellie est finie pour le moment. (Liège, Hambye \& Simon 2006)

(28) il me dit qu'est-ce qui se passe je dis non je viens de la Justice ils m’ont donné un délai qui ne m’arrange pas tout ça (Abidjan, Boutin 2orıa)

(29) Fabienne me dit ben euh, c'est bien la preuve de, de (Liège, Hambye \& Simon 2006)

I2 Les autres marques de dépendance sont les suivantes : la phrase qui suit perd son autonomie énonciative, les temps et modes verbaux sont modifiés, les personnes des pronoms et les flexions verbales subissent un transfert, les repérages spatiaux et temporels sont ceux de la narration. 
Si que garde son rôle syntaxique de subordonnant dans la majorité des cas, il assume aussi, tout comme les particules énonciatives, un rôle discursif de balise de début de discours, qu'il s'agisse de la voix du locuteur ou de la voix rapportée. Ont de fait été mentionnés, en français du Cameroun, des énoncés introduits par que, et ce en l'absence de tout verbe rapportant (Boutin 201ra ; voir aussi Queffelec 2006):

(30) Que je n'ai rien à faire avec l'argent pour acheter ça ?

(31) Que quand le kilométrage dépassait comme ça, vous-même vous n'aviez pas peur de couler votre moteur ? (Onguene Essono, Boutin 2orıa)

L'entrée de que dans l'inventaire des balises introductrices de discours n'a pas fait l'objet d'études hors de l'Afrique. Des divergences d'intérêts et de méthodes pourraient expliquer cette disparité, alors que le phénomène ne se limite probablement pas à ce continent.

\section{Conclusion}

Après avoir attiré l'attention sur l'insuffisance des notions de variables et de variantes comme outils de travail sur la diversité des formes et la multiplicité des façons de dire dans une langue, nous avons revisité quelques phénomènes de prédilection dans le domaine de la variation, en les observant sur l'ensemble des espaces de la francophonie.

Le traitement des diversités syntaxiques se heurte à une double intrication : celle de la syntaxe avec les autres niveaux linguistiques (phonique, prosodique, sémantique, pragmatique, discursif) et celle des facteurs extralinguistiques entre eux, notamment diatopiques et diaphasiques. A cela s'ajoute une interdépendance d'ordre méthodologique et/ou culturel entre les faits recueillis et les démarches des chercheurs.

Jusqu’à présent, les études variationnistes se sont focalisées sur les facteurs diaphasiques sans en avoir toujours réellement les moyens en termes de ressources et d'outils d'analyse, et celles qui ont appréhendé la diversité diatopique, plus clairement segmentée, se sont difficilement libérées des 
attributions diastratiques qui accompagnent cette dernière de façon tenace (Gadet 2007). Néanmoins, les français « non hexagonaux » produisent un effet de loupe sur des phénomènes généraux, alors que, longtemps, ils n'ont été examinés que d'un point de vue didactique, par rapport au français standard.

Enfin, il est également intéressant, pour cerner les diversités en syntaxe, de faire appel à d'autres cadres que la grammaire générative, qui a prévalu dans les approches de la variation. Ainsi, les cadres de micro et macrosyntaxe fournissent un éclairage complémentaire sur les formes porteuses de constructions concurrentes.

\section{Références}

Béguelin Marie José (Ed.), 2000, De la phrase aux énoncés : grammaire scolaire et descriptions linguistiques, Bruxelles, De Boeck/Duculot.

Berrendonner Alain, 1990, « Pour une macro-syntaxe », in Travaux de Linguistique, 2I, 25-36.

Berrendonner Alain, 2004, « Grammaire de l'écrit vs grammaire de l'oral : le jeu des composantes micro et macrosyntaxiques », in Rabatel Alain (Ed.), Interactions orales en contexte didactique: mieux (se) comprendrepour mieux (se) parler et pour mieux (s')apprendre, Lyon, Presses Universitaires de Lyon, 249-264.

Berrendonner Alain, Le Guern Michel \& Puech Gilbert, 1983, Principes de grammaire polylectale, Lyon, Presses Universitaires de Lyon.

Bigot Davy, 2008, «Le point » sur la norme grammaticale du français québécois oral, Thèse de doctorat, Université du Québec à Montréal.

Blanche-Benveniste Claire, 1997, Approches de la langue parlée en français, Paris, Ophrys. Blanche-Benveniste Claire \& Willems Dominique, 2007, « Un nouveau regard sur les verbes faibles », in Bulletin de la Société de Linguistique de Paris, 102/1, 217-254.

Blanche-Benveniste Claire, Bilger Mireille, Rouget Christine \& van den Eynde Karel, 1990, Le français parlé, études grammaticales, Paris, CNRS Editions.

Bordal Guri \& Ledegen Gudrun, 2010, « Conversation à Ilet à Cordes (Ile de La Réunion) : arrivée du téléphone et de la route », in Detey Sylvain, Durand Jacques, Laks Bernard \& Lyche Chantal (Eds), Les variétés du français parlé dans l'espace francophone. Ressources pour l'enseignement, Paris, Ophrys, 283-294. 
Boutin Akissi Béatrice, 2006, Corpus PFC-CIA <http://www.projet-pfc.net/ base-de-donnees/enquetes>

Boutin Akissi Béatrice, 2007, «De et que subordonnants, et variation en français », in $\operatorname{LINX}, 57,57-68$.

Boutin Akissi Béatrice, 201ra, « Traces de l'énonciateur dans le discours rapporté : les particules énonciatives et que indicateurs de quelle parole ? », in Jaubert Anna, Lopez Munoz Juan-Manuel, Marnette Sophie, Rosier Laurence \& Stolz Claire (Eds), Citations I. Citer à travers les formes. Intersémiotique de la citation, Louvain-la-Neuve, Academia Bruylant, 39-55.

Boutin Akissi Béatrice, 20Irb, Corpus CIEL-F-CI, <http://www.ciel-f.org>

Boutin Akissi Béatrice \& Gadet Françoise, 20I2, « Comment ce que montrent les français d'Afrique s'inscrit/ne s'inscrit pas dans les dynamiques des français dans une perspective de francophonie », in Le français en Afrique, 27, Nice, ILF \& CNRS, $19-34$.

Boutin Akissi Béatrice \& Kouamé Kouakou, 20II, « Analyse énonciative comparée des systèmes hypothétiques en $s i$ en français de Côte d'Ivoire et en $s \varepsilon$ en baoulé dans un discours fictionnel », in Autour du verbe, Le français en Afrique, 26, Nice, ILF \& CNRS, 7I-84.

Calas Frédéric \& Rossi-Gensane Nathalie, 2010, « Les discours grammaticaux : changements ou déguisements ? », in Galatanu Olga, Pierrard Michel, Van Raemdonck Dan, Damar Marie-Eve, Kemps Nancy, Schoonheere Ellen (Eds), Enseigner les structures langagières en FLE, Berne, Peter Lang (série GRAMM$\mathrm{R})$, IOI-II2.

Cappeau Paul \& Gadet Françoise, 2007, « L’exploitation sociolinguistique des grands corpus. Maître-mot et pierre philosophale », in Revue Française de Linguistique Appliquée, I2, 99-IIO.

Chauveau Jean-Pierre, 1998, « La disparition du subjonctifà Terre-Neuve, Saint-Pierreet-Miquelon et en Bretagne : propagation ou récurrence ? », in Brasseur Patrice (Ed.), Français d'Amérique : variation, créolisation, normalisation, Université d'Avignon, Avignon, CECAV, ios-119.

Cheshire Jenny, 2005, « Syntactic variation and spoken language », in Cornips Leonie \& Corrigan Karen (Eds), Syntax and Variation: Reconciling the Biological and the Social, Amsterdam, John Benjamins, 81-106.

Choi-Jonin Injoo \& Delais-Roussarie Elisabeth, 2006, « L'association des propositions sans marque segmentale », in Bril Isabelle \& Rebuschi Georges (Eds), Coordination et subordination: typologie et modélisation, Faits de Langues, 28, 83-94.

Chomsky Noam, 1965, Aspects of the Theory of Syntax, Cambridge (MA), MIT Press (trad. fr. Aspects de la théorie syntaxique, Paris, Seuil, 1971). 
Chomsky Noam, 1981, Lectures on Government and Binding: The Pisa Lectures, Holland, Foris Publications (trad. fr. Théorie du gouvernement et du liage, Paris, Seuil, 1991). Côté Marie-Hélène, à paraître, Corpus PFC-CMA, <http://www.projet-pfc.net/ base-de-donnees/enquetes>

Coveney Aidan, 2002, Variability in Spoken French : A Sociolinguistic Study of Interrogation and Negation, Bristol, Elm Bank.

De Cat Cécile, 2007, French Dislocation: Interpretation, Syntax, Acquisition, Oxford, Oxford University Press.

Detey Sylvain, Durand Jacques, Laks Bernard \& Lyche Chantal (Eds), 2010, Les variétés du français parlé dans l'espace francophone. Ressources pour l'enseignement, Paris, Ophrys.

Elsig Martin \& Poplack Shana, 2006, « Transplanted dialects and language change : question formation in Quebec », in University of Pennsylvania Working Papers in Linguistics, 12 /2, 77-90.

Flament-Boistrancourt Danièle, 200I, « Pragmatique et approche communicative : la contribution du corpus Lancom », in Le français dans le monde. Recherches et Applications. Théories linguistiques et enseignement du français aux non-francophones, numéro spécial, Paris, Clé International, I43-170.

Flament-Boistrancourt Danièle, 2004, « Quelle contribution un corpus natifs/nonnatifs est-il susceptible d'apporter à la linguistique et à l'enseignement du français L2 : le cas de LANCOM ? », Communication à la Journée d'étude « Fransk år 200I $\gg$ (Université de Roskilde, 27 avril 200I), Pré-textes franco-danois, IV, Publication de l'Université de Roskilde (Danemark), 45-69.

Flament-Boistrancourt Danièle \& Cornette Greet, 1999, « Bon français ou vrai français ? Une étude de l'acte de question menée à partir d’un extrait du corpus $L A N C O M$ : les scènes dites du baby-sitting », in Sémantique, interprétation et effets syntaxiques, Travaux de linguistique, 38, I19-153.

Fournier Nathalie, 1998, Grammaire du français classique, Paris, Belin Sup-Lettres. Gadet Françoise, 1989, Le français ordinaire, Paris, Armand Colin.

Gadet Françoise (Ed.), 1992, Hétérogénéité et variation : Labov, un bilan, in Langages, 108.

Gadet Françoise, 1997, La variation, plus qu'une écume, in Langue française, 115, 5-18. Gadet Françoise, 2007, La variation sociale en français, Paris, Ophrys.

Gadet Françoise, 2010, « Sociolinguiste dans une grammaire : la variation pour une grammaire du français », in Iliescu Maria, Siller-Runggaldier Heidi, Danler Paul (Eds), Actes du XXVìme Congrès International de Linguistique et Philologie Romanes d'Innsbrück, Berlin, De Gruyter, I17-I25.

Gandon Francis-Marie, 1994, «Appropriation et syntaxe du français écrit dans la presse de Ouagadougou (Burkina Faso) : prépositions, rections, pronoms », in Le français en Afrique noire, faits d'appropriation, Langue française, 104, 20-36. 
Guèye Gabriel Marie, 2008, Corpus PFC-SNA, <http://www.projet-pfc.net/ base-de-donnees/enquetes>

Hall Damien \& Lyche Chantal, 2010, « Conversation à Darnétal (Seine-Maritime) : la télévision dans les loisirs », in Detey Sylvain, Durand Jacques, Laks Bernard \& Lyche Chantal (Eds), Les variétés du français parlé dans l'espace francophone. Ressources pour l'enseignement, Paris, Ophrys, 35-46.

Hambye Philippe \& Simon Anne-Catherine, 2006, PFC-BLA, <http://www.projetpfc.net/base-de-donnees/enquetes>

Kerbrat-Orecchioni Catherine (Ed.), 1991, La question, Lyon, Presses Universitaires de Lyon.

Lyche Chantal, Klingler Thomas A. \& LaFleur Amanda, 20ı0, « Conversation à la Ville Platte (Louisiane, Etats-Unis) : langue et musique en Louisiane », in Detey Sylvain, Durand Jacques, Laks Bernard \& Lyche Chantal (Eds), Les variétés $d u$ français parlé dans l'espace francophone. Ressources pour l'enseignement, Paris, Ophrys, 35I-364.

Morel Mary-Annick \& Danon-Boileau Laurent, 1998, Grammaire de l'intonation. L'exemple du français, Paris, Ophrys.

Neumann-Holzschuh Ingrid, 2004, « Le subjonctif en français acadien », in Brasseur Patrice \& Falkert Anita (Eds), Français d'Amérique : approches morphosyntaxiques, Paris, AIF et L'Harmattan (Coll. Langues et Développement), I25-I 44.

Pagliano Claudine \& Le Gac David, 20ı, « Conversation à Ogéviller (Meurthe-etMoselle) : les enfants d'hier et d'aujourd'hui, l'éducation se perd », in Detey Sylvain, Durand Jacques, Laks Bernard \& Lyche Chantal (Eds), Les variétés du français parlédans l'espace francophone. Ressources pour l'enseignement, Paris, Ophrys, 7I-8I.

Poiré François, 2010, « Conversation à Belle-Rivière (Ontario, Canada) : la transmission du français en milieu minoritaire », in Detey Sylvain, Durand Jacques, Laks Bernard \& Lyche Chantal (Eds), Les variétés du français parlé dans l'espace francophone. Ressources pour l'enseignement, Paris, Ophrys, 325-337.

Poplack Shana, 2009, « Quelle langue parlons-nous ? », in Les Cahiers de la Fondation Trudeau, Montréal, Fondation Trudeau, I25-I47.

Prignitz Gisèle, 2007, Corpus PFC-BFA, <http://www.projet-pfc.net/base-de-donnees/ enquetes>

Queffelec Ambroise, 2006, « Restructurations morphosyntaxiques en français populaire camerounais : l'expression des modalités injonctives et interrogatives dans le discours rapporté », in Le français en Afrique, 21, 267-280.

Romaine Suzanne, 1984, « On the problem of syntactic variation and pragmatic meaning in sociolinguistic theory », in Folia Linguistica, 18/3-4, 409-437.

Rosier Laurence, 2008, Le discours rapporté en français, Paris, Ophrys.

Rossi-Gensane Nathalie, 2007, « Quelles unités syntaxiques pour l'oral ? », in Bulletin PFC, 7, 359-372. 
Rossi-Gensane Nathalie, 2010, « Oralité, syntaxe et discours », in Detey Sylvain, Durand Jacques, Laks Bernard \& Lyche Chantal (Eds), Les variétés du français parlé dans l'espace francophone. Ressources pour l'enseignement, Paris, Ophrys, $83-106$.

Rossi-Gensane Nathalie (à paraittre). Syntactic variation in spoken French, in Detey Sylvain, Durand Jacques, Laks Bernard \& Lyche Chantal (Eds), Varieties of Spoken French : a Source Book, Oxford, Oxford University Press.

Rouquier Magali, 2002, « Les interrogatives en 'qui/qu'est-ce qui/que' en ancien français et en moyen français », in Cabiers de Grammaire, 27, 97-I20.

Skattum Ingse, 2008, Corpus PFC-MAA, <http://www.projet-pfc.net/ base-de-donnees/enquetes>

Skattum Ingse, 20II, « Si jétais riche ... Constructions hypothétiques en français parlé au Mali », in Autour du verbe, Le français en Afrique, 26, Nice, ILF \& CNRS, 49-70.

Terry Robert M., 1970, Contemporary French Interrogative Structures, Québec City, Editions Cosmos.

Walker Douglass C., 2010, « Conversation à Rivière-la-Paix (Alberta, Canada) : la francophonie rurale dans l'Ouest canadien », in Detey Sylvain, Durand Jacques, Laks Bernard \& Lyche Chantal (Eds), Les variétés du français parlé dans l'espace francophone. Ressources pour l'enseignement, Paris, Ophrys, 339-350.

Weiner Judith \& Labov William, 1977, « Constraints on the agentless passive », Communication au $39^{\mathrm{e}}$ LSA Summer Meeting, Honolulu. 
Français et multilinguisme urbanisé 



\section{Le français dans le plurilinguisme urbain algérien : les jeunes en parlent}

Un des mérites et certainement pas le seul de l'école de Chicago, est d'avoir considéré la ville comme « un laboratoire social » (Coulon 1992) qui met en relief la covariance entre langue et société. Grâce à cette conception, la ville a permis à plusieurs chercheurs d'étudier ses multiples transformations (Bulot 1999, 2004, 2009, Calvet 1994) et d'approcher certaines catégories sociales qui y vivent tels les délinquants, les immigrants ... (Labov 1978, Githinji 2013, Ledegen 2007, Billiez \& Trimaille 2007, Bertucci 2003, 2010). Cependant, l'apport de cette école est incontestablement perceptible en méthodologie d'enquête (Mucchielli. 2009). En effet, après les années 40, les chercheurs associés à cette école ont consacré de grands volumes de leurs études aux techniques d'investigation en ville (Grafmeyer 1978, Grafmeyer \& Joseph 1990). Entre les études qualitatives et les études quantitatives, les chercheurs se sont fortement inspirés des méthodes ethnologiques et ont défini des techniques d'enquête parfaitement efficaces quant à l'analyse profonde des pratiques langagières telle l'observation participante (Blanchet 2000, Laburthe-Tolra 1996). Suite à ces renouvellements, les nombreux travaux consacrés à la méthodologie de recherche en sociolinguistique mettent l'accent sur les procédés et les protocoles d'enquêtes, et sur les différents outils d'investigation susceptibles de rendre la collecte des données, dans le milieu urbain, plus sûre et plus fiable.

En Algérie, la ville a toujours été un corpus patent. En anthropologie (Hannerz 1980) et en sociolinguistique urbaine (Morsly 1996, Taleb Ibrahimi 2002, 1996), les chercheurs tentent d'accéder à cet espace dans le but de comprendre les multiples covariances entre individu, langue et territoire. Les problématiques les plus évoquées sont celles qui se rapportent à l'appropriation de l'espace par les locuteurs (Bulot 2009, Billiez 2013), et les différents discours épilinguistiques que ces derniers tiennent sur les lieux qu'ils occupent (Hedid 2013). L'épanouissement de ces travaux atteste de la richesse des approches épistémologiques et des démarches méthodologiques. 
Le plurilinguisme urbain tel qu'il est perçu et vécu par les chercheurs se pose comme une question cruciale, souvent porteuse de débats, voire de conflits. La cohabitation de plusieurs langues dans la ville algérienne rend le contexte urbain local plurilingue et difficile à étudier. L'arabe, le français et les autres codes en présence ne bénéficient pas des mêmes positions et, pour appréhender cette question, les scientifiques tentent d'adapter leurs méthodes et d'approcher ces interrogations sous plusieurs angles. Dans le présent travail, nous essayons de faire le point sur cette question, en mettant l'accent sur les méthodologies d'enquête utilisées pour cerner la place du français dans le plurilinguisme urbain algérien (Hannerz 1983). L'idée est d'actualiser les schémas descriptifs et méthodologiques déjà établis (Morsly 1996, Taleb Ibrahimi 1996, 2002, 2004) concernant la situation sociolinguistique du pays, et de voir la gestion que font les jeunes urbains d'aujourd'hui du potentiel linguistique qu' ils possèdent. La recherche repose sur une enquête de terrain dans la ville de Constantine et sur un corpus hétérogène. Notre étude tâchera de répondre aux questions suivantes : Quelle est la place qu'occupe le français dans le plurilinguisme urbain algérien ? Quelle méthodologie adopter pour aborder la question auprès des jeunes Algériens d'aujourd'hui ? Les travaux effectués dans ce sens se basent généralement sur des enquêtes de terrains où l'enquêteur observe ou enquête auprès d'un ou de plusieurs groupes dans un contexte bien déterminé. Nous essayons dans la présente recherche d'enquêter auprès du même groupe dans plusieurs contextes socio-discursifs pour mieux observer les changements linguistiques chez des informateurs (Lamarre 2009). Nous espérons, en fait, que cette filature ethnographique (Coulon 2009) portera des résultats plus précis sur l'état du plurilinguisme que connaît la ville algérienne aujourd'hui.

\section{Le français, les jeunes et la question sociolinguistique en Algérie}

La situation sociolinguistique de l'Algérie est caractérisée par une complexité apparente. Les chercheurs (Morsly 1987, 1993, 201 I, Taleb Ibrahimi 1997, Grandguillaume 1997, 2003) évoquent un plurilinguisme assez particulier, 
où sont présents plusieurs codes linguistiques, notamment l'arabe (avec plusieurs variantes), le tamazight (avec plusieurs variétés) et le français. D’autres langues sont aussi attestées dans certains secteurs tels l'enseignement, la recherche, la santé. Dans les pratiques langagières, les sociolinguistes relèvent un épanouissement remarquable (Cherrad 2004, Hedid 20II), les langues se mélangent (Caubet 2002), s'entrecroisent, et parfois l'une s'efface en cédant la place à un sociolecte nouveau. Le mécanisme est assez fréquent dans les contextes urbains. L'émergence de nouveaux parlers (Thiam 199I, Manessy 199I) est le résultat de l'urbanisation accélérée que connaissent les villes aujourd'hui et le plurilinguisme frappant que les locuteurs tentent d'assimiler. Les jeunes constituent une des catégories sociales les plus sensibles, et les plus vulnérables à tout changement sociolinguistique. En Algérie, leur parler est conçu essentiellement pour représenter leur univers, leur identité et la perception qu'ils ont de leur société (Morsly 1996, Taleb Ibrahimi 1996, Hedid 2011, 2013). L'étude de ce parler montre qu'il est composé de plusieurs langues, mais le français intégré est une des composantes de base (Morsly 1996, Hedid 201I). Nous essayons, dans la présente étude, d'adopter une autre approche et une posture différente de celles des autres recherches, dans le but de voir la place que le français tient dans le plurilinguisme urbain dans lequel vivent les jeunes Algériens.

\section{Le français dans le plurilinguisme urbain : méthodologie d'une enquête en mouvement}

Le « Tracking », comme l'appelle D.H. Zimmermann, est une méthode d'enquête qui s'inscrit amplement dans une perspective ethnographique. C'est en fait une étude micro-sociolinguistique qui se base avant tout sur une observation minutieuse des terrains d'enquête et des pratiques des groupes de locuteurs qui se définissent dans une certaine homogénéité. Évoquant cette démarche, A. Coulon explique qu' : « il s'agit de montrer, à des niveaux d'analyse variés, comment une structure se construit, par exemple comment en se fondant sur l'analyse des interactions concrètement échangées par les participants de l’activité scolaire, 'les activités 
structurantes de la classe assemblent les structures sociales de l'éducation' » (Coulon 2009: 82). Sur le plan conceptuel et théorique, les chercheurs expliquent que l'activité humaine tourne autour de quelques marqueurs sociaux dont l'identification permet l'organisation de la vie sociale (Durkheim I894). Ces marqueurs ne sont identifiables que dans le circuit des interactions (Vion 1992, Kerbrat Orecchioni 1990). Le passage par l'étude des interactions constitue dès lors un point important dans la compréhension de la présente problématique (Cavalli, Duchêne, Moore, Gajo, Marquilló Larruy, Matthey, Py, Serra 2009, Hedid 2013). Le protocole d'enquête se définit comme une enquête en mouvement (Lamarre 2009). Le principe de base de cette technique d'investigation : « consiste à observer le plus grand nombre de situations possibles au cours de la recherche sur le terrain » (Coulon 2009: 98). C'est une des méthodes d'enquête les plus fiables quant à la mise en relief des pratiques langagières et des changements linguistiques des locuteurs en synchronie. Les espaces socio-discursifs qui défilent offrent au chercheur la possibilité de suivre ses informateurs et de prendre des clichés sur toutes les situations d'interaction à laquelle ils adhèrent.

Ainsi le cadre épistémologique adopté ici se base sur une étude des pratiques communicatives d'un groupe de jeunes constantinois uni, homogène de plusieurs points de vue : lâge, leurs pratiques quotidiennes (sport), les études, les lieux fréquentés ...

Pour la sélection de ces informateurs, et prenant en considération le fait que notre démarche touche à la vie personnelle des informateurs, nous nous référons à une méthodologie labovienne : « qui fait appel au réseau personnel local de chaque enquêteur » (Durand, Lyche \& Laks 2005 : 208), c'est dire, comme l'explique P. Bourdieu qu': « on a ainsi pris le parti de laisser aux enquêteurs la liberté de choisir les enquêtés parmi les gens de connaissance ou des gens auprès de qui ils pouvaient être introduits par des gens de connaissance. La proximité sociale et la familiarité assurent en effet deux des conditions principales d'une communication 'non violente' » (Bourdieu et al. 1993 : 1395). Cette façon de procéder nous permet d'enquêter auprès des informateurs de notre entourage et de réduire toute contrainte relative au respect de la vie privée. Ainsi, les données analysées dans cette étude résultent d'une enquête menée auprès de deux de nos 
étudiants ${ }^{1}$. Les deux sont constantinois âgés entre I9 et 22 ans. Une fille et un garçon. Ces jeunes sont étudiants à l'université Constantine r et musiciens au conservatoire de la ville. En plus de ces points de ressemblances, les deux sont sportifs mais pratiquent des activités sportives différentes. La sélection de ces derniers était une étape très difficile. Nous avons sélectionné un grand nombre d'étudiants issus du département de français où nous sommes enseignante. Après l'explication du protocole d'enquête, beaucoup se sont désistés, expliquant qu'ils refusent d'être tout le temps contrôlés par l'enquêtrice. Les deux qui sont restés, ont montré un grand intérêt pour l'enquête et ont accepté de répondre à nos questionnaires et d'être suivis dans plusieurs lieux qu'ils fréquentent (sauf chez eux). Ces jeunes sont inscrits en première année licence de français. Hiba pratique la gymnastique dans une salle de sport en ville. Mohamed pratique le tennis dans un autre club, en ville, non loin de l'université.

Après avoir obtenu leur consentement, nous avons commencé à sélectionner les espaces propices à l'observation de leurs pratiques langagières. Nous avons été confrontée tout d'abord au refus des responsables du Conservatoire de nous laisser accéder à leur établissement afin de réaliser notre enquête, nous nous somme dirigée vers l'université, et grâce au fait que nous sommes enseignante, nous avons pu mener notre enquête avec les jeunes sélectionnés. Nous avons fait passer notre questionnaire, et nous avons confié notre magnétophone aux informateurs pour enregistrer, à tour de rôle, leurs rencontres avec leurs amis et leurs interactions en dehors des cours. Mais l'enquête dans les rues de la ville et dans les commerces était des plus difficiles : le bruit, la difficulté de suivre ces jeunes dans des quartiers commerçants surpeuplés à tout moment de la journée, ont rendu une grande partie de nos enregistrements inaudibles. Néanmoins, grâce à la prise de notes et à l'observation minutieuse de leurs échanges nous avons pu avoir un corpus intéressant.

Au total, nous avons sélectionné, en plus de l'université, les espaces suivants : la salle de sport de Hiba, et le stade où Mohamed joue au tennis, la rue, et quelques commerces que les jeunes fréquentent habituellement.

I Pour préserver l'anonymat de nos jeunes informateurs nous leur attribuons des pseudonymes (Hiba, Mohamed). 
La récolte des données était parfois pénible mais leur lecture est des plus intéressantes.

\section{Le français en ville algérienne, entre pratiques et représentations : les pratiques déclarées}

Le dépouillement et la lecture du premier corpus nous permet de dire que les jeunes enquêtés sont plurilingues. Effectivement, nous relevons pour la première question, une réponse commune, mettant l'arabe algérien comme la seule langue maternelle. Les deux jeunes ajoutent que leurs parents sont bilingues parlant deux langues : l'arabe et le français. À la troisième question qui concerne les langues étrangères pratiquées, les informateurs citent l'anglais et l'allemand. Quant au français, il semble complètement absent. Cette réponse, inattendue ${ }^{2}$, nous pousse à interroger les jeunes, qui nous disent alors que le français : « n'est pas une langue étrangère », Mohamed nous explique que : « le français est partout, moi je le parle toujours, il n'est pas comme l'anglais ». Quant à Hiba, elle semble étonnée qu'une enseignante de français à l'université lui pose une question pareille ; elle nous explique sur un ton ludique que le français n'est pas une langue mais : « un morceau de notre dialecte », et qu'en tant qu'enseignante algérienne je suis censée le savoir. Si le répertoire verbal semble plus ou moins homogène, les pratiques langagières telles qu'elles sont décrites dans les questionnaires laissent apparaître une certaine hétérogénéité.

Lorsqu'il s'agit de parler de ses pratiques langagières, Mohamed tient à expliquer que l'utilisation de telle ou telle variété est fonction de plusieurs paramètres. Trois codes sont cités par le jeune : l'arabe dialectal, le français et l'anglais. Il attribue au premier le statut de la langue « normale », dont la présence n’a pas à être expliquée, car elle est « la langue de tous les Algériens ». Le français tient une grande place dans les pratiques langagières 
des Algériens, selon Mohamed, donc sa présence est légitime et justifiable par le fait que « nous, les Algériens, même les plus arabophones, on ne peut pas parler sans prononcer ne serait-ce qu'un seul mot en français ». Avec ses frères et ses sœurs, il emploie ces deux codes, car ils sont, tout d'abord partagés, mieux encore, ils sont compris de tous. Avec les enseignants, la même alternance est relevée : Mohamed explique sa réponse par le fait que le français est la langue de ses études, il doit parler en cette langue avec ses enseignants mais il ajoute que l'arabe dialectal est aussi présent, employé aussi bien par les étudiants que par tous les enseignants sans exception.

Dans les autres cas, l'alternance des trois codes marque toutes les situations proposées. L'anglais tient ici une place très importante. Son usage semble être approuvé par le jeune, et sa présence véhicule des représentations perceptiblement positives. Mohamed nous explique que l'anglais, « bien qu'il était ignoré des Algériens, il devient aujourd'hui une langue pour la nouvelle génération ». Le jeune informateur tient à expliquer que la variété employée ici n'est pas celle de l'école ou des dictionnaires, mais celle de la télévision : « celle que les Américains parlent dans leur quotidien, comme je le vois sur MTV ». Pour illustrer, il nous cite les exemples suivants : « pour saluer on ne dit pas 'good morning', on dit 'hye', pour dire que je vais bien, je ne dis pas 'good', je dis 'meet', c'est plus jeune, c'est vraiment branché ». De même, la présence de cette langue n'obéit pas toujours aux mêmes conditions : à la maison, c'est surtout avec les parents que le jeune parle en anglais, non pas pour communiquer, mais pour formuler des messages codifiés que ces derniers ne comprennent pas, un usage à des fins parfaitement ludiques : « Lorsque je parle en anglais avec ma mère elle ne comprend rien et ça me fait rire ». Dans les situations plus formelles, comme l'université, la présence de l'anglais s'explique par le fait qu'il est une option dans le cursus universitaire de la licence de français. Mohamed nous dit qu'il l'étudie et qu'il s'intéresse au module, donc avec son enseignante et avec ses camarades cette langue constitue une langue de communication.

Hiba, la jeune fille, semble bien attachée à l'arabe dialectal et au français. L'alternance des deux marque toutes ses réponses, sauf à l'université et avec les enseignants où elle recourt à d'autres alternances. Lorsque nous lui posons la question pour comprendre son attachement à l'arabe dialectal et au français, elle nous explique que ce sont en fait : « les deux codes les plus 
maîtrisés par mon entourage. ». Elle ajoute que l'emploi d'un code ignoré des autres est un manque de respect envers eux. Ce qui est ici en question, concerne l'usage de l'anglais, Hiba affirme que cette langue marque surtout les situations de communication formelles: « je dois parler en anglais avec mon enseignante d'anglais et avec mes collègues, pour mieux m'entraîner ». L'informatrice explique par ailleurs : « J'essaye toujours d'employer un anglais très correct pour que je puisse m'entretenir avec les autres et qu'ils puissent me comprendre ». Le souci de parler correctement l'anglais est très apparent dans ses témoignages.

\section{Les pratiques réelles}

Dans chaque cas, nous sélectionnons deux espaces. La répartition faite dans notre corpus est due à deux facteurs :

I. L'activité sociale telle quelle s'organise dans ces différents espaces est différente, les participants à l'enquête modifient leurs comportements sociaux en fonction de ces configurations sociales.

2. Le changement de tout comportement entraîne une adaptation du langage et des pratiques langagières.

Nous présentons dans ce qui suit, l'analyse des corpus oraux enregistrés dans les différents espaces sélectionnés : l'université (les cours, les couloirs, cafétérias, ...), le stade de la ville (pendant les entraînements, vestiaires), la ville (la rue, les commerces).

Mohamed semble bien fidèle à ces trois langues. Les interactions enregistrées mettent en évidence son plurilinguisme. La lecture du tableau nous permet de relever les constatations suivantes :

I. Mohamed est plurilingue

2. Les deux variétés : français et arabe dialectal constituent une base solide de ce plurilinguisme, 
3. Le français et l'arabe dialectal sont sollicités dans les situations d'interaction formelles. Dans les séances de cours à l'université, Mohamed emploie l'arabe dans ses réponses aux questions :

ENSEIGNANTE : quelle est la différence entre un rapport et un compte-rendu ? MOHAMED : kima ngoulou le rapport est plus personnel, je peux mettre mes impressions (Trad. c'est comme on dit)

Avec son entraîneur, le jeune fait la même chose, quoique dans cette situation, nous remarquons que l'entraîneur lui-même recourt à l'arabe dialectal et les jeunes le suivent :

L'ENTRAÎNEUR : vous faites attention la prochaine fois, manhabech tmaskhir, ce n'est pas comme la plongée, vous n'êtes pas à Skikda (Trad. je n'aime pas le manque de sérieux)

MOHAMED : la dernière fois, les autres darouha masra oualou (Trad. Les autres l'ont fait, et il ne s'est rien passé)

Dans les autres situations, plus informelles, Mohamed emploie quatre variétés. Les interactions enregistrées montrent que l'usage de l'anglais et de l'arabe standard relève d'un emploi ludique, et s'intensifie une fois que le jeune est en face d'une personne de son âge :

Extrait $n^{\circ} I$ : Interaction dans les couloirs, pendant que les étudiants attendent l'enseignante MOHCENE : $\underline{\text { salam (Trad. la paix) }}$

MOHAMED : sahit, how are you men (Trad. salut)

MOHCENE : nes tgoul alikoum salam (Trad. les gens répondent: que la paix soit sur vous) MOHAMED : sur vous le peace (Trad. sur vous la paix)

Extrait $n^{\circ} 2$ : Interaction entre Mohamed et un groupe de filles

MOHAMED : ana rajl, mech kima ntouma, ana mina noukhba (Trad. je suis un homme, je fais partie de l'élite)

ESMA : alors là, aleh, ala fhamtek (Trad. alors là, pourquoi, pour ton intelligence ?)

NOUSSA : ghir skout, hata rabi meysmach (Trad. tais-toi, pour que dieu ne t'entende pas) MOHAMED : tarfou elrijel kaouamoun ala nisa (Trad. vous savez que les hommes sont supérieurs aux femmes)

FILLE2 : kamelha, oula tehfed ghir li yrongik (Trad. termine le verset, ou tu n'apprends que ce qui t'arrange) 
Dans les deux extraits le jeune mixe harmonieusement plusieurs codes : le français, l'anglais, l'arabe dialectal, standard et classique. Dans ce cas, le jeune s'entretient avec deux de ses camarades. L'interaction se déroule sur un ton ludique, l'emploi de l'arabe standard et de l'arabe classique revêt une conception plus distinguée : en effet, Mohamed les met sous forme de citation. En ville, aussi bien dans les rues que dans les commerces, Mohamed recourt à l'alternance arabe / français. L'anglais, l'arabe standard et l'arabe classique, par contre, sont complètement absents. Cet usage confère aux deux codes le statut de langues « passe partout », un usage simple et rentable qui rend efficace toute communication interpersonnelle urbaine.

Chez Hiba, les enregistrements sur les bandes sonores nous confirment son attachement à l'arabe dialectal et au français. Ces interactions reflètent ses réponses sur le questionnaire. Avec les enseignants pendant les séances des cours, la jeune fille n'utilise que le français.

HIBA : dans plusieurs situations, le dossier professionnel diffère selon les entreprises et les offres d'emploi.

L'ENSEIGNANTE : en Algérie ? pourquoi tu dis ça ?

HIBA : la dernière fois, on a demandé à mon frère de présenter le relevé des notes de toutes les années d'études à la fac, il était choqué !

L'ENSEIGNANTE : c'est généralement des entreprises privées

En classe les interventions de Hiba se multiplient dans les travaux dirigés (TD), où il s'agit de passer à la pratique et à la rédaction. La jeune informatrice monopolise la parole, elle s' impose de façon très claire. Les interactions de Hiba en classe (qui ont été effectuée de façon très discrète, les jeunes ne savaient pas que les cours étaient enregistrés) répondent parfaitement à celles de son enseignante. Le français domine les échanges, il est la langue enseignée et celle de la transmission des savoirs.

Sur le campus de l'université et dans les vestiaires de la salle de sport, Hiba mixe français, arabe dialectal et anglais. 


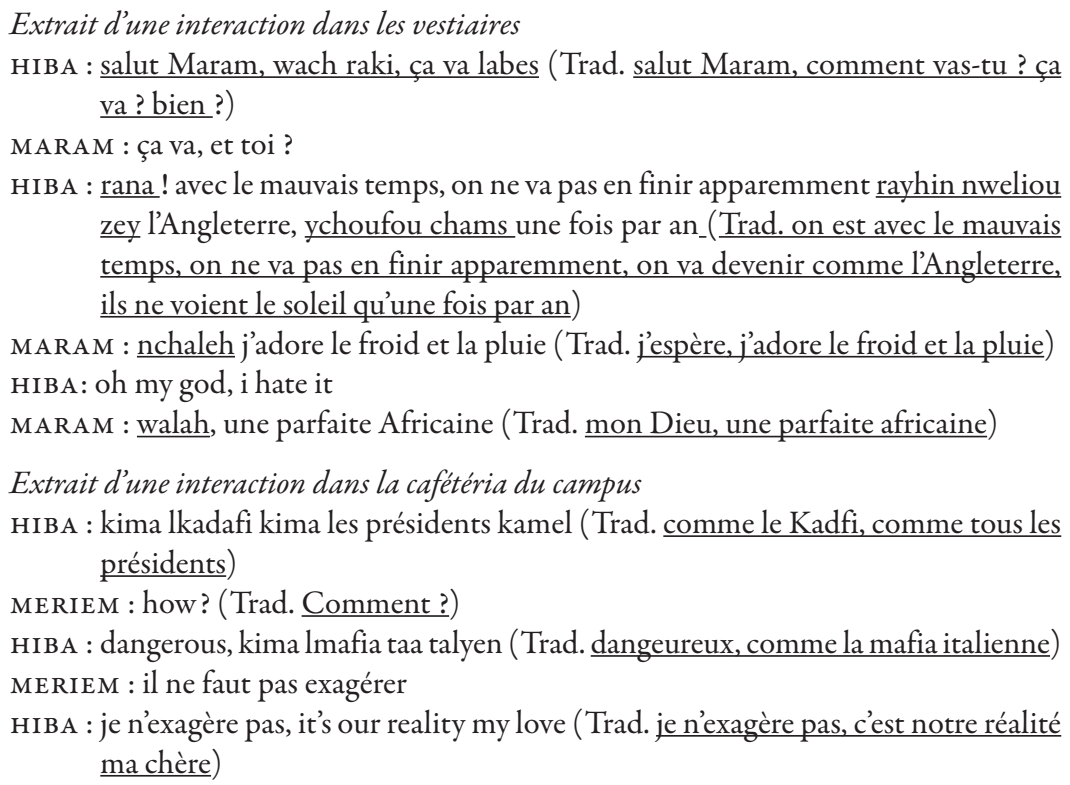

Hiba recourt à l'anglais pour répondre à son amie. Cette dernière semble vouloir déclencher à chaque fois des échanges en cette langue. Hiba la suit en employant des alternances avec l'arabe et le français. Toutes les langues mixées gardent dans ce cas leur structure, aucun code n'est encastré dans l'autre. Hiba continue, même dans ses interactions les plus informelles, à respecter la norme et à ne pas s'écarter de l'usage correct de l'anglais.

Contrairement à Mohamed, dans les rues de la ville, nous ne relevons qu'une interaction, celle de Hiba et une de ses voisines qu'elle aperçoit dans la foule des passagers. Ses rencontres sont souvent à la fac ou dans la salle de sport. Néanmoins, dans les commerces nous enregistrons quelques transactions avec les commerçants. Dans ces situations discursives urbaines les deux langues utilisées par l'informatrice sont le français et l'arabe dialectal, un choix qui confirme aussi bien l'attachement de Hiba à ces deux codes que leur domination dans l'espace urbain constantinois. 
La gestion du plurilinguisme chez les jeunes :

la place du français

La présente enquête confirme ce que la sociolinguistique variationniste a déjà mis en relief, à savoir l'influence qu'exerce l'âge sur les pratiques langagières. Les deux jeunes exposent, en effet, des comportements linguistiques différents de ceux des adultes : une appropriation des langues étrangères qui se manifeste de façon ludique dans leurs interactions à travers l'adoption de registres informels. Un plurilinguisme intégrant des codes linguistiques différents : l'arabe avec plusieurs variétés, le français, l'anglais. Cependant, ce qui semble important à noter dans nos corpus c'est la particularité du contexte sociolinguistique de la ville algérienne, et la gestion que font les jeunes du plurilinguisme urbain. Le français constitue une composante de base de ce plurilinguisme, il occupe une place importante et ce sur plusieurs plans. Nous essayons de définir dans ce qui suit quelques points de repères qui permettent de mieux saisir cette question :

\section{La maîtrise du français est un garant pour la réussite socioprofessionnelle}

Malgré une politique d'arabisation fortement soutenue par l'État, en Algérie, le secteur de l'emploi fonctionne encore (et ce dans plusieurs domaines) en français. Dans les entretiens d'embauche, dans les concours d'accès aux postes de travail, le français est considéré comme un critère de sélection. Les examens de français sont soumis à une évaluation rigoureuse, avec des coefficients assez élevés par rapport aux autres matières de spécialité.

La maîtrise de cette langue est très importante pour la réussite professionnelle; les jeunes ne sont pas insensibles à cette réalité. Dans leurs discours (sur leur répertoire verbal et sur les pratiques langagières), ils expriment clairement la nécessité de parler le français, en évoquant son importance pour trouver un emploi. 


\section{Le français n'est pas une langue mais un « dialecte »}

Un des arguments les plus employés par les jeunes pour expliquer la présence du français dans leur parler consiste à le considérer comme un dialecte. Ces informateurs nous expliquent le degré d'intimité qu'ils entretiennent avec cette langue et le fait qu'elle a intégré leur dialecte dans ses structures les plus profondes. Ces données poussent les jeunes à considérer le français comme faisant partie de leur dialecte. Ainsi, ils expliquent que certains vocables n'existant pas en arabe algérien, ne peuvent être désignés qu'en français.

\section{Le français n'est pas une langue étrangère}

Ce que les jeunes qualifient de langues étrangères sont les codes qu'ils apprennent à l'école, ceux qu'ils n'emploient pas souvent dans leurs interactions, et ceux qu'ils n'utilisent que dans certains espaces socio-discursifs (les entretiens d'embauches, les administrations,...). Le français semble dépasser ces frontières, il a intégré non seulement le quotidien de ces jeunes mais également leur imaginaire linguistique (Hedid 2014). Utilisé souvent en mélange avec l'arabe dialectal, il devient complètement algérianisé (Caubet 2009) et ce dans plusieurs séquences. C'est justement ce contact permanent avec les codes vernaculaires qui rend le français plus proche des jeunes et lui attribue en même temps le statut de langue locale « pas étrangère ».

\section{Le français est un «we code »}

Entre les jeunes, le français est utilisé comme un we code (Gumperz 1982) susceptible de garantir la solidarité de leurs groupes. Les différentes créations relevées et les déstructurations syntaxiques, grammaticales et morphosyntaxiques qu'ils font subir à cette langue sont en réalité des maillons de leurs réseaux qui garantissent une communication discrète. Le français devient dès lors un code à fonction cryptique (Bertucci 2003) qui permet 
aux jeunes de créer un espace d'interaction réservé à leurs pairs, aux jeunes de leur génération. En effet, le partage de certains vocables et l'usage de plusieurs expressions permettent à ces locuteurs de créer un espace communicatif clos, porteur de leur identité jeune et caractérisé par une solidarité très forte. Le français qui a marqué notamment les parents et l'ancienne génération, se voit aujourd'hui objet à de multiples transformations et plus encore, il devient un objet médiatique capable de véhiculer la pensée de ces jeunes.

\section{Le français a une place légitime, il peut pénétrer tous les espaces formels et informels}

Si certains codes ont leurs lieux d'apparition, s'ils sont réservés à certains espaces, le français semble extrêmement fluide et pénètre tous les espaces. Dans les contextes formels (l'université par exemple) ou informels (les stades, les commerces, ...) cette langue est présente. Son intégration dans les deux espaces n'est pas identique. Si dans les milieux formels, la présence du français est régie par des contraintes institutionnelles, dans les milieux informels elle est renvoyée aux compétences des locuteurs, des thèmes abordés,... Dans les espaces visités, dans les interactions enregistrées, le français s'impose parfois comme une langue support, dont la présence rend plus facile la compréhension et la fluidité des interactions verbales. Les multiples alternances que nous avons relevées lors de nos balades urbaines le montrent clairement. Cette langue est utilisée dans de nombreuses connexions avec les autres langues (l'anglais et l'arabe dialectal notamment), et semble posséder à chaque fois un rôle particulier et une fonction différente (il s'agit pour les jeunes de renforcer leurs dires ou de les traduire dans un français qui leur semble proche de leur réalité). Les fonctions ludique et cryptique permettent aux jeunes d'être plus proches, de renforcer leurs réseaux, de consolider l'apport sémantique de leurs échanges, dans tous les espaces discursifs qu'ils fréquentent. 
6. Le français est un code qui permet l'appropriation des territoires urbains, au même titre que l'arabe dialectal

L'appropriation des territoires est habituellement faite par le biais des codes vernaculaires et locaux (ou à une de leurs variantes) que les locuteurs emploient dans leurs interactions quotidiennes. Grâce à nos balades urbaines, nous remarquons que les jeunes utilisent notamment le français comme code d'appropriation des espaces occupés (Hedid 20r4a). Ce mode d'appropriation bien qu'il soit différent (car mobilisant une langue étrangère) permet à ces locuteurs d'imposer leur autorité et de prendre possession des différents territoires. Cette appropriation s'effectue par l'adaptation du français à l'espace visité, de le rendre plus fidèle au contexte sociolinguistique et culturel du site, et par la modification de certaines toponymies que les jeunes remplacent par des noms (souvent ludiques) et des créations telles que : « poubelle ville » à la place de « nouvelle ville » (pour évoquer hétéroclite de la Nouvelle ville de Constantine peu désirable pour les habitants de la ville), ou encore « Ali Congelé » à la place de «Ali Mendjli » pour évoquer le climat très froid de la région lors de la période hivernale. Dans la salle de sport ou dans les commerces, les échanges sont caractérisés par une accentuation de l'alternance des langues et par les créations lexicales, ces éléments se réduisent nettement lorsque les jeunes se trouvent dans d'autres sites tels que les couloirs de l'université ou les cafétérias du campus. Il devient plus facile de remarquer que dans chaque territoire, les jeunes tentent d'adapter leurs pratiques langagières pour les rendre plus caractéristiques des lieux qu'ils fréquentent.

\section{Même si les jeunes modifient leurs comportements langagiers, le français est toujours présent}

Le français est facilement maniable dans l'espace urbain. Lorsque les jeunes tentent d'adapter leur parler en fonction des contextes socio-discursifs, il reçoit les modifications nécessaires pour résister à ce changement, mais, il reste une composante principale dans leur plurilinguisme. Les modifications 
de leurs comportements langagiers, bien quelles visent la déstructuration de leurs habitudes linguistiques, ne marginalisent pas le français. Cette langue persiste et garde sa place, il semble évident quelle est une composante de base dans le répertoire verbal des jeunes, et dans le plurilinguisme urbain algérien.

\section{Le français assemble les idées}

Lorsque l'arabe n'est pas en mesure d'exprimer une pensée, le français intervient pour le faire. Dans plusieurs séquences, le français compense le manque, et permet aux locuteurs de communiquer sans que le manque de bagage linguistique (lexical) ne les interrompe.

\section{Que conclure?}

Il est clair qu'une enquête sociolinguistique en milieu urbain ne peut être que pluri-méthodologique. Un seul outil d'enquête ne peut mettre en évidence la complexité linguistique de la ville. Le recours à plusieurs techniques d'enquête (observation, enregistrement, questionnaire) a permis un meilleur cadrage des pratiques langagières des informateurs. De plus, l'adoption d'une filature ethnographique, et le suivi des jeunes dans leurs trajectoires quotidiennes dans plusieurs espaces en ville a donné des résultats intéressants. C'est, en fait, un renouvellement méthodologique que nous pensons utile pour appréhender la ville algérienne. Les jeunes urbains, dynamiques et actifs, sont souvent en déplacement, l'espace urbain leur offre la possibilité d'explorer des territoires différents. Donc pour aborder leurs pratiques langagières il faut adopter une méthode d'enquête qui s'adapte à leurs activités sociales. La technique, bien qu'elle ait rencontré plusieurs contraintes, a pu mettre à jour la complexité de la situation sociolinguistique de Constantine et la place qu'occupe chaque langue dans le plurilinguisme de ses jeunes locuteurs. 
Le travail sur la place qu'occupe une langue dans la mouvance du plurilinguisme urbain doit obéir à une méthodologie bien particulière. Si les locuteurs visés par notre étude se définissent par leur dynamisme, l'enquête doit suivre le même protocole. Elle doit capter les pratiques langagières et les représentations sociolinguistiques dans plusieurs sites qu'ils visitent.

Un des éléments sur lesquels les jeunes insistent est que le français ne peut être une langue étrangère, il a intégré leur parler et est devenu ainsi une partie de leur répertoire verbal. La place que cette langue occupe dans le plurilinguisme urbain algérien se définit avant tout par rapport à la place des autres langues dans le même circuit.

\section{Références}

Baude Olivier, 2006, Corpus oraux. Guide des bonnes pratiques, PUO, CNRS Éditions. Bertucci Marie-Madeleine, 2003a, « Le français des banlieues : un parler interstitiel ? », in Martin, Serge (Dir). Chercher les passages avec Daniel Delas, Paris, L'Harmattan, I33-139.

Bertucci Marie-Madeleine, 2003b, « Les parlers des jeunes en classe de français », in Le français d'aujourd'hui, I43, « Les langues des élèves », Paris, AFEF, 25-34.

Bertucci Marie-Madeleine, 2010, « Élèves migrants et maîtrise formelle de la langue de scolarisation : Variation et représentations », in L'intégration linguistique et éducative des enfants et des adolescents issus de l'immigration. Études et ressources $\mathrm{n}^{\circ}{ }_{3}$. Document préparé pour le Forum politique « Le droit des apprenants à la qualité et l'équité en éducation - Le rôle des compétences linguistiques et interculturelles », Genève, Suisse, $32 \mathrm{p}$.

Billiez Jacqueline \& Trimaille Cédric, 2007, « Pratiques langagières des jeunes urbains », in Molinarie Chiara et Galazzi Enrica, Les français en émergence, Bern, Peter Lang, 95-IIO.

Blanchet Philippe, 2000. La linguistique du terrain, méthodes et théories. Une approche ethno-sociolinguistique, Rennes, PUR.

Bourdieu Pierre, et al., 1993, La misère du monde, Paris, Seuil.

Bulot Thierry, 1999, « La production de l'espace urbain à Rouen : mise en mots de la ville urbanisée », in Bulot Thierry (Ed.), Langue urbaine et identité, (Langue 
et urbanisation linguistique à Rouen, Venise, Berlin, Athènes et Mons), Paris, L'Harmattan, 59-7I.

Bulot Thierry (Dir.), 2004a, Les parlers urbains. Pratiques urbaines et sociales, Cabiers de Sociolinguistique, 9.

Bulot Thierry (Dir.), 2004 b, Lieux de ville et identité. Perspectives en sociolinguistique urbaine, Volume I, Paris, L'Harmattan, Coll. « Marges linguistiques ».

Bulot Thierry (Dir.), 2009, Formes \& normes sociolinguistiques. Ségrégations et discriminations urbaines, Paris, L'Harmattan, Coll. « Espaces discursifs ».

Calvet Louis-Jean, 1994, Les voix de la ville. Introduction à la sociolinguistique urbaine, Paris, Essais Payot.

Canut Cécile \& Caubet Dominique (Dirs), 2002, Comment les langues se mélangent. Code switching en francophonie, Paris, L'Harmattan.

Cavalli Marisa, Duchêne Alexandre, Elmiger Daniel, Gajo Laurent, Matthey Marinette, Py Bernard, Serra Cecilia, 200I, « Le bilinguisme, représentations sociales, discours et contextes », in Moore Daniele (Dir.), Les représentations des langues et de leurs apprentissages, Paris, Didier, Coll. « Essais/Credif », 65-99.

Chapoulie Jean-Michel, 2000, « L'étrange carrière de la notion de classe sociale dans la tradition de Chicago en sociologie », in Archives européennes de sociologie, 4I-I, 53-70.

Chapoulie Jean-Michel, 200I, La tradition sociologique de Chicago, Seuil, Paris.

Cherrad Yasmina, 2004, « Paroles d'étudiants », in Des langues et des discours en question. Les Cabiers du SLADD, Université Constantine I, 25-43.

Coulon Alain, 1992, L'école de Chicago, Paris, PUF.

Coulon Alain, 2009, « La filature ethnographique », in Mucchielli Alex (Dir.), Dictionnaire des méthodes qualitatives en sciences humaines, Paris, Éditions Armand Colin, 97-99.

Durand. Jacques, Laks Bernard \& Lyche Chantal, 2005, « Un corpus numérisé pour la phonologie du français », in Williams Geoffrey (Ed.), La linguistique de corpus, Rennes, Presses Universitaires de Rennes, 205-217.

Durkheim Émile, 1894, Les règles de la méthode sociologique, Édition électronique.

Githinji Peter, 2013, « Linguistic competition in urban spaces : Sheng's value, utility and adaptation to Kenyan linguistic market », Conférence animée par (Université OHIO). Université de Cape town. Afrique du Sud à l'occasion du colloque international « African urban \& Youth language » (conference. AUYL).

Grafmeyer Yves, 2004, L'École de Chicago, P.U.G., Grenoble (I édition 1978).

Grafmeyer Yves \& Joseph Isaac (Ed.), 1990, L’école de Chicago. Naissance de l'écologie urbaine ( ${ }^{\circ}$ éd. 1979) Paris, Aubier.

Grandguillaume Gilbert, 1997, «L'oralité comme dévalorisation linguistique », in Peuples méditerranéens. Langue et stigmatisation sociale du Maghreb, 79, 9-I4. 
Grandguillaume Gilbert, 2003, « Arabofrancophonie et politiques linguistiques », in Glottopol, I, 70-75.

Gumperz John J., 1982, Discourse strategies. Cambridge (Mass.), Cambridge University Press.

Guth Suzie, 2004, Chicago 1920. Aux origines de la sociologie qualitative, Paris, Tétraèdre. Hannerz Ulf, 1980, Exploring the city: Inquiries toward an urban anthropology, Columbia University Press.

Hannerz Ulf, ${ }_{1983}$, Explorer la ville, Paris, Éditions de Minuit.

Hedid Souheila, 2010. « Le corpus urbain : un puzzle à reconstruire ». In Corpus entre donnée sociale et objet d'étude, Actes du colloque : « Corpus entre donnée sociale et objet d'étude », Université d'Alger, I27-137.

Hedid Souheila, 20Ira. Les représentations des parlers urbains chez les jeunes commerçants à Constantine. Le français comme marqueur social. Thèse de doctorat en cours, dirigé par Pr. Cherrad Yasmina et Pr. Bertucci Marie-Madeleine, Université Mentouri de Constantine.

Hedid Souheila, 2orıb, «Un français pour les jeunes Algériens », in Diversité. VilleÉcole Intégration, 164 , « La mer au milieu », Éditions CNDP-CRDP, 80-85.

Hedid Souheila, 20I3, « Lorsque les représentations sociolinguistiques redessinent la ville. La mise en mots de la mobilité socio-spatiale. Le cas de Constantine », in Glottopol, 21, Bertucci Marie-Madeleine (Dir.), « Lieux de ségrégation sociale et urbaine : tensions linguistiques et didactiques ? »,59-67, sur le site : <http:// glottopol.univ-rouen.fr/telecharger/numero_2I/gpl2I_04hedid.pdf>

Hedid Souheila 20I4, «Le LMD vecteur d'anglicisation en Algérie. Entre pratiques, besoins et représentations », in Hamez Marie-Pascale (Dir.), Langues Modernes, I, 2-6, <http://www.aplv-languesmodernes.org/IMG/pdf/20I4-I_hedid.pdf>

Hedid Souheila, à paraître, « La jeunesse sociolinguistique algérienne entre pratique et représentation », in Ebongue Augustin \& de Gratien Atingdogbe, Lesparlers jeunes du Cameroun. Éditions Peter Lang.

Kerbrat-Orecchioni Catherine, 1990. Les Interactions verbales, Armand Colin.

Labov William, 1967, Sociolinguistique, Paris, Minuit.

Labov William, 1978, Le Parler ordinaire, la langue dans les ghettos noirs américains, Paris, Minuit, 2 vol.

Laburthe-Tolra Philippe, 1996, «L'observation participante », in Mucchielli Alex (Dir.), 1996, Dictionnaire des méthodes qualitatives en sciences humaines et sociales, Paris, A. Colin.

Lamarre Patricia \& Lamarre Stéphanie, 2009, « Montréal 'on the move ' pour une approche ethnographique non-statique de l'étude des pratiques langagières de jeunes multilingues », in Bulot Thierry (Dir.), Formes \& normes sociolinguistiques. Ségrégation et discriminations urbaines, Paris, L'Harmattan, I05-I3I. 
Ledegen Gudrun (Ed.), 2007, Pratiques linguistiques des jeunes en terrains plurilingues, Paris, L'Harmattan, Coll. « Espaces discursifs ».

Manessy Gabriel, 1991, « Mode de structuration des parlers urbains », in Des langues et des villes. Actes du colloque International Dakar, Paris, Didier Erudition, 7-27.

Morsly Dalila, 1987, « Espaces de paroles: Pratiques et enjeux », in Espaces maghrébins : Pratiques et enjeux. Actes du colloque de Taghit, $\mathrm{I} 4 \mathrm{I}-\mathrm{I} 48$.

Morsly Dalila, 1993, « Les particularités lexicales du français parlé en Algérie », in Inventaire des usages de la francophonie nomenclatures et méthodologies, Paris, J. Libbey Eurotexte. 177-182.

Morsly Dalila, 1996a, « Alger plurilingue », in Plurilinguismes, I2, 47-80.

Morsly Dalila, 1996b, « Génération M6. Le français dans le parler des jeunes algérois $\gg$, in Plurilinguismes, $\mathrm{I} 2, \mathrm{III}-\mathrm{I} 2 \mathrm{I}$.

Morsly Dalila, 20ıI, «Enseigner la variation. L'exemple du Tamazight en Algérie », in Diversité, Ville-École-Intégration, I64, « La mer au milieu », CNDP-CRDP, I46-I5I.

Muchielli Alex (Dir.), 2009, Dictionnaire des méthodes qualitatives en sciences humaines, Paris, Armand Colin.

Peneff Jean, 2009, Le goût de l'observation, Paris, La découverte.

Taleb Ibrahimi Khaoula, 1996, « Remarques sur la parler des jeunes algériens de Bab El Oued », in Plurilinguismes, 12, 95-109.

Taleb Ibrahimi Khaoula, 1997, Les Algériens et leurs langues. Éléments pour une approche sociolinguistique de la société algérienne, Alger, Éditions El Hikma.

Taleb Ibrahimi Khaoula, 2002, « Entre Toponymie et langage, balade dans l'Alger plurilingue. Les enseignes des rues de notre ville », in Insaniyats, 17-18, 9-15.

Taleb Ibrahimi Khaoula, 2004, « Un cas exemplaire de métissage linguistique : les pratiques langagières des jeunes Algériens », in Dakhlia Jocelyne, Trames de langues. Usages et métissages linguistiques dans l'histoire du Maghreb, Paris, Édition Maisonneuve \& Larose, 439-354.

Thiam Ndiassé, 1991, « Nouveaux modèles de parlers et processus identitaires en milieu urbain : le cas de Dakar », in Des langues et des villes, Paris, Didier Erudition, 495-512.

Vion Robert, 1992, La communication verbale. Analyse des interactions, Paris, Hachette Supérieur. 


\section{Le français autrement}

Etre bilingue ou trilingue ou avoir la capacité de s'exprimer dans un nombre plus grand de langues signifie essentiellement une pensée qui s'exprime entre les langues. C'est un peu comme si l'on voyait le monde à travers les couleurs d'un arc en ciel. Loin de coïncider grossièrement avec les choses, comme c'est le cas lorsqu'une seule langue conditionne notre vision du monde, on arrive, avec cet écart que permet le plurilinguisme, à voir la réalité se décomposer et se recomposer autrement, à mesure que l'on navigue d'une langue à l'autre. Pour mieux appréhender ce concept, il suffit de considérer les déclinaisons du réel que permet le passage d'une langue à une autre dans le cas d'un simple mot, à savoir « lune ». Alors que le mot «moon » en anglais présente dans sa représentation graphique un redoublement de la pleine lune suggérée par l'aspect circulaire de la lettre « $\mathrm{O}$ », à deux reprises répétée, la lune déclinée en langue française acquiert, quant à la représentation graphique, une élévation différant du terme « moon » anglais, lequel s'étale plutôt horizontalement. De plus dotée intrinsèquement d'un déterminant féminin, voilà le signifié « lune » associé dans l'imaginaire de tout francophone au principe féminin. A l'encontre du signifié «moon » sans genre déterminé, que suggère l'usage de l'anglais, le terme « lune » en langue française évoque surtout les mutilations de la lune laquelle devient surtout cet astre qui décroît dans une association intime avec le féminin et les menstrues comme images isomorphes. Or pour l'arabophone que je suis, le mot « lune » français, une fois égrené en arabe s'avère différent. Le terme Qamar en arabe à l'opposé du terme français « lune » est du genre masculin. C'est que dans l'imaginaire collectif arabe, la lune ou Qamar est surtout le guide nocturne des caravanes qui dans l'immensité éperdue du désert se repéraient suivant la course de la lune. Dépossédée de cet aspect passif que lui confère, au niveau de l'inconscient, l'usage du genre féminin 
dans la langue française, la lune déclinée en arabe au masculin devient, dans l'imaginaire arabe, l'adjuvant par excellence des voyages millénaires à travers le désert. Le passage de la langue arabe à la langue turque dévoile pour moi une lune cette fois différente des trois précédentes abordées en langues anglaise, française et arabe. Appréhendé en langue turque pourtant si proche de l'arabe malgré l'alphabet latin, cet astre se présente aussitôt autrement. C'est quà l'instar de l'anglais, la langue turque n'impose pas à ses usagers un genre à déterminer comme c'est le cas pour l'arabe et le français. De plus l'usage du même mot en turc, à savoir « ay » pour désigner et la lune et le mois déclenche un passage à un autre niveau de la réalité appréhendée par le truchement de cette langue. La lune déclinée en turc évoque dans cette dualité lune / mois les temps immémoriaux où l'on adoptait en Orient le calendrier de l'Hégire ou un calendrier lunaire, les mois étant plutôt les douze lunaisons.

Le vieil Orient a toujours connu la grâce d'un plurilinguisme et d'une interculturalité avant la lettre, comme constante intrinsèque voire ontologique. Carrefour des civilisations et creuset des cultures, la Méditerranée, de laquelle on ne peut exclure un autre point cardinal : l'Est, autrement dit l'Orient, a toujours été non seulement plurilingue mais aussi un lieu de mémoire où persistent des langues immémoriales aujourd'hui tombées en désuétude comme c'est le cas du vieux syriaque de nos jours mis en pratique dans certains villages de la Syrie. De même des langues participant d'un passé révolu de la région, comme c'est le cas de la langue turque, demeurent un moyen d'expression pour les habitants d'un village situé au Nord du Liban. Vestige de l'époque ottomane, cette langue continue à faire partie de l'héritage culturel du Liban même après un siècle de la fin de l'Empire ottoman. Avant qu'elle ne dégénère de nos jours en espace d'achoppement entre diverses croyances, il est indéniable que la Méditerranée a assuré, dans l'Histoire, une fonction de lien entre Orient et Occident ainsi qu'un lieu de rencontre entre les civilisations humaines. Dans cette mosaïque de langues, de croyances et de coutumes, un repli identitaire était inconcevable, l'identité ne pouvant être réduite aux proportions étriquées d'un Même. La forclusion de l'autre, si forclusion il y a, s'effectuerait par conséquent au détriment du Même. Loin d'être un choc, la rencontre de l'autre faisait partie du paysage quotidien dans cette partie du monde où la réalité 
s'égrenait au fil des temps en langues et cultures diverses et où le rapport avec le Tout-Autre ou le Transcendant pouvait, à titre d'exemple, dans le même espace exigu, se faire simultanément, au fil des siècles, par le truchement des carillons et par la voix d'un muezzin sans que personne n'y trouve ni objet de surprise ni sujet de réprobation.

En tant qu'entité mosaïque, appartenant à un environnement qui n’a jamais été tout au long de son histoire ni monoculturel ni homogène, il n'est pas surprenant que le Liban, bien que sa langue maternelle soit l'arabe, ait adoptéle français comme langue d'expression. Aux ouvrages de Chékib Erslan, lequel, avec un certain nombre de ses contemporains au XIX ${ }^{\mathrm{e}}$ siècle, était influencé par la culture et la langue turques, a succédé au $\mathrm{XX}^{\mathrm{e}}$ siècle une kyrielle d'ouvrages où les écrivains libanais ont choisi cette fois le français et où paraît indéniable l'apport de la culture française.

Nous avons choisi de nous limiter vu l'espace qui nous est imparti à trois éminents écrivains de la francophonie libanaise dont deux hommes et une femme, à savoir Dominique Eddé, Salah Stétié à la fois poète et écrivain et Amin Maalouf. Loin de nous cantonner dans une analyse strictement littéraire, nous avons opté, à l'instar d'ailleurs de ces trois écrivains, pour un élargissement des frontières de notre réflexion, laquelle adopte le même élan de dépassement qui les a menés du littéraire vers l'anthropologique. Il est indéniable que l'adoption d'une langue autre ou de la langue de l'autre ne peut être abordée sans que ne soit soulevée aussitôt la question identitaire ou celle des cultures ou des civilisations selon le terme très en vogue dans le monde anglo-saxon.

Un simple tour d'horizon même hâtif de l'œuvre d'Amin Maalouf met le lecteur même inaverti devant un concept-pivot de l'écriture maaloufienne, à savoir celui de l'identité. Ayant vécu les affres de la guerre civile au Liban et souffert de l'hypertrophie de l'entité identitaire dans les deux camps dont l'opposition est fondée sur une démonétisation de l'altérité, Maalouf ne peut concevoir la problématique langagière qu'isomorphe de celle de l'identité par laquelle il paraît incessamment hanté : « J'ai constamment cité la langue au nombre des éléments qui définissent une culture, et une identité [...] » (Maalouf 1999 : 170). Or quand il s'agit de l'identité, elle se révèle selon Maalouf d'autant plus fétiche voire meurtrière qu'elle se conçoit monolithique, autrement dit exclusivement réduite « à une seule 
appartenance » (Maalouf 1999 : I4). L'individu se fige alors, comme le dit si bien Shayegan, dans une sorte «d'ankylose identitaire » (Shayegan 2008 : I4I) dans des « catégories trop étroites, que ce soient la nation, la tribu, le clan ou l'appartenance à une seule ethnie » (Shayegan 2008 : I4I). Effectuant une réflexion sur l'identité : «mon examen d'identité » dit-il, Maalouf semble se plaire dans l'affirmation de sa propre singularité. Loin de relever d'un noyau ou d'un centre fondateur « essentiel » (Shayegan $2008: 25$ ), qui marquerait la singularité d'un groupe auquel il appartiendrait, la différenciation réside, selon Maalouf, dans une identité multiple laquelle se révèle en outre inhérente à tout être humain. Dans une inscription permanente du multiple au sein de l'unique, tout homme se révèle, pour Maalouf, « foule » (Erri de Luca, Rez-de-chaussée, cité par Shayegan 2008 : I3I) du fait de ses appartenances multiples. Ne pouvant être que « composite » (Maalouf I999:30) c'est-à-dire multiple, l'identité s'avère d'autant plus unique qu'elle accueille en son sein la multiplicité : « plus les appartenances que je prends en compte sont nombreuses [écrit Maalouf], plus mon identité s'avère spécifique » (Maalouf I999 : 27). De même loin de se fixer sur la ligne immuable de l'être, l'identité relève du devenir : « l'identité n'est pas donnée une fois pour toutes, elle se construit et se transforme tout au long de l'existence » (Maalouf 1999 : 33). Maalouf, qui semble prendre plaisir à énumérer, comme autant de bénédictions, ses « appartenances » ethnique, religieuse et linguistique, paraît préférer au terme identité celui d'appartenance qu'il répète dans ses ouvrages : « [...] Je fouille ma mémoire [dit-il] pour débusquer le plus grand nombre d'éléments de mon identité, je les assemble, je les aligne, je n'en renie aucun » (Maalouf 1999 : 25). Se découvrant à souhait cosmopolite, Maalouf ne peut concevoir son identité que comme une «invention permanente » (Kaufman 2004 : 102) autrement dit ouverte : une identité-à-venir que l'homme « acquiert pas à pas » (Maalouf $1999: 35$ ).

Fondant la spécificité de l'identité sur la multiplicité des zones de mélange et de métissage ou «zone d'hybridation » (Shayegan 2008 : 60), Maalouf s'octroie dans Origines, une sorte d'identité-rhizome, sorte de dimension existentielle pour laquelle l'harmonie consiste dans le fait d'être à la lisière d'une multitude de mondes parfois incommensurables. Ce mode d'être s'avère l'un des vestiges de la vieille Méditerranée, dont 
les Libanais continuent à s'enorgueillir de nos jours. C'est en s'inspirant de cette « modernité-monde » (Shayegan 2008 : 44) avant la lettre qu'il se plaît à évoquer dans une sorte de mémoire récapitulative dans ses ouvrages - que Maalouf trace une sorte de « pédagogie réflexive » dont l'enjeu est un devenir-homme, au niveau de la planète, plus lucide. Dans notre civilisation ouverte où, du fait de l'interconnectivité, une multitude de niveaux de présence au monde tant archaïques que contemporains, se juxtaposent voire se contredisent au risque de s'annihiler l'une l'autre, « les hommes sont condamnés à se comprendre au risque de périr » (Shayegan 2012 : 46). Si une reconnaissance, enfin adulte, que les différences existent semble s'imposer de soi, cette reconnaissance s'avère loin d'être suffisante. Faisant de la différence le paradigme par excellence, Maalouf, dans un élan de dépassement de soi vers l'autre, préconise l'art de cultiver ses propres différences.

La diversité linguistique se révèle, selon Maalouf, l'adjuvant par excellence pour mettre en relation le Même et l'autre par delà un manichéisme ontologique et des représentations simplistes. L'écrivain prône ainsi la nécessité, devant laquelle se trouve désormais toute personne, à l'ère de la mondialisation, d'acquérir outre la langue identitaire et l'anglais ou la langue globale, une troisième langue que Maalouf appelle « la langue de cœur, la langue adoptive, la langue épousée, la langue aimée » (Maalouf I999 : I83). Cette langue-autre sera conçue comme un lieu de rencontre, une « passerelle », comme il se plaît à dire, « entre les diverses communautés » (Maalouf 1999 : 13), les diverses cultures. On se laisserait alors féconder par une langue et une culture autres que l'on enrichit à son tour soi-même par ses propres apports linguistiques et culturels. Si dans son cas, cette langue s'est avérée le français, cette langue-autre pourrait être pour un autre, affirme-t-il, « l'espagnol [...], le portugais, l'allemand [...], l'arabe, le japonais, le chinois, et cent autres langues pour lesquelles la spécialisation est plus rare [...] » (Maalouf I999 : 184); la langue française s'affirme d'autant plus la langue de prédilection de Maalouf, qu'elle s'inscrit dans une histoire et une culture avec toute la puissance d'un système symbolique qui, une fois né, d'une rencontre avec d'autres cultures, continue à s'inscrire sur ce même axe de mouvance où les rapports transculturel et interculturel s'avèrent un acte fondateur en permanence de la différence : 
il est certain que la langue française possède [...] une identité à multiples appartenances ; d'abord latine, oui, mais également germanique, celtique, avec des apports africains, antillais, arabes, slaves, ainsi que d'autres influences, plus récentes, qui l'enrichissent sans nécessairement l'altérer (Maalouf I999 : 207)

A l'opposé de cette notion d'une identité particulière que l'Orient n'a adoptée d'ailleurs que récemment, Maalouf rejoint une vision de l'identité en tant qu'un mode d'être de la coïncidence des contraires qui n'est pas sans rappeler certaines périodes illustres du vieil Orient. En clamant sa double appartenance culturelle : « mes deux univers culturels » (Maalouf 2009 : 33) et « l'autre civilisation que je dis mienne » (Maalouf 1999 : 36), Maalouf établit une nouvelle perception de l'identité associée à l'expérience spécifique dont jouit l'homme à conscience dédoublée. Cette « conscience métisse », plurielle ou « conscience des lisières » est maintes fois prônée par Maalouf. Se décrivant souvent comme étant « à la lisière de deux pays, de deux ou trois langues, de plusieurs traditions culturelles », Maalouf fait de cette conscience lucide d'une déchirure pleinement assumée l'espace identitaire par excellence ainsi que le fil d'Ariane pour une humanité-àvenir. Dans une sorte de brouillage constant de «l'originarité », Maalouf perçoit sa langue maternelle, à savoir l'arabe à l'origine comme déjà celle de l'autre alors que la langue-autre ou le français est en quelque sorte sienne. Son œuvre paraît dessiner une langue de la totalité, sorte de métalangue, qui paradoxalement ne peut se situer que dans un au-delà des langues ainsi qu'une métaculture autrement dit un au-delà des différences culturelles, qui les engloberait toutes.

Maalouf se révèle ainsi l'adepte d'une réflexion migrante où l'être humain se découvre ontologiquement « déterritorialisé ». Dans sa manière d'être soi, l'homme parvient à se regarder à travers le regard de l'autre. Ainsi dans la sarabande des personnages masculins peuplant l'univers romanesque de Maalouf : Tanios, Léon l'Africain, Mani, Baldassare, Ossyane, pour ne citer que ceux-ci, tous se révèlent ontologiquement des migrants à la fois d'ici et d'ailleurs, des rebelles aux liens et aux communautés étriquées. Comme l'enracinement s'avère quasi impossible, il n'est pas étrange par conséquent que le langage, quelle que soit la langue dans laquelle il est exprimé, ne peut, chez Maalouf, que désigner son inefficacité par rapport à un original inatteignable : «Une vie d'écriture m’a appris à me méfier 
des mots. Ceux qui paraissent les plus limpides sont les plus traîtres ». Et Maalouf d'ajouter : «L'un de ces faux-amis est justement 'identité. Nous croyons tous savoir ce que ce mot veut dire, et nous continuons à lui faire confiance même quand, insidieusement, il se met à dire le contraire » (Maalouf 2009 : 17). Maalouf cultive ainsi dans une sorte d'éclatement des signifiants, que dessine le dédoublement constant d'une conscience linguistique et culturelle réflexive, un art des nuances poussé à l'extrême. C'est par rapport à notre propre langue maternelle, comme par rapport à notre culture que nous sommes appelés à adopter de nouvelles herméneutiques, comme à considérer de nouvelles grilles de décodage, dont l'enjeu s'avère une prise de conscience, dit Maalouf, « du fait que [nos] propos ne sont pas innocents et qu'ils contribuent à perpétrer des préjugés qui se sont avérés, tout au long de l'histoire pervers et meurtriers » (Maalouf 2009: 32). Bien qu'il n'existe pas de formule magique pour notre ère, qui a atteint un niveau de dangerosité sans précédent dans l'histoire de l'humanité, Maalouf a recours cette fois à sa langue arabe pour en faire une esquisse. En substituant au mot français « formule », le mot arabe « sigha » (Maalouf 2009 : I88) cette formule, sorte de nouvelle herméneutique qu'Occident et Orient sont appelés à découvrir, est imaginée comme un travail d'orfevrerie nécessitant de part et d'autre, outre une grande patience, beaucoup de finesse.

Si pour Maalouf, le français est considéré comme « la langue de cœur » autrement dit la langue-autre choisie entre autres pour des raisons d'affinité, le français se dévoile dans les ouvrages de son compatriote le poète et l'écrivain Salah Stétié comme la langue de « l'Autre », lequel bien que situé aux antipodes de sa civilisation, s'avère le dépositaire de la Langue par excellence. Dans un article intitulé « Le Liban au cœur du français », Stétié décortique longuement ses rapports avec la langue française. Or cette réflexion débute par la notion de l'altérité. A chaque fois que Stétié explique son choix de la langue française comme langue d'écriture, ce que Maalouf ne fait pas d'ailleurs, l'image de l'Autre s'esquisse en filigrane. Le français est déterminé essentiellement comme la langue de l'Autre occidental qui, dès l'époque immémoriale de la route de soie, a fréquenté les chemins d'Orient : « Le rêve de soie palpite [écrit-il] depuis toujours comme une immense toile d'araignée sur l'univers des hommes. Pour elle, pour 
l'acquérir [...] ils sont allés de l'extrême Occident à l'Orient extrême [...]. Ils ont rencontré l'autre dans son étrangeté sidérale [...] 》 (Stétié 2008 : I3). Or « on parle [...] de l'Autre surtout quand on ne l'aime pas, qu'on en a peur ou quand il fascine » (Badie $1996: 237$ ). Dans le cas de Salah Stétié, l'Autre est bien celui qui l'a toujours fasciné. Voilà pourquoi la soie devient la métaphore de la langue française, ce qui l'amène à se décrire par conséquent comme un « produit soyeux » (Stétié 2008: I4) de cette langue, dit-il avec un sentiment de satisfaction bien explicite. Ayant fréquenté dès sa prime enfance la langue française, cette langue est devenue pour Stétié « la langue-mère ». Cette ascendance de l'autre s'accroît d'autant plus sur Stétié qu'il est question de sa mémoire blessée désormais en désaccord avec les grilles conceptuelles de sa propre culture. Au XX $\mathrm{XX}^{\mathrm{e}}$ siècle, écrit-il, « se défaisaient un peu partout les traditions et la culture de l'Orient ancien » (Stétié 2008 : 17). La langue française associée à la soie se révèle le corollaire d'une autre métaphore à savoir celle du papillon, à l'origine chrysalide, image du poète, qui, lors de l'adoption du français, s'octroie une origine en quelque sorte autre. Voilà pourquoi dans une comparaison qu'il établit avec son père, lui aussi poète, Stétié se réclame le fils de la langue de l'autre aux dépens de celle délaissée du père : «Mon père, qui était linguiste et grammairien, aimait passionnément sa langue qui lui tenait lieu de nourrice affective. Il me plaça pourtant, à quatre ans, entre les mains d'une autre nourrice qui me deviendra mère, cette langue française que je ne devais jamais plus quitter. Plus tard je rencontrerai comme si je l'avais écrit moimême et comme s'il célébrait ma propre mère, mère mentale s'entend, le fameux vers de Du Bellay : 'France, mère des arts, des armes et des lois' » (Stétié 2008 : 18-19). C'est ainsi qu’on assiste chez Stétié à la forclusion de l'arabe ; la langue française s'étant substituée à l'arabe comme seule langue identitaire. Loin d'être un processus d'accueil réussi de l'altérité, « l'hétérogenèse » ou « le devenir autre » (Shayegan 2012 : I4) est vécue par Stétié comme une hypertrophie de l'autre. Percevant ces deux univers, à savoir l'arabe et le français comme deux « blocs » de sensibilités si différentes qu'elles ne peuvent que s'exclure, Stétié adopte, pour « pouvoir passer à travers la grille conceptuelle moderne » (Shayegan 2008: 197) de la langue française, un « regard mutilé » par rapport à la sienne. Il confesse ainsi avoir passé une période de sa vie d'écrivain à trébucher en quelque sorte sur 
sa langue maternelle réduite désormais à une psalmodie du Coran ancrée dans la prime enfance à laquelle s'associent paradoxalement les chansons en vogue d'une célèbre chanteuse arabe :

Cette langue, dont vivent aujourd'hui près de trois cent millions d'Arabes et qui régit la vie religieuse et spirituelle de plus d'un milliard d'êtres humains - cette langue me sera restée jusquà mes années d'études à Paris, dans les années 50, une langue quasi étrangère, si l'on excepte l'enfance bercée par la psalmodie du Coran d'une part et, d'autre part, étrangement [...] - par la longue lamentation d'Oum Koulsoûm sur l'amour perdu. (Stétié 2008: 19)

Bredouillant sa propre langue dont l'usage s'est longtemps limité à « un dialecte [...] employé dans la pratique utilitaire » (Stétié $2008: 219$ ), Stétié semble privilégier dans l'hyper maîtrise de la langue française qu'il a fait sienne, un rapport extatique. C'est une relation fusionnelle qu'il établit avec le français sorte soit de langue-mère en qui il se love comme dans un cocon, qui lui permettrait, lui, la chrysalide d'opérer une totale mutation à l'image du papillon soit de langue-amante. Cette féminisation de la langue française est consolidée à maintes reprises dans les ouvrages de Stétié qui insiste d'ailleurs dans cet article sur la relation quasi charnelle qu'il instaure avec la langue française « aimée [dit-il] de moi par mon long accouplement avec elle, aimée de moi comme une personne peut être aimée, aimée d'amour, passionnément, éperdument [...] 》 (Stétié 2008:20).

Or sans rien nier de cette dimension féminine de la langue française dans laquelle Stétié s'inscrit comme sujet désirant, le voilà qui, malgré cette étreinte symbiotique, ne peut se départir à un moment de son existence d'un sentiment de désenchantement voire de malaise. C'est qu'on ne peut pas impunément faire le deuil de sa langue et de sa culture pour s'installer dans une sorte d'altérité imaginée de toutes pièces, où le Même est constamment relégué aux oubliettes. Dans cette obsession du « devenir-autre », du « devenir-étranger » suite à l'appropriation d'un espace discursif « autre », Stétié, du fait de sa jeunesse, aurait sous-estimé cette atrophie délibérée de l'ordre symbolique dans lequel ses origines ainsi que ses années formatrices ont été inscrites. Ce malaise apparaît sous la forme d'un sentiment de précarité ainsi que d'une mise en question radicale : 
C'est dans les années 50, sur les trottoirs du Quartier latin, face aux devantures des librairies regorgeant de livres intimidants - c'était alors la grande époque de Sartre, de Camus, de Malraux, de Merleau-Ponty, d'Eluard, de Breton, de Michel Leiris $[\ldots]$ - oui, c'est face à ces librairies [...] que, pour la première fois, je me suis demandé avec angoisse : «Qui suis-je ? 》 (Stétié 2008:19)

Ayant épousé la langue et les codes culturels de l'Autre comme s'ils étaient les siens, Stétié semble pour la première fois remettre en question les dangers d'une telle désappropriation où l'autre devient l'unique « donneur de sens ». Nous devons ici souligner que cette prise en considération de sa propre langue comme de ses propres codes culturels s'avère d'autant plus incontournable qu'il s'y voit amené, une fois encore, par le regard de l'autre ou les efforts conjugués de ses deux mentors qui bien qu'occidentaux, à savoir Gabriel Bounoure et Louis Massignon, se révèlent des figures emblématiques du génie universel : « Je savais par mon maître Gabriel Bounoure, je savais par mon maître Louis Massignon que ce n'était pas rien que d'être Arabe, que ce n'était pas rien que d'être d'Islam et que, bien au contraire, à partir de là, on pouvait se retrouver et se reconnaître à travers tous les signes fulgurants d'une immense civilisation capable de création et d'échange, d'ouverture et d'affirmation de l'inaltérable et de l'inaliénable, de synthèse et de symbiose, mais aussi de refus de compromis sur quelques-unes des questions essentielles où se trouvent engagés le destin de l'homme et le sens de son Dieu » (Stétié 2008: 19).

A «l'altérité intériorisée » (Shayegan 2008 : I83) à l'aune de laquelle il s'est exclusivement mesuré, le rapport avec le Même étant essentiellement faussé à moins d'être occulté, se substitue une nouvelle forme d'altérité laquelle découle d'un rapport dialogique instauré dorénavant entre les différents niveaux culturels qui, de par son statut de bilingue, conditionnent sa perception de la réalité. Recouvrant des productions culturelles relevant, pour la première fois, tout autant de l'Autre comme du Même, lequel ne peut plus être tenu pour totalement négligeable, cette altérité nouvelle nourrit la démarche comparative qu'effectue désormais Stétié. Dans la mesure où elle permet à l'écrivain de comprendre les différences entre les modèles de son propre développement culturel, elle paraît favoriser l'émergence d'une altérité fondée sur la comparaison et que nous pouvons saisir comme « altérité méthodologique intériorisée ». Stétié est alors à même 
de comprendre qu'un authentique avènement à l'écriture ne peut avoir lieu quà partir d'une identité individualisée c'est-à-dire à partir de son propre monde culturel, qui, quoique mutilé, persiste encore :

Je compris que j'étais Arabe [...] oui, je compris ce jour-là que l'Arabe que j'étais s'accomplirait en tant que Libanais et en tant qu'Arabe dans la langue d'en face, celle de l'autre rive de la Méditerranée. (Stétié 2008:20)

Stétié adopte dès lors une démarche inverse à celle menée auparavant. De « mutant 》 (Shayegan $2008: 226$ ) souffrant d'une « schizophrénie épistémologique » (Shayegan $1992: 278$ ) par rapport à tout ce qu'il personnifie, il se transforme en migrant doté comme le dit si bien Daryush Shayegan d'un « double regard qui le distancie et par rapport à la tradition à laquelle il appartient et des mutations culturelles dont il est porteur » (Shayegan 2008 : 230). Refusant d'être désormais le miroir de l'autre, Stétié passe à la contre-offensive mais néanmoins sans violence. Doté de tout ce que la langue arabe, cette « langue d'empire », ainsi qu'il la qualifie dans l'un de ses derniers ouvrages, véhicule comme codes culturels et symboliques, Stétié s'octroie le droit de refaire autrement la langue française qui a contribué à le faire : j’ai compris, dit Stétié, « que cette langue qui fut parfois celle de l'envahisseur, [...] il m'appartiendra bientôt, par mon œuvre (déjà je croyais à l'œuvre qui viendra [ajoute-t-il]) d'envahir à mon tour pacifiquement. Pacifiquement, oui, certes, mais sans rien renier de mes acquis ni rien abandonner de mon bagage » (Stétié 2008:20), tient-il à préciser. Cette double mise à distance fait de lui un maître dans l'art de la conversion : « J'entrais dans la langue française comme chez moi et le couvent qu'elle me paraissait parfois, je rêvais de le transformer en sérail, je veux dire d'adapter à ma propre structure intime les éléments d'un bâti imposé mais ductile et transformable » (Stétié 2008:20).

Dans cet entrecroisement des langues et des cultures, Stétié s'enorgueillit de ce qu'il transporte de sa culture dans la langue de l'Autre. S'il reconnaît « ce que la langue française lui donne », Stétié affirme par ailleurs, en « toute modestie » précise-t-il, que « ce qu'il donne de lui-même à la langue française, elle ne saurait le recevoir de quelqu'un autre que lui » (Stétié 2008 :20) à savoir un langage inédit, rendu possible justement par ce qui le distingue lui l'Oriental de l'autre occidental : 
Comme les rois-mages, les écrivains arabes de langue française $[. .$.$] sont porteurs$ naturellement de présents, et d'un ailleurs. Cet ailleurs, ces présents, c'est dans leur mémoire immémoriale qu'ils les trouvent et qu’ils les communiquent [...] à la langue de l'autre. Voir avec les yeux de l'âme est bien, selon Stétié, l'apanage d'un Orient, lequel, à l'encontre de l'Occident, n'a jamais consenti à déserter « le continent de l'âme » : « les écrivains arabo-francophones, adossés à la puissance historique et créatrice de la langue arabe, peuvent se flatter, à juste titre, d'étendre les limites de la francophonie jusqu’à des frontières qu’elle n'aurait pas pu conquérir sans eux. (Stétié 2008:29)

L'œuvre de Salah Stétié laisse affleurer des interrogations récurrentes où l'écrivain n'en finit pas de visiter et de revisiter les mêmes lieux : son choix de la langue française comme langue d'écriture de poésie, la vieille Méditerranée plurilingue et pluriculturelle et surtout sa grande douleur de voir l'Orient sombrer dans le feu et le sang. Néanmoins dans ses derniers ouvrages, Stétié paraît hanté par un héritage mystique oriental dont les noms les plus illustres sont le persan Hallaj, le turc Rumi et l'arabe-andalou Ibn Arabi. Ayant choisi la langue de l'autre pour rendre possible l'articulation de ce langage ontologique centré sur le Tout-Autre, Stétié accomplit ce que Shayegan nomme un processus de transmutation. Stétié conçoit le verbe poétique dans cette torsion du langage sur luimême qui, lors du choix d'une langue-autre, joue à merveille. Le poète Stétié s'embarque plus aisément dans « les contrées de l'être » de la mystique orientale dans la mesure où il s'ouvre « au jeu des influences » de la langue française et de la culture occidentale auxquelles il infuse « les mythes profonds et les inspirations dont [sa] conscience et [son] inconscient sont chargés $»^{1}$. S'il reconnaît dorénavant qu’il réside au cœur d'un Langage transcendant qui lui parle dans sa langue d'origine, Stétié, pour qui la poésie est un «langage hors du langage » (Brun 1985: 177), délivre grâce à cet entrecroisement des langues et cultures un murmure d'autant plus ontologique qu'il s'ancre dans un emboîtement de migrations : linguistique, culturelle et symbolique. Ancré de par la langue arabe au Mystère de l'Ipséité, Stétié trouve dans ce mode de dire qu'est la langue française son fondement sur la ligne du devenir. Aussi écrit-il : « La langue française est l'une de ces langues dont beaucoup d'hommes et de femmes, disséminés sur les cinq continents, ont besoin pour vivre, moi le premier » (Stétié 2008 : I5). 
Si l'œuvre de Maalouf et de Stétié exposent leur vision du plurilinguisme et de l'interculturalité s'interrogeant sur la pertinence de certaines notions comme celles de la culture et de l'identité, le roman de Dominique Eddé intitulé Cerf-volant laisse voir les derniers jours de ces pays de l'Orient qu'on appelle Le Levant, où survivait encore la grâce d'un temps révolu, sorte d'âge d'or. Des langues et des cultures variées étaient alors en interaction, exerçant une influence les unes sur les autres sans qu' il soit besoin de définir une pensée ou un vécu interculturels, lesquels existaient tout simplement comme partie intégrante de la vie quotidienne de ce Levant, bien avant que ces termes ne soient forgés par un Occident qui les a découverts à l'ère de la globalisation. Ce vieil Orient du XIX siècle revit sous nos yeux raconté par Eddé ainsi que par Mali le personnage féminin principal et la narratrice dans ce roman en abyme qui s'inscrit dans Cerf-volant d'autant plus que Mali tarde à l'écrire, et que Loulwa, son amie de longue date, insiste auprès d'elle afin que Mali consente à le rédiger. Mais c'est bien d'un roman du XIX ${ }^{\mathrm{e}}$ siècle qu'il s'agit : «Je n'arrive pas à comprendre pourquoi tu n'écris pas, Mali. Ne le prends pas mal, mais j'ai le sentiment quand je t'écoute parler de lire un roman du siècle dernier $\gg$ (Eddé 2003: 162-163). A maintes reprises, le roman met en relief cette survivance du XIX ${ }^{\mathrm{e}}$ siècle dans cette période de la première moitié du $\mathrm{XX}^{\mathrm{e}}$ siècle qu'évoquent les souvenirs de Mali, à savoir une Alexandrie avant Nasser, où « le raffinement et le parfum du passé [...] tenait sur les peaux » (Eddé 2003: 142), et un village d'été du Liban du nom emblématique de Sofar - «So far » écrit Mali - où persistait dans cette première moitié du $\mathrm{XX}^{\mathrm{e}}$ siècle un temps paradoxalement révolu, que la « mémoire épuisée » de Mali s'efforce de réanimer. De même le personnage masculin principal, Farid, l’amant de Mali, est surtout attiré par « son côté dix-neuvième » (Eddé 2003:25I) : «Dans le fond, Mali a toujours vécu dans une autre époque » (Eddé 2003:252) affirme-t-il. C'est effectivement d'une autre époque que traite le roman voire d'un autre Orient que Loulwa et Mali s'enorgueillissent d'avoir connu et s'évertuent tout au long du roman à évoquer : « C'est vrai que tout a foiré dans nos pays ... mais nous avons quand même eu de la chance, quand j'y pense, beaucoup de chance [...] » (Eddé 2003 : 18-19) dit Loulwa, cette sorte d'âme-sœur de Mali.

Beyrouth « ville cosmopolite » (Eddé 2003 : 142), avant que la guerre civile de 1975 ne signe « sa mise à mort » (Eddé 2003 : 224), se révèle, 
dans ce roman, la métaphore d'un vieil Orient où il faisait bon de penser et de vivre une « interculturalité » avant la lettre. Occupant la position d'intermédiaires, voire de passeurs, les personnages d'Eddé naviguent entre les langues, entre les cultures, comme entre les religions. Dans ce Grand Hotel de Sofar, les gens « parlaient en arabe ou en français et parfois dans d'autres langues [...]. Leurs phrases étaient [...] fleuries de noms aux couleurs vives, des prénoms cueillis aux quatre coins du monde, dans le Coran, les Evangiles, les romans anglais et russes du siècle dernier, les livrets d'opéra, les chansonnettes, les films, les gouvernements français » (Eddé 2003: 43). Il n'est jusqu'au prénom de Mali qui, lui aussi, est décliné, dans le roman, en plusieurs langues : Maliha (Eddé 2003 : 133), Malou et Malouchka (Eddé $2003: 38$ ), dans une allusion symbolique à cette particularité du vieil Orient où, du fait de son ouverture à d'autres mondes, l'on « s'identifi[ait] à soi en train d'être dés-identifié ». Dans cette « non-coïncidence à soi » dont parle Sartre, qui, dans le cas du vieil Orient, relève d'un rapport permanent du Même avec l'Autre, en d'autres termes d'une altérité inhérente à la réalité quotidienne, on assiste parfois à une sorte de prolifération comique sinon monstrueuse de l'autre comme c'est le cas, écrit Eddé, de cette dame, qui, « dans un français plus que parfait [énonce] : Notre arabité est incontestable' » (Wirman 2012:39).

Loin de se limiter aux proportions d'un roman auto-fictionnel, où il s'agit d'effectuer un va et vient constant entre le passé et le présent, Cerfvolant pose la question de la lutte à laquelle se livrent le Même et l'Autre. Dans ce roman à plusieurs niveaux symboliques, c'est bien de la relation entre l'Orient et l'Occident voire de la nécessité d'une communion entre ces deux entités qu'il est question. Or cette réflexion, qui, dans le roman d'Eddé, semble avoir pour enjeu toute l'humanité (l'Orient et l'Occident, les pays du Nord et ceux du Sud, les Arabes et Israël) s'effectue essentiellement à partir de la grille de la langue, en l'occurrence l'arabe, le français et l'anglais. Il est intéressant de souligner que dans leurs rapports aux « trois langues, arabe, français, anglais (superposées [...] dans nos têtes) » (Eddé 2003 : 49), écrit Mali, cette dernière ainsi que Farid ne peuvent se départir d'une perspective comparative où la langue signifie sans que quelqu'un la parle (Humboldt cité par Weismann 20I2: 72) au sens qu'elle revêt « une individualité collective » (Humboldt cité par Weismann 2012 : 72) Le 
processus d'individuation des deux amants que trace le roman ainsi que l'Histoire du Moyen-Orient s' inscrivent dans une sorte d'histoire commune de ces trois « individualités collectives » : l'arabe, le français et l'anglais, qui, de par leur essor ou de par leur déclin, semblent façonner le destin non seulement de Mali et de Farid mais aussi de toute la région.

Cette conception de la problématique des langues comme pierre d'angle du roman est consolidée dès les premières lignes où il est question d'un quidam, lequel « se vantait de connaître une dizaine de langues dont l'amharique et le tagalog » (Eddé 2003 : II). Farid est présenté comme un fin connaisseur des dialectes arabes et de l'hébreu, ce qui lui a valu d'être nommé « directeur du département de philologie à l'université d'Oxford » (Eddé 2003 : 15). Quant à Mali, c'est en grande partie parce qu'elle n'arrive pas à se décider entre l'arabe et le français qu’elle n'arrive pas à écrire son roman. Toujours est-il que les personnages de Dominique Eddé sont exposés, de par « le foisonnement de leurs appartenances, à des groupes tous porteurs de cultures particulières $\gg$ (Verbunt 20II : 69). Mali est ainsi de père libanais et de mère française. De même Farid est un musulman dont la mère a été élevée par une grand-mère chrétienne qui, bien qu’arabe, était affublée du nom de «Christmas ».

Aussi peut-on avancer qu'il s'agit d'êtres qui se cherchent en quelque sorte dans les langues comme c'est le cas de Farid dont la réflexion politique est le corollaire d'une prise en considération de l'altérité linguistique : « Ses travaux sur l'arabe et l'hébreu donnaient une dimension supplémentaire à sa réflexion politique » (Eddé 2003 : 187). De même c'est à partir des mécanismes implicites d'une langue, en l'occurrence l'arabe, qu'il se propose de réfléchir sur les problèmes actuels du monde arabe. Aussi intitule-t-il ses ouvrages : «La langue arabe et le temps » (Eddé 2003: 229), ainsi que «Words and double bind » (Eddé 2003: 248).

Quant à Mali, c'est entre deux héritages linguistique et culturel, à savoir le français et l'arabe qu'elle se débat pour pouvoir accéder au monde de l'écriture. En désaccord avec son double héritage, Mali fait jouer ses deux univers linguistique et culturel l'un contre l'autre: « [...] c'était en réalité ses deux langues - l'arabe et le français qui souffraient chacune à sa manière de la même censure et de la même tyrannie » (Eddé 2003 : 6I). Concevant ses deux langues comme « deux jardins opposés » (Eddé 2003:52), la voilà qui 
se met dans l'impossibilité d'écrire en langue arabe, désormais « en cage » (Eddé 2003: 60) car Mali est hantée par le « souvenir des lettres latines [lequel] brisait le rythme et l'ouverture des lettres arabes qui, couchées ou debout, se heurtaient à des frontières qui n'étaient pas les leurs » (Eddé 2003 : 57). Habitant la langue maternelle, Mali entretient avec le français des « rapports tordus » (Eddé 2003: 160), dit-elle. En effet si cette langue se révèle pour Mali « un objet de vénération » (Eddé 2003:56), elle n'est pas pour autant un objet d'amour (Eddé 2003: 56). L'oxymore qui définit sa perception contrastée de la langue française, à laquelle elle voue « une passion froide », met en évidence l'attrait qu'exerce cette langue sur Mali. L'exil linguistique voire le mutisme, auquel se contraint Mali incapable de s'exprimer correctement en arabe du fait qu'elle ne maîtrise pas cette langue et refusant d'écrire en français; langue avec laquelle elle instaure une relation du rejet sinon d'hostilité, s'expliquent surtout par un rapport défectueux quelle établit à l'origine avec l'Occident, en la personne de sa grand-mère maternelle. Ayant renié sa fille Claire, ou la mère de Mali, pour avoir choisi l'Orient, la grand-mère maternelle devient pour cette dernière l'emblème de l'ipséité autonome d'un Occident fermé à toute communion. Définie par Bataille comme « la pénétration d'un être par un autre » (cité par Weismann 2012 : I17), la communion s'avère quasi impossible, dans le cas de la grand-mère, du fait d'une identité monolithique, laquelle constitue « une véritable prison de l'esprit comme de l'affect 》 (Weismann 20I2: 50) : Mali « se bornait à détester cette femme qui, repliée sur ses titres et ses terres, avait si bien survécu au triple deuil de son mari, de son fils et de sa fille. Et, dans l'emportement de la colère, il lui arrivait souvent de confondre la France, toute la France, avec cette vieille dame avare, élégante et glacée $\gg$ (Weismann 2003: 50).

L'Occident a été longtemps perçu par Mali sous les trait d'un « Même qui écrase toute altérité » et par conséquent stérilise, d'où son incapacité d'écrire. C'est au sème de la stérilité voire de la mort, que Mali associe l'autre. Hantée par la proximité de la mort du fait que « sa mère avait peut-être payé de sa vie la vie de son couple » (Weismann 2003:6I), Mali, pourtant à moitié française, nourrit vis-à-vis des Occidentaux un profond sentiment de rancune. Aussi fait-elle dire à Danse Vavikian, l'un de ses personnages et lui-même romancier dans cet emboîtement de romans en abyme qui 
sécrivent dans Cerf-volant: « Ils trouvaient normal qu’on parle leurs langues et ils ne trouvaient pas normal qu'on ait des choses à dire » (Eddé 2003 : 32). Traitant les Occidentaux de « donneurs de leçons » (Eddé 2003 : 30), Vavikian, sorte de double psychique de Mali, paraît leur reprocher à l'instar de la narratrice ce qu'elle appelle leur « arrogance » (Eddé 2003 : I87). Comme prototype de l'Occidental qui «s'interpose lui et sa culture comme une mesure et un plus sûr critère des choses » (Weismann 2012 : 296), l'un des amis de Mali mentionne « Rosetta Fadalli », une Italienne, dit-il, qui « a fait deux séjours d'une semaine à Damas et au Caire et est sur le point d'achever un livre intitulé Où va le monde arabe » (Eddé 2003 : I92). A cette image se superpose dans Cerf volant, celle de deux serveurs dans un café de Paris, prototypes d'une certaine «Europe frileuse » car confrontée à une mondialité qui la dépasse. En effet déconcertés par ces nomades culturelles et linguistiques que sont Mali et Loulwa, ils expriment à leur égard une méfiance simpliste propre aux sédentaires bien « enracinés » dans leur « tradition monoculturelle ( Verbunt 20II : 27) «Tu as vu ces deux femmes? Elles sont incroyables » dit le serveur à son collègue. «La plus jeune des deux a plus de soixante-cinq ans, tu as vu comme elles rigolent ? On dirait des gamines. Elles parlent en français et parfois en arabe [...] elles [parlaient] politique et pourtant elles riaient tellement qu'elles se suppliaient l'une l'autre de se taire ». Et l'autre de répondre : « Ecoute, je ne suis pas raciste mais je commence à en avoir assez de ces Arabes qui sont partout chez eux ».

Confrontée à cette image de l'Occident qui a admis une fois pour toutes le primat du Même sur l'Autre, Mali imagine la langue française comme « un lieu de résistance, une tranchée » (Eddé 2003 : 192). A cet espace linguistique belliqueux imposant une certaine clôture de l'être, s'oppose, dans Cerf-volant, la langue arabe qui, du fait qu'elle s'inscrit dans un ordre où la différence joue au cœur même de l'identité, marque un net désir de différenciation et d'ouverture :

En arabe, où chaque lettre est un mot et chaque mot, ou presque, une branche née d'une racine de deux ou trois lettres, toute phrase est une poussée de sève à l'intérieur de la langue, toute pensée, le rayonnement circulaire d'un arbre invisible, membre tantôt sain, tantôt pourri, d'un jardin de signes. (Eddé 2003 : 58) 
Formés à partir d'une racine, les mots en arabe, du fait d'une racine partagée et de sens différents, se situent dans « un enracinement illusoire et un déracinement relatif » (Weismann 2012 : 50). Fascinée par une langue où « le mot évoqué s'inscrit dans une sorte de faille voire de distance » (Weismann 2012 : I4), Mali égrène une suite de mots qui, tout en relevant d'une même racine, esquissent des sens différents, à savoir Janna qui désigne le paradis. Provenant « de la racine trilitère Janna qui, voulant dire obscur, voilé, caché, nocturne, a aussi donné naissance à Janane, l'âme, le cœur [...] à Janine le fœtus, à Jounna les gens [...] à Jinni, les démons, les djinns [...] à Jinniya, la fée, la sirène, la sorcière, à Jounoune, la folie, l'égarement [...] la passion à majnoun, le fou, le dément $[. .$.$] ». Dans une langue où l'intelligibilité naît$ de l'altération, l'identité souveraine ou la racine qui est à la base du mot « accueille en son sein l'altérité rebelle, en lui permettant de s’affirmer sous forme de plusieurs identités relatives » (Weismann 2012 : 167). Promouvant en quelque sorte une conscience cosmique, la langue arabe « use d'une racine commune pour la terre et pour l'homme : l'œil en arabe, al'ain c'est également la source d'eau et c'est l'essence ontologique, c'est enfin ou d'abord la lettre de l'alphabet, celle que l'on emploie en dialectal -yáain- pour dire « que c'est beau », « ceci est un rêve » (Eddé 2003 : 58).

Hantée tout autant que Farid par la question des langues, Mali semble avoir appris de par sa fréquentation intime avec la langue arabe, laquelle aménage en son sein « l'espace de la réflexivité » (Weismann 2012:8), à se tenir à égale distance de la certitude comme de la perplexité. Initiée par cette langue où dès le « départ ne se trouve pas l'identité mais une séparation », Mali parvient finalement à résoudre ce faux dilemme qui l'acculait à penser une langue contre l'autre.

Dans leurs ouvrages Dominique Eddé, Salah Stétié et Amin Maalouf, choisissent la langue de l'autre pour évoquer un passé millénaire de rapports plurilingues et interculturels. En véritables magiciens de « l'interculturel », ils semblent promouvoir moins une nécessité de croiser les traditions voire de les hybrider qu'une volonté dans leur rapport avec leur identité, leur culture et même avec leur langue de s'installer dans la perplexité. Or seuls les initiés sont capables de trouver dans le principe de contradiction immanent à l'identité, à la culture comme à la langue cette espèce de dimension autre qui échappe à celui à qui « les choses se présentent avec une allure de valeur incontournable » (Weismann $2012: 34$ ). Marqués, à 
l'instar de Mali, par rapport à l'identité, Stétié et Eddé semblent proposer tant à l'Occident quà l'Orient le même pacte herméneutique, à savoir celui de la pensée dédoublée : vu que ce sont de part et d'autre des représentations, demeure par conséquent « le petit doute, la question : Est-ce que c'est vraiment ça ? » (Weismann 2012:34). C'est une fois cette question posée, qu'advient à l'existence ce « cœur intelligent 》 (En allusion au titre de l'ouvrage d'Alain FinkielKraut, Un cour intelligent) qui, du fait qu'on habite autrement sa langue et un ailleurs, permet de capter dans la fulgurance d'une transmutation alchimique le Même et l'Autre mais sous un jour inouï, à l'image un peu de Rome en qui Mali perçoit « un Orient concrétisé par [un] Occident » (Dominique Eddé 2003 : 195). C'est seulement alors qu’on peut reprendre l'expression de Georges Balandier « Civilisés, dit-on » mais, cette fois, à la forme affirmative.

\section{Références}

Badie Bertrand, 1996, «L'Occident et le monde musulman », in Badie Bertrand \& Sadoun Marc (Dirs), L'Autre. Études réunies pour Alfred Grosser, Paris, Presses de la Fondation nationale des sciences politiques, 237-250.

Brun Jean, 1985, L'Homme et le langage, Paris, Presses Universitaires de France.

Eddé Dominique, 2003, Cerf-volant, Paris, Ed. L'Arpenteur.

Kaufman Jean-Claude, 2oro, Linvention de soi, Paris, Fayard/Pluriel.

Maalouf Amin, 1999, Les identités meurtrières, Paris, Grasset.

Maalouf Amin, 2009, Le dérèglement du monde, Paris, Grasset.

Shayegan Daryush, 1992, Les Illusions de l'Identité, Paris, Ed. du Félin.

Shayegan Daryush, 2008, La Lumière vient de l'Occident, Paris, Ed. de l'aube (Diffusion Seuil).

Shayegan Daryush, 2012, La Conscience métisse, Paris, Albin Michel (Bibliothèque Idées).

Stétié Salah, 2008, Ouvrage collectif, « Le Liban au cœur du français », in Regards sur les littératures francophones du Moyen-Orient, Egypte, Liban, Interculturel, Francophonies, 14, nov.-déc., 16-33.

Verbunt Gilles, 20II, Penser et vivre l'interculturel, Lyon, Ed. de la chronique sociale. Weismann Heinz, 20I2, Penser entre les langues, Paris, Albin Michel (Bibliothèque Idées). 



\section{Decentring France: Multilingualism and the French language in Philippe Lioret's Welcome (2009)}

\section{Introduction}

Treating multilingualism as both a dialogic and narrative focus, contemporary French multilingual cinema re-envisions the place of multilingualism in French society, interrogating the complex power dynamics at play in multilingual interaction. One such film which places the dynamics of language and power at its heart is Philippe Lioret's 2009 Welcome, a film which calls into question the shifting role of multilingualism in France, and its impact on the role of the French language. Comparing Welcome's radical remapping of France and the migrant trajectory to earlier representations of French immigration in films such as Le Thé au harem d'Archiméde (Mehdi Charef, 1985), La Haine (Mathieu Kassovitz, 1995) and especially L'Esquive (Abdellatif Kechiche, 2003), this chapter investigates how Welcome offers an alternative view of the migrant experience, casting its gaze across the English Channel and thus "decentring France", along with the French language. Central to our argument are issues of border crossing and the boundaries of the French nation, which serve to include and exclude Welcome's characters, both physically, through movement across borders (or entrapment within them) and symbolically, through language use. We will thus explore how Welcome's representation of "boundaries, space, and territory [shape] the dynamic of cultural negotiation that fuels the film" (Murray Levine 2008 : $46-47) .{ }^{1}$ This chapter focuses in particular on the role of multilingualism

I Murray Levine refers here to Malik Chibane's 1994 Hexagone, however her words are remarkably applicable to Welcome as well. 
in Welcome, exploring how the international impacts on the local in the context of contemporary French society, and how this dynamic is expressed through multiple language use.

A transnational film on both a narrative and production level, Welcome represents the contemporary French nation as a multilingual space. The film follows Simon, a newly divorced swimming instructor who is drawn into a complex relationship with a young Kurdish immigrant. Seventeen-year-old Bilal has walked thousands of kilometres from his native Kurdistan to rejoin his girlfriend, Mina, whose family has recently migrated to London. Stranded alone in the French port city of Calais, Bilal finds himself unable to hitch a ride through the tunnel, despite paying hundreds of euros to a people smuggler. In desperation, Bilal enlists Simon's help, to learn to swim the Channel. While the personal connection between Simon and Bilal develops, and as Simon's marriage irreparably disintegrates, the French immigration police close in on each of the protagonists, hunting down Bilal to sanction and deport him, and threatening Simon with legal action for sheltering and aiding an illegal immigrant, a crime in France.

Set mostly in Calais, Welcome portrays a hostile social landscape resistant to linguistic and cultural diversity, a dynamic which plays out as much in private spaces as in public ones. Despite this, the film portrays multilingualism as emblematic of a contemporary world defined by immigration and globalisation, to the dismay of the film's many xenophobic and protectionist French characters. Michaël Abecassis has claimed that "the issues of ethnicity and plurilingualism in a multicultural society are central to contemporary French cinema" (Abecassis 2010 : 34) and Welcome is a powerful example of such foregrounding of linguistic diversity in film. In Welcome, the politics and practical implications of globalisation are at the forefront of social interaction and therefore language use. Indeed, in the film it is not only knowledge of French which is relevant to intercultural communication and indeed survival, but English and even Eastern languages typically associated with social marginalisation, such as Kurdish. 


\section{Multilingualism in Welcome}

In its harsh portrayal of the migrant experience on French soil, Welcome represents the French nation as a site of conflict and crisis, as well as of cultural fluidity and linguistic exchange. The multiple languages which dominate the dialogue of the film (French, English, Kurdish and a few brief excerpts of Pashto) are symbolic in undermining the neatly defined "Frenchness" of Simon's home, Bilal's supposed position of cultural disenfranchisement, the French characters' experience of their home nation and understandings of national territory and identity. As a result, Welcome posits even the most private of "French" places as a transnational, political and multilingual space. In this space, the once-monopolistic place of the French language is called into question. As Paul Bandia explains in his analysis of multilingual cinema, Welcome undermines the very "Frenchness" of French film:

As a result of the co-existence of these languages, the multilingual text is located inbetween languages, never entirely settled in one or the other language, constituting what one might call a hybridized text in a perpetual state of translation. (Bandia 2008:168)

It is significant that Simon and Bilal's relationship, and their respective interest to the Calais immigration police, subsequently becomes the central focus of the film's narrative, in which language is a key element. Through this relationship,

Welcome examines the negotiation of linguistic territories and suggests that [the film offers] a challenge to conventional assumptions about linguistic assimilation and, through language, an alternative to the fraught assumptions of migrant relationships as those between host and guest. (Smith 2012: 76)

Indeed, Simon's literal role as host to Bilal in his own home brings Smith's host-guest dynamic, depicted more broadly in the film's portrayal of France as (unwilling) host and immigrant as (undesired) guest, sharply into focus. As we shall see, the multiple transnational forces pulling at the fabric of the once closed-minded Simon's quintessentially "French" world are simultaneously embodied in the film's representation of place and language. 
From a promotion and reception studies standpoint, it can be extremely interesting to observe one of the main trailers for the film, for its portrayal of Welcome as a fundamentally, if paradoxically, French and yet multicultural film. The trailer, released in both Francophone and Anglophone countries, opens with an image of a ship leaving the Calais port for England. Bilal is shown negotiating ways in which to leave France, struggling to secure shady, overpriced deals with smugglers, staring longingly across the grey expanse of water that separates him from England, and ultimately training to swim the Channel. There is talk of Iraq, of Kurdistan, of London. Simon's apartment is shown being penetrated by xenophobic neighbours and suspicious immigration police. The events portrayed are at once personal and political. And the final image is of Bilal in the water itself, desperate to reach English shores. Accompanying all these images is dialogue in three languages, and both of the film's protagonists are shown speaking in more than one language each (Simon in French and English, Bilal in Kurdish and English), all in the space of a minute and a half.

In a rare and noteworthy move, this trailer presents Welcome from the outset as a fundamentally multilingual product, marking the film's language diversity as a key element of its promotion. Yet it does so while also promoting the film as "French". Thus, despite the superimposed text, accompanying a number of festival award logos, proclaiming Welcome as "the most celebrated French film of the year", the simplicity of the film's French label is persistently called into question in this brief clip. The film can be reasonably considered as French, due to its funding, production and filming location, and the nationalities of a large proportion of its cast and crew, but it is also proudly international, multicultural, transnational and multilingual. Indeed, Welcome's dominant language is, unsurprisingly, French, but also, in almost equal measure, English. Through this foregrounding of English language use, the film's position remains clear: in stark contrast to cinéma beur films of the late twentieth century, in which representations of immigration paint France as the ultimate destination and French as the ultimate linguistic prize, Welcome's geographical, cultural and linguistic focus is elsewhere.

In fact, far from a passing or background language, English is central to Welcome. English becomes a lingua franca in the film not because of its political or social connotations, but because it is the linguistic common 
ground between two nationalities which do not share any direct historic links. The traditional immigration narrative in French film generally features a combination of protagonists from previous French colonies (namely North Africa), as well as a number of French characters. Thus French is the shared language and the lingua franca used. However, in Welcome, the immigrant in question has not found himself in France because it is the logical destination to take refuge in, due to colonial heritage and thus shared language. Born in Kurdistan, Bilal is fleeing the recent (and at the time of the film's creation, current) Iraq war and is merely passing through France, in the hope of reaching his final destination: London. Thus English becomes the primary language of use in a location in which the lingua franca would traditionally be expected to be French.

In Welcome, English therefore becomes what Chris Wahl describes as "a common ground and a strange world" (Wahl 2005 : 3). English is a secondary language for all of the film's characters, and is spoken in only one scene on Anglophone soil. And yet English is the only language understood by both the protagonists, and by almost every character in the film (including Mina and Simon's [ex-] wife, Marion). English is "a strange world" in its disconnectedness from the characters' cultural identities and origins, but a powerful "common ground" in its function as a shared means of communication. Indeed, English functions as a lingua franca and a useful egalitarian code where Kurdish and even French (indeed, any other language at any of the characters' disposal) cannot:

For Lioret, English is a displaced language, with only the most tentative connections to territory, and it is on this condition that Bilal and Simon can use it to step out of their respective linguistic houses. (Smith 2012 : 79)

Thus English offers Simon and Bilal the opportunity to build a relationship despite the many fundamental differences and barriers which divide them. Alison Smith proposes the label "threshold language" (Smith 2012) to describe this use of a mutual second language; for both Simon and Bilal, English is a foreign yet comprehensible language, which allows them to interact meaningfully despite their myriad cultural and social differences. French does not offer this possibility; it is Simon's native language, the language of the authorities which threaten Bilal, and a language the latter 
does not speak. English offers the only possibility for Simon and Bilal to communicate on an equal linguistic footing.

Indeed, almost unprecedentedly for a French film, the most linguistically competent character in Welcome is not the white native French-speaker Simon, but the adolescent Kurdish clandestine immigrant, Bilal. Bilal's English is almost perfect where Simon's is certainly competent, but more faltering. In conversations with other Kurdish characters, Bilal acts as an interpreter for Simon and other French characters. In fact, Bilal is the only character who actually acquires new language in the film: while he only manages a rudimentary grasp of French, towards the end of Welcome Bilal tries to communicate with Simon in his native language.

The positioning of trilingual Bilal as the character best adapted to his globalised environment evokes the work of Hamid Naficy, who has coined the term "accented cinema" to describe the contemporary phenomenon of films in which multilinguality "feeds into and feeds off the horizontality of our globalized world, where compatriot diasporic communities are in touch with each other laterally across the globe, instead of being focused on an exclusive binary and vertical exilic relationship between the former home country and the current homeland" (Naficy 2010: 15). In line with Naficy's argument, Bilal's linguistic openness and flexibility thus posit him as the film's most progressive and best-adapted character; not merely its youngest, but also its most at home in the globalised, transnational universe that Simon initially tries to ignore, despite it having arrived at his very doorstep.

Both Bilal's linguistic aptitude and the importance of English in the film can be observed in the scene in which Simon first invites Bilal into his home. One evening, Simon sees Bilal and his Kurdish friend Zoran wandering the streets and calls out to them in English: "where are you going? Come". Zoran, who is a stranger to Simon and has a very limited understanding of English, hesitates. Bilal, who knows and understands Simon, turns to his friend and speaks in Kurdish. The two then approach Simon's car. Bilal's Kurdish speech is not subtitled in this scene, and therefore it is unclear to a non-Kurdish-speaking viewer whether his words contain an explanation of Simon's trustworthiness, but it is clear that a translation is taking place for his practically monolingual friend.

In Simon's apartment, as the three men enjoy a meal of beer and pizza, Bilal is called upon to act as interpreter. At one point, Simon asks Zoran, 
in English, how he plans to find a job in England. Zoran catches the word "job" and responds "a job? Yes", then turns to Bilal and speaks in (unsubtitled) Kurdish. Bilal translates for Simon (and the non-Kurdish-speaking audience): "his brother has a job at a supermarket in London. He can get a job for him too". Unable to communicate beyond a couple of words in either English or French, Zoran is certainly at a linguistic disadvantage, yet can rely on Bilal's English skills to translate for him. Simon, however, has no such translator for his French-language dialogue. At a number of emotional moments during Bilal and Zoran's stay, Simon lapses into French, despite neither guest understanding him. Indeed, at one point Simon shouts at Zoran, in rapid-fire French, to get away from him, with no attempt at translation into English, for Bilal to translate in turn into Kurdish. Zoran reacts heatedly in Kurdish, yet due to the lack of bridging English-language dialogue, Bilal is unable to mediate the men's altercation. It is one of the film's most hostile moments, and one of the only situations in which the characters fail entirely to communicate meaningfully with each other.

These scenes include the most diverse range of linguistic backgrounds to be found in the film: a monolingual Kurdish speaker, a bilingual Kurdish and English speaker and a bilingual English and French speaker. Between Bilal and Zoran, Kurdish is a familiar and shared language. Between Bilal and Simon, English is a functional, if mutually secondary, means of communication. Simon, however, is alone in his understanding of French. As a result, despite these scenes being primarily set in Simon's private Calais home, and despite the limited cultural capital of a marginalised language such as Kurdish in French society, French is the least useful, and indeed the most destructive, language at play. If Simon were not also conversant in English, he would not be able to communicate with either of his guests. And Bilal, with his fluency in both Kurdish and English, is the only character capable of fully navigating the complex, multicultural situation in which the three characters find themselves. ${ }^{2}$ Philippe Lioret, 2009. Actors: Vincent Lindon (Simon), Firat Ayverdi (Bilal), Selim Akgul (Zoran). 
Previous transnational French cinema, from cinéma beur to the postcolonial cinema of filmmakers such as Claire Denis, has engaged on many levels with issues of multiculturalism: cultural isolation, social fracture, racism, integration, exile, intergenerational conflict, delinquency, violence, unemployment etc. At the heart of many transnational films lies a fundamental interest in issues of culture and power. Patricia Caillé captures this interest in her article "'Cinemas of the Maghreb': Reflections on the Transnational and Polycentric Dimensions of Regional Cinema”:

The transnational enables us to interrogate relationships of power and domination
in a digital and post-industrial age, at a time when the accrued circulation of cultural
commodities and people (either forced or voluntary) affects the terms of the polarity
between nation and migration. (Caillé $2013: 242$ )

However, throughout the twentieth century these issues were largely explored monolingually. Language is depicted as a differentiating factor, a means of identification and especially as a system of belonging in films like La Haine, but generally only in French: cultural and social fracture is explored not through multilingualism, but through linguistic variations within French, such as verlan and argot. If foreign languages are included in the dialogue, it is often in the background (rendered devoid of semantic meaning through a lack of subtitles) or passing words or phrases (which Chris Wahl terms "postcarding" [Wahl 2005: I] giving a film a multilingual "flavour" without exploring multilingualism in any real depth). Welcome sits in stark contrast to these earlier films, in which language diversity plays a background role. For in Welcome, many of the key scenes in which characters find themselves in various states of social and cultural conflict take place in and around language: through interpreting, code switching and language learning.

In fact, it is significant that Simon's (ex-) wife Marion, the film's most culturally open-minded French character, is a primary school English teacher. Marion also uses English nightly in her interaction with the immigrants camping near the docks, as she runs a soup kitchen. While she is hesitant to support Simon's sheltering of Bilal and his friend Zoran, as she knows Simon could be arrested for doing so, Marion is constantly posited as the most "welcoming" of all Welcome's characters. She not only feeds 
Calais' immigrant population, but stands up for them when they are persecuted, as when they are refused entry to the local supermarket she and Simon are shopping in. There is every suggestion that Simon, desperate to win Marion back, even embarks on his relationship with Bilal as a means to impress her. While given comparatively little screen time, Marion is a catalyst for many of the film's events, and is often posited as its moral compass. It is thus significant that she is so clearly associated with foreign (specifically English) language use throughout the film.

Additionally, the film's key resolution scene also takes place in Smith's "threshold language"; following Bilal's drowning, the Kurdish Mina and the French Simon meet on foreign soil (London) and communicate in a second language (English). Despite each being displaced from their home, not speaking in their mother tongue and being complete strangers to one another, Mina and Simon can begin to resolve some of the film's most painful details. Mina is assured of Bilal's devotion to her and informed of his plans to swim the Channel to reach her and his ultimate failure to do so. Simon is able to share his loss with the only other person he knows of who feels deeply for Bilal. And the film's recurrent motif of the wedding ring, constantly lost, rejected and refused, finally finds its home in Mina, despite the impossibility of its purpose being realised. While steeped in grief, this scene between Simon and Mina is the film's sole moment of closure for either of the two characters. In this crucial moment, French has no place, and indeed no power to soothe either Mina or Simon. Unalike in almost every sense, the two characters' common knowledge of English is essential to their ability to achieve this closure at all.

\section{Welcome's foregrounding of place and movement}

Welcome's mise en scène is dominated by a number of loaded and symbolic settings (the swimming pool, the beach, the soup kitchen, the refugee shanties which the French characters refer to hauntingly as la jungle) and 
not least the supposedly private space of Simon's apartment, where he lives alone following his marriage breakdown. The apartment is the site of many of the film's key scenes of conflict, confrontation and change. These range from Simon's initial bonding with Bilal to violent confrontation when Zoran steals Simon's coveted Olympic medal. From arguments with Simon's xenophobic neighbour, the owner of the terribly ironic "Welcome" mat from which the film takes its name, to heated altercations with the police during their unsolicited visit in search of Bilal. As the film progresses, the conflicts and character evolutions that take place within the confines of Simon's apartment draw him further away from an insular private life, and open him up to encountering a multitude of individuals, world views, cultures and languages. In other words, mimicking the film's trajectory more broadly, the local site of the French home becomes increasingly defined by the global, the foreign and the unknown.

Likewise, the broader location of the city of Calais is symbolic. Much like the Western French port city settings of such comparable contemporary French films as Le Havre (Aki Kaurismäki, 20ıI, set in Le Havre) or Mapart du gâteau (Cédric Klapisch, 20II, set in Dunkerque), the space in which Welcome's story unfolds is perched on the perimeter of the French territory. Situated on the shores of the English Channel, equipped with ports, ferries and an entry point into the subterranean tunnel to England, Calais is in essence a place of passage, migration and flow, particularly the flow of peoples:

It is as well to remember that [this] linguistic landscape [is] negotiated, and negotiated, almost certainly, to play on deeply anchored cross-Channel rivalries, as well as observations on migrant flows. (Smith 2012:87)

Indeed, it could be said that the entire film revolves around characters in a state of flux or transition. Bilal and his fellow migrants are in a constant state of migration, homelessness, border-crossing and uprootedness. On a more symbolic level, Simon and Marion also find themselves in an in-between state of relationship breakdown; moving house, splitting belongings and finalising their divorce. Even Simon's job, which leads Bilal to approach 
him in the first place, is centred on movement, through the act of swimming (although it is Bilal who transforms the stagnant repetitiveness of lap swimming [a movement which ultimately goes nowhere] into a means of physical border-crossing, of transport).

Thus Welcome is characterised by movement, but also by the inability to move. Just like the paperless protagonist of Rabah Ameur-Zaïmeche's 2001 Wesh-wesh, qu'est-ce qui se passe? Kamel, the French nation and its rigid immigration policy serve to trap Bilal within its borders. Murray Levine says of Wesh-wesh, in a description equally applicable to Welcome:

These visual elements reinforce the prison-like character that the cité holds for Kamel, who cannot work or circulate freely because of his illegal status. (Murray Levine 2008: 50)

This is one of the key aspects which differentiate Welcome from so many other films, contemporary or otherwise, which depict immigration; France is not the desired final destination. France has not been chosen as the immigration destination for its shared language, historical familiarity or colonial ties with the country of origin of the migrant. In fact, Bilal doesn't want to be in France at all. France is merely one of the many countries he passes through on his way to England; the only thing that differentiates it from these other countries is that, because of the Channel, he is stuck there. Welcome belongs to a group of contemporary films which explore this border-crossing conundrum, which Alison Smith describes as "the interest in representing on film that part of France, especially the margins of France - the ... coast and the outskirts of the large cities - that is increasingly a cultural contact zone" (Smith $2012: 76$ ).

In so many French films about the migrant experience, making it to France is the objective of the migrant (although, of course, upon their arrival, the glowing ideal of France is generally deconstructed). In Welcome, the opposite is the case: France serves only to delay and jeopardise Bilal's migration. This revises the geographical and symbolic mapping of the migrant trajectory, shifting the migrant's focus elsewhere, decentring France and situating it within the context of a broader, transnational setting. 


\section{Remapping the migrant trajectory}

In light of this (re)mapping, the city in which Welcome takes place is again significant. While London leaves its mark on the film in a number of ways (such as linguistic influence, the scenes filmed there and Bilal's desperation to reach it), Paris is completely absent from Welcome. Instead, the film is literally situated in an exit point from the country. In this way, Calais is representative of the role of France itself in the film: a halfway point, a transcultural space, a place of transit rather than a destination, representative of movement and uprootedness. Calais is shown to be what Marc Augé sees as a "non-lieu": a "non-place", or at least an in-between space devoid of identity (Augé 1992). The very nature of Calais as a place of transit and impermanence mirrors the plight of the immigrants trapped there, and of the role of France in Bilal's journey. This paints the migrant experience in a radically different way to the majority of preceding French films about immigration. Let us turn now to an examination of these earlier films in order to understand the extent to which Welcome remaps the migrant trajectory in France, and revises the role of the French language in the migrant experience.

In the vast majority of French films which explore immigration, France is typically oriented as the migrant's focus, as the locus of Western civilisation, the centre of the civilised world. Even when characters undertake pilgrimages or cultural voyages, such as in Tony Gatlif's Exils (2004), they begin their journey from Paris, and usually return to it. No matter how negatively France may be depicted in any of these films, and no matter how disillusioned the migrant characters may become about their host country, it is significant that France and especially Paris are still mapped as centre. Upon arrival, the migrants in these films, and their second-generation children, almost always face a harsh and disillusioning reality. Yet these first-generation characters have nonetheless fought to make it to France; they have not settled in France by chance, by force or as a last resort. France is the object of their migration and their struggles to found a new life. This mapping reinforces Alec Hargreaves and Leslie Kealhofer's claim that traditional French films about immigration underline how the 
"protagonists are rooted in the dominant language and cultural norms of France" (Hargreaves and Kealhofer 20I0, pp. 75-76), no matter their struggles with daily life and integration.

A particularly prominent example of a North African protagonist's desire to reach or even idolisation of France is Abdellatif Kechiche's $200 \mathrm{I}$ La Faute à Ronsard, which revolves around the protagonist Jallel's desire to get to France, especially Paris, and to stay there. Jallel pushes a halfFrench friend into agreeing to marry him for citizenship, and is thrown into a deep depression when the arrangement falls through. Throughout the film, despite his Tunisian origins and his lowly social status in France, Jallel chooses to surround himself with what he considers to be hallmarks of French culture (literature, music, art), even attempting to make a livelihood from them, performing Voltaire poems in the metro. He cultivates a relationship (albeit a dysfunctional one) with a French girl. And the film's ultimate tragedy is Jallel's capture by French immigration police, signalling his inevitable deportation.

Likewise, films like Inch'Allah Dimanche (Yamina Benguigui 2001), Code inconnu: récits incomplets de divers voyages (Michael Haneke 2001) and Wesh-wesh, quest-ce qui se passe? are filled with characters originating from Northern African or Eastern European countries, who have endured all manner of physical, social and cultural upheavals to make their way to France and attempt to build a life and a family in what is invariably presented to them as a land of opportunity. Even in so recent a film as Costa-Gavras' 2008 Eden à l'ouest, the protagonist Elias risks his life on multiple occasions to reach France which, as the title suggests, he imagines is a sort of paradise on earth. In these films, the ideal of France is invariably unravelled upon the characters' arrival there. Nonetheless, France is almost always presented to these characters as the focus of their migratory movements and the ultimate destination in which to settle and prosper.

Subsequently, the French language's place in this mapping of the migrant trajectory is a dominant one. These films constantly underline the essentiality of knowing French, positing the migrant's learning of French as a rite of passage on the path to successful cultural integration. They also show the inevitable isolation and disadvantage of not learning French, a common fate for many first-generation women, who are so often confined 
to the domestic sphere and cut off from French civilisation (Inch'Allah Dimanche). The differences between first-generation parents, comparatively marginalised and disempowered in French society, and their more integrated children, are often crystallised in the latter's superior knowledge of French, and related conflicts brought to a head through their rejection of their parents' native language in favour of their birth country's tongue. As Carrie Tarr explains, in films such as "Le Thé au harem d'Archimède language is a source of conflict and misunderstanding" (Tarr 2005: 56), placing French in the clear position of a more socially and cultural valuable language.

In these films, which are almost always set in the banlieue (the low socio-economic housing commission estates on the outskirts of the city), Paris is invariably mapped as centre. The narrative and cinematographic make-up of many such films constructs the relationship between the banlieue periphery and the metropolitan centre as an evocation of inner and outer, belonging and not belonging, French and Other. In this environment, the dividing line of the périphérique, once a wall and now a bustling highway, is symbolic as a physical and cultural barrier between the city and the banlieue, positioning the inhabitants of the banlieue as unintegrated, not belonging, excluded. Most films de banlieue construct this binary not merely through cinematographically representing the banlieue as an external space in contrast to Paris, but in showing its characters' awareness of this space. These films do not locate themselves solely in the environment of the banlieue, but their banlieue-inhabiting protagonists physically travel into the city's nucleus, where their outsider status is literally, and often violently, confirmed.

Perhaps the most prominent example of this is Mathieu Kassovitz's 1995 La Haine. In the film, the three main characters Vinz, Saïd and Hubert travel by RER train into central Paris, where they find themselves alienated by their bourgeois surrounds, including a 7 th arrondissement apartment and a pretentious gallery event. While the protagonists are certainly free to enter the city, they are consistently made to feel as though they do not belong. This confirms their othering even more than their exile in the far reaches of the city's commission flats. And in failing to jack a car and in missing the last RER, they are effectively trapped in the city overnight. They 
can enter, but cannot leave (and cannot rest, confined to the hostile Paris streets). Yet despite the hostility of the city centre, Paris remains an ideal, epitomised in the image of the three men looking out over the cityscape at the silhouette of the Eiffel Tower, as well as the billboard posted along the train line promising that "Le Monde est à vous" ("the world is yours").

\section{A key point of comparison: L'Esquive}

No discussion of the evolution of the migrant trajectory in French film, moving from the binary dynamic of beur and banlieue cinema to the radical decentring of France which occurs in Welcome, would be complete without acknowledging the progress made in L'Esquive. Like Le Thé au harem d'Archiméde and La Haine, L'Esquive is a film which has found itself categorised as a film de banlieue: the film is situated in one of the notorious commission flats in Paris' north-east region of Seine St Denis, the film deals with some typical issues characteristic of banlieue narratives (delinquency, multiculturalism, police aggression) and the dialogue is deeply marked by youthful banlieue-centric language (for example, use of the reversed-French code verlan, or the Arabic-infused variation of French known as tchatche).

Yet L'Esquive differentiates itself from Le Thé and La Haine's constructions of the banlieue/city centre binary in fundamental ways. For one, the characters never in fact leave the confines of the banlieue, and many of the scenes are shot in outdoor surrounds, often in amphitheatres or parks rather than underpasses or car parks, avoiding the aura of oppression or claustrophobia which so characterise the mise en scene of traditional banlieue films. Likewise, there is no talk of the city or indeed the geographical reality beyond the banlieue; despite being only a few kilometres from Paris, the banlieue, not the city, becomes centre. This sets the mise en scene of the film de banlieue up for a radical update: as Vinay Swamy remarks, "Not all banlieue films seek to highlight these issues within an explicitly violent framework that supposedly characterizes the tension between the centre and the periphery" (Swamy 2007:58) 
There is still some travelling done in L'Esquive, yet it is Marivaux, not the film's characters, who travels between the banlieue and the city. A traditional and culturally validated motif of "French-ness", a symbol of French cultural hegemony, Marivaux is a sign of the mainstream French symbolic past, of a time before the banlieue and the cultural melting pot of contemporary France, associated with a language completely removed from the arena of verlan, tchatche, indeed even standard French. Marivaux's I730 play Le jeu de l'amour et du hasard permeates the film, as the characters prepare a performance of the play at their school. Not only do the characters accept and even embrace this symbol of Frenchness, they do so on their own turf. Therefore, while Marivaux is a key element of the film (and the play a mise en abime of the story) Paris is not. The way in which L'Esquive thus understands and presents urban and suburban space foreshadows Welcome, and reflects what Alison Murray Levine writes about film space and borders:

An aesthetic and thematic shift [is taking place] in recent beur cinema toward the portrayal of borders as permeable boundaries and of formerly excluded spaces such as the banlieues as sites of dynamic cultural exchange. (Murray Levine 2008:43)

While there is no explicit Arabic or other foreign languages spoken in the film, it is important that several language variations coexist within L'Esquive: the elevated and antiquated French of Marivaux's play, the standard, "correct" French of classroom discussion and the street French, laced with verlan and tchatche, spoken among the young characters. Verlan and similar slang registers are present in many films, from La Haine to Laurent Cantet's Entre les murs (2008), yet while such films certainly acknowledge the widespread use and significance of these codes, they often also focus on their social inferiority. For example, in Entre les murs, the teacher Monsieur Marin forbids the use of verlan in the classroom, saying he will only communicate with people speaking "French": "en français, s'il te plaît", denying verlan's place in any part of the French language at all.

Yet street language is not imbued with the same connotations of linguistic hierarchy in L'Esquive; there is no moment when a standard French-speaking character criticises or forbids such language use. Indeed, each register of language used in L'Esquive is treated (by the film and the 
characters) as equally relevant and valid in its own context. Vinay Swamy suggests that L'Esquive "sets itself apart in the way it reframes the relationship between high and popular culture through its explicit and very conscious use of language" (Swamy 2007 : 58), a relationship entirely absent from films like La Haine, in which slang only serves to disconnect and disenfranchise the banlieue-dwelling characters from French society.

In fact, while language and power are crucial themes in L'Esquive, the linguistic power in the film lies not in the mastery of a culturally validated language such as Marivaux's French, or even "correct" standard French, but in the ability to move convincingly between the numerous cultural spheres and their respective language uses with verve. For example, the protagonist Krimo is almost completely incompetent at switching between language forms. On the other hand, the film's female lead, Lydia is somewhat of an arch linguist in her ability to master the three registers involved, moving effortlessly between them and employing them in the appropriate circumstances. As Dana Strand writes,

[Lydia embodies] the fluid passage among linguistic registers so effectively carried out by at least some of the young people in L'Esquive ... the cast of unlikely characters glides smoothly from the rarefied linguistic expression of eighteenth-century high culture to their graphically gritty slang, thus calling into question the historically sacrosanct place accorded to the French language in the construction of national identity (Strand 2009: 264-265)

Not only does Lydia find the language of Marivaux natural to slip into, but she is also capable of switching immediately into street language to communicate with her friends. Lydia is in fact French, but employs Arabic-infused phrases such as "Inch'Allah", where other characters do not, to assert the fact of her belonging to the multicultural banlieue community. Indeed, the film's ultimate image is of Lydia performing on stage, her voice the centre of attention, while Krimo is exiled, on the other side of the theatre window, silently (and deafly) watching the performance play out in the distance. Here we clearly see an inner/outer binary, reminiscent of La Haine and its cinéma de banlieue predecessors, and it can of course not be ignored that Lydia is a French character performing in Marivaux French and Krimo a second-generation North African immigrant unable to express himself in 
the required language. Yet it is likewise extremely significant that the entire storyline plays out in the banlieue alone.

L'Esquive takes an important step in the direction of re-orienting the immigrant experience and the significance of France, and especially Paris, as centre, and ground zero for migration. Claudia Esposito echoes this in her article "Ronsard in the metro: Abdellatif Kechiche and the poetics of space” (20II), emphasising L'Esquive's unique representation of (sub) urban space:

Kechiche's films expand and enrich a debate around contemporary French national identity that takes shape, as several critics have demonstrated, around questions of language and space. (Esposito 2011 : 224)

\section{Welcome: Conclusions}

L'Esquive's understanding of the migrant's cultural and linguistic experience provides a stark counterpoint to traditional, Eurocentric representations of the migrant trajectory. However, Welcome takes this re-orientation, indeed decentring, vastly further. Leaving Paris and its surrounds behind, perching its story on a literal exit point from France to the UK and infusing the film's dialogue with an array of foreign languages, Welcome radically turns away from the French cultural nucleus, re-envisioning the traditional migrant journey and positing London, rather than Paris, as the migrant's desired destination. As Dale Hudson writes:

Transpolitical spaces within transnational French cinemas offer opportunities for investigating the inequalities within this nationalist bundling of people, territory and politics, particularly around borders (Hudson 2011 : 113).

And Welcome places this notion at its core. Welcome belongs to a collection of films which interest scholars such as Carrie Tarr for "their representation of spatial and bodily border crossings" which in turn "expose the asymmetrical power relations between hosts and migrants, Western 
Europe and its others" (Tarr $2007: 7$ ). Yet France's place in this relationship "between host and migrants, Western Europe and its others" is far from the monopolistic, dominant one of the twentieth-century immigration films explored in this chapter. France is but one nation among many participating in the complex, polycentric flow of peoples taking place in Welcome's contemporary world.

This decentring of France in Bilal's migrant trajectory also serves to decentre the French language. French is certainly an important language in Welcome, especially in conversations between Simon and other French characters (Marion, his co-workers, his neighbour and the police). French, however, is not the main language at play in any of the important scenes between French and migrant in the film; English is. In Welcome, it is not French which is key to Simon's relationship with Bilal, to the events of Bilal's journey across the Channel, nor to the everyday experience of the majority of clandestine immigrants represented in the film, who have originated from far beyond France and all wish to travel to England. Instead, while French proliferates throughout conversations between French characters in the film, it is English which dominates Welcome in relation to Bilal and Simon's experience, the principal storyline and the film's narrative and thematic focus. Likewise, in Bilal's interaction with his fellow immigrants, and in his initial quest to reach English shores through smuggling, Kurdish and Pashto are of greater value to his negotiations than French.

The scope of the film thus extends beyond the centrality of France and situates it in a cosmopolitan and transnational world in which the reach of foreign nations not only impacts on the local Calais population, contributes to the Calais economy and geographically surrounds the city itself, but permeates the very fabric of the entire film: multicultural characters, constant talk of other countries (England, Iraq and Turkey), physical movement within and across borders and especially multilingual dialogue.

A film which engages deeply with contemporary issues of language, immigration and globalisation, Welcome represents its Calais environment as a site of cultural and linguistic diversity. In this environment, the integrity of the contained nation of France is eroded. The multiple languages which dominate the film's dialogue (French, English, Kurdish and Pashto) are symbolic in questioning the "Frenchness" of its Calais location, positing 
it in a transnational, political and multilingual space. In these shifting and disintegrating spaces, the supremacy and dominance of the French language, both as language of the host country and prerequisite of the immigrant, is fundamentally undermined.

\section{References}

Abecassis, Michaël, 2oro, "The Voices of Pre-War French Cinema: From Polyphony Towards Plurilingualism" in Berger Verena and Komori Miya (eds) Polyglot Cinema: Migration and Transcultural Narration in France, Italy, Portugal and Spain, Vienna, LIT Verlag, 33-48.

Augé, Marc, 1992, Non-lieux: introduction à une anthropologie de la surmodernité, Paris, Seuil.

Bandia, Paul, 2008, "Review: Fictionalising Translation and Multilingualism", in Target, 20-I, 164-169.

Caillé, Patricia, 2013, "Cinemas of the Maghreb': Reflections on the Transnational and Polycentric Dimensions of Regional Cinema", in Studies in French Cinema, I3-3, 24I-256.

Esposito, Claudia, 20II, "Ronsard in the Metro: Abdellatif Kechiche and the Poetics of Space", in Studies in French Cinema, II/3, 223-234.

Hudson, Dale, 20II, "Transpolitical Spaces in Transnational French Cinemas: Vampires and the Illusions of National Borders and Universal Citizenship", in French Cultural Studies, 22/2, III-I26.

Murray Levine, Alison J., 2008, "Mapping Beur Cinema in the New Millennium", in Journal of Film and Video, 3/4, 42-59.

Naficy, Hamid, 2010, "Multiplicity and Multiplexing in Today's Cinemas: Diasporic Cinema, Art Cinema, and Mainstream Cinema" in Journal of Media Practice, $\mathrm{II} / \mathrm{I}, \mathrm{II}-2 \mathrm{O}$.

Smith, Alison, 2012, "Crossing the Linguistic Threshold: Language, Hospitality and Linguistic Exchange in Philippe Lioret's Welcome and Rachid Bouchareb's London River", in Studies in French Cinema, I3/1, 75-90.

Strand, Dana, 2009, "Etre et parler: Being and speaking French in Abdellatif Kechiche's L'Esquive (2004) and Laurent Cantet's Entre les murs (2008)", in Studies in French Cinema, 9/3, 259-272. 
Swamy, Vinay, 2007, "Marivaux in the Suburbs: Reframing Language in Kechiche's L'Esquive (2003)", in Studies in French Cinema, 7/1, 57-68.

Tarr, Carrie, 2005, Reframing Difference: Beur and Banlieue Filmmaking in France, Manchester, Manchester University Press.

\section{Filmography}

Ameur-Zaïmeche, Rabah, 200I, Wesh-wesh, quest-ce qui se passe?

Benguigui, Yamina, 200I, Inch'Allah Dimanche.

Cantet, Laurent, 2008, Entre les murs.

Charef, Mehdi, 1985, Le Thé au harem d'Archimède.

Chibane, Malik, 1994, Hexagone.

Gatlif, Tony, 2004, Exils.

Gavras, Cost, 2008, Eden à l'ouest.

Haneke, Michael, 2001, Code inconnu: récits incomplets de divers voyages.

Kassovitz, Matthieu, 1995, La Haine.

Kaurismäki, Aki, 20ıı, Le Havre.

Kechiche, Abdellatif, 2001, La Faute à Voltaire.

Kechiche, Abdellatif, 2003, L'Esquive.

Klapisch, Cédric, 20II, Ma part du gâteau.

Lioret, Philippe, 2009, Welcome. 



\section{The Message of the Text and the Text of the Message in Two Contemporary French Films: LOL (Laughing Out Loud) and De Rouille et d'Os}

The fabric of texting is deeply embedded in the fabric of everyday life. Look around you: head-bowed, thumb-poised "textperts" are everywhere. They have been credited with developing a new shorthand language, vowel-poor, acronym-rich, and emoticon-laden that is largely unfathomable to older people. This new language has been praised by linguists for its creativity.

-ANTHONY PATTERSON

The popular impression, created largely by the media, is that the written language encountered on mobile phone screens is weird. It has been labelled "textese", "slanguage", a "new hi-tech lingo", a "hybrid shorthand", a "digital virus". It has been described as "foreign", "alien", and "outlandish". It is so much viewed as a new language that texters have been called "bilingual".

-DAVID CRYSTAL

Text messaging is prominently featured in two contemporary Frenchlanguage films: Lisa Azuelos's 2008 coming of age teenpic/romantic comedy LOL (Laughing Out Loud) ${ }^{\circledR}$, set in the world of upper class Parisian lycéens, ${ }^{1}$ and De Rouille et d'Os (2012), Jacques Audiard's haunting, critically acclaimed drama about loss, trauma and redemption. These two otherwise divergent narratives raise compelling questions about text

I Remade by Azuelos as the 2012 English-language LOL, starring Miley Cyrus in the titular role and Demi Moore as her mother. The remake is set in Chicago. 
messaging's creative and disruptive potential as a force for reinvention of the French language. The following discussion considers the linguistic as well as sociocultural impact/implications of the significant presence of texting in both films.

Text messaging, introduced in the 1990s, is popularly known as the "texto" or SMS in France (Anis 2007). According to Jacques Anis (2007) in his analysis of French SMS and neography, "the beginning of the millennium saw an explosion of mobile telephony in which SMS played a significant role. In 2003, 9.8 billion SMS messages were sent in France, including 88 million on New Year's Day alone". The globally popular phenomenon of text messaging raises important questions, especially for youth culture, since "by 2009, texting had become the most popular form of communication among teenagers, surpassing email, instant messaging, social networking, and face-to-face communication" (Patterson 2013: 84), as demonstrated by one of the two films discussed in this article, LOL (Laughing Out Loud) ${ }^{\circledR}$. $L O L$ 's eponymous protagonist and core group of high school friends are characters that exemplify the predominance of SMS use among adolescents. Texting has been particularly examined in conjunction with its relationship to/association with youth/adolescent culture (see for example the work of Crispin Thurlow): according to McKay et al., whose research considers "some of the many ways that technologies such as the internet and mobile telephones are influencing and influenced by teens' self-identity, relationships, social practices, family, schools, language and peer social groups" (2005: 186), “today's teens have been branded the 'net generation' or 'N-Gen"' (2005: 185) while Thurlow, a leading scholar of the sociolinguistics of the text message, dubs today's teens "Generation Txt" (2003) in an article of the same name. All of this is reflected in $L O L$, which explores the adolescent group dynamic of the film's eponymous leading character named Lol(a) and her friends as they experiment with the boundaries of friendship and love as well as explore their sexuality, frequently doing so via the language of new media, expressing themselves with abbreviations and neologisms as mediated through the technological apparatus of the laptop, and, most important for our purposes, the cell phone. While text messaging is not necessarily limited to teenagers, "the core mobile phone market is ... under-45" (Reid and Reid 2004: I) the age group to which the 
two protagonists of the other film discussed herewith, Audiard's awardwinning drama De Rouille et d'Os (2012), also belong.

Not only is texting "revolutionizing communication in today's society" (Reid and Reid 2004: I), but also, arguably, language itself, as it represents "a new language [which] has been praised by linguists for its creativity" (Patterson 2013: 84). Indeed, texting has been called "one of the most innovative linguistic phenomena of modern times" by David Crystal (2008: 172), and in a discussion of the inventive aspects of texting, its popularity among adolescents is especially worth noting, since "adolescents lead other age groups in linguistic change ... and quite probably in the coining of lexical items, discourse markers, intonation patterns, and so forth" (Eckert 2003: IIS). According to Cougnon and Beaufort in their work on SMS neologisms, "certaines caractéristiques de la pratique du sms entrainîent une très grande créativité linguistique ... l'écrit sms favorise la création ..." (20II: 19I). Thurlow and Poff, who explore the concept of a "particular 'language of texting"' (20II: I2) find that texting encapsulates the zeitgeist of our era, "where the diminutive, the brief, and the simple are highly prized in communication" (2011: 13); in Patterson's words, "a 'good' text message ... should be impulsive, flippant, and off-the-cuff” (2011: 87). ${ }^{2}$ Patterson further describes texting as "a new shorthand language, vowel-poor, acronym-rich, and emoticon-laden” (2011: 84), although he cites Carol Ann Duffy, Britain's poet laureate, as a proponent of the language of texting, since she "argues that its power, as a new genre of writing, lies in that fact that it 'allows feelings and ideas to travel big distances in a very condensed form"' (Duffy cited in Patterson 2013: 87). The emotional significance/impact of texting in both films - especially in regards to French-language texters - is discussed below.

Central to the diegetic unfolding of $L O L$ (Laughing Out Loud) ${ }^{\circledR}$ and De rouille et d'Os (2OI2), is how "the practice of text messaging is deeply

2 Since space is at a premium when writing text messages (which are limited to 160 characters), the truncation/rewriting of words to fit in the space allotted for the text message - as well as to save the time it takes to write the message and the money charged per message - becomes necessary. See "French Texting Rules" in Appendix B: "The basic rule of [French] texting is to express yourself with the fewest number of characters possible." 
embedded in the fabric of everyday life. Look around you: head-bowed, thumb-poised 'textperts' are everywhere. They have been credited with developing a new shorthand language, vowel-poor, acronym-rich, and emoticon-laden that is largely unfathomable to older people" (Patterson 2013: 84). Beyond demonstrating the quotidian prevalence of text messaging, however, these two films further serve to demonstrate texting's often vexed relationship with and impact upon the French language, such as in its creation of neologisms. In this comparative analysis, I examine text messaging and the use of SMS language as diegetic motor as well as tool for socio-cultural/linguistic innovation - in particular exemplary instances of SMS language such as the eponymous acronym/initialism "LOL" and the nonabbreviated spelling/syllabogram (or rebus writing, cf. Jacques Anis) "K LIN" (as a substitute for "câlin"/ "hugs") in LOL (see Figure I), and the neologism/abbreviation "opé" in De rouille et d'Os (see Figure 2) - as the common thread linking these two film narratives.

LOL (Laughing Out Loud) ${ }^{\circledR}$ is the coming of age story of 16 -year-old Parisian lycéenne Lola (Christa Théret) and her circle of friends, which includes her former boyfriend Arthur (Félix Moati) - who becomes verbally and physically abusive toward her after their relationship ends - and her new love interest, her longtime friend Maël (Jérémy Kapone). Lola is referred to by her nickname "Lol", a moniker that repeats and completes the SMS/text language embedded within the film's very title: Lol/LOL becomes a triple inscription signifying at once the film's female protagonist (Lol), its title ( $L O L)$, as well as the concept/meaning of "LOL" - as the film's subtitle indicates, "laughing out loud" - a universal notion that transcends linguistic boundaries: LOL reverberates, worldwide, as a ubiquitous and universally recognized/understood term employed in all forms of electronic and new media communication (email, internet chatting, SMS/text messaging, Twitter, Facebook, Skype, etc). ${ }^{3}$ Also worth noting is that, although it is a French film, $L O L$ 's title is written in two languages other than French: the SMS/text message term "LOL" followed by its standard/universal definition (the English "Laughing Out Loud") rather

3 For example, "LOL" was nominated for "Word of the Year" by the Danish television program Boogie (Jessen 2009, I7). 
than its French counterpart ("MDR"/ "mort de rire"4 or "to die of laughter"). According to Anis (2007), LOL "is not an acronym in the French context," although he notes that the French "MDR" "is very frequent in chat but is used less in SMS messages".

As opposed to the overall lightheartedness of $L O L,{ }^{5}$ De Rouille et d'Os is a tragic, haunting, and redemptive tale of life bringing together two emotionally and physically damaged, disillusioned protagonists who initiate an intimate relationship with each other: Oscar winner Marion Cotillard stars as Stéphanie, an attractive, formerly coquettish woman facing life alone after the loss of her legs in a horrific and violent incident at the amusement park where she trained killer whales, and Ali (Matthias Schoenharts, awarded the César for Most Promising Actor for his performance in this film), a single father struggling to support his young son by working as a security guard and making extra cash as a boxer in underground fighting rings. Text messaging plays a crucial role in the consummation of the physical relationship between Stéphanie and Ali: the word "opé" -short for "opérationnel" ("ready") - sent in a text message functions as their personal code for scheduling an intimate rendez-vous (the text messages consisting of the word "opé" and their emotional significance for De rouille et d'os are discussed below) (see Figure 2).

The films differ in terms of their locations - starring French cinema icon Sophie Marceau as the mother of the film's titular female teenage protagonist, $L O L^{6}$ is set in the world of French students at an upscale lycée, le Lycée Jean-Baptiste-Say in Paris' chic I6ème arrondissement, a milieu populated by wealthy French families (including the son of a government minister who is chauffeured to school) whereas De Rouille is situated in

4 See Cougnon and Beaufort (2009: 4I); Anis (2007).

5 Although the film also, to a certain extent, addresses (or at least, briefly presents) heavier topics such as bullying, child abuse, children coping with the divorce of their parents, teenage drug use, and students protesting against teachers who mistreat them.

6 In a reversal of Marceau's breakthrough role as rebellious adolescent protagonist Vic Beretton of the classic 1980 s teenpic La Boum/The Party (Claude Pinoteau, 1980), in LOL (Laughing Out Loud) ${ }^{\circledR}$, she plays the mother of a teenage daughter whom she attempts to shield from the temptations and pitfalls of growing up too fast. 
the grittier and less fashionable neighborhoods of the southern French beach town of Antibes, in the shadows of the glamourous resorts with which it is synonymous.

Despite these differences, upon closer viewing/reading, the films converge at several key points. These two narratives articulate their respective protagonists' quests for love and search for acceptance through finding meaning in their lives. Most significantly for our purposes in this discussion, the medium of text messaging occupies a primary position in each film in terms of the development of personal relationships between characters: romantic in both films, and also, in the case of $L O L$, other types of close relationships such as those of friends and family members. When the small, close-knit group of teenage characters in $L O L$ text each other and the couple in De Rouille et d'Os express their intimate desire for each other via text messaging, they reinforce what sociolinguistic research has discovered about texting in France, where texters "communicate predominantly with family or those in their innermost circle" (Thurlow and Poff 20II: 4) such as "close or intimate friends. Best friends and/or couples are the two relationships that are privileged with respect to the use of SMS," (Rivière and Licoppe 2005: np), as opposed to, for instance, SMS usage in Japan where "mobile messaging is not seen as acceptable in such [amorous/ romantic] contexts" (Rivière and Licoppe 2005: np).

Since text messaging is represented/treated in each film narrative at the levels of language, culture and meaning, $L O L$ and De Rouille demonstrate how texting "is the latest manifestation of the human ability to be linguistically creative and to adapt language to suit the demands of diverse settings. In texting we are seeing, in a small way, language in evolution" (Crystal 2008: 175) or "the view of French as a living language" (Anis 2007: np). According to Cougnon and Beaufort, in their work on building a French SMS to Standard Language Dictionary, "when dealing with SMS, one has to cope with various issues: new linguistic phenomena, language processing difficulties and lexical resource limits. Linguistic phenomena in SMS go from phonetic and numeral scripts, abbreviations and capital letters, to intensive use of neologisms, language mixing and borrowing, through new code systems such as emoticons" (2009:33), all of which are evidenced in the text messages used and represented in $L O L$ and De Rouille et d'Os. 
These films showcase several sociolinguistic aspects of text messaging specific to French texters and texting in France, where "SMS is perceived ... as completely different from any other form of electronic communication ... as a rather singular form of communication" (Rivière and Licoppe 2005: np).

Both films support linguistic research about text messaging which finds that "in France the use of SMS is reserved to a core of close correspondents, and involves emotional bond management", according to Rivière and Licoppe (2005: np), in terms of romantic as well as family relationships. In France, "a SMS is a sign of emotional attention that particularly befits amorous relationships, where the other person is considered a very special interlocutor ... In France, SMS messages are mostly sent to close or intimate friends. Best friends and/or couples are the two relationships that are privileged with respect to the use of SMS, independent of age" (Rivière and Licoppe 2005: np), as seen in both LOL and De Rouille.

Not only does the texting in these two films, in which SMS is the communication medium reserved for close friends, family members and lovers, support Rivière and Licoppe's notion of the emotional/small group aspect of text messaging in France, but also, it brings up some of the general linguistic phenomena related to SMS language as discussed above by Cougnon and Beaufort in their work on the French SMS, specifically relating to and engaging with the films' French language context. The use of text messaging in these films demonstrate how, according to Crystal, "French texters rely more on written language, abbreviated or full, and make more use of word-play" (2008: 147) than do texters in other languages. Furthermore, according to Crispin Thurlow and Michele Poff, citing the research of Anis (2007) and Rivière and Licoppe (2005), "French texters use phonetic reductions, syllabograms or rebus writing (e.g. as with the English $b_{4}$ for 'before'), and logograms which are symbols, acronyms, and unilateral abbreviations and reduce spoken forms to writing" (2011:5). One such example in $L O L$ is the use of "T ou" ("Where $\mathrm{r} u$ ") in a text message as a substitution for "Tu es où?" (see Appendix B for a list of common French text abbreviations as well as rules and patterns).

In representing how their French adolescent and adult protagonists reinvent the French language to communicate via text messaging - whether 
through creating neologisms such as the abbreviation "opé" in De Rouille or engaging in clever word play such as "K LIN" as a substitute for the French word "câlin" (hugs) in LOL - LOL and De Rouille et d'Os serve to showcase the often radically and disruptively creative potential and innovative aspect of texting in French. The texting in these films thus oppose the claims that text messaging is "un nouvel outil de destruction de la langue" (Cougnon 20II: 189) or a mechanism that "corrupts all languages" (The Economist 2008), a concern especially relevant for France: "the French are touchy because theirs [language] is so much an emblem of national identity" (The Economist 2008). Indeed, according to Jacques Anis, "more strictly than for other European languages, the rules of French orthography are considered to be absolute law. Spoken language forms are typically not acceptable in writing" since "France has long been known for its language academy ... and for preoccupations with linguistic prescriptivism and language purism" (Anis 2007: np).

In his analysis of neography in French SMS messages and its sociolinguistic impact, Anis (2007) finds that

it is not surprising that the abbreviated and often nonstandard orthography and grammar used in SMS messages have provoked the ire of [French] language purists, both online and offline. SMS spellings are controversial or prohibited in some French newsgroups. There is even a comité de lutte contre le langage SMS et les fautes volontaires (committee fighting against SMS language and deliberate errors).

Rather than corrupting French linguistic identity, however, in these two films, text messaging is represented as a means of opening a space allowing for the verbal expression of marginalized or Otherized characters finding their place in this world: in $L O L$, teenagers on the cusp of independence, rebelling against their parents and teachers while navigating the complex and difficult path to adulthood and its own rules, responsibilities, heartache and discontents (for further discussion of the "otherization"7 of

7 Adults' "hegemonic view of adolescents as immature, irresponsible, and deviant sets their language use up as problematic" (Eckert 2003: II6), ultimately "otherizing" them (Eckert 2003: I16). 
adolescents by "the ubiquitous adult gaze and the construction of moral panics around the behavior of" as well as the language used by teenagers, see Eckert 2003: 116); in De rouille et d'os, adult characters, who exist at the fringes of society, struggle with challenges and obstacles preventing self-actualization: Stéphanie's traumatic loss of bodily integrity and the poverty against which Ali struggles, breaking the law and sacrificing his own bodily integrity as a boxer in dangerous and illegal underground fights in order to earn money to support his son, for whom he is shown stealing food early in the film. For the adolescents of $L O L$ who chafe against their parents' and teachers' dictates and rules - including proper grammar and vocabulary ${ }^{8}$ - text messaging functions as their truest, purest means of expressing themselves to each other and even to their families with whom they are in conflict (such as Lola and her mother, discussed below). This evokes Thurlow and Poff's claim that "many of the typographic practices of texting offer more 'correct,' more 'authentic,' representations of speech to begin with ... in their messages texters 'write as if saying it' to establish a more informal register, which in turn helps to do the kind of small talk and solidarity bonding they desire for maximizing sociality" (20II: II). It is precisely this spontaneous quality that allows text messaging to innovate language, according to Cougnon and Beaufort: "l'écrit sms sera donc caractérisé comme suit: un écrit souvent spontané et familier, presque toujours créatif et ludique, autant de critères qui encouragent à la formation de mots et de sens nouveaux" (2011 : I9I).

Moreover, "French users are very sensitive to the use of SMS to express feelings. Because of the asynchronous nature of text messaging and the lack of interactional cues, it is evident that the medium can free the author from many inhibitions and modesties in expressing his or her emotions," (Rivière and Licoppe 2005: $\mathrm{np}$ ) recalling Cougnon and Beaufort's claim

8 Such as in the first classroom scene in the film, during which one of Lol's friends is castigated by their teacher for not knowing the correct meaning of a French word and thus "provokes the ire of a language purist" (Anis 2007), recalling how "France has long been known for its [...] preoccupations with linguistic prescriptivism and language purism" (Anis 2007). 
that texting "a tendance à inhiber les peurs traditionelles face à la communication" (2011: I9I).

"In France, the sentimental value of SMS stems mostly from the way users will play with the standard conventions of writing to maximize the meaningfulness of a given message for his intended interlocutor" (Rivière and Licoppe 2005). The emotional/sentimental quality of texting as discussed by Rivière and Licoppe is evidenced in both LOL and De Rouille, in which text messaging is closely associated with romantic relationships, friendships and between loved ones such as family members.

For example, in several key scenes of the film $L O L$, after Lola and her mother Anne fight, they subsequently make up after $\operatorname{Lol}(\mathrm{a})$ texts her mother "K LIN," a substitution for the French word "câlin" ("hugs") (see Figure I). In the sequence that immediately follows, Lola and Anne are shown hugging and cuddling together, clearly having reconciled after their disagreement. Note that the SMS word, "K LIN," is rendered untranslatable by the film's English subtitles which only give blank parentheses. In his 2007 "Neography: Unconventional Spelling in French SMS Text Messages", Jacques Anis discusses the substitution of "c" by " $k$ " in French text messaging as a form of nonabbreviated spelling. "K LIN," would be, according to Anis, an example of a "syllabogram or rebus writing": "the use of a letter or a number to represent the phonetic sequence that constitutes its realization in spoken language" (2007). The SMS/text message medium, platform and language thus prefigure and arguably allow for mother-daughter reconciliation at crucial moments in the film $L O L$, culminating in their physical act of hugging, upon which the film ends (in a reversal of the earlier "K LIN" scene, by the film's end, it is Lol's mother who initiates their reconciliation by contacting Lol over instant message computer chat, after which they are, again, immediately shown hugging each other, as the film lingers on its final word, "lol," before fading to black).

SMS language and text messaging leads to a physical encounter of a much different sort for Stéphanie and Ali in De Rouille et d'Os. The private word they create and text to each other, "opé", is the lovers' SMS code for scheduling a rendez vous to physically consummate their relationship, allowing for the flourishing of their sexual and romantic relationship that eventually leads them both on a redemptive journey which culminates 
in healing: with the assistance of prosthetic legs and a cane, Stéphanie is able to walk, and, moreover, finds the peace and self-acceptance which she lacked at the start of the film when she was physically "whole" yet seeking to fill her emotional void by flaunting her alluring figure at nightclubs in order to provoke men into fighting over her and sparking her boyfriend's jealousy. By the film's end, she has found the love and acceptance of Ali and his son, and furthermore, is fulfilled by her new occupation as Ali's manager. So too has Ali improved his life: no longer participating in illegal fighting, but after rigorous training and discipline he becomes a legitimate, successful boxing champion, capable of loving and caring for Stéphanie and his son.

Together, Ali and Stéphanie are fully "opé” ("operational”) by the film's end, although when they initially begin texting "opé" to each other, they were anything but: Stéphanie was severely depressed as she tried to learn to live with her amputated limbs. Ali was incapable of accepting the responsibilities of single fatherhood and was often shown mistreating his young son culminating in a violent outburst when he cannot soothe his son's crying, after which his sister removes the frightened boy from his care. It is Ali who creates the SMS abbreviation/neologism "opé," explaining its meaning to Stéphanie after they first make love at her request since after the accident and amputation of her legs she feels unattractive and afraid of engaging in sexual intercourse with a man, a fear which Ali helps her overcome.

Their private lovers' language and carnal pleasure is thus mediated through the language of text messaging. They create a new vocabulary, a new abbreviation/term, "opé," which they use to refer to scheduling their lovers' trysts. If she texts him "opé" and he is available, he will answer the call, so to speak, which culminates in their physical consummation.

In a later sequence in the film, the blossoming of Stéphanie's feelings for Ali - and her newfound zest for life, despite the double amputation of her legs - is again inextricably intertwined with the cell phone and text messaging: she surreptitiously texts him, using their secret word "opé," while interacting with her friends who are oblivious to what she is doing. She contacts him with her phone, hiding it under the table while she texts him, all the while engaging in conversation with her work friends (see Figure 2). This sequence serves to highlight one of the benefits of communicating 
by text message, namely, "that the messages can be sent quietly and discretely" (McKay et al. 2005: 196). This scene moreover illustrates a specific aspect of SMS to the French language context of the film, since, according to Crystal, in France, "texts are seen as a way of managing privacy in a public space, allowing communication while maintaining a silent presence" (2008: I 47). Through surreptitiously texting Ali while interacting with her colleagues, Stéphanie negotiates the boundary between the public and private spheres: she can communicate her private desire for meeting with Ali while maintaining a silent, public façade that reveals absolutely nothing about their imminent erotic encounter (which occurs in the sequence immediately following this one).

Stéphanie and Ali express their most intimate desire for each other via one SMS word, "opé," whose meaning shifts as their relationship moves from the purely physical encounters they schedule with each other to a substantial one of love and the creation of a new family. By the end of the film, their relationship has been transformed, becoming one of mutual commitment and caring, although it began as a means to gratify a sexual urge, no more, no less. Thus they have redefined the term "opé"; its meaning is shifting: from the "opé" of the "no strings attached booty call" to the emotional and physical operationality required for a committed romantic relationship, which is how the film concludes: with the success of Ali as a boxer due to Stéphanie's management of his career, their love flourishes, as does her role as a surrogate mother figure to Ali's son, whose biological mother has abandoned him. After (accidentally) exposing the boy to - and rescuing him from - a near-fatal accident, Ali is now a responsible father fully devoted to caring for his son.

De Rouille therefore moves from the language of a text message signifying a "hook up" / "booty call" - in other words, a physical encounter without emotional ties - to a thriving career and family unit for Stéphanie, Ali and his son. The text message containing the SMS neologism/abbreviation "opé" functions as the instigator for this new chapter in all of their lives. The creation of this new word ultimately paves the way to a new life in which adult responsibilities of work, parenthood, and emotional maturity are not only accepted, but also, greatly enjoyed. Ironically it is the text message, considered an "adolescent" means of communication, which 
allows Stéphanie and Ali to mature and move beyond their previous states of immaturity and irresponsibility, working through their dysfunction and trauma from which they ultimately free themselves and reach selfactualization (recalling how in LOL, Lola and her mother put aside their differences and reconcile via text and instant messaging).

In both LOL (Laughing Out Loud) ${ }^{\circledR}$ and De Rouille et d'Os, therefore, it is not so much a question of harm to the French language that is raised by the significant presence of text messaging in both film's diegeses, but rather, how SMS functions as a means of linguistic innovation and of communication of deep expressions of love, intimacy and the maintenance of "emotional bonds" between French-speaking texters - whether between family members or a couple in a relationship - demonstrating that for French texters, "the written [SMS] message is therefore often compared to a love letter that one keeps and reads over and over" (Rivière and Licoppe 2005: np).

\section{References}

Anis, Jacques, 2007, "Neography: Unconventional Spelling in French SMS Text Messages", in Danet, Brenda \& Herring, Susan (eds), The Multilingual Internet: Language, Culture, and Communication Online, 87-115. Kindle version.

Cougnon, Louise-Amélie \& Beaufort, Richard, 2009, "SSLD: a French SMS to Standard Language Dictionary", in Proceedings of eLexicography in the $2 I^{t t}$ century: New Applications, New Challenges (eLEX 2009), 33-42.

Cougnon, Louise-Amélie \& Beaufort, Richard, 20II, « Néologie et sms », Neologica: Revue internationale de néologie, 5, I83-20I.

Crystal, David, 2008, Txting: The Gr8 Deb8, Oxford, Oxford University Press.

Eckert, Penelope, 2003, "Language and Adolescent Peer Groups," in Journal of Language and Social Psychology, 22, II2-II8.

Jessen, Mia Kjær, 2009, « Le langage chat et SMS est-il une menace pour l'usage traditionnel de la langue? », BA Thesis, Aarhus School of Business.

McKay, Susan, Thurlow, Crispin \& Zimmerman, Heather, 2005, "Wired Whizzes or Techno-Slaves? Young People and Their Emergent Communication 
Technologies", in Williams, Angie \& Thurlow, Crispin (eds), Talking Adolescence: Perspectives on Communication in the Teenage Years, New York, Peter Lang, $185-203$.

Patterson, Anthony, 20I3, "Digital Youth, Mobile Phones and Text Messaging: Assessing the Profound Impact of a Technological Afterthought," in Belk, Russell \& Llamas, Rosa (eds), The Digital Consumer, London, Routledge, 83-92.

Reid, Donna \& Fraser, Reid, February 2004, "Insights into the Social and Psychological Effects of SMS Text Messaging", <http://courses.educ.ubc.ca/etec540/ Mayo8/suz/assests/SocialEffectsOfTextMessaging.pdf>

Rivière, Carole Anne \& Licoppe, Christian, 2005, "From Voice to Text: continuity and change in the use of mobile phones in France and Japan", in Harper, Richard, Palen, Leysia \& Taylor, Alex (ed), The Inside Text: Social, Cultural, and Design Perspectives on SMS, Berlin, Springer, 103-126. Kindle version.

Thurlow, Crispin, 2003, "Generation Txt? The sociolinguistics of young people's textmessaging”, <http://extra.shu.ac.uk/daol/articles/vi/nI/a3/thurlow2002003paper.html $>$

Thurlow, Crispin \& Poff, Michele, 20II, "Text Messaging." < http://www.crispinthurlow.net/papers/thurlow\&poff(2010).pdf>

"Txt Msgng in Frnc: Parlez-vous SMS? A new Threat to the French Language," The Economist, 22 May 2008, <http://www.economist.com/node/II4I2629>.

\section{Filmography}

De Rouille et D'Os (2012), Jacques Audiard, dir., France 2 Cinema. LOL (Laughing Out Loud) ${ }^{\circledR}$ (2008), Lisa Azuelos, dir., Pathé. 


\section{Appendix A: Figure I and Figure 2: Screengrabs from $L O L$ (Laughing Out Loud) ${ }^{\circledR}$ and De Rouille et d'Os, respectively}

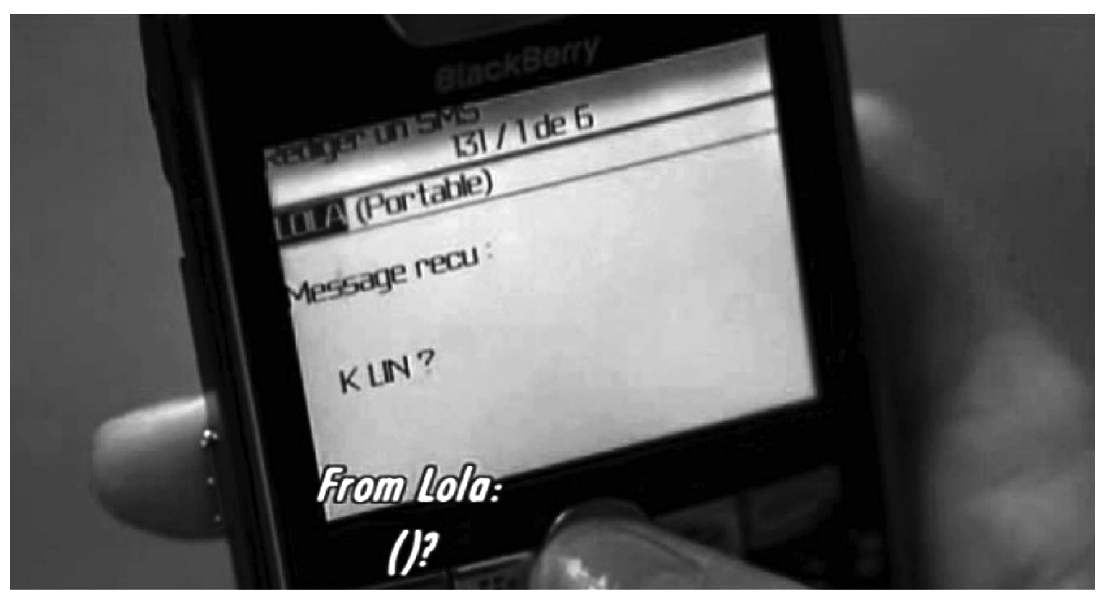

Figure I: In Lisa Azuelos’ 2008 film LOL (Laughing Out Loud) ${ }^{\circledR}$, after a disagreement with her mother, teenage protagonist Lola texts "K LIN" - a substitution for the French word "câlin" ("hugs") - to her mother. In the next scene, mother and daughter are shown hugging; Lola's message to her mother and their subsequent reconciliation emphasizes how "in France, the sentimental value of SMS stems mostly from the way users with play with the standard conventions of writing to maximize the meaningfulness of a given message for his intended interlocutor" (Rivière and Licoppe 2005). Note that the French SMS term "K LIN" is rendered untranslatable by the film's English subtitles, which represent it as “()." For further discussion of the substation of "c" by "k" as a nonabbreviated spelling in French text messaging, see Anis (2007). "K LIN" would be, according to Anis (2007), an example of a "syllabogram or rebus writing": "the use of a letter or a number to represent the phonetic sequence that constitutes its realization in spoken language." For further information about the letter $\mathrm{K}$ as a replacement for QU and CA in French text messaging, see Appendix B (below).

$$
\text { Images (C) } 2008 \text { Pathé }
$$



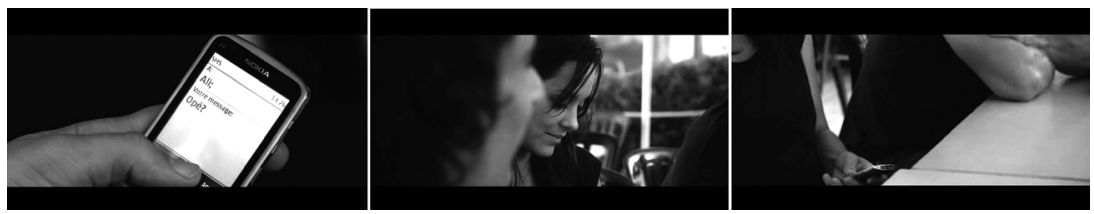

Figure 2: In Jacques Audiard's De Rouille et d'Os (2012), while spending time with her friends, Stéphanie surreptitiously texts opé' ("operational”) to Ali, scheduling a rendez vous with him (shown in the next scene). By hiding the phone under the table and away from her friends while she texts Ali, Stéphanie negotiates the boundaires of the public and private spheres, recalling that according to David Crystal (2008, I47), in France, "texts are seen as a way of managing privacy in a public space, allowing communication while maintaining a silent presence."

Images (C) 2012 Why Not Productions

\section{Appendix B: Common French Text Abbreviations and Rules}

Table of Abbreviations

\begin{tabular}{|c|c|c|}
\hline French & Meaning & English \\
\hline I $2 \mathrm{C}_{4}$ & un de ces quatre & one of these days \\
\hline 2 ri I & de rien & you're welcome \\
\hline 6né & Ciné & Movie theater \\
\hline $\begin{array}{l}\text { A+ } \\
@+\end{array}$ & À plus & $\begin{array}{l}\text { L8R, later } \\
\text { CUL8R, see you later }\end{array}$ \\
\hline $\mathrm{A}_{12} \mathrm{C}_{4}$ & À un de ces quatre & See you one of these days \\
\hline $\begin{array}{l}\mathrm{a} 2 \mathrm{mI}_{\mathrm{I}} \\
@ 2 \mathrm{mI}\end{array}$ & À demain & $\mathrm{CU}_{2}$ moro, see you tomorrow \\
\hline ALP & À la prochaine & TTFN, ta ta for now \\
\hline AMHA & À mon humble avis & $\begin{array}{l}\text { IMHO, in my humble } \\
\text { opinion }\end{array}$ \\
\hline
\end{tabular}




\begin{tabular}{|c|c|c|}
\hline $\begin{array}{l}\text { AP } \\
\text { APLS }\end{array}$ & À plus & TTFN, ta ta for now \\
\hline ASV & Âge, Sexe, Ville & ASL, age, sex, location \\
\hline $\mathrm{att}$ & à tout à l'heure & see you soon \\
\hline auj & Aujourd'hui & Today \\
\hline bisur & Bien sûr & Of course \\
\hline BAL & Boîte aux lettres & Mailbox \\
\hline $\mathrm{BCP}$ & Beaucoup & A lot \\
\hline bi ito & Bientôt & RSN, real soon \\
\hline biz & bisous & Kisses \\
\hline bjr & Bonjour & Hello \\
\hline bsr & Bonsoir & Good evening \\
\hline $\mathrm{C}$ & C'est & It is \\
\hline Ci Blag & C'est une blague & It's a joke, Just kidding \\
\hline CAD & C'est-à-dire & That is, i.e., \\
\hline $\mathrm{cb}_{\mathrm{I}}$ & C'est bien & That's good \\
\hline C cho & C'est chaud & It's hot \\
\hline Cé & C'est & It is \\
\hline Ché & $\begin{array}{l}\text { Chez } \\
\text { Je sais }\end{array}$ & $\begin{array}{l}\text { At the home of } \\
\text { I know }\end{array}$ \\
\hline $\begin{array}{l}\text { Chu } \\
\text { Chui } \\
\text { Chuis }\end{array}$ & Je suis & I am \\
\hline $\mathrm{C}$ malı & C'est malin & That's clever, sneaky \\
\hline C pa spa & C'est pas sympa & That's not nice \\
\hline CPG & C'est pas grave & INBD, it's no big deal \\
\hline $\mathrm{Ct}$ & $\begin{array}{l}\text { C'était } \\
\text { C'est tout }\end{array}$ & $\begin{array}{l}\text { It was } \\
\text { That's all }\end{array}$ \\
\hline Dioo & Descends & Get down \\
\hline
\end{tabular}




\begin{tabular}{|c|c|c|}
\hline $\begin{array}{l}\text { d'ac } \\
\text { dak }\end{array}$ & D'accord & OK \\
\hline DSL & Désolé & IMS, I'm sorry \\
\hline DQP & Dès que possible & ASAP, as soon as possible \\
\hline EDR & Écroulé de rire & LOL, laughing out loud \\
\hline $\begin{array}{l}\text { ENTK } \\
\text { EntouK }\end{array}$ & En tout cas & IAC, in any case \\
\hline FAI & Fournisseur d'accès internet & ISP, internet service provider \\
\hline FDS & Fin de semaine & WE, Wknd, weekend \\
\hline G & J'ai & I have \\
\hline Grid2kdo & J'ai une idée de cadeau & I have a great idea \\
\hline GHT & J'ai acheté & I bought \\
\hline $\mathrm{GHT}_{2} \mathrm{~V}_{\mathrm{I}}$ & J'ai acheté du vin & I bought some wine \\
\hline G la N & J'ai la haine & $\mathrm{H} 8$, hate \\
\hline GspR b I & J'espère bien & I hope so \\
\hline Gt & J'étais & I was \\
\hline Jé & J'ai & I have \\
\hline Je c & Je sais & I know \\
\hline Je le saV & Je le savais & I knew it \\
\hline Jenémar & J'en ai marre & I'm sick of it \\
\hline Je t'M & Je t'aime & ILUVU, I love you \\
\hline $\begin{array}{l}\text { Je vé } \\
\text { J'vé }\end{array}$ & Je vais & I’m going \\
\hline JMS & Jamais & NVR \\
\hline JSG & Je suis génial & I'm (doing) great \\
\hline JTM & Je t'aime & I love you \\
\hline $\mathrm{K}_{7}$ & Cassette & cassette tape \\
\hline
\end{tabular}




\begin{tabular}{|c|c|c|}
\hline KDO & Cadeau & Gift \\
\hline $\begin{array}{l}\text { Kan } \\
\text { Kand }\end{array}$ & Quand & When \\
\hline $\mathrm{Ke}$ & Que & that, what \\
\hline Ké & Qu'est & What is \\
\hline Kel & Quel, Quelle & Which \\
\hline Kelle & Qu'elle & That she \\
\hline Keske & Qu'est-ce que & What \\
\hline $\begin{array}{l}\text { kestufou } \\
\text { Ksk t’fu }\end{array}$ & Qu'est-ce que tu fous? & What the hell are you doing? \\
\hline $\mathrm{Ki}$ & Qui & Who \\
\hline Kil & Qu'il & That he \\
\hline Koi & Quoi & What \\
\hline Koi29 & Quoi de neuf? & What's new? \\
\hline Lckc & Elle s'est cassée & She left \\
\hline L's tomB & Laisse tomber & Forget it \\
\hline Lut & Salut & $\mathrm{Hi}$ \\
\hline M & Merci & Thanks \\
\hline MDR & Mort de rire & ROFL \\
\hline $\mathrm{mr} 6$ & Merci & Thx, thanks \\
\hline MSG & Message & Msg, message \\
\hline now & Maintenant & ATM, at the moment \\
\hline NSP & Ne sais pas & Dunno \\
\hline o & $\mathrm{Au}$ & In the, at the \\
\hline Oki & Aucun & None, not one \\
\hline OQP & Occupé & Busy \\
\hline Oué & Ouais & Yeah \\
\hline
\end{tabular}




\begin{tabular}{|c|c|c|}
\hline p2k & Pas de quoi & URW, you're welcome \\
\hline parske & Parce que & COZ, because \\
\hline $\begin{array}{l}\text { p-ê } \\
\text { pitit }\end{array}$ & Peut-être & Maybe \\
\hline $\mathrm{PK}$ & Parce que & Because \\
\hline Pkoi & Pourquoi & Y, why \\
\hline $\begin{array}{l}\text { Po } \\
\text { Pô }\end{array}$ & Pas & Not \\
\hline PTDR & Pété de rire & $\begin{array}{l}\text { ROFLMAO, rolling on the floor } \\
\text { laughing }\end{array}$ \\
\hline $\begin{array}{l}\text { q-c q } \\
\text { queske }\end{array}$ & Qu'est-ce que & What \\
\hline QDN & Quoi de neuf? & What's new? \\
\hline qq & Quelques & Some \\
\hline qqn & Quelqu'un & Someone \\
\hline raf & Rien à faire & Nothing to do \\
\hline ras & Rien à signaler & Nothing to report \\
\hline $\mathrm{rdv}$ & Rendez-vous & Date, appointment \\
\hline $\mathrm{RE}$ & (Je suis de) retour, Rebonjour & I'm back, $\mathrm{Hi}$ again \\
\hline ri I & Rien & o, nothing \\
\hline savapa & Ça va pas? & Is something wrong? \\
\hline SLT & Salut & $\mathrm{Hi}$ \\
\hline SNIF & J'ai de la peine & I'm sad \\
\hline ss & (je) suis & I am \\
\hline STP/SVP & S'il te/vous plaît & PLS, please \\
\hline $\mathrm{T}$ & T'es & You are \\
\hline tabitou & T'habites où? & Where do you live? \\
\hline
\end{tabular}




\begin{tabular}{|l|l|l|}
\hline tata KS & T'as ta casse? & You have your car? \\
\hline tds & tout de suite & right away \\
\hline tiz & T'es hideux & You're hideous. \\
\hline tjs & Toujours & Always \\
\hline tkc & T'es cassé & You're tired. \\
\hline TLM & Tout le monde & Everyone \\
\hline T nrv? & T'es énervé? & Are you irritated? \\
\hline TOK & T'es OK? & RUOK? Are you OK? \\
\hline TOQP & T'es occupé? & RUBZ? Are you busy? \\
\hline tps & temps & time, weather \\
\hline $\begin{array}{l}\text { Tt } \\
\text { tt }\end{array}$ & $\begin{array}{l}\text { T'étais } \\
\text { tout }\end{array}$ & $\begin{array}{l}\text { You were } \\
\text { all, every }\end{array}$ \\
\hline \multicolumn{2}{|l|}{} \\
\hline VI & Viens & Come \\
\hline vazi & Vas-y & Go \\
\hline VrMan & Vraiment & Really \\
\hline \multicolumn{2}{|l|}{} \\
\hline X & crois, croit & Believe \\
\hline XLnt & Excellent & XLNT, excellent \\
\hline \multicolumn{2}{|l|}{} \\
\hline $\begin{array}{l}\text { y a } \\
\text { ya }\end{array}$ & $\begin{array}{l}\text { Il y a } \\
\text { There is, there are }\end{array}$ \\
\hline
\end{tabular}

French Texting Rules

The basic rule of texting is to express yourself with the fewest number of characters possible. This is done in three ways:

Using abbreviations, like TLM for Tout Le Monde

Using letters that are pronounced like the desired sounds, like $O Q P$ for occupé (O - CCU - PÉ)

Dropping silent letters, especially at the end of a word, like parl for parle 


\section{Patterns}

- I replaces UN, EN, or IN

- 2 replaces DE

- C replaces C'EST, S'EST, SAIS, etc.

- É replaces AI, AIS, and other spellings of similar sounds

- K can replace QU (e.g., koi) or CA (kdo)

- O replaces AU, EAU, AUX, etc.

- T replaces T'ES and other spellings of the same sound

Source: "French Texting - Les textos français," http://french.about.com/library/ writing/bl-texting.htm. (See also David Crystal, Txting: The Grs Deb8 (Oxford University Press, 2008), pp. 207-2 I I, for a list of French text abbreviations). 


\section{Notices biographiques}

MICHAËL ABECASSIS is Senior Instructor in French and a college lecturer at the University of Oxford. He has published widely on French Linguistics and Cinema. His publications include: The Representation of Parisian Speech in the Cinema of the I930s (Peter Lang, 2005); Le français parlé au XXIème siècle, in two volumes (L'Harmattan, 2008) with Laure Ayosso and Elodie Vialleton; Les Voix des Français, in two volumes (Peter Lang, 2010) with Gudrun Ledegen; Écarts et apports des médias francophones: Lexique et grammaire (Peter Lang, 2013) with Gudrun Ledegen; French Cinema in Close-up - La Vie d'un acteur pour moi (Phaeton, forthcoming), with Marcelline Block; Confident French: A Dictionary of Contemporary usage (Phaeton, forthcoming).

BÉATRICE AKISSI BOUTIN est membre de l'Institut de Linguistique Appliquée de l'Université Félix Houphouët-Boigny d'Abidjan, et chercheur associée au CLLE/ERSS-UMR 5263, Université Toulouse Le Mirail Jean Jaurès. Elle développe ses recherches essentiellement dans la zone ouestafricaine, notamment en Côte d'Ivoire. Ses domaines d'intérêt concernent la dynamique de la variation et des répertoires linguistiques plurilingues. La primauté est donnée à l'analyse des faits phonologiques et syntaxiques de français et des principales langues en contact avec le français en Côte d'Ivoire (dioula, baoulé).

MARCELLINE BLOCK est diplômée de Harvard et de Princeton, spécialiste de cinéma. Auteur de nombreux textes et articles sur le cinéma français et francophone, parmi ses livres sont The Directory of World Cinema : Belgium (Intellect, 20I4), World Film Locations: Paris (Intellect, 20II), World Film Locations: Marseille (Intellect, 2013) et sa version française, Filmer Marseille (Presses universitaires de Provence, 2013). 
MARION BLONDEL est chercheure au CNRS-Paris8. Elle est linguiste spécialiste des langues des signes (en particulier de poétique et de prosodie) et s'intéresse plus généralement au langage en contexte de surdité (en particulier l'acquisition bilingue-bimodale LSF/ français, l'écrit-sms et la circulation interlinguistique dans les échanges en contexte de surdité). Elle a enseigné à Tours, Lille, Lyon, Rouen et codirige l'axe « Langues des signes et Gestualité » de la revue LIA (Ed. Maya Hickmann, chez Benjamins).

ASMA CHAMLY-HALWANI, professeur titulaire à l'Universite Libanaise. Chargée de cours et directrice d'une unité de recherche à L'Ecole Doctorale des Lettres et des Sciences Humaines et Sociales. Ex-doyenne de la Faculté des Lettres et des Sciences humaines, elle s'intéresse dans ses recherches aux études de l'imaginaire, de la religion dans la littérature, aux études comparatives ainsi quaux études historiques et interculturelles. Elle a publié de nombreuses études concernant des écrivains français, francophones, arabes, orientaux et européens tels que Gérard de Nerval, Marguerite Yourcenar, Richard Millet, Philippe Claudel, Amin Maalouf, Ghada El Samman, Umberto Eco, Orhan Pamuk, etc.

ANNE-CAROLINE FIÉVET est ingénieure de recherche à l'EHESS (Paris, France), elle est responsable des études avec les jeunes enfants et les bébés au laboratoire de sciences cognitives et psycholinguistique (LSCP). Après une double formation en linguistique et en sciences de l'information et de la communication, elle a soutenu, en 2008 , une thèse sur le lexique employé par les jeunes à la radio, dont Skyrock. Elle est membre du Groupe de Recherche et d'Études sur la Radio (GRER). Depuis 2005, Alena PodhornáPolická et Anne-Caroline Fiévet mènent des recherches communes sur la circulation des néologismes identitaires pour les jeunes. Par exemple, elles ont travaillé sur la circulation de ces néologismes dans les films, la littérature et les chansons de rap. Depuis 2006, elles suivent l'évolution du lexème «bolos », entré dans le Petit Robert en 2014.

FRANÇOIS GAUDIN, professeur de sciences du langage et docteur en histoire, est membre du laboratoire « Lexique, dictionnaires, informatique $\gg$ Paris XIII/CNRS. Après de nombreuses publications sur la 
socioterminologie, il développe et anime des recherches en histoire culturelle des dictionnaires. Il a dirigé Le monde perdu de Maurice Lachâtre (2006) et La lexicographie militante (2013) publiés par Honoré Champion. Il a édité avec Jean-Yves Mollier Cinq centimes par jour. Pratiques commerciales d'un éditeur engagé, PURH (2008). Il a dirigé Alain Rey, vocabuliste français, Ed. Lambert-Lucas (20II) et Au bonheur des mots. Hommage à Alain Rey, PURH (2014) ainsi qu'un cycle de conférences de vulgarisation Rumeur des mots, PURH (2013).

JEANNE GONAC'H est actuellement enseignante d'anglais au Lycée P. Corneille à Rouen. Son travail de doctorat a porté sur les pratiques et activités littératiées de jeunes bilingues en France et en Angleterre. Son travail s'inscrit essentiellement dans une approche sociolinguistique et les recherches qu'elle poursuit actuellement portent toutes sur les contacts de langue à l'écrit dans des contextes plurilingues.

SOU HEILA HEDID est enseignante universitaire de langue française à l'université Constantine r en Algérie. Elle explore depuis plusieurs années la situation sociolinguistique de la ville algérienne et ce à travers plusieurs problématiques : les pratiques langagières des jeunes, leurs représentations, les parlers urbains dans la ville arabe. Actuellement elle met l'accent sur la question de la mobilité sociospatiale dans les espaces urbains et la création des nouvelles villes.

GEMMA KING, doctorante en cotutelle à Paris 3 en France et l'Université de Melbourne en Australie, prépare sa thèse sur le multilinguisme et le pouvoir dans le cinéma français contemporain. Professeur de français à Melbourne et d'anglais à Paris, elle a publié ses recherches chez Linguistica Antverpiensia, The Australian Journal of French Studies, The Journal of the Anthropology of the Contemporary Middle East and Central Eurasia et Librairies Garnier Paris. Après la cotutelle, elle souhaite poursuivre son travail dans le domaine du cinéma français en analysant le rapport entre la langue, le pouvoir et la violence.

GUDRUn LEDEGEN est Maître de Conférences en Sciences du Langage à l'Université de Rennes 2 et auparavant à l'Université de La Réunion. Ses 
recherches portent sur la sociolinguistique, la syntaxe du français ordinaire et les langues en contact (français - créole réunionnais), entre autres dans les parlers jeunes, ou encore l'écrit-sms, en prenant appui sur le corpus d'oral et d'écrit ordinaires, Valirun (Variétés Linguistiques de La Réunion). Elle s'intéresse par ailleurs à la circulation interlinguistique dans les échanges en contexte de surdité. Elle a édité et dirigé plusieurs ouvrages, dont Les Voix des Français, en deux volumes (Peter Lang, 2010), Écarts et apports des médias francophones: Lexique et grammaire (Peter Lang, 2013) tous deux avec Michael Abécassis.

CHANTAL LYCHE est professeur de linguistique française à l'université d'Oslo et a été pendant de nombreuses années professeur adjoint à l'université arctique de Norvège. Elle est la co-directrice du programme «Phonologie du français contemporain » (PFC) qui met à la disposition des chercheurs un grand corpus oral transcrit, aligné et codé. Elle a publié extensivement sur la phonologie du français et s' intéresse particulièrement aux variétés hors de France. Ses travaux portent également sur la prosodie et la reconnaissance d'accent. Elle est la co-éditrice de plusieurs ouvrages sur la phonologie du français et sur des travaux issus du corpus PFC.

ALENA PODHORNÁ-POLICKÁ est maître de conférences à l'Université Masaryk (Brno, République tchèque) et membre associée au laboratoire PREFIcs (Université Rennes 2, France), elle s'intéresse à la sociolexicologie et à la sociolinguistique urbaine. Elle mène également des recherches dans le domaine des emprunts néologiques, de la traduction et de la lexicographie du substandard. Depuis 2009, elle créé une base de données textuelle de chansons de rap, le RapCor, afin d'en étudier le contenu linguistique.

JEAn PRuvost, Professeur à l'Université de Cergy-Pontoise (95), où il enseigne la lexicologie et Professeur d'Université, Directeur du laboratoire CNRS, Lexiques Dictionnaires Informatique de l'Université de CergyPontoise, Directeur éditorial des éditions Honoré Champion, Directeur des Études de linguistique appliquée, organise chaque année depuis 1993 la Journée des dictionnaires. Prix international de linguistique Logos (Dictionnaires et nouvelles technologies, PUF, 2000), Prix de l'Académie 
française (Les dictionnaires français : outils d'une langue et d'une culture, Ophrys, 2006), il est l'auteur de plus de 450 publications dont La Dent de lion, la Semeuse et le Petit Larousse (2005), un Dictionnaire de citations françaises (Bordas, 2007) et deux Que sais-je? (Dictionnaires; Néologie), le Journal d'un amoureux des mots (Larousse, 2103) et le Dico des dictionnaires, Histoire et anecdotes (Lattès, 2014). Dans la collection « Champion Les mots », il a publié successivement depuis 2010, Le Vin, Le Loup, Le Chat, La Mère, Le Fromage, Les Élections, Le Train, Le Jardin, Le cirque, À vélo ou à bicyclette, nom d'un tour, Le champagne. Il a tenu et tient diverses chroniques de langue radiophoniques (France Inter, Canal Académie, RCF).

CAROLINE Rossi, ancienne élève de l'École Normale Supérieure de Lettres et Sciences Humaines et agrégée d'anglais, est linguiste et Maître de Conférences à l'université Grenoble Alpes. Elle travaille sur l'acquisition du langage dans une perspective translinguistique, qu'il s'agisse du développement langagier chez l'enfant, ou de l'acquisition d'une langue seconde. Elle a enseigné à King's College (Londres) à Brasenose, Lincoln et Christ Church College (Oxford), et a bénéficié d'une bourse d'études de la Maison Française d'Oxford. Elle a édité un ouvrage collectif qui réunit linguistes, philosophes, psychanalystes, traducteurs, écrivains et poètes autour de la question de la (re)fabrication de la langue : Fabriques de la langue, publié par les Presses Universitaires de France.

NATHALIE ROSSI-GENSANE est professeur de Sciences du langage à l'Université Lumière Lyon 2, où elle enseigne la linguistique française, et membre du laboratoire ICAR (UMR 5I9I). Au sein de ses domaines de recherche que sont la syntaxe et la sémantique, le français écrit et le français oral, ses travaux portent plus particulièrement sur les constructions détachées en rupture, les problèmes de segmentation - à l'écrit et à l'oral - et les rapports entre grammaire et linguistique. 



\section{Index}

adjectif $3,17,19-20,37,39,65$ n.25, 137,

$$
\text { I 42, I44-I } 45
$$

adolescent 54 n.8, 56, 226, 244-245, 247 n.6, 249-25I, 255

adverbe $7 \mathrm{I}, 73-74,83$

apprentissage $15-22$

arabe et français $3-4$, III, I80-200, $20 \mathrm{I}-220,235-237$

attitude 32 n.6, 35, IO3 n.I, II 6

auxiliaires 3, I6, 23, I58, I64, I66-I67

cinéma I, 4-5, 22I-242, 243-264 conditionnel 3, 71, I58, I64-I67 corpus I-2, 9, I2, I4-I6, I8-I9, 22, 30, $32-33,40-41,44,49-70,72-76$, $8 \mathrm{I}, 85-87,94$, I27, I55 n.I, I57, I6I-I67, I69, I7 I, I8I-I82, I85-I86, I88, I92, 268

créole I, IO, I4, 84, 87 n.I2, 9I, 268

discours épilinguistique 5, I8I

école 78 n.5, I09, I42, I49, I8I, I87, I93, $228,236,244$

écrit 9, $12-16,19-24,29-45,54$ n.8, $55-56,59,64-65,78,83,90-93$, I3I, 245, 251

écriture 203, 206-207, 211-212, 215

enfant II, I5, I7-I9, 23, 35, 76, IO2, III, I37-I5I, I72, 232, 2354, 247 n.5, 266, 269

enseignement $16,76-77,106-108,116$, I42, 183,190

épenthèse I, II-I2, 23 grammaire $13,34,38,42,71,83$, 109, II2, I39-I40, I56, I59-160, I62 n.6, I67-I68, I7 I, I74, 193, 250-25I

graphie I, 9, I2-I3, 2I-22

histoire 2, 5, 38, IO4, I08-IIO, II3-I37, $202-203,205,207,212,215,225$, 231, 237

identité 2-3, 49-52, 54, 57, 59-6I, 66-67, 102, I83, 194, 202-208, 211, 216-219, 223, 225, 232, $237-238,244,250,256$

interrogation 3,143 n.7, I57-164, I82, I89, 212

lexicographie II8, I3I, I33 liaison facultative 24 liaison obligatoire 9-24 mots composés $137-139$

négation 39, 7I-93

non-standard $30 \mathrm{n} .3$

norme $12,30-31,36,42$ n.I0, I06-IIo, II2, I9I

passif 159

plurilinguisme $77,181-183,188,192$, 195-197, 202, 213

politique 107, 115-117, 119, 192, 215 pronoms (absence de $\sim$ ) 40, 4I, 8I, 92 prononciation 14,117 
que (absence/présence de ) I58, I69-I70, I73

radio 2, I2, 49-70, 74, I05, I33, I 49 représentation (sociolinguistique) 106, IIO-II2, I86-I87, I97, 205, 219

sémantique 33, 60, 75, 82, I37-I38, I49, I55, I57, I60, I70, I73, I94

SMS 29-34, 36-37, 39-4I, 43, 45, $50-56,63-65,68,72,83,87-93$, 244-255, 257

style $15,30,104,110,139$

société 31, 49, 52, 76, 107, I81, I83

stéréotype 49-50

subjonctif $3,158,164-165$

substantif I6-2I

surdité (écrits en contexte de ) 29-3I, 44-45 syntagme $82, \mathrm{II} 7,137-139, \mathrm{I} 4 \mathrm{I}-\mathrm{I} 42$, I 44-I45, I47, I7 I

syntaxe (morpho ) 29-31, 33, 43-44, 72, 9I-92, I37, I39, I 45, I49, I55-I57, I64, I67-I7I, I73-I73

télévision I2, III, I62, I87

terminologie 119

variation diachronique $104,114,162$

variation diaphasique 3-4, 156, 159, I 63 n.7, I64, 173

variation diastratique $4,63,104,156$, I69, 174

variation diatopique 3, 63, 104, II4, II7-II8, I59, I6I-3, I66-I67, 169, 173

vocabulaire 4, IOI, IO4, III, II5, II7, II9-I2O, 25I, 253 


\section{Modern French Identities}

Edited by Peter Collier

This series aims to publish monographs, editions or collections of papers based on recent research into modern French Literature. It welcomes contributions from academics, researchers and writers in British and Irish universities in particular.

Modern French Identities focuses on the French and Francophone writing of the twentieth century, whose formal experiments and revisions of genre have combined to create an entirely new set of literary forms, from the thematic autobiographies of Michel Leiris and Bernard Noël to the magic realism of French Caribbean writers.

The idea that identities are constructed rather than found, and that the self is an area to explore rather than a given pretext, runs through much of modern French literature, from Proust, Gide and Apollinaire to Kristeva, Barthes, Duras, Germain and Roubaud.

This series reflects a concern to explore the turn-of-thecentury turmoil in ideas and values that is expressed in the works of theorists like Lacan, Irigaray and Bourdieu and to follow through the impact of current ideologies such as feminism and postmodernism on the literary and cultural interpretation and presentation of the self, whether in terms of psychoanalytic theory, gender, autobiography, cinema, fiction and poetry, or in newer forms like performance art.

The series publishes studies of individual authors and artists, comparative studies, and interdisciplinary projects, including those where art and cinema intersect with literature.

Volume 1 Victoria Best \& Peter Collier (eds): Powerful Bodies.

Performance in French Cultural Studies.

220 pages. 1999. ISBN 3-906762-56-4 / US-ISBN 0-8204-4239-9

Volume 2 Julia Waters: Intersexual Rivalry.

A 'Reading in Pairs' of Marguerite Duras and Alain Robbe-Grillet. 228 pages. 2000. ISBN 3-906763-74-9 / US-ISBN 0-8204-4626-2 
Volume 3 Sarah Cooper: Relating to Queer Theory.

Rereading Sexual Self-Definition with Irigaray, Kristeva, Wittig and Cixous.

231 pages. 2000. ISBN 3-906764-46-X / US-ISBN 0-8204-4636-X

Volume 4 Julia Prest \& Hannah Thompson (eds): Corporeal Practices.

(Re)figuring the Body in French Studies.

166 pages. 2000. ISBN 3-906764-53-2 / US-ISBN 0-8204-4639-4

Volume 5 Victoria Best: Critical Subjectivities.

Identity and Narrative in the Work

of Colette and Marguerite Duras.

243 pages. 2000. ISBN 3-906763-89-7 / US-ISBN 0-8204-4631-9

Volume 6 David Houston Jones: The Body Abject: Self and Text in Jean Genet and Samuel Beckett.

213 pages. 2000. ISBN 3-906765-07-5 / US-ISBN 0-8204-5058-8

Volume 7 Robin MacKenzie: The Unconscious in Proust's A la recherche du temps perdu.

270 pages. 2000. ISBN 3-906758-38-9 / US-ISBN 0-8204-5070-7

Volume 8 Rosemary Chapman: Siting the Quebec Novel.

The Representation of Space in Francophone Writing in Quebec.

282 pages. 2000. ISBN 3-906758-85-0 / US-ISBN 0-8204-5090-1

Volume 9 Gill Rye: Reading for Change.

Interactions between Text Identity in Contemporary French

Women's Writing (Baroche, Cixous, Constant).

223 pages. 2001. ISBN 3-906765-97-0 / US-ISBN 0-8204-5315-3

Volume 10 Jonathan Paul Murphy: Proust's Art.

Painting, Sculpture and Writing in A la recherche du temps perdu. 248 pages. 2001. ISBN 3-906766-17-9 / US-ISBN 0-8204-5319-6

Volume 11 Julia Dobson: Hélène Cixous and the Theatre.

The Scene of Writing.

166 pages. 2002. ISBN 3-906766-20-9 / US-ISBN 0-8204-5322-6

Volume 12 Emily Butterworth \& Kathryn Robson (eds): Shifting Borders.

Theory and Identity in French Literature.

VIII + 208 pages. 2001.

ISBN 3-906766-86-1 / US-ISBN 0-8204-5602-0

Volume 13 Victoria Korzeniowska: The Heroine as Social Redeemer in the Plays of Jean Giraudoux.

144 pages. 2001. ISBN 3-906766-92-6 / US-ISBN 0-8204-5608-X 
Volume 14 Kay Chadwick: Alphonse de Châteaubriant:

Catholic Collaborator.

327 pages. 2002. ISBN 3-906766-94-2 / US-ISBN 0-8204-5610-1

Volume 15 Nina Bastin: Queneau’s Fictional Worlds.

291 pages. 2002. ISBN 3-906768-32-5 / US-ISBN 0-8204-5620-9

Volume 16 Sarah Fishwick: The Body in the Work of Simone de Beauvoir. 284 pages. 2002. ISBN 3-906768-33-3 / US-ISBN 0-8204-5621-7

Volume 17 Simon Kemp \& Libby Saxton (eds): Seeing Things. Vision, Perception and Interpretation in French Studies. 287 pages. 2002. ISBN 3-906768-46-5 / US-ISBN 0-8204-5858-9

Volume 18 Kamal Salhi (ed.): French in and out of France.

Language Policies, Intercultural Antagonisms and Dialogue. 487 pages. 2002. ISBN 3-906768-47-3 / US-ISBN 0-8204-5859-7

Volume 19 Genevieve Shepherd: Simone de Beauvoir's Fiction.

A Psychoanalytic Rereading. 262 pages. 2003. ISBN 3-906768-55-4 / US-ISBN 0-8204-5867-8

Volume 20 Lucille Cairns (ed.): Gay and Lesbian Cultures in France. 290 pages. 2002. ISBN 3-906769-66-6 / US-ISBN 0-8204-5903-8

Volume 21 Wendy Goolcharan-Kumeta: My Mother, My Country. Reconstructing the Female Self in Guadeloupean Women's Writing. 236 pages. 2003. ISBN 3-906769-76-3 / US-ISBN 0-8204-5913-5

Volume 22 Patricia O’Flaherty: Henry de Montherlant (1895-1972). A Philosophy of Failure. 256 pages. 2003. ISBN 3-03910-013-0 / US-ISBN 0-8204-6282-9

Volume 23 Katherine Ashley (ed.): Prix Goncourt, 1903-2003: essais critiques. 205 pages. 2004. ISBN 3-03910-018-1 / US-ISBN 0-8204-6287-X

Volume 24 Julia Horn \& Lynsey Russell-Watts (eds): Possessions. Essays in French Literature, Cinema and Theory. 223 pages. 2003. ISBN 3-03910-005-X / US-ISBN 0-8204-5924-0

Volume 25 Steve Wharton: Screening Reality. French Documentary Film during the German Occupation. 252 pages. 2006. ISBN 3-03910-066-1 / US-ISBN 0-8204-6882-7

Volume 26 Frédéric Royall (ed.): Contemporary French Cultures and Societies. 421 pages. 2004. ISBN 3-03910-074-2 / US-ISBN 0-8204-6890-8

Volume 27 Tom Genrich: Authentic Fictions.

Cosmopolitan Writing of the Troisième République, 1908-1940. 288 pages. 2004. ISBN 3-03910-285-0 / US-ISBN 0-8204-7212-3 
Volume 28 Maeve Conrick \& Vera Regan: French in Canada.

Language Issues.

186 pages. 2007. ISBN 978-3-03-910142-9

Volume 29 Kathryn Banks \& Joseph Harris (eds): Exposure.

Revealing Bodies, Unveiling Representations.

194 pages. 2004. ISBN 3-03910-163-3 / US-ISBN 0-8204-6973-4

Volume 30 Emma Gilby \& Katja Haustein (eds): Space.

New Dimensions in French Studies.

169 pages. 2005. ISBN 3-03910-178-1 / US-ISBN 0-8204-6988-2

Volume 31 Rachel Killick (ed.): Uncertain Relations.

Some Configurations of the 'Third Space' in Francophone Writings of the Americas and of Europe.

258 pages. 2005. ISBN 3-03910-189-7 / US-ISBN 0-8204-6999-8

Volume 32 Sarah F. Donachie \& Kim Harrison (eds): Love and Sexuality.

New Approaches in French Studies.

194 pages. 2005. ISBN 3-03910-249-4 / US-ISBN 0-8204-7178-X

Volume 33 Michaël Abecassis: The Representation of Parisian Speech in the Cinema of the 1930s.

409 pages. 2005. ISBN 3-03910-260-5 / US-ISBN 0-8204-7189-5

Volume 34 Benedict O’Donohoe: Sartre's Theatre: Acts for Life. 301 pages. 2005. ISBN 3-03910-250-X / US-ISBN 0-8204-7207-7

Volume 35 Moya Longstaffe: The Fiction of Albert Camus. A Complex Simplicity. 300 pages. 2007. ISBN 3-03910-304-0 / US-ISBN 0-8204-7229-8

Volume 36 Arnaud Beaujeu: Matière et lumière dans le théâtre de Samuel Beckett: Autour des notions de trivialité, de spiritualité et d'" autre-là ". 377 pages. 2010. ISBN 978-3-0343-0206-8

Volume 37 Shirley Ann Jordan: Contemporary French Women’s Writing: Women's Visions, Women's Voices, Women's Lives. 308 pages. 2005. ISBN 3-03910-315-6 / US-ISBN 0-8204-7240-9

Volume 38 Neil Foxlee: Albert Camus’s 'The New Mediterranean Culture’: A Text and its Contexts.

349 pages. 2010. ISBN 978-3-0343-0207-4

Volume 39 Michael O’Dwyer \& Michèle Raclot: Le Journal de Julien Green: Miroir d'une âme, miroir d'un siècle. 289 pages. 2005. ISBN 3-03910-319-9

Volume 40 Thomas Baldwin: The Material Object in the Work of Marcel Proust. 188 pages. 2005. ISBN 3-03910-323-7 / US-ISBN 0-8204-7247-6 
Volume 41 Charles Forsdick \& Andrew Stafford (eds): The Modern Essay in French: Genre, Sociology, Performance.

296 pages. 2005. ISBN 3-03910-514-0 / US-ISBN 0-8204-7520-3

Volume 42 Peter Dunwoodie: Francophone Writing in Transition.

Algeria 1900-1945.

339 pages. 2005. ISBN 3-03910-294-X / US-ISBN 0-8204-7220-4

Volume 43 Emma Webb (ed.): Marie Cardinal: New Perspectives.

260 pages. 2006. ISBN 3-03910-544-2 / US-ISBN 0-8204-7547-5

Volume 44 Jérôme Game (ed.): Porous Boundaries: Texts and Images in

Twentieth-Century French Culture.

164 pages. 2007. ISBN 978-3-03910-568-7

Volume 45 David Gascoigne: The Games of Fiction: Georges Perec and Modern French Ludic Narrative.

327 pages. 2006. ISBN 3-03910-697-X / US-ISBN 0-8204-7962-4

Volume 46 Derek O’Regan: Postcolonial Echoes and Evocations:

The Intertextual Appeal of Maryse Condé.

329 pages. 2006. ISBN 3-03910-578-7

Volume 47 Jennifer Hatte: La langue secrète de Jean Cocteau: la mythologie personnelle du poète et l'histoire cachée des Enfants terribles.

332 pages. 2007. ISBN 978-3-03910-707-0

Volume 48 Loraine Day: Writing Shame and Desire: The Work of Annie Ernaux. 315 pages. 2007. ISBN 978-3-03910-275-4

Volume 49 John Flower (éd.): François Mauriac, journaliste: les vingt premières années, 1905-1925.

352 pages. 2011. ISBN 978-3-0343-0265-4

Volume 50 Miriam Heywood: Modernist Visions: Marcel Proust's A la recherche du temps perdu and Jean-Luc Godard's Histoire(s) du cinéma.

277 pages. 2012. ISBN 978-3-0343-0296-8

Volume 51 Isabelle McNeill \& Bradley Stephens (eds): Transmissions:

Essays in French Literature, Thought and Cinema.

221 pages. 2007. ISBN 978-3-03910-734-6

Volume 52 Marie-Christine Lala: Georges Bataille, Poète du réel.

178 pages. 2010. ISBN 978-3-03910-738-4

Volume 53 Patrick Crowley: Pierre Michon: The Afterlife of Names.

242 pages. 2007. ISBN 978-3-03910-744-5

Volume 54 Nicole Thatcher \& Ethel Tolansky (eds): Six Authors in Captivity. Literary Responses to the Occupation of France during World War II. 205 pages. 2006. ISBN 3-03910-520-5 / US-ISBN 0-8204-7526-2 
Volume 55 Catherine Dousteyssier-Khoze \& Floriane Place-Verghnes (eds):

Poétiques de la parodie et du pastiche de 1850 à nos jours.

361 pages. 2006. ISBN 3-03910-743-7

Volume 56 Thanh-Vân Ton-That: Proust avant la Recherche: jeunesse et genèse d'une écriture au tournant du siècle.

285 pages. 2012. ISBN 978-3-0343-0277-7

Volume 57 Helen Vassallo: Jeanne Hyvrard, Wounded Witness:

The Body Politic and the Illness Narrative.

243 pages. 2007. ISBN 978-3-03911-017-9

Volume 58 Marie-Claire Barnet, Eric Robertson and Nigel Saint (eds):

Robert Desnos. Surrealism in the Twenty-First Century.

390 pages. 2006. ISBN 3-03911-019-5

Volume 59 Michael O’Dwyer (ed.): Julien Green, Diariste et Essayiste. 259 pages. 2007. ISBN 978-3-03911-016-2

Volume 60 Kate Marsh: Fictions of 1947: Representations of Indian Decolonization 1919-1962.

238 pages. 2007. ISBN 978-3-03911-033-9

Volume 61 Lucy Bolton, Gerri Kimber, Ann Lewis and Michael Seabrook (eds): Framed!: Essays in French Studies.

235 pages. 2007. ISBN 978-3-03911-043-8

Volume 62 Lorna Milne and Mary Orr (eds): Narratives of French Modernity:

Themes, Forms and Metamorphoses. Essays in Honour of David

Gascoigne.

365 pages. 2011. ISBN 978-3-03911-051-3

Volume 63 Ann Kennedy Smith: Painted Poetry: Colour in Baudelaire's Art Criticism.

253 pages. 2011. ISBN 978-3-03911-094-0

Volume 64 Sam Coombes: The Early Sartre and Marxism.

330 pages. 2008. ISBN 978-3-03911-115-2

Volume 65 Claire Lozier: De l'abject et du sublime: Georges Bataille, Jean Genet, Samuel Beckett.

327 pages. 2012. ISBN 978-3-0343-0724-6

Volume 66 Charles Forsdick and Andy Stafford (eds): La Revue: The TwentiethCentury Periodical in French.

379 pages. 2013. ISBN 978-3-03910-947-0

Volume 67 Alison S. Fell (ed.): French and francophone women facing war / Les femmes face à la guerre.

301 pages. 2009. ISBN 978-3-03911-332-3 
Volume 68 Elizabeth Lindley and Laura McMahon (eds):

Rhythms: Essays in French Literature, Thought and Culture.

238 pages. 2008. ISBN 978-3-03911-349-1

Volume 69 Georgina Evans and Adam Kay (eds): Threat: Essays in French

Literature, Thought and Visual Culture.

248 pages. 2010. ISBN 978-3-03911-357-6

Volume 70 John McCann: Michel Houellebecq: Author of our Times.

229 pages. 2010. ISBN 978-3-03911-373-6

Volume 71 Jenny Murray: Remembering the (Post)Colonial Self:

Memory and Identity in the Novels of Assia Djebar.

258 pages. 2008. ISBN 978-3-03911-367-5

Volume 72 Susan Bainbrigge: Culture and Identity in Belgian Francophone Writing: Dialogue, Diversity and Displacement.

230 pages. 2009. ISBN 978-3-03911-382-8

Volume 73 Maggie Allison and Angela Kershaw (eds): Parcours de femmes:

Twenty Years of Women in French.

313 pages. 2011. ISBN 978-3-0343-0208-1

Volume 74 Jérôme Game: Poetic Becomings: Studies in Contemporary French Literature.

263 pages. 2011. ISBN 978-3-03911-401-6

Volume 75 Elodie Laügt: L’Orient du signe: Rêves et dérives chez Victor Segalen, Henri Michaux et Emile Cioran.

242 pages. 2008. ISBN 978-3-03911-402-3

Volume 76 Suzanne Dow: Madness in Twentieth-Century French Women's Writing: Leduc, Duras, Beauvoir, Cardinal, Hyvrard.

217 pages. 2009. ISBN 978-3-03911-540-2

Volume 77 Myriem El Maïzi: Marguerite Duras ou l'écriture du devenir.

228 pages. 2009. ISBN 978-3-03911-561-7

Volume 78 Claire Launchbury: Music, Poetry, Propaganda: Constructing French Cultural Soundscapes at the BBC during the Second World War. 223 pages. 2012. ISBN 978-3-0343-0239-5

Volume 79 Jenny Chamarette and Jennifer Higgins (eds): Guilt and Shame: Essays in French Literature, Thought and Visual Culture.

231 pages. 2010. ISBN 978-3-03911-563-1

Volume 80 Vera Regan and Caitríona Ní Chasaide (eds): Language Practices and Identity Construction by Multilingual Speakers of French L2: The Acquisition of Sociostylistic Variation. 189 pages. 2010. ISBN 978-3-03911-569-3 
Volume 81 Margaret-Anne Hutton (ed.): Redefining the Real: The Fantastic in Contemporary French and Francophone Women's Writing. 294 pages. 2009. ISBN 978-3-03911-567-9

Volume 82 Elise Hugueny-Léger: Annie Ernaux, une poétique de la transgression. 269 pages. 2009. ISBN 978-3-03911-833-5

Volume 83 Peter Collier, Anna Magdalena Elsner and Olga Smith (eds): Anamnesia: Private and Public Memory in Modern French Culture. 359 pages. 2009. ISBN 978-3-03911-846-5

Volume 84 Adam Watt (ed./éd.): Le Temps retrouvé Eighty Years After/80 ans après: Critical Essays/Essais critiques.

349 pages. 2009. ISBN 978-3-03911-843-4

Volume 85 Louise Hardwick (ed.): New Approaches to Crime in French Literature, Culture and Film. 237 pages. 2009. ISBN 978-3-03911-850-2

Volume 86 Emmanuel Godin and Natalya Vince (eds): France and the Mediterranean: International Relations, Culture and Politics. 372 pages. 2012. ISBN 978-3-0343-0228-9

Volume 87 Amaleena Damlé and Aurélie L’Hostis (eds): The Beautiful and the Monstrous: Essays in French Literature, Thought and Culture. 237 pages. 2010. ISBN 978-3-03911-900-4

Volume 88 Alistair Rolls (ed.): Mostly French: French (in) Detective Fiction. 212 pages. 2009. ISBN 978-3-03911-957-8

Volume 89 Bérénice Bonhomme: Claude Simon: une écriture en cinéma. 359 pages. 2010. ISBN 978-3-03911-983-7

Volume 90 Barbara Lebrun and Jill Lovecy (eds): Une et divisible? Plural Identities in Modern France.

258 pages. 2010. ISBN 978-3-0343-0123-7

Volume 91 Pierre-Alexis Mével \& Helen Tattam (eds): Language and its Contexts/ Le Langage et ses contextes: Transposition and Transformation of Meaning?/Transposition et transformation du sens ? 272 pages. 2010. ISBN 978-3-0343-0128-2

Volume 92 Alistair Rolls and Marie-Laure Vuaille-Barcan (eds): Masking Strategies: Unwrapping the French Paratext. 202 pages. 2011. ISBN 978-3-0343-0746-8

Volume 93 Michaël Abecassis et Gudrun Ledegen (éds): Les Voix des Français Volume 1: à travers l'histoire, l'école et la presse. 372 pages. 2010. ISBN 978-3-0343-0170-1 
Volume 94 Michaël Abecassis et Gudrun Ledegen (éds): Les Voix des Français Volume 2: en parlant, en écrivant. 481 pages. 2010. ISBN 978-3-0343-0171-8

Volume 95 Manon Mathias, Maria O’Sullivan and Ruth Vorstman (eds): Display and Disguise.

237 pages. 2011. ISBN 978-3-0343-0177-0

Volume 96 Charlotte Baker: Enduring Negativity: Representations of Albinism in the Novels of Didier Destremau, Patrick Grainville and Williams Sassine. 226 pages. 2011. ISBN 978-3-0343-0179-4

Volume 97 Florian Grandena and Cristina Johnston (eds): New Queer Images: Representations of Homosexualities in Contemporary Francophone Visual Cultures. 246 pages. 2011. ISBN 978-3-0343-0182-4

Volume 98 Florian Grandena and Cristina Johnston (eds): Cinematic Queerness: Gay and Lesbian Hypervisibility in Contemporary Francophone Feature Films. 354 pages. 2011. ISBN 978-3-0343-0183-1

Volume 99 Neil Archer and Andreea Weisl-Shaw (eds): Adaptation: Studies in French and Francophone Culture. 234 pages. 2012. ISBN 978-3-0343-0222-7

Volume 100 Peter Collier et Ilda Tomas (éds): Béatrice Bonhomme: le mot, la mort, l'amour.

437 pages. 2013. ISBN 978-3-0343-0780-2

Volume 101 Helena Chadderton: Marie Darrieussecq’s Textual Worlds: Self, Society, Language.

170 pages. 2012. ISBN 978-3-0343-0766-6

Volume 102 Manuel Bragança: La crise allemande du roman français, 1945-1949: la représentation des Allemands dans les best-sellers de l'immédiat après-guerre.

220 pages. 2012. ISBN 978-3-0343-0835-9

Volume 103 Bronwen Martin: The Fiction of J.M.G. Le Clézio: A Postcolonial Reading.

199 pages. 2012. ISBN 978-3-0343-0162-6

Volume 104 Hugues Azérad, Michael G. Kelly, Nina Parish et Emma Wagstaff (éds): Chantiers du poème: prémisses et pratiques de la création poétique moderne et contemporaine.

374 pages. 2013. ISBN 978-3-0343-0800-7

Volume 105 Franck Dalmas: Lectures phénoménologiques en littérature française: de Gustave Flaubert à Malika Mokeddem. 253 pages. 2012. ISBN 978-3-0343-0727-7 
Volume 106 Béatrice Bonhomme, Aude Préta-de Beaufort et Jacques Moulin (éds): Dans le feuilletage de la terre: sur l'œuvre poétique de Marie-Claire Bancquart.

533 pages. 2013. ISBN 978-3-0343-0721-5

Volume 107 Claire Bisdorff et Marie-Christine Clemente (éds): Le Cœur dans tous ses états: essais sur la littérature et l'art français.

230 pages. 2013. ISBN 978-3-0343-0711-6

Volume 108 Michaël Abecassis et Gudrun Ledegen (éds): Écarts et apports des médias francophones: lexique et grammaire.

300 pages. 2013. ISBN 978-3-0343-0882-3

Volume 109 Maggie Allison and Imogen Long (eds): Women Matter / Femmes Matière: French and Francophone Women and the Material World. 273 pages. 2013. ISBN 978-3-0343-0788-8

Volume 110 Fabien Arribert-Narce et Alain Ausoni (éds): L'Autobiographie entre autres: écrire la vie aujourd'hui.

221 pages. 2013. ISBN 978-3-0343-0858-8

Volume 111 Leona Archer and Alex Stuart (eds): Visions of Apocalypse:

Representations of the End in French Literature and Culture.

266 pages. 2013. ISBN 978-3-0343-0921-9

Volume 112 Simona Cutcan: Subversion ou conformisme? La différence des sexes dans l'œuvre d'Agota Kristof.

264 pages. 2014. ISBN 978-3-0343-1713-9

Volume 113 Owen Heathcote: From Bad Boys to New Men? Masculinity, Sexuality and Violence in the Work of Éric Jourdan.

279 pages. 2014. ISBN 978-3-0343-0736-9

Volume 114 Ilda Tomas: Arc-en-ciel: études sur divers poètes.

234 pages. 2014. ISBN 978-3-0343-0975-2

Volume 115 Lisa Jeschke and Adrian May (eds): Matters of Time: Material Temporalities in Twentieth-Century French Culture.

314 pages. 2014. ISBN 978-3-0343-1796-2

Volume 116 Crispin T. Lee: Haptic Experience in the Writings of Georges Bataille, Maurice Blanchot and Michel Serres.

316 pages. 2014. ISBN 978-3-0343-1791-7

Volume 117 Ashwiny O. Kistnareddy: Locating Hybridity: Creole, Identities and Body Politics in the Novels of Ananda Devi.

208 pages. 2015. ISBN 978-3-0343-1814-3

Volume 118 Michaël Abecassis et Gudrun Ledegen (éds): De la genèse de la langue à Internet: variations dans les formes, les modalités et les langues en contact.

278 pages. 2015. ISBN 978-3-0343-1798-6 